•研究报告・

\title{
全国陆生野生动物调查单元区划方案
}

\author{
郜二虎 ${ }^{*}$ 何杰坤 ${ }^{2}$ 王志臣 $^{1}$ 徐 扬 ${ }^{2}$ 唐小平 ${ }^{1} \quad$ 江海声 $^{2}$ \\ 1 (国家林业局调查规划设计院, 北京 100714) \\ 2 (华南师范大学生命科学学院, 广州 510631)
}

\begin{abstract}
摘要: 我国早期的动物地理区划主要依靠专家知识和经验, 缺乏系统的定量分析, 而且部分基本区划单元内的动 物生态成分差异仍然较大, 使其在野生动物保护管理应用中受到一定限制。为满足野生动物保护管理的需要, 尤 其为满足全国第二次陆生野生动物资源调查的需要，我们在张荣祖“中国动物地理区划”的基础上，进行了全国野 生动物调查单元区划。将全国陆地区域划分为 $5 \mathrm{~km} \times 5 \mathrm{~km}$ 的网格, 基于 1 ,784种陆栖脊椎动物(262种两栖动物、358 种爬行动物、814种鸟类、350种兽类)的分布数据，对各网格内的动物分布型比例及自然环境因子进行聚类分析。 根据聚类结果, 并结合指示物种的分布情况以及省级行政区划界线, 将全国划分为 2 界 7 区 19 亚区 54 个动物地理省 239 个生态地理单元 310 个调查单元。与张荣祖“中国动物地理区划”方案相比, 界、区、亚区、动物地理省的数量 一致，边界走向基本一致，但也有一些界线不同，而且增加了“生态地理单元”和“调查单元” 2级区划单元。

关键词：野生动物; 区划; 聚类分析; 动物地理; 生态地理单元; 调查单元
\end{abstract}

\section{China's zoogeographical regionalization based on terrestrial vertebrates}

Erhu $\mathrm{Gao}^{1 *}$, Jiekun $\mathrm{He}^{2}$, Zhichen Wang ${ }^{1}$, Yang $\mathrm{Xu}^{2}$, Xiaoping Tang ${ }^{1}$, Haisheng Jiang ${ }^{2}$

1 Academy of Forest Inventory and Planning, State Forestry Administration, Beijing 100714

2 School of Life Sciences, South China Normal University, Guangzhou 510631

\begin{abstract}
China's previous zoogeographical regionalization designations were delineated according to expert knowledge and experience. However, these previous designations have limited application in wildlife conservation and management because of a lack of quantitative footing. Additionally, there are still significant differences in the ecological components of some of the basic units. In order to meet the needs of wildlife protection and management, especially the needs of the Second National Survey on Terrestrial Wildlife Resources in China, we carried out national wildlife survey unit zoning, based on Zhang Rongzu's "Zoogeographical Regions of China". In this study, hierarchical clustering was applied to a $5 \mathrm{~km} \times 5 \mathrm{~km}$ grid of faunistic and environmental components, based on a comprehensive species distribution dataset, consisting of 262 amphibians, 358 reptiles, 814 birds, and 350 mammals. Our results delineated China into 2 realms, 7 regions, 19 sub-regions, 54 zoogeographical provinces, 239 eco-geographical units, and 310 survey units. Compared to Zhang's scheme, the number of realms, regions, sub-regions, and zoogeographical provinces are the same and most of the boundaries are broadly consistent, however, some discrepancies also emerge. To meet the needs of wildlife conservation and management, two hierarchical levels of zoogeographical units were added, namely eco-geographical units and survey units.
\end{abstract}

Key words: wildlife; regionalization; clustering; zoogeography; eco-geographical unit; survey unit

动物地理区划表明了动物分布的区域差异(张 荣祖, 2011), 反映了各地动物对地质历史过程及现 今生态环境适应的结果。它不仅为历史生物地理 学、生态生物地理学、进化生物学和系统分类学的
许多问题提供了清晰的空间框架 $(K r e f t \&$ Jetz, 2010), 而且在保护生物学方面得到了广泛应用(De Klerk et al, 2002; Kreft \& Jetz, 2010; Holt et al, 2013)。 
早在 20 世纪 50 年代, 我国就开始了动物地理 区划工作。寿振黄(1955)将我国毛皮兽的分布划分 为 8 个区; 郑作新和张荣祖(1959)将我国陆地区域 划分为 7 区 16 亚区, 后经张荣祖多次修改, 将“中 国动物地理区划”修订为 2 界 7 区 19 亚区 54 动物 地理省(张荣祖, 2011)。该区划方案得到广泛认可, 目前仍是我国野生动物方面最重要的全国区划。但 是, 由于受历史条件和技术手段的限制, 我国早期 的动物地理区划主要依靠专家知识和经验, 缺乏系 统的定量分析。随着统计模型的发展和地理信息系 统的应用, 我国学者逐步探索采用定量研究方法进 行动物地理区划或生物地理区划(李晓晨, 2000; 解 炎等, 2002; Xiang et al, 2004; He et al, 2017)。例如, 解炎等根据 171 种兽类和 509 种植物的分布信息, 利用 GIS 技术和定量方法把全国划分为 4 个区域(8 个亚区域)、27 个生物地理区和 124 个生物地理单 元(解炎, 2000; 解炎等, 2002; Xie et al, 2004)。He 等 (2017)根据 2,102 种陆栖冷椎动物的分布信息, 将全 国划分为 10 个动物地理区。但是, 由于各类区划的 研究目的、类群、尺度及方法的差异, 不同区划结 果的差异较大, 而且在这些区划中, 部分基本区划 单元内动物生态成分差异仍然较大, 在全国野生 动物资源调查及保护管理实践中受到一定程度的 限制。

因此, 为满足现阶段我国野生动物保护管理需 要, 尤其为满足全国第二次陆生野生动物资源调查 技术方案(郜二虎等, 2014)的要求, 我们在张荣祖 (2011)“中国动物地理区划”的基础上，根据 1,784 种 兽类、鸟类、爬行类和两栖类动物的分布信息及自 然环境数据, 进行了全国动物地理区划, 划定了全 国陆生野生动物调查单元, 以便为我国野生动物资 源调查和保护管理提供参考。

\section{1 材料与方法}

\section{1 物种分布数据}

根据我国两栖动物(费梁等, 2006, 2009a, b)、爬 行动物(张孟闻等, 1998; 赵尔宓等, 1998, 1999)、鸟 类(郑光美, 2011)和兽类(王应祥, 2003)名录进行统 计, 我国已记录到陆栖冷椎动物 4 纲 41 目 178 科 2,668 种。我们根据《中国动物志》、各省动物志 以及其他公开发表的文献(附录 1)中关于物种分布 的记录, 以县(指县级行政区划)为单位, 建立了全
国物种分布数据库。由于兽类中的翼手目物种和迁 徙鸟类不适合用于陆栖脊椎动物的区划(Wallace, 1876; Rueda et al, 2013), 因此进行区划时, 我们剔 除了兽类中的翼手目物种 (120 种)以及不在我国繁 殖的迁徙鸟类(156 种)。此外, 分布型(张荣祖, 2011) 不明确的物种(240 种)和分布资料不详的物种 (368 种)也不用于本区划(附录 2)。最终, 实际用于本区 划的物种数为 1,784 种, 包括 262 种两栖动物、358 种爬行动物、814 种鸟类和 350 种兽类(附录 3), 约 占我国陆栖脊椎动物种数的 $66.9 \%$ 。

\section{2 物种适生栖息地模型}

由于我国各县面积差异较大, 以各县记录作为 物种的分布区域将会夸大某些物种的分布范围。因 此，我们采用物种适生栖息地模型(Rondinini et al, 2011), 将物种的县域记录转换为面积相等的 $5 \mathrm{~km} \times$ $5 \mathrm{~km}$ 网格记录。首先, 根据动物志及相关文献(附录 1), 整理形成了各物种的适生栖息地信息库, 主要 包括物种适宜栖息的植被类型和海拔区间。然后, 将全国陆地划分为面积相等的 $5 \mathrm{~km} \times 5 \mathrm{~km}$ 网格, 提取每个网格所处的县级行政区划名称、植被类型 和海拔区间等基础信息。就各县有记录的每一物种, 将各个网格的基础信息(植被类型、海拔)与每个物 种的适生栖息地信息(植被类型、海拔)进行对比，确 定各个网格的物种组成(附录 4)。

\section{3 分布型}

分布型是指具有相似空间分布的物种组群, 指 物种在全球范围内的分布, 而不是在局部区域的分 布。分布型反映了物种分布对历史压力和环境压力 的响应结果 (Passalacqua，2015; Fattorini，2015, 2016), 其形成是动物适应环境历史变迁直至现阶 段的结果, 也是动物在分布上趋同演化的一组事件 (张荣祖, 2011)。物种分布型的相似程度体现了不同 地域的区系特征(张有瑜等, 2008), 被广泛运用于动 物和植物的地理区划中(例如: Xing et al, 2008; 张 有瑜等, 2008; 冯建孟和朱有勇, 2010; 田怀珍等, 2013)。根据我国已有的陆栖脊椎动物分布型分类系 统(张荣祖, 2011), 我们整理了 1,784 个物种的分布 型信息(附录 5,6), 并根据各区域物种分布型比例 对高级单元(界、区、亚区)进行了地理区划。

\section{4 环境指标}

采用温度、降水、地形、地貌、植被和土壤等 环境因子作为亚区以下单元(即地理省和生态地理 
单元)的区划指标(附录 7)。其中温度和降水数据来

源于 WorldClim 数据集(http://www.worldclim.org, Hijmans et al, 2005), 数字高程数据 $(90 \mathrm{~m} \times 90 \mathrm{~m})$ 来 源于国际农业研究磋商组织空间信息协会 (the Consortium for Spatial Information of the Consultative Group for International Agricultural Research) (http://www.cgiar-csi.org/data/srtm-90m-digital-elevat ion-database-v4-1), 数字化的地貌图 (1：400 万)和 土壤图(1：100 万)来源于国家地球系统科学数据共 享平台(http://www.geodata.cn/), 植被图来源于《中 华人民共和国植被图(1：100 万)》(张新时, 2007)。

\section{5 区划方法}

首先, 以 $5 \mathrm{~km} \times 5 \mathrm{~km}$ 的网格为基本区划单位, 形成“网格号 $\times$ 动物分布型比例”的排列矩阵。然 后, 采用欧氏距离计算网格与网格间的距离矩阵, 并用 Ward 方法对其进行系统聚类分析。根据动物 地理区划“历史发展”、“生态适应”与“生产实践”的 三项基本原则(张荣祖, 2011), 为满足全国第二次陆 生野生动物资源调查和保护管理需要, 我们遵循现 有的动物地理区划系统(张荣祖, 2011), 按照动物区 系成分差异, 划分界、区和亚区; 在各个亚区内, 对 各网格的自然环境因子进行聚类分析, 根据聚类结 果, 并结合指示物种的分布情况, 划分动物地理省 和生态地理单元。最后, 若一个生态地理单元跨不 同的行政省, 则利用行政省边界进行切割, 划分成 不同的调查单元; 不跨行政省的生态地理单元则为 一个调查单元。

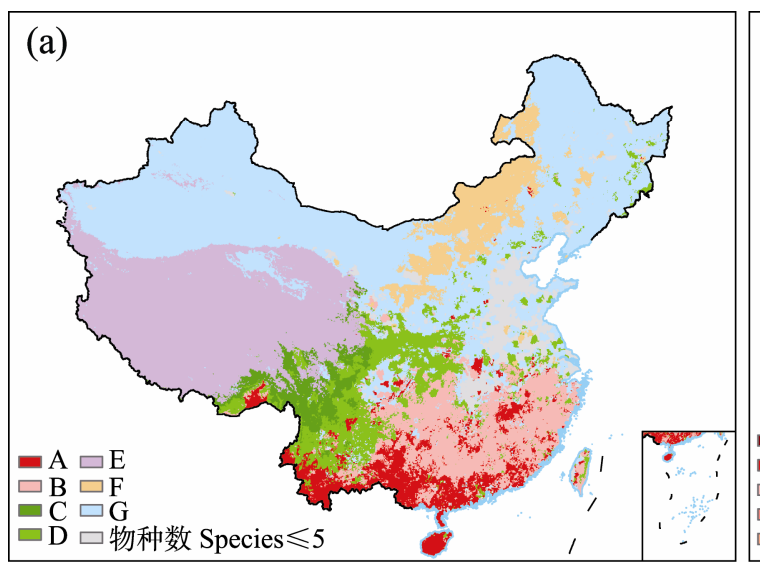

\section{2 结果}

\section{1 地理区划}

根据各网格动物分布型比例的系统聚类结果, 当聚类树划分为 7 类时, 全国可识别出 7 个区域(图 1a)。A 区包括我国热带和南亚热带地区; B 区包括 秦岭-淮河以南、南岭以北、横断山区以东的区域; $\mathrm{C}$ 区和 D 区包括云贵高原和横断山区; E 区包括青藏 高原; $\mathrm{F}$ 区包括内蒙古高原; G 区包括除内蒙古高原 以外的广大北方地区(图 1a)。当聚类树划分为 19 类时, 各小区也得到了较好的划分(图 1b)。例如, A 区被划分为云南南部 $(A a)$ 、两广和福建南部 $(A b)$ 及 台湾 $(\mathrm{Ab}+\mathrm{Bc}) 3$ 个小区; $\mathrm{E}$ 区被划分为青藏高原腹地 (Ea)和青海藏南地区(Eb) 2 个小区(图 1b)。

因此, 根据各网格动物分布型比例的系统聚类 结果, 华南区、华中区、西南区、青藏区能被很好 地识别出来, 东北区、华北区和蒙新区(除内蒙古高 原)的分界并不十分明显, 但从我国主要动物分布 型的分布格局(图 2)可以看出: 古北型物种广泛分 布于我国北方地区; 东北型物种分布于我国东北及 华北地区, 但以东北为主; 中亚型物种主要分布于 我国内蒙古和新疆一带。据此并结合张荣祖(2011) 的中国动物地理区划方案, 可以划分出东北区、华 北区和蒙新区。此外, 根据各网格动物环境指标聚 类结果, 我们在 19 个亚区以下进一步划分了 54 个 动物地理省 239 个生态地理单元 310 个调查单元。 最终, 我们将全国划分为 2 界 7 区 19 亚区 54 个动

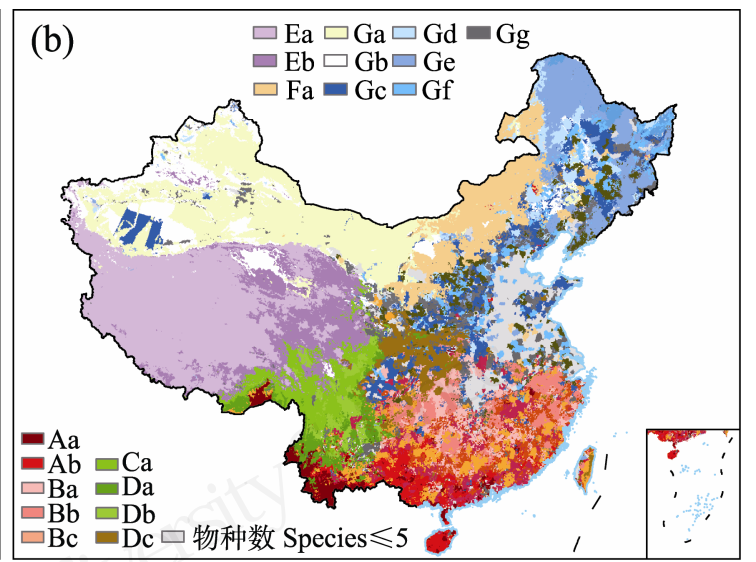

图 1 各网格动物分布型比例聚类结果示意图: (a) 7 类; (b) 19 类。其中 A-G 表示可识别出的 7 个不同区域; Aa、Ab 表示 A 区可进一步识别出 Aa、Ab 两个区域, 以此类推。

Fig. 1 Results from hierarchical clustering on chorotype proportion of each grids. (a) 7 clusters and (b) 19 clusters. Codes from A to $\mathrm{G}$ in the figures representing 7 zones that can be identified, while codes Aa and Ab representing 2 zones that can be further identified in zone $\mathrm{A}$, and so on. 

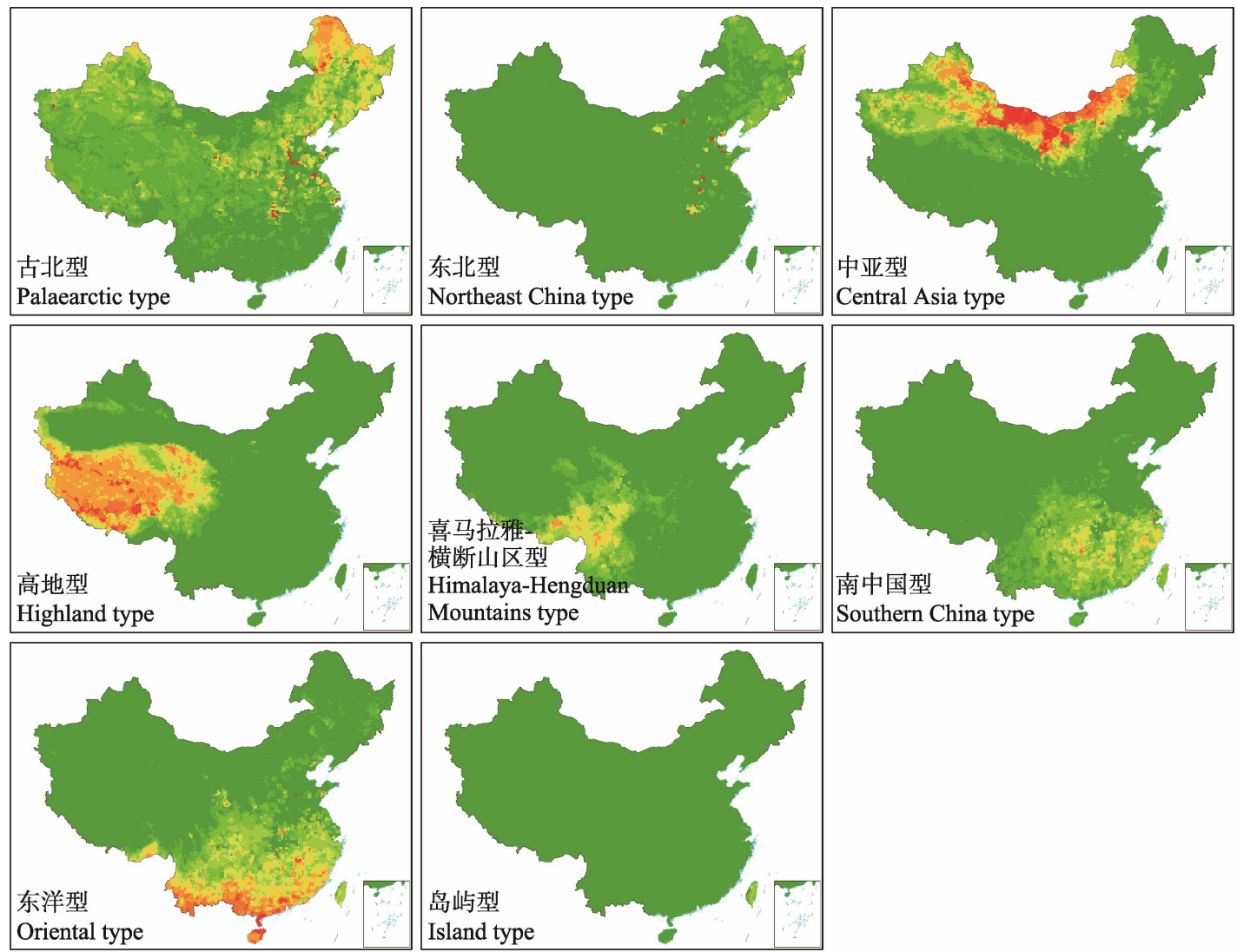

Southern China type

50

(\%)

图 2 我国主要动物分布型的分布格局

Fig. 2 Distribution patterns of the main chorotypes in China

物地理省 239 个生态地理单元 310 个调查单元(图 3 , 附录 8)。

\section{2 各地理区动物区系差异}

根据区划结果, 我们对 7 个区中的动物区系成 分进行了分析。结果显示: 古北界与东洋界的区系 差异主要是东洋型成分比例不同。在东洋界, 东洋 型成分大于 $16.7 \%$, 而在古北界, 东洋型成分小于 $16.7 \%$ (图 4)。

在古北界，青藏区以高地型成分占优(>29\%), 特别是在青藏高原腹地, 高地型成分所占比例超过 $50 \%$ 。而蒙新区则含有较高比例的中亚型成分 $(>15 \%)$, 并含有古北型和全北型成分, 这 3 类区系 成分在蒙新区各亚区间各有优势。其中，东部草原 亚区以中亚型占优( $>35 \%)$, 天山山地亚区则以古北 型为主 $(>27 \%)$ 。东北区和华北区的分异主要表现为 东北型成分所占比例不同。在东北区，东北型成分 大于 $7.5 \%$, 而在华北区，东北型成分则小于 $7.5 \%$ (图 4)。
在东洋界，西南区具有独特的动物区系(喜马 拉雅-横断山区型>19\%), 西南区的动物区系成分 既有北方成分的高地型，也有南方成分的南中国型 和东洋型，其自身也形成独特的喜马拉雅-横断山 区型。华中区和华南区的分异主要表现为东洋型成 分所占比例的不同(图 4), 在华中区，动物区系成分 较为简单, 以南中国型和东洋型为主, 东洋型成分 不超过 $56.5 \%$; 华南区则具有典型的热带性，东洋 型成分超过 $56.5 \%$ 。

\section{3 古北界与东洋界分界}

古北界和东洋界的划分一直以来存在争议。根 据聚类分析，我国古北界和东洋界的东段基本可 沿秦岭-淮河一线划分(图 1a), 在我国西部地区, 古北界和东洋界的分界线穿过横断山区，但具体 分界在水平方向上则难以确定。为此, 我们分析了 横断山区不同海拔区间各种动物分布型的比例, 结果显示，南方类型的分布型比例随海拔升高而逐 渐减少，北方类型的分布型比例随海拔升高而逐渐 


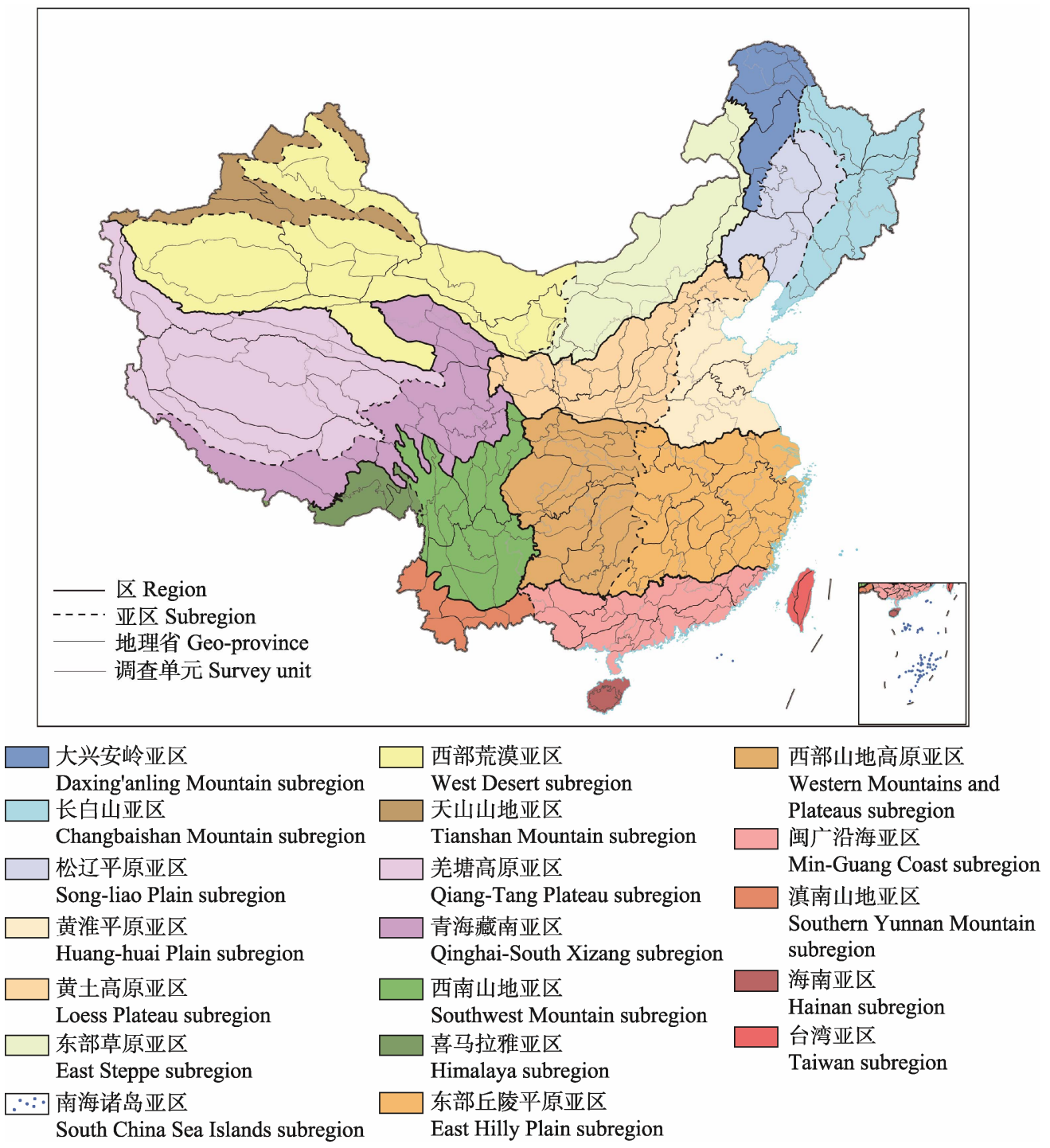

图 3 全国陆生野生动物调查单元区划图

Fig. 3 Map of zoogeographical regions of China for terrestrial wildlife survey

增多, 海拔 3,500 $\mathrm{m}$ 以下南方类型动物成分占优, 海拔 4,000 $\mathrm{m}$ 以上北方类型的动物成分占优，海拔 3,500-4,000 m 区间成为两大动物区系成分优势度 转换的分界(图 5)。因此, 本方案根据两大动物区系 成分优势度转换的分界, 将高原山脉向南延伸的区 域划入古北界，将河流山谷向北延伸的区域划入东 洋界。

\section{3 讨论}

总体上，根据各网格物种分布型比例进行聚类 分析, 能较好地识别出我国已有动物地理区划的 2 界 7 区和 19 个亚区。此外, 为满足我国野生动物调 查及管理需要, 我们在亚区以下进一步划分为 54
个地理省 239 个生态地理单元及 310 个调查单元。 我们的结果支持古北界与东洋界分界的东段位于秦 岭-淮河一线, 并表明其分界的西段大致在横断山 地区海拔 3,500-4,000 $\mathrm{m}$ 之间。

\section{1 动物地理区划}

本方案的区、亚区、地理省的数量与张荣祖 (2011)区划方案中的数量一致, 边界走向基本一致, 但一些界线有所差异。其中有 4 处界线差异较大:

(1)张荣祖(2011)方案中，东北区和蒙新区的分 界线大致在大兴安岭山脉主脊一白城-通辽-阜新一 线; 本方案东北区和蒙新区的分界线则大致沿大兴 安岭西部山麓-扎鲁特旗-巴林左旗-林西县一线, 即在阿尔山以南, 将二者的分界线向西移约 $200 \mathrm{~km}$, 


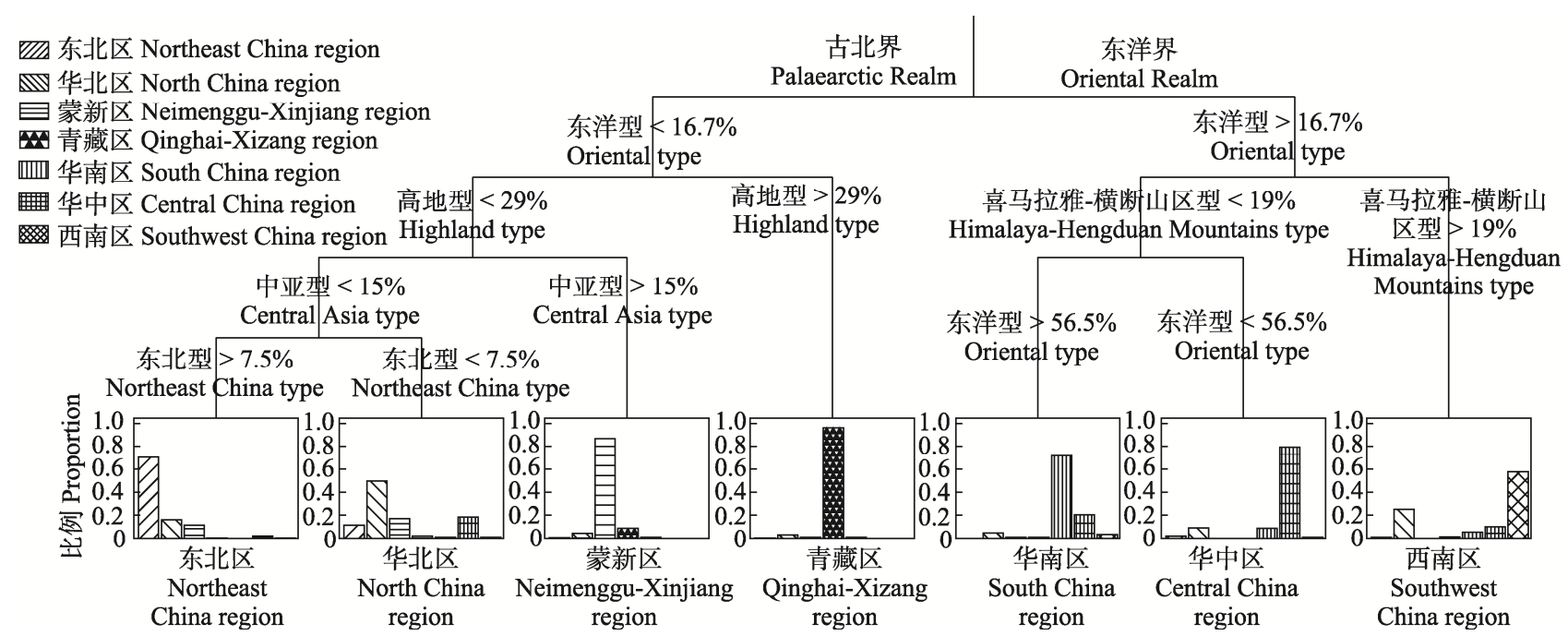

图 47 个地理区的动物区系成分分类树

Fig. 4 Classification tree on the regional fauna of seven zoogeographical regions

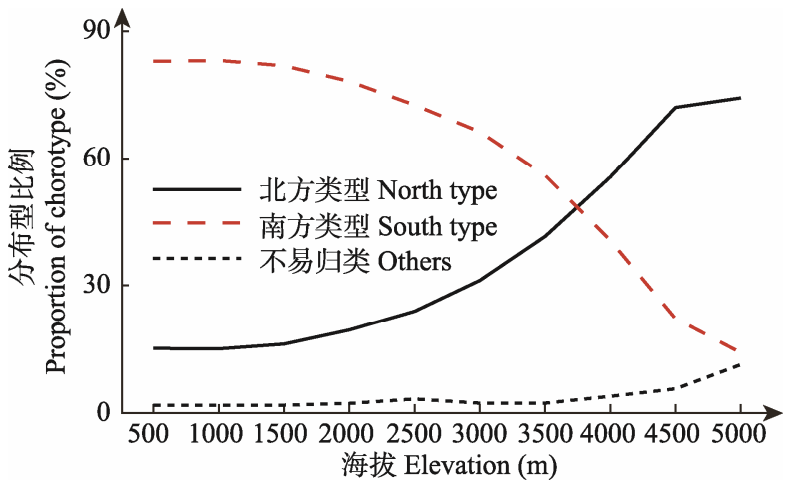

图 5 横断山区不同海拔区间各动物分布型比例

Fig. 5 Proportion of chorotype along elevation gradients in the Hengduanshan Mountains

大致移到了森林与草原的分界线, 将大兴安岭南部 山前台地、辽河平原和科尔沁沙地划到了东北区。 理由如下: (1)大兴安岭南部山前台地、辽河平原和 科尔沁沙地动物区系成分以古北型为主, 中亚型成 分不足 $20 \%$; 而蒙新区动物的中亚型成分可达 $40 \%$, 甚至在部分地区可达 $50 \%$ 以上。(2)大兴安岭南部山 前台地以农田类型的植被为主, 且分布少量温带落 叶阔叶林; 辽河平原和科尔沁沙地由于近代受到人 类干扰后出现土地退化, 植被呈现温带落叶润叶 林、温带草原和中温带农田、经济作物三者交错的 格局，而蒙新区的植被以温带典型草原为主。

(2)本区划西南区与华南区在云南的分界线为 从高黎贡山南端-无量山南端-哀牢山南端, 至蒙自丘北-金钟山一线, 比张荣祖(2011)方案界线偏南, 主要是将无量山和哀牢山划到了西南区。西南区和
华南区动物成分的差异主要是喜马拉雅-横断山区 型所占比例不同(图 4), 而偏移区域(无量山和哀牢 山)动物区系成分中, 喜马拉雅-横断山区型占到 $29.1 \%$ ，明显高于华南区，因此划为西南区。

(3)张荣祖(2011)方案中，蒙新区东部草原亚区 和西部荒漠亚区的分界约在二连浩特-银川一线; 而本方案东部草原亚区和西部荒漠亚区的分界约 在贺兰山-乌海-狼山一线，比前者平均西移约 100 $\mathrm{km}$ 。理由如下: 贺兰山一线是我国 $200 \mathrm{~mm}$ 等降水 量线，也是我国温带草原和荒漠的分界线; 典型荒 漠物种如新疆漠虎(Alsophylax przewalskii)、白尾地 鸦(Podoces biddolphi)、野双峰驼(Camelus ferus)只 分布于贺兰山一线以西，而典型草原物种如布氏田 鼠 (Lasiopodomys brandtii)、草原鼠鼠 (Myospalax aspalax)等主要分布于此线以东。

(4)张荣祖(2011)方案中, 㒸塘高原亚区与青海 藏南亚区的分界约在布尔汗布达山-玛多-玉树-那 曲-冈底斯山一线, 而本方案则约在布尔汗布达山扎陵湖-曲麻莱-巴青-冈底斯山一线。偏移区域主 要是通天河河谷地区, 该区域 $\geq 0{ }^{\circ} \mathrm{C}$ 的积温较高, 动物区系成分以高地型和喜马拉雅-横断山区型为 主，且二者比重均衡，更接近于青海藏南亚区的动 物区系成分, 因此划为青海藏南亚区。

此外, 已有的中国动物地理区划中 (张荣祖, 2011), 最小的区划单元为“地理省”, 其平均面积为 175,800 $\mathrm{km}^{2}$ (5\%: 13,347 $\mathrm{km}^{2}$; 95\%: 383,323 km²), 难以满足我国野生动物资源调查和保护管理的需 
要。解炎等所提出的我国生物地理区划方案共划分 了 124 个地理单元, 但是其区划选择的类群为 171 种兽类和 509 种植物(解炎, 2000; 解炎等, 2002; Xie et al, 2004), 且其区划系统与已被我国广泛接受的 区划系统差异较大，在生产实践中应用时容易产生 困惑。本研究在已有的 2 界 7 区 19 亚区 54 个地理 省的区划系统基础上, 增加了生态地理单元和调查 单元的划分, 既能较好地衔接已有的区划成果, 也 满足了我国野生动物保护实践的需要。

\section{2 古北界与东洋界分界}

按照现代陆栖脊椎动物的研究, 我国陆地动物 区系分属于古北界和东洋界两界(Wallace, 1876; Hoffmann, 2001; Kreft \& Jetz, 2010)。但关于两界的 分界线尚存在许多争议。其中关于古北界和东洋界 在我国东部地区的分界线有 19 种意见(Hoffmann, 2001; 张荣祖, 2011)。在这些意见中, 最北的界线超 过 $40^{\circ} \mathrm{N}$, 最南的可至 $20^{\circ} \mathrm{N}$, 两者之间的距离超过 $2,000 \mathrm{~km}$ (He et al, 2017)。张荣祖(2011)认为, 古北 界和东洋界的分界在我国东部地区为一广泛的过 渡地带, 并把秦岭一淮河一线作为其分界线。我们的 结果也表明: 在秦岭-淮河以南, 动物以东洋型、南 中国型区系成分为主; 在秦岭-淮河以北, 动物以 古北型和东北型区系成分为主。这个结果与张荣祖 (2011)区划方案一致。此外, 很多地理区划和生态区 划都以秦岭-淮河一线作为南北分界(罗开富, 1954; 黄秉维, 1958; 赵松乔, 1983; 任美锷, 1999; 郑度, 2008), 体现了此界限作为地理屏障的阻隔作用。

在我国西部地区, 古北界和东洋界的分界线穿 过横断山区, 但由于横断山区南北纵列的山脉一峡 谷地貌, 明显形成了两界动物区系相互交流的通 道。在海拔较高的地方, 有不少古北界的种类沿山 脊向南伸展, 而不少热带种类则沿河谷向北分布, 使该地区两界动物成分混杂。因此, 关于古北界和 东洋界在我国西部地区的分界, 也有一些不同意见 (Chen et al, 2008; 张荣祖, 2011)。张荣祖(2011)认为, 鉴于横断山区复杂的三维空间变化, 古北界和东洋 界的确切界线不易确定, 因此, 在其区划方案中暂 以虚线表示。我们的结果显示, 古北界和东洋界在 横断山区的分界虽然难以用水平方向上的界线表 达, 但在垂直方向上仍然可以找到其分界: 海拔 3,500-4,000 $\mathrm{m}$ 的区间是两大动物区系的分水岭。 这个结果与龚正达等(1999)对云南苍山虫类分布研
究(2,900-3,300 m), 孙治宇等(2007)对四川海子山 大中型兽类分布研究 (3,800-4,400 m), 以及涂飞云 等(2012)对四川夹金山小型兽类分布研究 $(3,600 \mathrm{~m})$ 的结论相似。

\section{3 不足}

由于动物分布数据的不足以及区划方法的局 限性, 采用定量化方法进行动物地理区划依然存在 许多问题(He et al, 2017)。第一, 由于缺少调查等原 因, 在物种分布数据库中, 部分地区的物种记录仍 然偏少, 这可能引起区划过程中的随机误差和系统 误差(Lennon et al, 2001; Kreft \& Jetz, 2010)。第二, 随着物种系统发育研究不断深入, 在区划中融入物 种系统发育信息成为可能(Holt et al, 2013), 但由于 不同类群间物种系统发育信息的精度和分化时间 的不足, 本区划尚未将其考虑在内。第三, 仅仅根 据物种分布型比例的聚类结果, 没有明显识别出东 北区、华北区和蒙新区(除内蒙古高原外), 可能是由 于古北型物种在我国北方地区分布广泛, 且数量较 多所致。第四, 由于我国地域广阔, 各地区动物组 成差异大, 尤其热带(亚热带)及山地地区的动物组 成差异大于温带及平原地区, 在统一系统聚类树里 划定若干个群集(生态地理单元)显得尤为困难。因 此, 在划分地理省及以下单元时, 我们采取在各个 亚区之下进行聚类的方法划定生态地理单元, 但 这可能造成各个亚区的生态地理单元层级差异不 一致。

\section{4 结论}

该区划在前人动物地理区划的基础上, 将全国 划分为 $5 \mathrm{~km} \times 5 \mathrm{~km}$ 的网格, 对各网格动物分布型 比例及环境因子进行聚类分析, 根据聚类结果进行 了动物地理区划, 并对各区划界线进行了精细化制 图。与张荣祖(2011)区划方案相比, 该区划从生态生 物地理学的角度, 根据各动物地理省内动物生态成 分的差异, 划分了生态地理单元, 并结合野生动物 调查及管理实际, 增加了调查单元, 以满足野生动 物保护管理需要。

致谢: 国家林业局保护司、国家林业局调查规划设 计院、华南师范大学对本次区划工作给予了大力支 持; 张㭉祖先生对区划思路、区划方案和具体方法 均进行了悉心指导, 提供了大量资料, 并对区划方 
案修改提出了建设性意见; 黑龙江省野生动物研究 所、西北濒危动物研究所、中国科学院昆明动物研 究所、四川省林业厅、云南省林业厅等单位专门组 织专家会议, 对区划方案草案进行了研讨; 许多专 家, 如: 胡锦㒹、冯祚建、王应祥、杨大同、杨岗、 韩联宪、江望高、蒋学龙、李保国、李迪强、李林、 刘少英、刘洋、隆廷伦、庆宁、饶定齐、苏化龙、 吴诗宝、吴毅、杨晓君、袁施涁、赵晓东、张明海、 张泽均、赵文阁、钟立成等对区划方案提出了宝贵 意见或提供了大量第一手资料; 各省、自治区、直 辖市野生动物主管部门均结合当地实际, 提出修改 意见或建议; 区划方案形成后，马建章、郑光美、 张荣祖、冯祚建、蒋志刚、张正旺、李保国、杨奇 森、杨晓君、周立志、吴孝兵、钟立成等专家进行 了评审, 并提出了宝贵的意见或建议。在此一并致 以衰心感谢。

\section{参考文献}

Chen L, Song Y, Xu S (2008) The boundary of palaearctic and oriental realms in western China. Progress in Natural Science, 18, 833-841.

De Klerk H, Crowe T, Fjeldså J, Burgess ND (2002) Biogeographical patterns of endemic terrestrial Afrotropical birds. Diversity and Distributions, 8, 147-162.

Fattorini S (2015) On the concept of chorotype. Journal of Biogeography, 42, 2246-2251.

Fattorini S (2016) A history of chorological categories. History and Philosophy of the Life Sciences, 38, 12. https://doi.org/10.1007/s40656-016-0114-1 (accessed on 2017-04-25)

Fei L, Hu SQ, Ye CY, Huang YZ (2006) Fauna Sinica (Amphibia 1): General Accounts of Amphibia, Gymnophiona and Urodela. Science Press, Beijing. (in Chinese) [费梁, 胡 淑琴, 叶昌媛, 黄永昭 (2006) 中国动物志・两栖纲・上卷: 总论, 蚂螈目, 有尾目. 科学出版社, 北京.]

Fei L, Hu SQ, Ye CY, Huang YZ (2009a) Fauna Sinica (Amphibia 2): Anura. Science Press, Beijing. (in Chinese) [费梁, 胡淑琴, 叶昌媛, 黄永昭 (2009a) 中国动物志・两 栖纲・中卷: 无尾目. 科学出版社, 北京.]

Fei L, Hu SQ, Ye CY, Huang YZ (2009b) Fauna Sinica (Amphibia 3): Anura, Ranidae. Science Press, Beijing. (in Chinese) [费梁, 胡淑琴, 叶昌媛, 黄永昭 (2009b) 中国 动物志・两栖纲・下卷: 无尾目, 蛙科. 科学出版社, 北京.]

Feng JM, Zhu YY (2010) On the genera of seed plants endemic to China in Yunnan. Ecology and Environmental Sciences, 19, 621-625. (in Chinese with English abstract) [冯建孟, 朱有勇 (2010) 云南地区中国种子植物特有属的研究.
生态环境学报, 19, 621-625.]

Gao EH, Wang ZC, Wang WS, Chen DF, Ma GQ, Tang XP (2014) Technical plan for the second national survey of terrestrial wildlife in China. Chinese Journal of Wildlife, 35, 238-240. (in Chinese with English abstract) [郜二虎, 王志 臣, 王维胜, 陈涤非, 马国青, 唐小平 (2014) 全国第二 次陆生野生动物资源调查总体思路. 野生动物学报, 35, 238-240.]

Gong ZD, Duan XD, Feng XG, Wu HY, Liu Q (1999) The fauna and ecology of fleas in Cangshan Mountain and Erhai Lake Nature Reserve, Dali. Zoological Research, 20, 451-456. (in Chinese with English abstract) [龚正达, 段兴 德, 冯锡光, 吴厚永, 刘泉 (1999) 大理苍山洱海自然保 护区山地蚤类区系与生态的研究. 动物学研究, 20 , 451-456.]

He J, Kreft H, Gao E, Wang Z, Jiang H (2017) Patterns and drivers of zoogeographical regions of terrestrial vertebrates in China. Journal of Biogeography, 44, 1172-1184.

Hijmans RJ, Cameron SE, Parra JL, Jones PG, Jarvis A (2005) Very high resolution interpolated climate surfaces for global land areas. International Journal of Climatology, 25, 1965-1978.

Hoffmann RS (2001) The southern boundary of the Palaearctic realm in China and adjacent countries. Acta Zoologica Sinica, 47, 121-131.

Holt BG, Lessard JP, Borregaard MK, Fritz SA, Araújo MB, Dimitrov D, Fabre PH, Graham CH, Graves GR, Jønsson KA, Nogués-Bravo D, Wang Z, Whittaker RJ, Fjeldså J, Rahbek C (2013) An update of Wallace's zoogeographic regions of the world. Science, 339, 74-78.

Huang BW (1958) A preliminary draft of comprehensive physical regionalization in China. Acta Geographica Sinica, 24, 348-365. (in Chinese) [黄秉维 (1958) 中国综合自然 区划的初步草案. 地理学报, 24, 348-365.]

Kreft H, Jetz W (2010) A framework for delineating biogeographical regions based on species distributions. Journal of Biogeography, 37, 2029-2053.

Lennon JJ, Koleff P, Greenwood J, Gaston KJ (2001) The geographical structure of British bird distributions: diversity, spatial turnover and scale. Journal of Animal Ecology, 70, 966-979.

Li XC (2000) Multivariate analysis on zoogeographical division. Journal of Northwest University (Natural Science Edition), 30, 265-267. (in Chinese with English abstract) [李晓晨 (2000) 动物地理区划的聚类分析. 西北大学学 报(自然科学版), 30, 265-267.]

Luo KF (1954) Draft of natural geography regionalization of China. Acta Geographica Sinica, 20, 379-394. (in Chinese) [罗开富 (1954) 中国自然地理分区草案. 地理学报, 20, 379-394.]

Passalacqua NG (2015) On the definition of element, chorotype and component in biogeography. Journal of Biogeography, 
42, 611-618.

Ren ME (1999) Outline of China's Physical Geography, 3rd edn. The Commercial Press, Beijing. (in Chinese) [任美锣 (1999) 中国自然地理纲要(第三版). 商务印书馆, 北京.]

Rueda M, Rodríguez MÁ, Hawkins BA (2013) Identifying global zoogeographical regions: lessons from Wallace. Journal of Biogeography, 40, 2215-2225.

Rondinini C, Di Marco M, Chiozza F, Santulli G, Baisero D, Visconti P, Hoffmann M, Schipper J, Stuart SN, Tognelli MF, Amori G, Falcucci A, Maiorano L, Boitani L (2011) Global habitat suitability models of terrestrial mammals. Philosophical Transactions of the Royal Society B: Biological Sciences, 366, 2633-2641.

Shou ZH (1955) The distribution of fur-bearing mammals in China. Acta Geographica Sinica, 21, 405-421. (in Chinese with English abstract) [寿振黄 (1955) 中国毛皮兽的分布. 地理学报, 21, 405-421.]

Sun ZY, Liu SY, Liu Y, Zhao J, Xu MY, Ni G, Yang ZH (2007) Diversity of large and medium sized mammals in the Haizishan Nature Reserve, Sichuan Province. Acta Theriologica Sinica, 27, 274-279. (in Chinese with English abstract) [孙治宇, 刘少英, 刘洋, 赵杰, 徐明友, 倪刚, 杨执衡 (2007) 四川海子山自然保护区大中型兽类多样 性调查. 兽类学报, 27, 274-279.]

Tian HZ, Dong QY, Li HQ (2013) Comparison of Orchidaceae flora in 38 regions of China. Acta Botanica Boreali-Occidentalia Sinica, 33, 808-823. (in Chinese with English abstract) [田怀珍, 董全英, 李宏庆 (2013) 中国38个地区 兰科植物区系成分的比较分析. 西北植物学报, 33, 808-823

Tu FY, Tang MK, Liu Y, Sun ZY, Zhang XY, Yue BS, Liu SY (2012) Fauna and species diversity of small mammals in Jiajin Mountains, Sichuan Province, China. Acta Theriologica Sinica, 32, 287-296. (in Chinese with English abstract) [涂飞云, 唐明坤, 刘洋, 孙治宇, 张修月, 岳碧松, 刘少 英 (2012) 四川夹金山小型兽类区系及多样性. 兽类学 报, 32, 287-296.]

Wallace AR (1876) The Geographical Distribution of Animals. Harper \& Brothers, New York.

Wang YX (2003) A Complete Checklist of Mammal Species and Subspecies in China: A Taxonomic and Geographic Reference. China Forestry Publishing House, Beijing. (in Chinese) [王应祥 (2003) 中国哺乳动物种和亚种分类名 录与分布大全. 中国林业出版社, 北京.]

Xiang Z, Liang X, Huo S, Ma S (2004) Quantitative analysis of land mammal zoogeographical regions in China and adjacent regions. Zoological Studies, 43, 142-160.

Xie Y (2000) Study on Bio-geographical Divisions of China. $\mathrm{PhD}$ dissertation, Institute of Animal, Chinese Academy of Sciences, Beijing. (in Chinese with English abstract) [解炎 (2000) 中国生物地理区划研究. 博士学位论文, 中国科 学院动物研究所, 北京.]
Xie Y, Li DM, MacKinnon J (2002) Preliminary researches on bio-geographical divisions of China. Acta Ecologica Sinica, 22, 1599-1615. (in Chinese with English abstract) [解炎, 李典谟, John MacKinnon (2002) 中国生物地理区划研究. 生态学报, 22, 1599-1615.]

Xie Y, MacKinnon J, Li DM (2004) Study on biogeographical divisions of China. Biodiversity and Conservation, 13, 1391-1417.

Xing Y, Zhou L, Zhang Y, Wang X (2008) Geographical patterns based on faunal types of breeding birds and mammals in China. Integrative Zoology, 3, 280-289.

Zhang MW, Zong Y, Ma JF (1998) Fauna Sinica (Reptilia 1): General Accounts of Reptilia, Testudoformes and Crocodiliformes. Science Press, Beijing. (in Chinese) [张孟闻, 宗愉, 马积藩 (1998) 中国动物志・爬行纲・第一卷: 总论, 龟鳖 目, 鳄形目. 科学出版社, 北京.]

Zhang RZ (2011) China Animal Geography. Science Press, Beijing. (in Chinese) [张荣祖 (2011) 中国动物地理. 科学 出版社, 北京.]

Zhang XS (2007) Vegetation Map of the People's Republic of China $(1: 1,000,000)$. Geological Publishing House, Beijing. (in Chinese) [张新时 (2007) 中华人民共和国植被图 $(1: 1,000,000)$. 地质出版社, 北京.]

Zhang YY, Zhou LZ, Wang QS, Wang XJ, Xing YJ (2008) Distribution pattern and hotspot analysis of breeding birds in Anhui Province. Biodiversity Science, 16, 305-312. (in Chinese with English abstract) [张有瑜, 周立志, 王岐山, 王新建, 邢雅俊 (2008) 安徽省繁殖鸟类分布格局和热 点区分析. 生物多样性, 16, 305-312.]

Zhao EM, Huang MH, Zong Y (1998) Fauna Sinica (Reptilia 3): Squamata (Serpentes). Science Press, Beijing. (in Chinese) [赵尔宓, 黄美华, 宗愉 (1998) 中国动物志・爬 行纲・第三卷: 有鳞目, 蛇亚目. 科学出版社, 北京.]

Zhao EM, Zhao KT, Zhou KY (1999) Fauna Sinica (Reptilia 2): Squamata (Lacertilia). Science Press, Beijing. (in Chinese) [赵尔宓, 赵肯堂, 周开亚 (1999) 中国动物志・ 爬行纲・第二卷: 有鳞目, 蚚蜴亚目. 科学出版社, 北京.]

Zhao SQ (1983) A new scheme for comprehensive physicalgeopraphical regionalization in China. Acta Geopraphica Sinica, 38, 1-10. (in Chinese with English abstract) [赵松乔 (1983) 中国综合自然地理区划的一个新方案. 地理学报, $38,1-10$.]

Zheng D (2008) China's Ecogeographical Regionalization Research. The Commercial Press, Beijing. (in Chinese) [郑度 (2008) 中国生态地理区域系统研究. 商务印书馆, 北京.]

Zheng GM (2011) A Checklist on the Classification and Distribution of the Birds of China, 2nd edn. Science Press, Beijing. [郑光美 (2011) 中国鸟类分类与分布名录(第二 版). 科学出版社, 北京.]

Zheng ZX, Zhang RZ (1959) China animal geographical regionalization. In: China Animal Geographical Regionalization and China Insects Geographical Regionalization (draft) (ed. 
Committee of Natural Regionalization of Chinese Academy of Sciences), pp. 1-66. Science Press, Beijing. (in Chinese) [郑作新, 张荣祖 (1959) 中国动物地理区划. 见: 中国动 物地理区划与中国昆虫地理区划(初稿) (中国科学院自然
区划工作委员会编), 1-66页. 科学出版社, 北京.]

(责任编委：江建平 责任编辑：问文杰)

\section{附录 Supplementary Material}

附录1 用于区划的物种分布参考文献

Appendix 1 References about species distribution for regionalization

http://www.biodiversity-science.net/fileup/PDF/2017135-1.pdf

附录 2 未用于区划的物种数量

Appendix 2 Number of species not involved in the regionalization

http://www.biodiversity-science.net/fileup/PDF/2017135-2.pdf

附录 3 用于区划的物种数量

Appendix 3 Number of species involved in the regionalization http://www.biodiversity-science.net/fileup/PDF/2017135-3.pdf

附录 4 物种适生栖息地模型示意图

Appendix 4 Framework of the habitat suitability models http://www.biodiversity-science.net/fileup/PDF/2017135-4.pdf

附录 5 用于区划的物种名录及分布型

Appendix 5 Checklist of species involved in the regionalization and their chorotypes http://www.biodiversity-science.net/fileup/PDF/2017135-5.pdf

附录 6 分布型代码

Appendix 6 Code of chorotype

http://www.biodiversity-science.net/fileup/PDF/2017135-6.pdf

附录 7 环境指标及数据来源

Appendix 7 Environmental index and data resources

http://www.biodiversity-science.net/fileup/PDF/2017135-7.pdf

附录 8 全国陆生野生动物调查单元区划表

Appendix 8 Table of zoogeographical regions of China for terrestrial wildlife survey http://www.biodiversity-science.net/fileup/PDF/2017135-8.pdf 
郜二虎, 何杰坤, 王志臣, 徐扬, 唐小平, 江海声. 全国陆生野生动物调查单元区划方案. 生物多样性, 2017, 25 (12): 1321-1330.

http://www.biodiversity-science.net/CN/10.17520/biods.2017135

附录 1 用于区划的物种分布参考文献

Appendix 1 References about species distribution for regionalization

Dang KL, Li DW, Wang KF (2006) Comprehensive Scientific Survey and Biodiversity Research on Guanyinshan Nature Reserve in Shaanxi. China Forestry Publishing House, Beijing. (in Chinese) [党坤良, 李登武, 王开锋 (2006) 陕西观音山自然保护区综合科学考 察与生物多样性研究. 中国林业出版社, 北京.]

Fei L, Hu SQ, Ye CY, Huang YZ (2006) Fauna Sinica (Amphibia 1): General Accounts of Amphibia, Gymnophiona and Urodela. Science Press, Beijing. (in Chinese) [费梁, 胡 淑琴，叶昌媛，黄永昭 (2006) 中国动物志·两栖纲. 上卷: 总论, 蚓螈目, 有尾目. 科学出版社, 北京.]

Fei L, Hu SQ, Ye CY, Huang YZ (2009a) Fauna Sinica (Amphibia 2): Anura. Science Press, Beijing. (in Chinese) [费梁, 胡淑琴, 叶昌媛, 黄永昭 (2009a) 中国动物志・两栖纲. 中卷: 无尾目. 科学出版社, 北京.]

Fei L, Hu SQ, Ye CY, Huang YZ (2009b) Fauna Sinica (Amphibia 3): Anura, Ranidae. Science Press, Beijing. (in Chinese) [费梁，胡淑琴，叶昌媛，黄永昭 (2009b) 中国动物志·两 栖纲. 下卷: 无尾目, 蛙科. 科学出版社, 北京.]

Fei L, Ye CY (2001) The Colour Handbook of the Amphibians of Sichuan. China Forestry Publishing House, Beijing. (in Chinese) [费梁，叶昌媛 (2001) 四川两栖类原色图鉴. 中国林业出版社，北京.]

Fei L, Ye CY, Jiang JP (2010) Colored Atlas of Chinese Amphibians. Sichuan Science and Technology Press, Chengdu. (in Chinese) [费梁, 叶昌媛, 江建平 (2010) 中国两栖动 物彩色图鉴. 四川科学技术出版社, 成都.]

Feng ZJ, Cai GQ, Zheng CL (1986) Mammals of Tibet. Science Press, Beijing. (in Chinese) [冯祚 建, 蔡桂全, 郑昌琳 (1986) 西藏哺乳类. 科学出版社, 北京.]

Gong MH, Lin C, Ge JW, Zhang HM, Liu XS, Guan LF, Lou D (2011) Scientific Survey and Research on the Shibalichangxia Nature Reserve in Hubei, China. Beijing Publishing House, Beijing. (in Chinese) [龚明吴, 萄琛, 葛继稳, 张洪茂, 刘绪生, 关良福, 楼丹 (2011) 湖北十八里长峡自然保护区科学考察与研究. 北京出版社, 北京.]

Gong MH, Liu ZL, Zhang JJ, Guan TP, Yan WB, Liu Y, Xu ZC, Ju XZ (2011) Scientific Survey 
郜二虎, 何杰坤, 王志臣, 徐扬, 唐小平, 江海声. 全国陆生野生动物调查单元区划方案. 生物多样性, 2017, 25 (12): 1321-1330.

http://www.biodiversity-science.net/CN/10.17520/biods.2017135

and Research on Daheishan Nature Reserve in Liaoning, China. Beijing Publishing House, Beijing. (in Chinese) [龚明吴，刘增力，张建军，官天培，颜文博，刘洋，徐振 财，鞠秀芝 (2011) 辽宁大黑山自然保护区科学考察与研究. 北京出版社，北京.]

Huang GL (2009) Qinghai Kelukehu-Tuosuhu Wetland Nature Reserve Biodiversity. Beijing Publishing House, Beijing. (in Chinese) [黄桂林 (2009) 青海可鲁克湖-托素湖湿地 自然保护区生物多样性. 北京出版社，北京.]

Jiang ZG (2006) Biodiversity in the Laoxiancheng Nature Reserve, Shaanxi, China. Tsinghua University Press, Beijing. (in Chinese) [蒋志刚 (2006) 陕西老县城自然保护区的生 物多样性. 清华大学出版社, 北京.]

Lei FM, Lu TC (2006) China’s Endemic Birds. Science Press, Beijing. (in Chinese) [雷富民, 卢 汰春 (2006) 中国鸟类特有种. 科学出版社, 北京.]

Liu GQ, Wang ZC, Ma GQ (2000) Beijing Yunmengshan Nature Reserve Biodiversity. Beijing Publishing House, Beijing. (in Chinese) [刘国强, 王志臣, 马国青 (2000) 北京云蒙 山自然保护区生物多样性. 北京出版社, 北京.]

Liu ZL, Yue JB, Song TF (2009) Liaoning Zhanggutai Nature Reserve Biodiversity. Beijing Publishing House, Beijing. (in Chinese) [刘增力, 岳建兵, 宋铁芳 (2009) 辽宁章古 台自然保护区生物多样性. 北京出版社，北京.]

Peng YZ, Yang DH, Kuang BY (1987) Bird List of Yunnan. Yunnan Science and Technology Press, Kunming. (in Chinese) [彭燕章, 杨德华, 匡邦郁 (1987) 云南鸟类名录. 云南科技 出版社, 昆明.]

Shi HT, Zhao EM, Wang LJ (2011) Amphibia and Reptile Fauna of Hainan. Science Press, Beijing. (in Chinese) [史海涛, 赵尔宓, 王力军 (2011) 海南两栖爬行动物志. 科学出版社, 北京.]

State Forestry Administration of the People's Republic of China (2009) Report of Important Terrestrial Wildlife Resource Survey in China. China Forestry Publishing House, Beijing. [国家林业局 (2009) 中国重点陆生野生动物资源调查. 中国林业出版社, 北京.]

Tang XP, Wang ZC, Zhang MX (2006) Henan Danjiang Wetland Nature Reserve Biodiversity. Beijing Publishing House, Beijing. (in Chinese) [唐小平, 王志臣, 张明祥 (2006) 河 
郜二虎, 何杰坤, 王志臣, 徐扬, 唐小平, 江海声. 全国陆生野生动物调查单元区划方案. 生物多样性, 2017, 25 (12): 1321-1330.

http://www.biodiversity-science.net/CN/10.17520/biods.2017135

南丹江湿地自然保护区生物多样性. 北京出版社, 北京.]

Wang XT (1991) Vertebrate Fauna of Gansu. Gansu Science and Technology Press, Lanzhou. (in Chinese) [王香亭 (1991) 甘肃省脊椎动物志. 甘肃科学技术出版社, 兰州.]

Wang YX (2003) A Complete Checklist of Mammal Species and Subspecies in China: A Taxonomic and Geographic Reference. China Forestry Publishing House, Beijing. (in Chinese) [王应祥 (2003) 中国哺乳动物种和亚种分类名录与分布大全. 中国林业 出版社，北京.]

Wang ZC, Ma GQ, Wang JZ (2009) Beijing Baihuashan National Nature Reserve Biodiversity. Beijing Publishing House, Beijing. (in Chinese) [王志臣, 马国青, 王九中 (2009) 北 京百花山国家级自然保护区生物多样性研究. 北京出版社, 北京.]

Weng GQ, Li Y (2006) Neimenggu Hatengtaohai National Nature Reserve Biodiversity. Beijing Publishing House, Beijing. (in Chinese) [翁国庆, 李燕 (2006) 内蒙古哈腾套海国家 级自然保护区生物多样性. 北京出版社, 北京.]

Yang DT (1991) The Amphibia Fauna of Yunnan. China Forestry Publishing House, Beijing. (in Chinese) [杨大同 (1991) 云南两栖类志. 中国林业出版社，北京.]

Yu BC, Liang BK, Li YB, Xue YG, Yang CS, Zhang JJ, Liu Y (2012) Scientific Survey and Research on Daguishan Chinese Xenosaurs Nature Reserve in Guangxi, China. Beijing Publishing House, Beijing. (in Chinese) [遇宝成, 梁兵宽, 李友邦, 薛跃规, 阳春生, 张建军, 刘洋 (2012) 广西大桂山鳄蚚自然保护区科学考察与研究. 北京出版社, 北京.]

Zhang JJ (2009) Gansu Xiaolongshan National Nature Reserve Biodiversity. Beijing Publishing House, Beijing. (in Chinese) [张建军 (2009) 甘肃小陇山国家级自然保护区生物多 样性. 北京出版社, 北京.]

Zhang MW, Zong Y, Ma JF (1998) Fauna Sinica (Reptilia 1): General Accounts of Reptilia, Testudoformes and Crocodiliformes. Science Press, Beijing. (in Chinese) [张孟闻, 宗 愉, 马积藩 (1998) 中国动物志・爬行纲・第一卷: 总论, 龟鳖目, 鳄形目. 科学出版 社, 北京.]

Zhang RZ (1997) Distribution of Mammalian Species in China. China Forestry Publishing House, Beijing. (in Chinese) [张荣祖 (1997) 中国哺乳动物分布. 中国林业出版社，北京.] 
郜二虎, 何杰坤, 王志臣, 徐扬, 唐小平, 江海声. 全国陆生野生动物调查单元区划方案. 生物多样性, 2017, 25 (12): 1321-1330.

http://www.biodiversity-science.net/CN/10.17520/biods.2017135

Zhao EM, Huang MH, Zong Y (1999) Fauna Sinica (Reptilia 3): Squamata (Serpentes). Science Press, Beijing. (in Chinese) [赵尔宓, 黄美华, 宗愉 (1999) 中国动物志・爬行纲・第三 卷: 有鳞目, 蛇亚目. 科学出版社, 北京.]

Zhao EM, Jiang YM, Huang QY, Zhao H, Zhao KT, Zhou KY, Liu YZ, Liu MY, Li DJ, Zhang YX (1998) Fauna Sinica (Reptilia 2): Squamata (Lacertilia). Science Press, Beijing. (in Chinese) [赵尔宓，江耀明，黄庆云，赵惠，赵肯堂，周开亚，刘月珍，刘明玉，李德 俊，张玉霞 (1998) 中国动物志・爬行纲・第二卷: 有鳞目，蚚蜴亚目. 科学出版社, 北京.]

Zhao ZJ (2001a) A Handbook of the Birds of China (Volume I): Non-passerines. Jilin Science and Technology Press, Changchun. (in Chinese) [赵正阶 (2001a) 中国鸟类志 (上卷): 非 雀形目. 吉林科学技术出版社, 长春.]

Zhao ZJ (2001b) A Handbook of the Birds of China (Volume II): Passerines. Jilin Science and Technology Press, Changchun. (in Chinese) [赵正阶 (2001b) 中国鸟类志 (下卷): 雀 形目. 吉林科学技术出版社, 长春.]

Zheng GM (2011) A Checklist on the Classification and Distribution of the Birds of China, 2nd edn. Science Press, Beijing. [郑光美 (2011) 中国鸟类分类与分布名录(第二版). 科 学出版社, 北京.] 
郜二虎, 何杰坤, 王志臣, 徐扬, 唐小平, 江海声. 全国陆生野生动物调查单元区划方案. 生物多样性, 2017, 25 (12): 1321-1330.

http://www.biodiversity-science.net/CN/10.17520/biods.2017135

附录 2 末用于区划的物种数量

Appendix 2 Number of species not involved in the regionalization

\begin{tabular}{llll}
\hline 纲 & 主动剔除的物种数量 & 资料不详的物种数量 & 总计 \\
Class & Number of species removed & $\begin{array}{l}\text { Number of species whose } \\
\text { distributions are not clear }\end{array}$ & Total \\
\hline 两栖纲 Amphibia & 80 & 11 & 91 \\
爬行纲 Reptilia & 9 & 11 & 20 \\
鸟纲 Aves & 229 & 329 & 558 \\
哺乳纲 Mammalia & 198 & 17 & 215 \\
总计 Total & 516 & 368 & 884 \\
\hline
\end{tabular}


郜二虎, 何杰坤, 王志臣, 徐扬, 唐小平, 江海声. 全国陆生野生动物调查单元区划方案. 生物多样性, 2017, 25 (12): 1321-1330.

http://www.biodiversity-science.net/CN/10.17520/biods.2017135

附录 3 用于区划的物种数量

Appendix 3 Number of species involved in the regionalization

\begin{tabular}{lllll}
\hline 纲 & 目 & 科 & 属 & 种 \\
Class & Oder & Family & Genus & Species \\
\hline 两栖纲 Amphibia & 3 & 11 & 59 & 262 \\
爬行纲 Reptilia & 3 & 21 & 109 & 358 \\
鸟纲 Aves & 21 & 87 & 335 & 814 \\
哺乳纲 Mammalia & 10 & 35 & 164 & 350 \\
总计 Total & 37 & 154 & 332 & 1,784 \\
\hline
\end{tabular}


郜二虎, 何杰坤, 王志臣, 徐扬, 唐小平, 江海声. 全国陆生野生动物调查单元区划方案. 生物多样性, 2017, 25 (12): 1321-1330.

http://www.biodiversity-science.net/CN/10.17520/biods.2017135

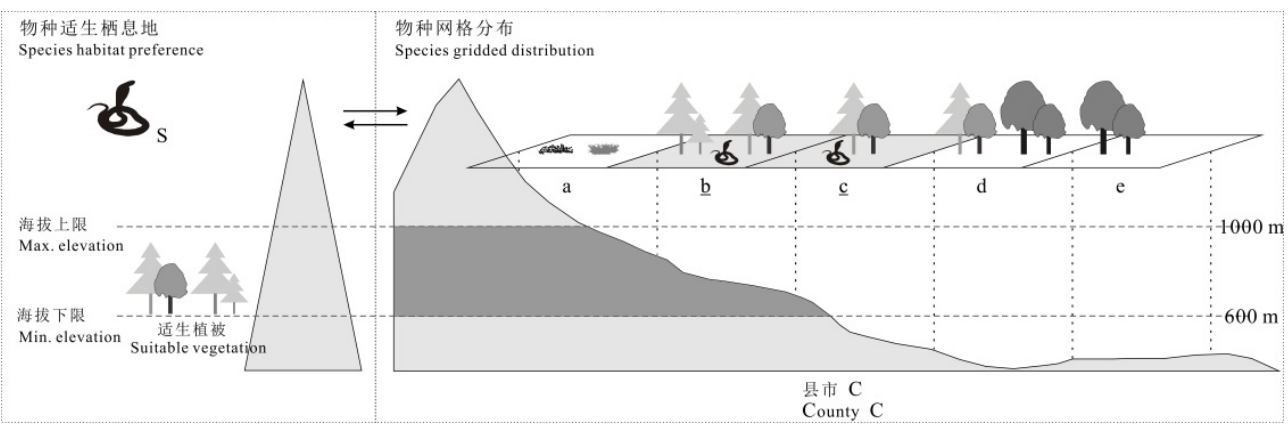

附录 4 物种适生栖息地模型示意图。*假设物种 $\mathrm{S}$ 的适生栖息地为针叶林和针阔混交林, 分布海拔为 600-1,000 m, 并在C县市(网格 $\mathrm{a}, \mathrm{b}, \mathrm{c}, \mathrm{d}$ 和e)有分布记录。网格a, b 和c介于海拔 600-1,000 m之间, 网格 b, c和 $d$ 分布有针叶林或者针阔混交林。因此, 只有网格 $\mathrm{b}$ 和网格 $\mathrm{c}$ 同时符合物种 $\mathrm{S}$ 的栖息地环境及海拔范围, 因而认 为物种 $S$ 在网格 $b$ 和c有分布, 在网格 $a, d, e$ 没有分布。

Appendix 4 Framework of the habitat suitability models. *For example, the suitable vegetation for species $\mathrm{S}$ is coniferous forests and coniferous broad-leaved forests, with an altitude of $600-1,000 \mathrm{~m}$. Species $\mathrm{S}$ is recorded in county $\mathrm{C}$, which is divided into grid $\mathrm{a}, \mathrm{b}, \mathrm{c}, \mathrm{d}$ and e. In these grids, grid $\mathrm{a}, \mathrm{b}$ and c have some areas of altitude of 600-1,000 m, while grid b, c and d have vegetation of coniferous forests and/or coniferous broad-leaved forests. So, grid $\mathrm{b}$ and $\mathrm{c}$ are considered suitable for species $\mathrm{S}$, because only grid $\mathrm{b}$ and $\mathrm{c}$ have suitable habitat and elevation for species $\mathrm{S}$, while grid $\mathrm{a}, \mathrm{d}$, and e are not. 
郜二虎, 何杰坤, 王志臣, 徐扬, 唐小平, 江海声. 全国陆生野生动物调查单元区划方案. 生物多样性, 2017, 25 (12): 1321-1330.

http://www.biodiversity-science.net/CN/10.17520/biods.2017135

附录 5 用于区划的物种名录及分布型

Appendix 5 Checklist of species involved in the regionalization and their chorotypes

\begin{tabular}{|c|c|c|c|c|c|c|c|c|c|c|}
\hline $\begin{array}{r}\text { 序号 } \\
\text { No. }\end{array}$ & \multicolumn{2}{|c|}{$\begin{array}{c}\text { 纲 } \\
\text { Class }\end{array}$} & \multicolumn{2}{|c|}{$\begin{array}{c}\text { 目 } \\
\text { Order }\end{array}$} & \multicolumn{2}{|r|}{$\begin{array}{c}\text { 科 } \\
\text { Family }\end{array}$} & \multicolumn{2}{|r|}{$\begin{array}{l}\text { 物种名 } \\
\text { Species }\end{array}$} & \multirow{2}{*}{$\begin{array}{l}\text { 分布型 } \\
\text { Chorotype }\end{array}$} & \multirow[t]{2}{*}{$\begin{array}{c}\text { 备注 } \\
\text { Remarks }\end{array}$} \\
\hline 1 & 两栖纲 & Amphibia & 蚓螈目 & Gymnophiona & 鱼螈科 & Ichthyophiidae & 版纳鱼螈 & Ichthyophis bannanicus & & \\
\hline 2 & 两栖纲 & Amphibia & 有尾目 & Urodela & 小鲵科 & Hynobiidae & 阿里山小鲵 & Hynobius arisanensis & $\mathrm{j}$ & \\
\hline 3 & 两栖纲 & Amphibia & 有尾目 & Urodela & 小鲵科 & Hynobiidae & 台湾小鲵 & Hynobius formosanus & $\mathrm{j}$ & \\
\hline 4 & 两栖纲 & Amphibia & 有尾目 & Urodela & 小鲵科 & Hynobiidae & 楚南小鲵 & Hynobius soanni & $\mathrm{j}$ & \\
\hline 5 & 两栖纲 & Amphibia & 有尾目 & Urodela & 小鲵科 & Hynobiidae & 安吉小鲵 & Hynobius amjiensis & si & \\
\hline 6 & 两栖纲 & Amphibia & 有尾目 & Urodela & 小鲵科 & Hynobiidae & 中国小鲵 & Hynobius chinensis & si & \\
\hline 7 & 两栖纲 & Amphibia & 有尾目 & Urodela & 小鲵科 & Hynobiidae & 东北小鲵 & Hynobius leechii & $\mathrm{m}$ & \\
\hline 8 & 两栖纲 & Amphibia & 有尾目 & Urodela & 小鲵科 & Hynobiidae & 义乌小鲵 & Hynobius yiwuensis & si & \\
\hline 9 & 两栖纲 & Amphibia & 有尾目 & Urodela & 小鲵科 & Hynobiidae & 商城肥鲵 & Pachyhynobius shangchengensis & sh & \\
\hline 10 & 两栖纲 & Amphibia & 有尾目 & Urodela & 小鲵科 & Hynobiidae & 极北鲵 & Salamandrella keyserlingii & u & \\
\hline 11 & 两栖纲 & Amphibia & 有尾目 & Urodela & 小鲵科 & Hynobiidae & 秦巴拟小鲵 & Pseudohynobius tsinpaensis & sh & \\
\hline 12 & 两栖纲 & Amphibia & 有尾目 & Urodela & 小鲵科 & Hynobiidae & 爪鲵 & Onychodactylus fischeri & $\mathrm{mf}$ & 现分为 2 种 \\
\hline 13 & 两栖纲 & Amphibia & 有尾目 & Urodela & 小鲵科 & Hynobiidae & 巫山北鲵 & Ranodon shihi & sn & \\
\hline 14 & 两栖纲 & Amphibia & 有尾目 & Urodela & 小鲵科 & Hynobiidae & 新疆北鲵 & Ranodon sibiricus & df & \\
\hline 15 & 两栖纲 & Amphibia & 有尾目 & Urodela & 小鲵科 & Hynobiidae & 龙洞山溪鲵 & Batrachuperus londongensis & hc & \\
\hline 16 & 两栖纲 & Amphibia & 有尾目 & Urodela & 小鲵科 & Hynobiidae & 山溪鲵 & Batrachuperus pinchonii & hc & \\
\hline 17 & 两栖纲 & Amphibia & 有尾目 & Urodela & 小鲵科 & Hynobiidae & 西藏山溪鯢 & Batrachuperus tibetanus & hc & \\
\hline 18 & 两栖纲 & Amphibia & 有尾目 & Urodela & 小鲵科 & Hynobiidae & 盐源山溪鲵 & Batrachuperus yenyuanensis & hc & \\
\hline 19 & 两栖纲 & Amphibia & 有尾目 & Urodela & 隐鳃鲵科 & Cryptobranchidae & 大鲵 & Andrias davidianus & $\mathrm{e}$ & \\
\hline 20 & 两栖纲 & Amphibia & 有尾目 & Urodela & 蝶螈科 & Salamandroidae & 细痣疮螈 & Tylototriton asperrimus & sc & \\
\hline
\end{tabular}


郜二虎, 何杰坤, 王志臣, 徐扬, 唐小平, 江海声. 全国陆生野生动物调查单元区划方案. 生物多样性, 2017, 25 (12): 1321-1330.

http://www.biodiversity-science.net/CN/10.17520/biods.2017135

\begin{tabular}{|c|c|c|c|c|c|c|c|c|c|c|}
\hline \multirow{2}{*}{$\begin{array}{l}\text { 序号 } \\
\text { No. } \\
21\end{array}$} & \multicolumn{2}{|c|}{$\begin{array}{c}\text { 纲 } \\
\text { Class }\end{array}$} & \multicolumn{2}{|c|}{$\begin{array}{c}\text { 目 } \\
\text { Order }\end{array}$} & \multicolumn{2}{|r|}{$\begin{array}{c}\text { 科 } \\
\text { Family }\end{array}$} & \multicolumn{2}{|r|}{$\begin{array}{l}\text { 物种名 } \\
\text { Species }\end{array}$} & \multirow{2}{*}{$\begin{array}{l}\begin{array}{c}\text { 分布型 } \\
\text { Chorotype }\end{array} \\
\mathrm{j}\end{array}$} & \multirow[t]{2}{*}{$\begin{array}{c}\text { 备注 } \\
\text { Remarks }\end{array}$} \\
\hline & 两栖纲 & Amphibia & 有尾目 & Urodela & 蝾螈科 & Salamandroidae & 海南疮缐 & Tylototriton hainanensis & & \\
\hline 22 & 两栖纲 & Amphibia & 有尾目 & Urodela & 蝾螈科 & Salamandroidae & 贵州疮螈 & Tylototriton kweichowensis & he & \\
\hline 23 & 两栖纲 & Amphibia & 有尾目 & Urodela & 蝾螈科 & Salamandroidae & 大凉疮螈 & Tylototriton taliangensis & hc & \\
\hline 24 & 两栖纲 & Amphibia & 有尾目 & Urodela & 蝾螈科 & Salamandroidae & 棕黑疮螈 & Tylototriton verrucosus & hc & \\
\hline 25 & 两栖纲 & Amphibia & 有尾目 & Urodela & 蝾螈科 & Salamandroidae & 琉球棘螈 & Echinotriton andersoni & $\mathrm{j}$ & \\
\hline 26 & 两栖纲 & Amphibia & 有尾目 & Urodela & 蝶螈科 & Salamandroidae & 镇海欶螈 & Echinotriton chinhaiensis & si & \\
\hline 27 & 两栖纲 & Amphibia & 有尾目 & Urodela & 蝾螈科 & Salamandroidae & 尾斑㾮虾 & Paramesotriton caudopunctatus & y & \\
\hline 28 & 两栖纲 & Amphibia & 有尾目 & Urodela & 蝾螈科 & Salamandroidae & 中国瘰虾 & Paramesotriton chinensis & sc & \\
\hline 29 & 两栖纲 & Amphibia & 有尾目 & Urodela & 蝾螈科 & Salamandroidae & 广西癝虾 & Paramesotriton guanxiensis & sa & \\
\hline 30 & 两栖纲 & Amphibia & 有尾目 & Urodela & 蝾螈科 & Salamandroidae & 黑斑肥螈 & Pachytriton brevipes & si & \\
\hline 31 & 两栖纲 & Amphibia & 有尾目 & Urodela & 蝾螈科 & Salamandroidae & 无斑肥螈 & Pachytriton labiatus & sc & \\
\hline 32 & 两栖纲 & Amphibia & 有尾目 & Urodela & 蝶螈科 & Salamandroidae & 呈贡蝶螈 & Cynops chenggongensis & y & \\
\hline 33 & 两栖纲 & Amphibia & 有尾目 & Urodela & 蝾螈科 & Salamandroidae & 蓝尾蝾螈 & Cynops cyanurus & $\mathrm{y}$ & \\
\hline 34 & 两栖纲 & Amphibia & 有尾目 & Urodela & 蝾螈科 & Salamandroidae & 东方蝾螈 & Cynops orientalis & $\mathrm{se}$ & \\
\hline 35 & 两栖纲 & Amphibia & 有尾目 & Urodela & 蝾螈科 & Salamandroidae & 滇螈 & Hypselotriton wolterstorffi & $\mathrm{y}$ & \\
\hline 36 & 两栖纲 & Amphibia & 无尾目 & Anura & 铃蟾科 & Bombinatoridae & 东方铃蟾 & Bombina orientalis & $\mathrm{mf}$ & \\
\hline 37 & 两栖纲 & Amphibia & 无尾目 & Anura & 铃蟾科 & Bombinatoridae & 强婚刺铃蟾 & Bombina fortinuptialis & sg & \\
\hline 38 & 两栖纲 & Amphibia & 无尾目 & Anura & 铃蟾科 & Bombinatoridae & 利川铃蟾 & Bombina lichuanensis & si & \\
\hline 39 & 两栖纲 & Amphibia & 无尾目 & Anura & 铃蟾科 & Bombinatoridae & 大蹼铃蟾 & Bombina maxima & hc & \\
\hline 40 & 两栖纲 & Amphibia & 无尾目 & Anura & 铃蟾科 & Bombinatoridae & 微蹼铃蟾 & Bombina microdeladigitora & hc & \\
\hline 41 & 两栖纲 & Amphibia & 无尾目 & Anura & 角蟾科 & Megophryidae & 棘疮齿蟾 & Oreolalax granulosus & hc & \\
\hline 42 & 两栖纲 & Amphibia & 无尾目 & Anura & 角蛻科 & Megophryidae & 景东齿蟾 & Oreolalax jingdongensis & he & \\
\hline
\end{tabular}


郜二虎, 何杰坤, 王志臣, 徐扬, 唐小平, 江海声. 全国陆生野生动物调查单元区划方案. 生物多样性, 2017, 25 (12): 1321-1330.

http://www.biodiversity-science.net/CN/10.17520/biods.2017135

\begin{tabular}{|c|c|c|c|c|c|c|c|c|c|c|}
\hline \multirow{2}{*}{$\begin{array}{l}\text { 序号 } \\
\text { No. } \\
43\end{array}$} & \multicolumn{2}{|c|}{$\begin{array}{c}\text { 纲 } \\
\text { Class }\end{array}$} & \multicolumn{2}{|c|}{$\begin{array}{c}\text { 目 } \\
\text { Order }\end{array}$} & \multicolumn{2}{|r|}{$\begin{array}{c}\text { 科 } \\
\text { Family }\end{array}$} & \multicolumn{2}{|r|}{$\begin{array}{l}\text { 物种名 } \\
\text { Species }\end{array}$} & \multirow{2}{*}{$\begin{array}{l}\text { 分布型 } \\
\text { Chorotype } \\
\text { hc }\end{array}$} & \multirow[t]{2}{*}{$\begin{array}{c}\text { 备注 } \\
\text { Remarks }\end{array}$} \\
\hline & 两栖纲 & Amphibia & 无尾目 & Anura & 角蟾科 & Megophryidae & 疮刺齿蟾 & Oreolalax rugosus & & \\
\hline 44 & 两栖纲 & Amphibia & 无尾目 & Anura & 角蟾科 & Megophryidae & 乡城齿蟾 & Oreolalax xiangchengensis & he & \\
\hline 45 & 两栖纲 & Amphibia & 无尾目 & Anura & 角蛤科 & Megophryidae & 川北齿蟾 & Oreolalax chuanbeiensis & hc & \\
\hline 46 & 两栖纲 & Amphibia & 无尾目 & Anura & 角蛿科 & Megophryidae & 大齿蟾 & Oreolalax major & hc & \\
\hline 47 & 两栖纲 & Amphibia & 无尾目 & Anura & 角蟾科 & Megophryidae & 魏氏齿蟾 & Oreolalax weigoldi & hc & \\
\hline 48 & 两栖纲 & Amphibia & 无尾目 & Anura & 角蛤科 & Megophryidae & 秉志齿蟾 & Oreolalax pingii & hc & \\
\hline 49 & 两栖纲 & Amphibia & 无尾目 & Anura & 角蟾科 & Megophryidae & 普雄齿蟾 & Oreolalax puxiongensis & hc & \\
\hline 50 & 两栖纲 & Amphibia & 无尾目 & Anura & 角蟾科 & Megophryidae & 无蹼齿蟾 & Oreolalax schmidti & hc & \\
\hline 51 & 两栖纲 & Amphibia & 无尾目 & Anura & 角蛿科 & Megophryidae & 利川齿蟾 & Oreolalax lichuanensis & $\mathrm{y}$ & \\
\hline 52 & 两栖纲 & Amphibia & 无尾目 & Anura & 角蟾科 & Megophryidae & 峨眉齿蟾 & Oreolalax omeimontis & hc & \\
\hline 53 & 两栖纲 & Amphibia & 无尾目 & Anura & 角蛿科 & Megophryidae & 宝兴齿蟾 & Oreolalax popei & hc & \\
\hline 54 & 两栖纲 & Amphibia & 无尾目 & Anura & 角蛿科 & Megophryidae & 红点齿蟾 & Oreolalax rhodostigmatus & y & \\
\hline 55 & 两栖纲 & Amphibia & 无尾目 & Anura & 角蟾科 & Megophryidae & 金顶齿突蟾 & Scutiger chintingensis & hc & \\
\hline 56 & 两栖纲 & Amphibia & 无尾目 & Anura & 角蛿科 & Megophryidae & 平武齿突蟾 & Scutiger pingwuensis & hc & \\
\hline 57 & 两栖纲 & Amphibia & 无尾目 & Anura & 角蛿科 & Megophryidae & 西藏齿突蟾 & Scutiger boulengeri & $\mathrm{hm}$ & \\
\hline 58 & 两栖纲 & Amphibia & 无尾目 & Anura & 角蟾科 & Megophryidae & 六盘齿突蟾 & Scutiger liupanensis & $\mathrm{b}$ & \\
\hline 59 & 两栖纲 & Amphibia & 无尾目 & Anura & 角蟾科 & Megophryidae & 宁陕齿突蟾 & Scutiger ningshanensis & sn & \\
\hline 60 & 两栖纲 & Amphibia & 无尾目 & Anura & 角蟢科 & Megophryidae & 花齿突蟾 & Scutiger maculatus & hc & \\
\hline 61 & 两栖纲 & Amphibia & 无尾目 & Anura & 角蟾科 & Megophryidae & 林芝齿突蟾 & Scutiger nyingchiensis & ha & \\
\hline 62 & 两栖纲 & Amphibia & 无尾目 & Anura & 角蟾科 & Megophryidae & 锡金齿突蟾 & Scutiger sikimmensis & ha & \\
\hline 63 & 两栖纲 & Amphibia & 无尾目 & Anura & 角蟾科 & Megophryidae & 贡山猫眼蟾 & Scutiger gongshanensis & hc & \\
\hline 64 & 两栖纲 & Amphibia & 无尾目 & Anura & 角蛻科 & Megophryidae & 刺胸猫眼蟾 & Scutiger mammatus & hc & \\
\hline
\end{tabular}


郜二虎, 何杰坤, 王志臣, 徐扬, 唐小平, 江海声. 全国陆生野生动物调查单元区划方案. 生物多样性, 2017, 25 (12): 1321-1330.

http://www.biodiversity-science.net/CN/10.17520/biods.2017135

\begin{tabular}{|c|c|c|c|c|c|c|c|c|c|c|}
\hline \multirow{2}{*}{$\begin{array}{l}\text { 序号 } \\
\text { No. } \\
65\end{array}$} & \multicolumn{2}{|c|}{$\begin{array}{c}\text { 纲 } \\
\text { Class }\end{array}$} & \multicolumn{2}{|c|}{$\begin{array}{c}\text { 目 } \\
\text { Order }\end{array}$} & \multicolumn{2}{|r|}{$\begin{array}{c}\text { 科 } \\
\text { Family }\end{array}$} & \multicolumn{2}{|r|}{$\begin{array}{l}\text { 物种名 } \\
\text { Species }\end{array}$} & \multirow{2}{*}{$\begin{array}{l}\text { 分布型 } \\
\text { Chorotype } \\
\text { hc }\end{array}$} & \multirow[t]{2}{*}{$\begin{array}{c}\text { 备注 } \\
\text { Remarks }\end{array}$} \\
\hline & 两栖纲 & Amphibia & 无尾目 & Anura & 角蟾科 & Megophryidae & 木里猫眼蟾 & Scutiger muliensis & & \\
\hline 66 & 两栖纲 & Amphibia & 无尾目 & Anura & 角蟾科 & Megophryidae & 胸腺猫眼蟾 & Scutiger glandulatus & he & \\
\hline 67 & 两栖纲 & Amphibia & 无尾目 & Anura & 角蟾科 & Megophryidae & 圆病猫眼蟾 & Scutiger tuberculatus & hc & \\
\hline 68 & 两栖纲 & Amphibia & 无尾目 & Anura & 角蟾科 & Megophryidae & 沙巴拟髭蟾 & Leptobrachium chapaense & hc & \\
\hline 69 & 两栖纲 & Amphibia & 无尾目 & Anura & 角蟾科 & Megophryidae & 海南拟髭蟾 & Leptobrachium hainanense & wa & \\
\hline 70 & 两栖纲 & Amphibia & 无尾目 & Anura & 角蟾科 & Megophryidae & 哀牢髭蟾 & Vibrissaphora ailaonica & hc & \\
\hline 71 & 两栖纲 & Amphibia & 无尾目 & Anura & 角蟾科 & Megophryidae & 峨眉髭蟾 & Vibrissaphora boringii & si & \\
\hline 72 & 两栖纲 & Amphibia & 无尾目 & Anura & 角蟾科 & Megophryidae & 雷山髭蟾 & Vibrissaphora leishanense & y & \\
\hline 73 & 两栖纲 & Amphibia & 无尾目 & Anura & 角蟾科 & Megophryidae & 崇安髭蟾 & Vibrissaphora liui & si & \\
\hline 74 & 两栖纲 & Amphibia & 无尾目 & Anura & 角蟾科 & Megophryidae & 高山掌突蟾 & Paramegophrys alpinus & hc & \\
\hline 75 & 两栖纲 & Amphibia & 无尾目 & Anura & 角蟾科 & Megophryidae & 福建掌突蟾 & Paramegophrys liui & $\mathrm{sb}$ & \\
\hline 76 & 两栖纲 & Amphibia & 无尾目 & Anura & 角蟾科 & Megophryidae & 峨山掌突蟾 & Paramegophrys oshanensis & hc & \\
\hline 77 & 两栖纲 & Amphibia & 无尾目 & Anura & 角蟾科 & Megophryidae & 蟼掌突蟾 & Paramegophrys pelodytoides & wd & \\
\hline 78 & 两栖纲 & Amphibia & 无尾目 & Anura & 角蟾科 & Megophryidae & 腹斑掌突蟾 & Paramegophrys ventripunctatus & sa & \\
\hline 79 & 两栖纲 & Amphibia & 无尾目 & Anura & 角蟾科 & Megophryidae & 宽头短腿蟾 & Brachytarsophrys carinensis & $\mathrm{sc}$ & \\
\hline 80 & 两栖纲 & Amphibia & 无尾目 & Anura & 角蟾科 & Megophryidae & 大花角蟾 & Megophrys gigantica & hc & \\
\hline 81 & 两栖纲 & Amphibia & 无尾目 & Anura & 角蟾科 & Megophryidae & 南江角蟾 & Megophrys nankiangensis & si & \\
\hline 82 & 两栖纲 & Amphibia & 无尾目 & Anura & 角蟾科 & Megophryidae & 沙坪角蟾 & Megophrys shapingensis & hc & \\
\hline 83 & 两栖纲 & Amphibia & 无尾目 & Anura & 角蟾科 & Megophryidae & 腺角蟾 & Megophrys glandulosa & hc & \\
\hline 84 & 两栖纲 & Amphibia & 无尾目 & Anura & 角蟾科 & Megophryidae & 大角蟾 & Megophrys major & wb & \\
\hline 85 & 两栖纲 & Amphibia & 无尾目 & Anura & 角蟾科 & Megophryidae & 莽山角蟾 & Megophrys mangshanensis & si & \\
\hline 86 & 两栖纲 & Amphibia & 无尾目 & Anura & 角蟾科 & Megophryidae & 墨脱角蟾 & Megophrys medogensis & he & \\
\hline
\end{tabular}


郜二虎, 何杰坤, 王志臣, 徐扬, 唐小平, 江海声. 全国陆生野生动物调查单元区划方案. 生物多样性, 2017, 25 (12): 1321-1330.

http://www.biodiversity-science.net/CN/10.17520/biods.2017135

\begin{tabular}{|c|c|c|c|c|c|c|c|c|c|c|}
\hline \multirow{2}{*}{$\begin{array}{l}\text { 序号 } \\
\text { No. } \\
87\end{array}$} & \multicolumn{2}{|c|}{$\begin{array}{c}\text { 纲 } \\
\text { Class }\end{array}$} & \multicolumn{2}{|c|}{$\begin{array}{c}\text { 目 } \\
\text { Order }\end{array}$} & \multicolumn{2}{|r|}{$\begin{array}{c}\text { 科 } \\
\text { Family }\end{array}$} & \multicolumn{2}{|r|}{$\begin{array}{l}\text { 物种名 } \\
\text { Species }\end{array}$} & \multirow{2}{*}{$\begin{array}{l}\text { 分布型 } \\
\text { Chorotype } \\
\text { hc }\end{array}$} & \multirow[t]{2}{*}{$\begin{array}{c}\text { 备注 } \\
\text { Remarks }\end{array}$} \\
\hline & 两栖纲 & Amphibia & 无尾目 & Anura & 角蟾科 & Megophryidae & 峨眉角蟾 & Megophrys omeimontis & & \\
\hline 88 & 两栖纲 & Amphibia & 无尾目 & Anura & 角蟾科 & Megophryidae & 棘指角蟾 & Megophrys spinata & y & \\
\hline 89 & 两栖纲 & Amphibia & 无尾目 & Anura & 角蛤科 & Megophryidae & 淡肩角蟾 & Megophrys boettgeri & sd & \\
\hline 90 & 两栖纲 & Amphibia & 无尾目 & Anura & 角蛿科 & Megophryidae & 挂墩角蟾 & Megophrys kuatunensis & si & \\
\hline 91 & 两栖纲 & Amphibia & 无尾目 & Anura & 角蟾科 & Megophryidae & 短肢角蟾 & Megophrys brachykolos & $\mathrm{sb}$ & \\
\hline 92 & 两栖纲 & Amphibia & 无尾目 & Anura & 角蛤科 & Megophryidae & 小角蟾 & Megophrys minor & sd & \\
\hline 93 & 两栖纲 & Amphibia & 无尾目 & Anura & 角蟾科 & Megophryidae & 无量山角蟾 & Megophrys wuliangshanensis & hc & \\
\hline 94 & 两栖纲 & Amphibia & 无尾目 & Anura & 角蟾科 & Megophryidae & 巫山角蟾 & Megophrys wushanensis & sn & \\
\hline 95 & 两栖纲 & Amphibia & 无尾目 & Anura & 角蛿科 & Megophryidae & 凸肛角蟾 & Megophrys pachyproctus & si & \\
\hline 96 & 两栖纲 & Amphibia & 无尾目 & Anura & 角蟾科 & Megophryidae & 粗皮角蟾 & Megophrys palpebralespinosa & sa & \\
\hline 97 & 两栖纲 & Amphibia & 无尾目 & Anura & 角蟾科 & Megophryidae & 凹顶角蟾 & Megophrys parva & wa & \\
\hline 98 & 两栖纲 & Amphibia & 无尾目 & Anura & 角蟾科 & Megophryidae & 张氏角蟾 & Megophrys zhangi & ha & \\
\hline 99 & 两栖纲 & Amphibia & 无尾目 & Anura & 角蟾科 & Megophryidae & 小口拟角蟾 & Ophryophryne microstoma & sd & \\
\hline 100 & 两栖纲 & Amphibia & 无尾目 & Anura & 角蛿科 & Megophryidae & 突肛拟角蟾 & Ophryophryne pachyproctus & sa & \\
\hline 101 & 两栖纲 & Amphibia & 无尾目 & Anura & 蟾蜍科 & Bufonidae & 哀牢蟾蜍 & Bufo ailaoanus & hc & \\
\hline 102 & 两栖纲 & Amphibia & 无尾目 & Anura & 蟾蜍科 & Bufonidae & 隐耳蟾蜍 & Bufo cryptotympanicus & sc & \\
\hline 103 & 两栖纲 & Amphibia & 无尾目 & Anura & 蟾蜍科 & Bufonidae & 史氏蟾蜍 & Bufo stejnegeri & $\mathrm{kd}$ & \\
\hline 104 & 两栖纲 & Amphibia & 无尾目 & Anura & 蟾蜍科 & Bufonidae & 喜山蟾蜍 & Bufo himalayanus & ha & \\
\hline 105 & 两栖纲 & Amphibia & 无尾目 & Anura & 蟾蜍科 & Bufonidae & 黑眶蟾蜍 & Bufo melanostictus & wc & \\
\hline 106 & 两栖纲 & Amphibia & 无尾目 & Anura & 蟾蜍科 & Bufonidae & 中华蟾蜍 & Bufo gargarizans & eg & \\
\hline 107 & 两栖纲 & Amphibia & 无尾目 & Anura & 蟾蜍科 & Bufonidae & 西藏蟾蜍 & Bufo tibetanus & hc & \\
\hline 108 & 两栖纲 & Amphibia & 无尾目 & Anura & 蟾蜍科 & Bufonidae & 圆病蟾蜍 & Bufo tuberculatus & he & \\
\hline
\end{tabular}


郜二虎, 何杰坤, 王志臣, 徐扬, 唐小平, 江海声. 全国陆生野生动物调查单元区划方案. 生物多样性, 2017, 25 (12): 1321-1330.

http://www.biodiversity-science.net/CN/10.17520/biods.2017135

\begin{tabular}{|c|c|c|c|c|c|c|c|c|c|c|}
\hline \multirow{2}{*}{$\begin{array}{l}\text { 序号 } \\
\text { No. } \\
109\end{array}$} & \multicolumn{2}{|c|}{$\begin{array}{c}\text { 纲 } \\
\text { Class }\end{array}$} & \multicolumn{2}{|c|}{$\begin{array}{c}\text { 目 } \\
\text { Order }\end{array}$} & \multicolumn{2}{|r|}{$\begin{array}{c}\text { 科 } \\
\text { Family }\end{array}$} & \multicolumn{2}{|r|}{$\begin{array}{l}\text { 物种名 } \\
\text { Species }\end{array}$} & \multirow{2}{*}{$\begin{array}{l}\begin{array}{c}\text { 分布型 } \\
\text { Chorotype }\end{array} \\
d\end{array}$} & \multirow[t]{2}{*}{$\begin{array}{c}\text { 备注 } \\
\text { Remarks }\end{array}$} \\
\hline & 两栖纲 & Amphibia & 无尾目 & Anura & 蟾蜍科 & Bufonidae & 塔里木蟾蜍 & Bufo pewzowi & & \\
\hline 110 & 两栖纲 & Amphibia & 无尾目 & Anura & 蟾蜍科 & Bufonidae & 花背蟾蜍 & Bufo raddei & $\mathrm{xg}$ & \\
\hline 111 & 两栖纲 & Amphibia & 无尾目 & Anura & 蟾蜍科 & Bufonidae & 无梀溪蟾 & Torrentophryne aspinia & sa & \\
\hline 112 & 两栖纲 & Amphibia & 无尾目 & Anura & 蟾蜍科 & Bufonidae & 缅甸溪蟾 & Torrentophryne burmanus & hc & \\
\hline 113 & 两栖纲 & Amphibia & 无尾目 & Anura & 蟾蜍科 & Bufonidae & 鳞皮小蟾 & Parapelophryne scalpta & $\mathrm{j}$ & \\
\hline 114 & 两栖纲 & Amphibia & 无尾目 & Anura & 雨蛙科 & Hylidae & 东北雨蛙 & Hyla ussuriensis & $\mathrm{ma}$ & \\
\hline 115 & 两栖纲 & Amphibia & 无尾目 & Anura & 雨蛙科 & Hylidae & 华南雨蛙 & Hyla simplex & wc & \\
\hline 116 & 两栖纲 & Amphibia & 无尾目 & Anura & 雨蛙科 & Hylidae & 昭平雨蛙 & Hyla zhaopingensis & si & \\
\hline 117 & 两栖纲 & Amphibia & 无尾目 & Anura & 雨蛙科 & Hylidae & 华西雨蛙 & Hyla gongshanensis & wd & \\
\hline 118 & 两栖纲 & Amphibia & 无尾目 & Anura & 雨蛙科 & Hylidae & 秦岭雨蛙 & Hyla tsinlingensis & 1 & \\
\hline 119 & 两栖纲 & Amphibia & 无尾目 & Anura & 雨蛙科 & Hylidae & 中国雨蛙 & Hyla chinensis & sd & \\
\hline 120 & 两栖纲 & Amphibia & 无尾目 & Anura & 雨蛙科 & Hylidae & 三港雨蛙 & Hyla sanchiangensis & si & \\
\hline 121 & 两栖纲 & Amphibia & 无尾目 & Anura & 树蛙科 & Rhacophoridae & 日本溪树蛙 & Buergeria japonica & $\mathrm{j}$ & \\
\hline 122 & 两栖纲 & Amphibia & 无尾目 & Anura & 树蛙科 & Rhacophoridae & 海南溪树蛙 & Buergeria oxycephala & $\mathrm{j}$ & \\
\hline 123 & 两栖纲 & Amphibia & 无尾目 & Anura & 树蛙科 & Rhacophoridae & 锯腿水树蛙 & Aquixalus odontotarsus & $\mathrm{s}$ & \\
\hline 124 & 两栖纲 & Amphibia & 无尾目 & Anura & 树蛙科 & Rhacophoridae & 金秀水树蛙 & Aquixalus jinxiuensis & si & \\
\hline 125 & 两栖纲 & Amphibia & 无尾目 & Anura & 树蛙科 & Rhacophoridae & 墨脱水树蛙 & Aquixalus medogensis & he & \\
\hline 126 & 两栖纲 & Amphibia & 无尾目 & Anura & 树蛙科 & Rhacophoridae & 黑眼睑水树蛙 & Aquixalus gracilipes & $\mathrm{sa}$ & \\
\hline 127 & 两栖纲 & Amphibia & 无尾目 & Anura & 树蛙科 & Rhacophoridae & 海南水树蛙 & Aquixalus hainanus & $\mathrm{j}$ & \\
\hline 128 & 两栖纲 & Amphibia & 无尾目 & Anura & 树蛙科 & Rhacophoridae & 眼斑水树蛙 & Aquixalus ocellatus & $\mathrm{j}$ & \\
\hline 129 & 两栖纲 & Amphibia & 无尾目 & Anura & 树蛙科 & Rhacophoridae & 白㚘水树蛙 & Aquixalus palpebralis & wa & \\
\hline 130 & 两栖纲 & Amphibia & 无尾目 & Anura & 树蛙科 & Rhacophoridae & 罗默水树蛙 & Aquixalus romeri & j & \\
\hline
\end{tabular}


郜二虎, 何杰坤, 王志臣, 徐扬, 唐小平, 江海声. 全国陆生野生动物调查单元区划方案. 生物多样性, 2017, 25 (12): 1321-1330.

http://www.biodiversity-science.net/CN/10.17520/biods.2017135

\begin{tabular}{|c|c|c|c|c|c|c|c|c|c|c|}
\hline \multirow{2}{*}{$\begin{array}{l}\text { 序号 } \\
\text { No. } \\
131\end{array}$} & \multicolumn{2}{|c|}{$\begin{array}{c}\text { 纲 } \\
\text { Class }\end{array}$} & \multicolumn{2}{|c|}{$\begin{array}{c}\text { 目 } \\
\text { Order }\end{array}$} & \multicolumn{2}{|r|}{$\begin{array}{c}\text { 科 } \\
\text { Family }\end{array}$} & \multicolumn{2}{|r|}{$\begin{array}{l}\text { 物种名 } \\
\text { Species }\end{array}$} & \multirow{2}{*}{$\begin{array}{l}\text { 分布型 } \\
\text { Chorotype } \\
\text { si }\end{array}$} & \multirow[t]{2}{*}{$\begin{array}{c}\text { 备注 } \\
\text { Remarks }\end{array}$} \\
\hline & 两栖纲 & Amphibia & 无尾目 & Anura & 树蛙科 & Rhacophoridae & 白斑水树蛙 & Aquixalus albopunctatus & & \\
\hline 132 & 两栖纲 & Amphibia & 无尾目 & Anura & 树蛙科 & Rhacophoridae & 粗皮水树蛙 & Aquixalus asper & wa & \\
\hline 133 & 两栖纲 & Amphibia & 无尾目 & Anura & 树蛙科 & Rhacophoridae & 疮水树蛙 & Aquixalus tuberculatus & he & \\
\hline 134 & 两栖纲 & Amphibia & 无尾目 & Anura & 树蛙科 & Rhacophoridae & 陇川水树蛙 & Aquixalus longchuanensis & $\mathrm{sa}$ & \\
\hline 135 & 两栖纲 & Amphibia & 无尾目 & Anura & 树蛙科 & Rhacophoridae & 预腊水树蛙 & Aquixalus menglaensis & sa & \\
\hline 136 & 两栖纲 & Amphibia & 无尾目 & Anura & 树蛙科 & Rhacophoridae & 红吸盘水树蛙 & Aquixalus rhododiscus & $\mathrm{se}$ & \\
\hline 137 & 两栖纲 & Amphibia & 无尾目 & Anura & 树蛙科 & Rhacophoridae & 背条跳树蛙 & Chirixalus doriae & $\mathrm{wb}$ & \\
\hline 138 & 两栖纲 & Amphibia & 无尾目 & Anura & 树蛙科 & Rhacophoridae & 侧条跳树蛙 & Chirixalus vittatus & wc & \\
\hline 139 & 两栖纲 & Amphibia & 无尾目 & Anura & 树蛙科 & Rhacophoridae & 广西棱皮树蛙 & Theloderma kwangsiensis & sg & \\
\hline 140 & 两栖纲 & Amphibia & 无尾目 & Anura & 树蛙科 & Rhacophoridae & 斑腿泛树蛙 & Polypedates megacephalus & wd & \\
\hline 141 & 两栖纲 & Amphibia & 无尾目 & Anura & 树蛙科 & Rhacophoridae & 无声囊泛树蛙 & Polypedates mutus & $\mathrm{sc}$ & \\
\hline 142 & 两栖纲 & Amphibia & 无尾目 & Anura & 树蛙科 & Rhacophoridae & 双斑树蛙 & Rhacophorus bipunctatus & wa & \\
\hline 143 & 两栖纲 & Amphibia & 无尾目 & Anura & 树蛙科 & Rhacophoridae & 黑蹼树蛙 & Rhacophorus kio & wa & \\
\hline 144 & 两栖纲 & Amphibia & 无尾目 & Anura & 树蛙科 & Rhacophoridae & 红蹼树蛙 & Rhacophorus rhodopus & $\mathrm{sb}$ & \\
\hline 145 & 两栖纲 & Amphibia & 无尾目 & Anura & 树蛙科 & Rhacophoridae & 横纹树蛙 & Rhacophorus translineatus & he & \\
\hline 146 & 两栖纲 & Amphibia & 无尾目 & Anura & 树蛙科 & Rhacophoridae & 疮足树蛙 & Rhacophorus verrucopus & he & \\
\hline 147 & 两栖纲 & Amphibia & 无尾目 & Anura & 树蛙科 & Rhacophoridae & 大树蛙 & Rhacophorus dennysi & $\mathrm{sc}$ & \\
\hline 148 & 两栖纲 & Amphibia & 无尾目 & Anura & 树蛙科 & Rhacophoridae & 棕褶树蛙 & Rhacophorus feae & wa & \\
\hline 149 & 两栖纲 & Amphibia & 无尾目 & Anura & 树蛙科 & Rhacophoridae & 白领大树蛙 & Rhacophorus maximus & wa & \\
\hline 150 & 两栖纲 & Amphibia & 无尾目 & Anura & 树蛙科 & Rhacophoridae & 贡山树蛙 & Rhacophorus gongshanensis & he & \\
\hline 151 & 两栖纲 & Amphibia & 无尾目 & Anura & 树蛙科 & Rhacophoridae & 峨眉树蛙 & Rhacophorus omeimontis & hc & \\
\hline 152 & 两栖纲 & Amphibia & 无尾目 & Anura & 树蛙科 & Rhacophoridae & 诸罗树蛙 & Rhacophorus arvalis & j & \\
\hline
\end{tabular}


郜二虎, 何杰坤, 王志臣, 徐扬, 唐小平, 江海声. 全国陆生野生动物调查单元区划方案. 生物多样性, 2017, 25 (12): 1321-1330.

http://www.biodiversity-science.net/CN/10.17520/biods.2017135

\begin{tabular}{|c|c|c|c|c|c|c|c|c|c|c|}
\hline \multirow{2}{*}{$\begin{array}{l}\text { 序号 } \\
\text { No. } \\
153\end{array}$} & \multicolumn{2}{|c|}{$\begin{array}{c}\text { 纲 } \\
\text { Class }\end{array}$} & \multicolumn{2}{|c|}{$\begin{array}{c}\text { 目 } \\
\text { Order }\end{array}$} & \multicolumn{2}{|r|}{$\begin{array}{c}\text { 科 } \\
\text { Family }\end{array}$} & \multicolumn{2}{|r|}{$\begin{array}{l}\text { 物种名 } \\
\text { Species }\end{array}$} & \multirow{2}{*}{$\begin{array}{l}\begin{array}{c}\text { 分布型 } \\
\text { Chorotype }\end{array} \\
\mathrm{j}\end{array}$} & \multirow[t]{2}{*}{$\begin{array}{c}\text { 备注 } \\
\text { Remarks }\end{array}$} \\
\hline & 两栖纲 & Amphibia & 无尾目 & Anura & 树蛙科 & Rhacophoridae & 橙腹树蛙 & Rhacophorus aurantiventris & & \\
\hline 154 & 两栖纲 & Amphibia & 无尾目 & Anura & 树蛙科 & Rhacophoridae & 翡翠树蛙 & Rhacophorus prasinatus & $\mathrm{j}$ & \\
\hline 155 & 两栖纲 & Amphibia & 无尾目 & Anura & 树蛙科 & Rhacophoridae & 台北树蛙 & Rhacophorus taipeianus & $\mathrm{j}$ & \\
\hline 156 & 两栖纲 & Amphibia & 无尾目 & Anura & 树蛙科 & Rhacophoridae & 经甫树蛙 & Rhacophorus chenfui & si & \\
\hline 157 & 两栖纲 & Amphibia & 无尾目 & Anura & 树蛙科 & Rhacophoridae & 宝兴树蛙 & Rhacophorus dugritei & hc & \\
\hline 158 & 两栖纲 & Amphibia & 无尾目 & Anura & 树蛙科 & Rhacophoridae & 洪佛树蛙 & Rhacophorus hungfuensis & si & \\
\hline 159 & 两栖纲 & Amphibia & 无尾目 & Anura & 树蛙科 & Rhacophoridae & 黑点树蛙 & Rhacophorus nigropunctatus & sc & \\
\hline 160 & 两栖纲 & Amphibia & 无尾目 & Anura & 树蛙科 & Rhacophoridae & 瑶山树蛙 & Rhacophorus yaoshanensis & sg & \\
\hline 161 & 两栖纲 & Amphibia & 无尾目 & Anura & 姬蛙科 & Microhylidae & 云南小狭口蛙 & Calluella yunnanensis & $\mathrm{y}$ & \\
\hline 162 & 两栖纲 & Amphibia & 无尾目 & Anura & 姬蛙科 & Microhylidae & 粗皮姬蛙 & Microhyla butleri & wc & \\
\hline 163 & 两栖纲 & Amphibia & 无尾目 & Anura & 姬蛙科 & Microhylidae & 大姬蛙 & Microhyla fowleri & wa & \\
\hline 164 & 两栖纲 & Amphibia & 无尾目 & Anura & 姬蛙科 & Microhylidae & 小弧斑姬蛙 & Microhyla heymonsi & wc & \\
\hline 165 & 两栖纲 & Amphibia & 无尾目 & Anura & 姬蛙科 & Microhylidae & 合征姬蛙 & Microhyla mixtura & si & \\
\hline 166 & 两栖纲 & Amphibia & 无尾目 & Anura & 姬蛙科 & Microhylidae & 饰纹姬蛙 & Microhyla ornata & wc & \\
\hline 167 & 两栖纲 & Amphibia & 无尾目 & Anura & 姬蛙科 & Microhylidae & 花姬蛙 & Microhyla pulchra & wc & \\
\hline 168 & 两栖纲 & Amphibia & 无尾目 & Anura & 姬蛙科 & Microhylidae & 德力小姬蛙 & Micryletta inornata & wa & \\
\hline 169 & 两栖纲 & Amphibia & 无尾目 & Anura & 姬蛙科 & Microhylidae & 史氏小姬蛙 & Micryletta steinegeri & $\mathrm{j}$ & \\
\hline 170 & 两栖纲 & Amphibia & 无尾目 & Anura & 姬蛙科 & Microhylidae & 花狭口蛙 & Kaloula pulchra & $\mathrm{wb}$ & \\
\hline 171 & 两栖纲 & Amphibia & 无尾目 & Anura & 姬蛙科 & Microhylidae & 北方狭口蛙 & Kaloula borealis & $\mathrm{xb}$ & \\
\hline 172 & 两栖纲 & Amphibia & 无尾目 & Anura & 姬蛙科 & Microhylidae & 四川狭口蛙 & Kaloula rugifera & hc & \\
\hline 173 & 两栖纲 & Amphibia & 无尾目 & Anura & 姬蛙科 & Microhylidae & 多疮狭口蛙 & Kaloula verrucosa & hc & \\
\hline 174 & 两栖纲 & Amphibia & 无尾目 & Anura & 姬蛙科 & Microhylidae & 孟连细狭口蛙 & Kalophrynus menglienicus & sa & \\
\hline
\end{tabular}


郜二虎, 何杰坤, 王志臣, 徐扬, 唐小平, 江海声. 全国陆生野生动物调查单元区划方案. 生物多样性, 2017, 25 (12): 1321-1330.

http://www.biodiversity-science.net/CN/10.17520/biods.2017135

\begin{tabular}{|c|c|c|c|c|c|c|c|c|c|c|}
\hline \multirow{2}{*}{$\begin{array}{l}\text { 序号 } \\
\text { No. } \\
175\end{array}$} & \multicolumn{2}{|c|}{$\begin{array}{c}\text { 纲 } \\
\text { Class }\end{array}$} & \multicolumn{2}{|c|}{$\begin{array}{c}\text { 目 } \\
\text { Order }\end{array}$} & \multicolumn{2}{|r|}{$\begin{array}{c}\text { 科 } \\
\text { Family }\end{array}$} & \multicolumn{2}{|r|}{$\begin{array}{l}\text { 物种名 } \\
\text { Species }\end{array}$} & \multirow{2}{*}{$\begin{array}{l}\text { 分布型 } \\
\text { Chorotype } \\
\text { wb }\end{array}$} & \multirow[t]{2}{*}{$\begin{array}{c}\text { 备注 } \\
\text { Remarks }\end{array}$} \\
\hline & 两栖纲 & Amphibia & 无尾目 & Anura & 姬蛙科 & Microhylidae & 花细狭口蛙 & Kalophrynus interlineatus & & \\
\hline 176 & 两栖纲 & Amphibia & 无尾目 & Anura & 蛙科 & Ranidae & 昭觉林蛙 & Rana chaochiaoensis & he & \\
\hline 177 & 两栖纲 & Amphibia & 无尾目 & Anura & 蛙科 & Ranidae & 峰斑林蛙 & Rana chevronta & hc & \\
\hline 178 & 两栖纲 & Amphibia & 无尾目 & Anura & 蛙科 & Ranidae & 长肢林蛙 & Rana longicrus & $\mathrm{sb}$ & \\
\hline 179 & 两栖纲 & Amphibia & 无尾目 & Anura & 蛙科 & Ranidae & 峨眉林蛙 & Rana omeimontis & sh & \\
\hline 180 & 两栖纲 & Amphibia & 无尾目 & Anura & 蛙科 & Ranidae & 镇海林蛙 & Rana zhenhaiensis & sd & \\
\hline 181 & 两栖纲 & Amphibia & 无尾目 & Anura & 蛙科 & Ranidae & 中亚林蛙 & Rana asiatica & d & \\
\hline 182 & 两栖纲 & Amphibia & 无尾目 & Anura & 蛙科 & Ranidae & 中国林蛙 & Rana chensinensis & xa & \\
\hline 183 & 两栖纲 & Amphibia & 无尾目 & Anura & 蛙科 & Ranidae & 阿尔泰林蛙 & Rana altaica & d & \\
\hline 184 & 两栖纲 & Amphibia & 无尾目 & Anura & 蛙科 & Ranidae & 黑龙江林蛙 & Rana amurensis & ub & \\
\hline 185 & 两栖纲 & Amphibia & 无尾目 & Anura & 蛙科 & Ranidae & 桓仁林蛙 & Rana huanrenensis & $\mathrm{k}$ & \\
\hline 186 & 两栖纲 & Amphibia & 无尾目 & Anura & 蛙科 & Ranidae & 湖北侧褶蛙 & Pelophylax hubeiensis & si & \\
\hline 187 & 两栖纲 & Amphibia & 无尾目 & Anura & 蛙科 & Ranidae & 金线侧褶蛙 & Pelophylax plancyi & ea & \\
\hline 188 & 两栖纲 & Amphibia & 无尾目 & Anura & 蛙科 & Ranidae & 黑斑侧褶蛙 & Pelophylax nigromaculatus & ea & \\
\hline 189 & 两栖纲 & Amphibia & 无尾目 & Anura & 蛙科 & Ranidae & 中亚侧褶蛙 & Pelophylax terentievi & d & \\
\hline 190 & 两栖纲 & Amphibia & 无尾目 & Anura & 蛙科 & Ranidae & 黑斜线侧褶蛙 & Pelophylax nigrolineatus & $\mathrm{sa}$ & \\
\hline 191 & 两栖纲 & Amphibia & 无尾目 & Anura & 蛙科 & Ranidae & 滇侧褶蛙 & Pelophylax pleuraden & $\mathrm{yb}$ & \\
\hline 192 & 两栖纲 & Amphibia & 无尾目 & Anura & 蛙科 & Ranidae & 胫腺侧褶蛙 & Pelophylax shuchinae & hc & \\
\hline 193 & 两栖纲 & Amphibia & 无尾目 & Anura & 蛙科 & Ranidae & 东北粗皮蛙 & Rugosa emeljanovi & $\mathrm{k}$ & \\
\hline 194 & 两栖纲 & Amphibia & 无尾目 & Anura & 蛙科 & Ranidae & 天台粗皮蛙 & Rugosa tientaiensis & si & \\
\hline 195 & 两栖纲 & Amphibia & 无尾目 & Anura & 蛙科 & Ranidae & 小腺蛙 & Glandirana minima & sg & \\
\hline 196 & 两栖纲 & Amphibia & 无尾目 & Anura & 蛙科 & Ranidae & 桑植趾沟蛙 & Pseudorana sangzhiensis & si & \\
\hline
\end{tabular}


郜二虎, 何杰坤, 王志臣, 徐扬, 唐小平, 江海声. 全国陆生野生动物调查单元区划方案. 生物多样性, 2017, 25 (12): 1321-1330.

http://www.biodiversity-science.net/CN/10.17520/biods.2017135

\begin{tabular}{|c|c|c|c|c|c|c|c|c|c|c|}
\hline \multirow{2}{*}{$\begin{array}{l}\text { 序号 } \\
\text { No. } \\
197\end{array}$} & \multicolumn{2}{|c|}{$\begin{array}{c}\text { 纲 } \\
\text { Class }\end{array}$} & \multicolumn{2}{|c|}{$\begin{array}{c}\text { 目 } \\
\text { Order }\end{array}$} & \multicolumn{2}{|r|}{$\begin{array}{c}\text { 科 } \\
\text { Family }\end{array}$} & \multicolumn{2}{|r|}{$\begin{array}{l}\text { 物种名 } \\
\text { Species }\end{array}$} & \multirow{2}{*}{$\begin{array}{l}\begin{array}{c}\text { 分布型 } \\
\text { Chorotype }\end{array} \\
\text { ya }\end{array}$} & \multirow[t]{2}{*}{$\begin{array}{c}\text { 备注 } \\
\text { Remarks }\end{array}$} \\
\hline & 两栖纲 & Amphibia & 无尾目 & Anura & 蛙科 & Ranidae & 威宁趾沟蛙 & Pseudorana weiningensis & & \\
\hline 198 & 两栖纲 & Amphibia & 无尾目 & Anura & 蛙科 & Ranidae & 黑耳水蛙 & Hylarana nigrotympanica & wb & \\
\hline 199 & 两栖纲 & Amphibia & 无尾目 & Anura & 蛙科 & Ranidae & 沼水蛙 & Hylarana guentheri & sc & \\
\hline 200 & 两栖纲 & Amphibia & 无尾目 & Anura & 蛙科 & Ranidae & 阔褶水蛙 & Hylarana latouchii & se & \\
\hline 201 & 两栖纲 & Amphibia & 无尾目 & Anura & 蛙科 & Ranidae & 细刺水蛙 & Hylarana spinulosa & $\mathrm{j}$ & \\
\hline 202 & 两栖纲 & Amphibia & 无尾目 & Anura & 蛙科 & Ranidae & 仙琴蛙 & Hylarana daunchina & hc & \\
\hline 203 & 两栖纲 & Amphibia & 无尾目 & Anura & 蛙科 & Ranidae & 弹琴蛙 & Hylarana adenopleura & sc & \\
\hline 204 & 两栖纲 & Amphibia & 无尾目 & Anura & 蛙科 & Ranidae & 长趾纤蛙 & Hylarana macrodactyla & wb & \\
\hline 205 & 两栖纲 & Amphibia & 无尾目 & Anura & 蛙科 & Ranidae & 台北纤蛙 & Hylarana taipehensis & wb & \\
\hline 206 & 两栖纲 & Amphibia & 无尾目 & Anura & 蛙科 & Ranidae & 竹叶蛙 & Odorrana versabilis & si & \\
\hline 207 & 两栖纲 & Amphibia & 无尾目 & Anura & 蛙科 & Ranidae & 凹耳臭蛙 & Odorrana tormota & si & \\
\hline 208 & 两栖纲 & Amphibia & 无尾目 & Anura & 蛙科 & Ranidae & 台岛臭蛙 & Odorrana taiwaniana & $\mathrm{j}$ & \\
\hline 209 & 两栖纲 & Amphibia & 无尾目 & Anura & 蛙科 & Ranidae & 光雾臭蛙 & Odorrana kuangwuensis & si & \\
\hline 210 & 两栖纲 & Amphibia & 无尾目 & Anura & 蛙科 & Ranidae & 绿臭蛙 & Odorrana margaretae & sh & \\
\hline 211 & 两栖纲 & Amphibia & 无尾目 & Anura & 蛙科 & Ranidae & 务川臭蛙 & Odorrana wuchuanensis & si & \\
\hline 212 & 两栖纲 & Amphibia & 无尾目 & Anura & 蛙科 & Ranidae & 云南臭蛙 ～ & Odorrana andersonii & wc & \\
\hline 213 & 两栖纲 & Amphibia & 无尾目 & Anura & 蛙科 & Ranidae & 无指盘臭蛙 & Odorrana grahami & hc & \\
\hline 214 & 两栖纲 & Amphibia & 无尾目 & Anura & 蛙科 & Ranidae & 安龙臭蛙 & Odorrana anlungensis & sg & \\
\hline 215 & 两栖纲 & Amphibia & 无尾目 & Anura & 蛙科 & Ranidae & 龙胜臭蛙 & Odorrana lungshengensis & si & \\
\hline 216 & 两栖纲 & Amphibia & 无尾目 & Anura & 蛙科 & Ranidae & 花臭蛙 & Odorrana schmackeri & si & \\
\hline 217 & 两栖纲 & Amphibia & 无尾目 & Anura & 蛙科 & Ranidae & 滇南臭蛙 & Odorrana tiannanensis & sa & \\
\hline 218 & 两栖纲 & Amphibia & 无尾目 & Anura & 蛙科 & Ranidae & 海陆蛙 & Fejervarya cancrivora & wa & \\
\hline
\end{tabular}


郜二虎, 何杰坤, 王志臣, 徐扬, 唐小平, 江海声. 全国陆生野生动物调查单元区划方案. 生物多样性, 2017, 25 (12): 1321-1330.

http://www.biodiversity-science.net/CN/10.17520/biods.2017135

\begin{tabular}{|c|c|c|c|c|c|c|c|c|c|c|}
\hline $\begin{array}{r}\text { 序号 } \\
\text { No. }\end{array}$ & \multicolumn{2}{|c|}{$\begin{array}{c}\text { 纲 } \\
\text { Class }\end{array}$} & \multicolumn{2}{|c|}{$\begin{array}{c}\text { 目 } \\
\text { Order }\end{array}$} & \multicolumn{2}{|r|}{$\begin{array}{c}\text { 科 } \\
\text { Family }\end{array}$} & \multicolumn{2}{|r|}{$\begin{array}{l}\text { 物种名 } \\
\text { Species }\end{array}$} & \multirow{2}{*}{$\begin{array}{l}\begin{array}{c}\text { 分布型 } \\
\text { Chorotype }\end{array} \\
\text { we }\end{array}$} & \multirow[t]{2}{*}{$\begin{array}{c}\text { 备注 } \\
\text { Remarks }\end{array}$} \\
\hline 219 & 两栖纲 & Amphibia & 无尾目 & Anura & 蛙科 & Ranidae & 泽陆蛙 & Fejervarya multistriata & & \\
\hline 220 & 两栖纲 & Amphibia & 无尾目 & Anura & 蛙科 & Ranidae & 虎纹蛙 & Hoplobatrachus chinenses & wc & \\
\hline 221 & 两栖纲 & Amphibia & 无尾目 & Anura & 蛙科 & Ranidae & 版纳大头蛙 & Limnonectes bannaensis & wa & \\
\hline 222 & 两栖纲 & Amphibia & 无尾目 & Anura & 蛙科 & Ranidae & 脆皮大头蛙 & Limnonectes fragilis & $\mathrm{j}$ & \\
\hline 223 & 两栖纲 & Amphibia & 无尾目 & Anura & 蛙科 & Ranidae & 棘腹蛙 & Paa boulengeri & ha & \\
\hline 224 & 两栖纲 & Amphibia & 无尾目 & Anura & 蛙科 & Ranidae & 合江棘蛙 & Paa robertingeri & si & \\
\hline 225 & 两栖纲 & Amphibia & 无尾目 & Anura & 蛙科 & Ranidae & 棘侧蛙 & Paa shini & $\mathrm{y}$ & \\
\hline 226 & 两栖纲 & Amphibia & 无尾目 & Anura & 蛙科 & Ranidae & 小棘蛙 & Paa exilispinosa & $\mathrm{sc}$ & \\
\hline 227 & 两栖纲 & Amphibia & 无尾目 & Anura & 蛙科 & Ranidae & 九龙棘蛙 & Paa jiulongensis & si & \\
\hline 228 & 两栖纲 & Amphibia & 无尾目 & Anura & 蛙科 & Ranidae & 棘胸蛙 & Paa spinosa & $\mathrm{sc}$ & \\
\hline 229 & 两栖纲 & Amphibia & 无尾目 & Anura & 蛙科 & Ranidae & 棘臂蛙 & Paa liebigii & ha & \\
\hline 230 & 两栖纲 & Amphibia & 无尾目 & Anura & 蛙科 & Ranidae & 布兰福棘蛙 & Paa blanfordii & ha & \\
\hline 231 & 两栖纲 & Amphibia & 无尾目 & Anura & 蛙科 & Ranidae & 波留宁棘蛙 & Paa polunini & ha & \\
\hline 232 & 两栖纲 & Amphibia & 无尾目 & Anura & 蛙科 & Ranidae & 花棘蛙 & Paa maculosa & hc & \\
\hline 233 & 两栖纲 & Amphibia & 无尾目 & Anura & 蛙科 & Ranidae & 无声囊棘蛙 & Paa liui & hc & \\
\hline 234 & 两栖纲 & Amphibia & 无尾目 & Anura & 蛙科 & Ranidae & 双团棘胸蛙 & Paa yunnanensis & hc & \\
\hline 235 & 两栖纲 & Amphibia & 无尾目 & Anura & 蛙科 & Ranidae & 隆肛蛙 & Feirana quadranus & sh & \\
\hline 236 & 两栖纲 & Amphibia & 无尾目 & Anura & 蛙科 & Ranidae & 棘肛蛙 & Unculuana unculuanus & hc & \\
\hline 237 & 两栖纲 & Amphibia & 无尾目 & Anura & 蛙科 & Ranidae & 高山倭蛙 & Nanorana parkeri & $\mathrm{pd}$ & \\
\hline 238 & 两栖纲 & Amphibia & 无尾目 & Anura & 蛙科 & Ranidae & 倭蛙 & Nanorana pleskei & $\mathrm{pc}$ & \\
\hline 239 & 两栖纲 & Amphibia & 无尾目 & Anura & 蛙科 & Ranidae & 腹斑倭蛙 & Nanorana ventripunctata & hc & \\
\hline 240 & 两栖纲 & Amphibia & 无尾目 & Anura & 蛙科 & Ranidae & 刘氏舌突蛙 & Liurana liui & hc & \\
\hline
\end{tabular}


郜二虎, 何杰坤, 王志臣, 徐扬, 唐小平, 江海声. 全国陆生野生动物调查单元区划方案. 生物多样性, 2017, 25 (12): 1321-1330.

http://www.biodiversity-science.net/CN/10.17520/biods.2017135

\begin{tabular}{|c|c|c|c|c|c|c|c|c|c|c|}
\hline $\begin{array}{r}\text { 序号 } \\
\text { No. }\end{array}$ & \multicolumn{2}{|c|}{$\begin{array}{c}\text { 纲 } \\
\text { Class }\end{array}$} & \multicolumn{2}{|c|}{$\begin{array}{c}\text { 目 } \\
\text { Order }\end{array}$} & \multicolumn{2}{|r|}{$\begin{array}{c}\text { 科 } \\
\text { Family }\end{array}$} & \multicolumn{2}{|r|}{$\begin{array}{l}\text { 物种名 } \\
\text { Species }\end{array}$} & \multirow{2}{*}{$\begin{array}{l}\begin{array}{c}\text { 分布型 } \\
\text { Chorotype }\end{array} \\
\text { he }\end{array}$} & \multirow[t]{2}{*}{$\begin{array}{c}\text { 备注 } \\
\text { Remarks }\end{array}$} \\
\hline 241 & 两栖纲 & Amphibia & 无尾目 & Anura & 蛙科 & Ranidae & 网纹舌突蛙 & Liurana reticulata & & \\
\hline 242 & 两栖纲 & Amphibia & 无尾目 & Anura & 蛙科 & Ranidae & 西藏舌突蛙 & Liurana xizangensis & he & \\
\hline 243 & 两栖纲 & Amphibia & 无尾目 & Anura & 蛙科 & Ranidae & 台湾拟湍蛙 & Pseudoamolops sauteri & wc & \\
\hline 244 & 两栖纲 & Amphibia & 无尾目 & Anura & 蛙科 & Ranidae & 崇安湍蛙 & Amolops chunganensis & $\mathrm{si}$ & \\
\hline 245 & 两栖纲 & Amphibia & 无尾目 & Anura & 蛙科 & Ranidae & 小耳湍蛙 & Amolops gerbillus & ha & \\
\hline 246 & 两栖纲 & Amphibia & 无尾目 & Anura & 蛙科 & Ranidae & 山湍蛙 & Amolops monticola & ha & \\
\hline 247 & 两栖纲 & Amphibia & 无尾目 & Anura & 蛙科 & Ranidae & 棘皮湍蛙 & Amolops granulosus & hc & \\
\hline 248 & 两栖纲 & Amphibia & 无尾目 & Anura & 蛙科 & Ranidae & 理县湍蛙 & Amolops lifanensis & hc & \\
\hline 249 & 两栖纲 & Amphibia & 无尾目 & Anura & 蛙科 & Ranidae & 棕点湍蛙 & Amolops loloensis & hc & \\
\hline 250 & 两栖纲 & Amphibia & 无尾目 & Anura & 蛙科 & Ranidae & 四川湍蛙 & Amolops mantzorum & $\mathrm{hc}$ & \\
\hline 251 & 两栖纲 & Amphibia & 无尾目 & Anura & 蛙科 & Ranidae & 绿点湍蛙 & Amolops viridimaculatus & hc & \\
\hline 252 & 两栖纲 & Amphibia & 无尾目 & Anura & 蛙科 & Ranidae & 西域湍蛙 & Amolops marmoratus & ha & \\
\hline 253 & 两栖纲 & Amphibia & 无尾目 & Anura & 蛙科 & Ranidae & 沙巴湍蛙 & Amolops chapaensis & sa & \\
\hline 254 & 两栖纲 & Amphibia & 无尾目 & Anura & 蛙科 & Ranidae & 戴云湍蛙 & Amolops daiyunensis & $\mathrm{sg}$ & \\
\hline 255 & 两栖纲 & Amphibia & 无尾目 & Anura & 蛙科 & Ranidae & 香港湍蛙 & Amolops hongkongensis & $\mathrm{j}$ & \\
\hline 256 & 两栖纲 & Amphibia & 无尾目 & Anura & 蛙科 & Ranidae & 华南湍蛙 & Amolops ricketti & $\mathrm{sc}$ & \\
\hline 257 & 两栖纲 & Amphibia & 无尾目 & Anura & 蛙科 & Ranidae & 武夷湍蛙 & Amolops wuyiensis & si & \\
\hline 258 & 两栖纲 & Amphibia & 无尾目 & Anura & 蛙科 & Ranidae & 海南湍蛙 & Amolops hainanensis & $\mathrm{j}$ & \\
\hline 259 & 两栖纲 & Amphibia & 无尾目 & Anura & 蛙科 & Ranidae & 小湍蛙 & Amolops torrentis & $\mathrm{j}$ & \\
\hline 260 & 两栖纲 & Amphibia & 无尾目 & Anura & 蛙科 & Ranidae & 尖舌浮蛙 & Occidozyga lima & $\mathrm{wb}$ & \\
\hline 261 & 两栖纲 & Amphibia & 无尾目 & Anura & 蛙科 & Ranidae & 北蟾舌蛙 & Phrynoglossus borealis & he & \\
\hline 262 & 两栖纲 & Amphibia & 无尾目 & Anura & 蛙科 & Ranidae & 圆蟾舌蛙 & Phrynoglossus martensii & $\mathrm{wb}$ & \\
\hline
\end{tabular}


郜二虎, 何杰坤, 王志臣, 徐扬, 唐小平, 江海声. 全国陆生野生动物调查单元区划方案. 生物多样性, 2017, 25 (12): 1321-1330.

http://www.biodiversity-science.net/CN/10.17520/biods.2017135

\begin{tabular}{|c|c|c|c|c|c|c|c|c|c|c|}
\hline $\begin{array}{r}\text { 序号 } \\
\text { No. }\end{array}$ & \multicolumn{2}{|c|}{$\begin{array}{c}\text { 纲 } \\
\text { Class }\end{array}$} & \multicolumn{2}{|c|}{$\begin{array}{c}\text { 目 } \\
\text { Order }\end{array}$} & \multicolumn{2}{|r|}{$\begin{array}{c}\text { 科 } \\
\text { Family }\end{array}$} & \multicolumn{2}{|r|}{$\begin{array}{l}\text { 物种名 } \\
\text { Species }\end{array}$} & \multirow{2}{*}{$\begin{array}{l}\begin{array}{c}\text { 分布型 } \\
\text { Chorotype }\end{array} \\
\text { sd }\end{array}$} & \multirow[t]{2}{*}{$\begin{array}{c}\text { 备注 } \\
\text { Remarks }\end{array}$} \\
\hline 263 & 爬行纲 & Reptilia & 龟鳖目 & Testudoformes & 鳖科 & Trionychidae & 山瑞鳖 & Palea steindachneri & & \\
\hline 264 & 爬行纲 & Reptilia & 龟鳖目 & Testudoformes & 鳖科 & Trionychidae & 鼋 & Pelochelys bibroni & ea & \\
\hline 265 & 爬行纲 & Reptilia & 龟鳖目 & Testudoformes & 鳖科 & Trionychidae & 斑宒 & Pelochelys maculatus & si & 斯氏鳘的同物异名 \\
\hline 266 & 爬行纲 & Reptilia & 龟鳖目 & Testudoformes & 鳖科 & Trionychidae & 鳖 & Pelodiscus sinensis & ea & \\
\hline 267 & 爬行纲 & Reptilia & 龟鳖目 & Testudoformes & 鳖科 & Trionychidae & 斯氏鳖 & Rafetus swinhoei & $\mathrm{sc}$ & \\
\hline 268 & 爬行纲 & Reptilia & 龟鳖目 & Testudoformes & 平胸龟科 & Platysternidae & 平胸龟 & Platysternon megacephalum & wc & \\
\hline 269 & 爬行纲 & Reptilia & 龟鳖目 & Testudoformes & 龟科 & Emydidae & 大头乌龟 & Chinemys megalocephala & se & 乌龟的同物异名 \\
\hline 270 & 爬行纲 & Reptilia & 龟鳖目 & Testudoformes & 龟科 & Emydidae & 黑颈乌龟 & Chinemys nigricans & sg & \\
\hline 271 & 爬行纲 & Reptilia & 龟鳖目 & Testudoformes & 龟科 & Emydidae & 乌龟 & Chinemys reevesii & sm & \\
\hline 272 & 爬行纲 & Reptilia & 龟鳖目 & Testudoformes & 龟科 & Emydidae & 马来闭壳龟 & Cuora amboinensis & wa & 国内无分布 \\
\hline 273 & 爬行纲 & Reptilia & 龟鳖目 & Testudoformes & 龟科 & Emydidae & 金头闭壳龟 & Cuora aurocapitata & si & \\
\hline 274 & 爬行纲 & Reptilia & 龟鳖目 & Testudoformes & 龟科 & Emydidae & 黄缘闭壳龟 & Cuora flavomarginata & $\mathrm{sc}$ & \\
\hline 275 & 爬行纲 & Reptilia & 龟鳖目 & Testudoformes & 龟科 & Emydidae & 黄额闭壳龟 & Cuora galbinifrons & wa & \\
\hline 276 & 爬行纲 & Reptilia & 龟鳖目 & Testudoformes & 龟科 & Emydidae & 百色闭壳龟 & Cuora mccordi & $\mathrm{sg}$ & \\
\hline 277 & 爬行纲 & Reptilia & 龟鳖目 & Testudoformes & 龟科 & Emydidae & 潘氏闭壳龟 & Cuora pani & sn & \\
\hline 278 & 爬行纲 & Reptilia & 龟鳖目 & Testudoformes & 龟科 & Emydidae & 三线闭壳龟 & Cuora trifasciata & $\mathrm{sb}$ & \\
\hline 279 & 爬行纲 & Reptilia & 龟鳖目 & Testudoformes & 龟科 & Emydidae & 云南闭壳龟 & Cuora yunnanensis & $\mathrm{sc}$ & \\
\hline 280 & 爬行纲 & Reptilia & 龟鳖目 & Testudoformes & 龟科 & Emydidae & 周氏闭壳龟 & Cuora zhoui & $\mathrm{sb}$ & \\
\hline 281 & 爬行纲 & Reptilia & 龟鳖目 & Testudoformes & 龟科 & Emydidae & 齿缘摄龟 & Cyclemys dentata & wa & 分布存疑 \\
\hline 282 & 爬行纲 & Reptilia & 龟鳖目 & Testudoformes & 龟科 & Emydidae & 地龟 & Geoemyda spengleri & $\mathrm{sc}$ & \\
\hline 283 & 爬行纲 & Reptilia & 龟鳖目 & Testudoformes & 龟科 & Emydidae & 广西拟水龟 & Mauremys guangxiensis & si & 黄喉拟水龟的同物异名 \\
\hline 284 & 爬行纲 & Reptilia & 亀监目 & Testudoformes & 龟科 & Emydidae & 艾氏拟水龟 & Mauremys iversoni & si & 杂交龟 \\
\hline
\end{tabular}


郜二虎, 何杰坤, 王志臣, 徐扬, 唐小平, 江海声. 全国陆生野生动物调查单元区划方案. 生物多样性, 2017, 25 (12): 1321-1330.

http://www.biodiversity-science.net/CN/10.17520/biods.2017135

\begin{tabular}{|c|c|c|c|c|c|c|c|c|c|c|}
\hline $\begin{array}{r}\text { 序号 } \\
\text { No. }\end{array}$ & \multicolumn{2}{|c|}{$\begin{array}{c}\text { 纲 } \\
\text { Class }\end{array}$} & \multicolumn{2}{|c|}{$\begin{array}{c}\text { 目 } \\
\text { Order }\end{array}$} & \multicolumn{2}{|r|}{$\begin{array}{c}\text { 科 } \\
\text { Family }\end{array}$} & \multicolumn{2}{|r|}{$\begin{array}{l}\text { 物种名 } \\
\text { Species }\end{array}$} & \multirow{2}{*}{$\begin{array}{l}\begin{array}{c}\text { 分布型 } \\
\text { Chorotype }\end{array} \\
\text { sa }\end{array}$} & \multirow[t]{2}{*}{$\begin{array}{r}\text { 备注 } \\
\text { Remarks }\end{array}$} \\
\hline 285 & 爬行纲 & Reptilia & 龟鳖目 & Testudoformes & 龟科 & Emydidae & 黄喉拟水龟 & Mauremys mutica & & \\
\hline 286 & 爬行纲 & Reptilia & 龟鳖目 & Testudoformes & 龟科 & Emydidae & 费氏花龟 & Ocadia philippeni & $\mathrm{j}$ & 杂交龟 \\
\hline 287 & 爬行纲 & Reptilia & 龟鳖目 & Testudoformes & 龟科 & Emydidae & 花龟 & Ocadia sinensis & $\mathrm{sb}$ & \\
\hline 288 & 爬行纲 & Reptilia & 龟鳖目 & Testudoformes & 龟科 & Emydidae & 锯缘摄龟 & Pyxidea mouhotii & wb & \\
\hline 289 & 爬行纲 & Reptilia & 龟鳖目 & Testudoformes & 龟科 & Emydidae & 眼斑水龟 & Sacalia bealei & $\mathrm{sc}$ & \\
\hline 290 & 爬行纲 & Reptilia & 龟鳖目 & Testudoformes & 龟科 & Emydidae & 拟眼斑水龟 & Sacalia pseudocellata & $\mathrm{j}$ & 杂交龟 \\
\hline 291 & 爬行纲 & Reptilia & 龟鳖目 & Testudoformes & 龟科 & Emydidae & 四眼斑水龟 & Sacalia quadriocellata & $\mathrm{sb}$ & \\
\hline 292 & 爬行纲 & Reptilia & 龟鳖目 & Testudoformes & 陆龟科 & Testudinidae & 凹甲陆龟 & Manouria impressa & wc & \\
\hline 293 & 爬行纲 & Reptilia & 龟鳖目 & Testudoformes & 陆龟科 & Testudinidae & 缅甸陆龟 & Indotestudo elongata & $\mathrm{wb}$ & \\
\hline 294 & 爬行纲 & Reptilia & 龟鳖目 & Testudoformes & 陆龟科 & Testudinidae & 四爪陆龟 & Testudo horsfieldii & dh & \\
\hline 295 & 爬行纲 & Reptilia & 鲻形目 & Crocodiliformes & 鲻科 & Crocodilidae & 軖 & Alligator sinensis & $\mathrm{s}$ & \\
\hline 296 & 爬行纲 & Reptilia & 鳄形目 & Crocodiliformes & 鰙科 & Crocodilidae & 湾鲇 & Crocodilus porosus & wa & 区域灭绝 \\
\hline 297 & 爬行纲 & Reptilia & 有鳞目 & Squamata & 壁虎科 & Gekkonidae & 隐耳漠虎 & Alsophylax pipiens & $\mathrm{dc}$ & \\
\hline 298 & 爬行纲 & Reptilia & 有鳞目 & Squamata & 壁虎科 & Gekkonidae & 新疆漠虎 & Alsophylax przewalskii & $\mathrm{db}$ & \\
\hline 299 & 爬行纲 & Reptilia & 有鳞目 & Squamata & 壁虎科 & Gekkonidae & 长弯脚虎 & Cyrtopodion elongatus & $\mathrm{db}$ & \\
\hline 300 & 爬行纲 & Reptilia & 有鳞目 & Squamata & 壁虎科 & Gekkonidae & 卡西弯脚虎 & Cyrtopodion khasiensis & he & \\
\hline 301 & 爬行纲 & Reptilia & 有鳞目 & Squamata & 壁虎科 & Gekkonidae & 墨脱弯脚虎 & Cyrtopodion medogensis & he & \\
\hline 302 & 爬行纲 & Reptilia & 有鳞目 & Squamata & 壁虎科 & Gekkonidae & 西藏弯脚虎 & Cyrtopodion tibetanus & hd & \\
\hline 303 & 爬行纲 & Reptilia & 有鳞目 & Squamata & 壁虎科 & Gekkonidae & 截趾虎 & Gehyra mutilata & wa & \\
\hline 304 & 爬行纲 & Reptilia & 有鳞目 & Squamata & 壁虎科 & Gekkonidae & 耳疮壁虎 & Gekko auriverrucosus & $\mathrm{b}$ & \\
\hline 305 & 爬行纲 & Reptilia & 有鳞目 & Squamata & 壁虎科 & Gekkonidae & 中国壁虎 & Gekko chinensis & $\mathrm{sb}$ & \\
\hline 306 & 爬行纲 & Reptilia & 有鳞目 & Squamata & 壁虎科 & Gekkonidae & 大壁虎 & Gekko gecko & wb & \\
\hline
\end{tabular}


郜二虎, 何杰坤, 王志臣, 徐扬, 唐小平, 江海声. 全国陆生野生动物调查单元区划方案. 生物多样性, 2017, 25 (12): 1321-1330.

http://www.biodiversity-science.net/CN/10.17520/biods.2017135

\begin{tabular}{|c|c|c|c|c|c|c|c|c|c|c|}
\hline $\begin{array}{r}\text { 序号 } \\
\text { No. }\end{array}$ & \multicolumn{2}{|c|}{$\begin{array}{c}\text { 纲 } \\
\text { Class }\end{array}$} & \multicolumn{2}{|c|}{$\begin{array}{c}\text { 目 } \\
\text { Order }\end{array}$} & \multicolumn{2}{|r|}{$\begin{array}{c}\text { 科 } \\
\text { Family }\end{array}$} & \multicolumn{2}{|r|}{$\begin{array}{l}\text { 物种名 } \\
\text { Species }\end{array}$} & \multirow{2}{*}{$\begin{array}{l}\begin{array}{c}\text { 分布型 } \\
\text { Chorotype }\end{array} \\
\text { si }\end{array}$} & \multirow[t]{2}{*}{$\begin{array}{c}\text { 备注 } \\
\text { Remarks }\end{array}$} \\
\hline 307 & 爬行纲 & Reptilia & 有鳞目 & Squamata & 壁虎科 & Gekkonidae & 铅山壁虎 & Gekko hokouensis & & \\
\hline 308 & 爬行纲 & Reptilia & 有鳞目 & Squamata & 壁虎科 & Gekkonidae & 多疮壁虎 & Gekko japonicus & $\mathrm{sh}$ & \\
\hline 309 & 爬行纲 & Reptilia & 有鳞目 & Squamata & 壁虎科 & Gekkonidae & 兰屿壁虎 & Gekko kikuchii & $\mathrm{j}$ & \\
\hline 310 & 爬行纲 & Reptilia & 有鳞目 & Squamata & 壁虎科 & Gekkonidae & 蹼趾壁虎 & Gekko subpalmatus & $\mathrm{sh}$ & \\
\hline 311 & 爬行纲 & Reptilia & 有鳞目 & Squamata & 壁虎科 & Gekkonidae & 无蹼壁虎 & Gekko swinhonis & ba & \\
\hline 312 & 爬行纲 & Reptilia & 有鳞目 & Squamata & 壁虎科 & Gekkonidae & 太白壁虎 & Gekko taibaiensis & ba & \\
\hline 313 & 爬行纲 & Reptilia & 有鳞目 & Squamata & 壁虎科 & Gekkonidae & 原尾蚚虎 & Hemidactylus bowringii & $\mathrm{wb}$ & \\
\hline 314 & 爬行纲 & Reptilia & 有鳞目 & Squamata & 壁虎科 & Gekkonidae & 疮尾蜥虎 & Hemidactylus frenatus & wb & \\
\hline 315 & 爬行纲 & Reptilia & 有鳞目 & Squamata & 壁虎科 & Gekkonidae & 台湾蚚虎 & Hemidactylus stejnegeri & wa & \\
\hline 316 & 爬行纲 & Reptilia & 有鳞目 & Squamata & 壁虎科 & Gekkonidae & 云南半叶趾虎 & Hemiphyllodactylus yunnanensis & wc & \\
\hline 317 & 爬行纲 & Reptilia & 有鳞目 & Squamata & 壁虎科 & Gekkonidae & 哀鳞趾虎 & Lepidodactylus lugubris & wa & \\
\hline 318 & 爬行纲 & Reptilia & 有鳞目 & Squamata & 壁虎科 & Gekkonidae & 雅美鳞趾虎 & Lepidodactylus yami & $\mathrm{j}$ & \\
\hline 319 & 爬行纲 & Reptilia & 有鳞目 & Squamata & 壁虎科 & Gekkonidae & 蝎虎 & Platyurus platyurus & wa & \\
\hline 320 & 爬行纲 & Reptilia & 有鳞目 & Squamata & 壁虎科 & Gekkonidae & 新疆沙虎 & Teratoscincus przewalskii & $\mathrm{db}$ & \\
\hline 321 & 爬行纲 & Reptilia & 有鳞目 & Squamata & 壁虎科 & Gekkonidae & 伊犁沙虎 & Teratoscincus scincus & $\mathrm{db}$ & \\
\hline 322 & 爬行纲 & Reptilia & 有鳞目 & Squamata & 睑虎科 & Eublepharidae & 睑虎 & Goniurosaurus lichtenfelderi & wa & \\
\hline 323 & 爬行纲 & Reptilia & 有鳞目 & Squamata & 鬣蚚科 & Agamidae & 长棘蜥 & Acanthosaura armata & wa & \\
\hline 324 & 爬行纲 & Reptilia & 有鳞目 & Squamata & 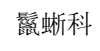 & Agamidae & 丽棘蜥 & Acanthosaura lepidogester & wc & \\
\hline 325 & 爬行纲 & Reptilia & 有鳞目 & Squamata & 甏蜥科 & Agamidae & 短肢树蜥 & Calotes brevipes & hc & \\
\hline 326 & 爬行纲 & Reptilia & 有鳞目 & Squamata & 歖蜥科 & Agamidae & 棕背树蜥 & Calotes emma & wa & \\
\hline 327 & 爬行纲 & Reptilia & 有鳞目 & Squamata & 镾蚚科 & Agamidae & 绿背树蜥 & Calotes jerdoni & he & \\
\hline 328 & 爬行纲 & Reptilia & 有鳞目 & Squamata & 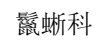 & Agamidae & 蚌西树蜊 & Calotes kakhienensis & $\mathrm{wb}$ & \\
\hline
\end{tabular}


郜二虎, 何杰坤, 王志臣, 徐扬, 唐小平, 江海声. 全国陆生野生动物调查单元区划方案. 生物多样性, 2017, 25 (12): 1321-1330.

http://www.biodiversity-science.net/CN/10.17520/biods.2017135

\begin{tabular}{|c|c|c|c|c|c|c|c|c|c|c|}
\hline \multirow{2}{*}{$\begin{array}{l}\text { 序号 } \\
\text { No. } \\
329\end{array}$} & \multicolumn{2}{|c|}{$\begin{array}{c}\text { 纲 } \\
\text { Class }\end{array}$} & \multicolumn{2}{|c|}{$\begin{array}{c}\text { 目 } \\
\text { Order }\end{array}$} & \multirow{2}{*}{\multicolumn{2}{|c|}{$\begin{array}{c}\text { 科 } \\
\text { Family } \\
\text { Agamidae }\end{array}$}} & \multirow{2}{*}{\multicolumn{2}{|c|}{$\begin{array}{c}\text { 物种名 } \\
\text { Species } \\
\text { Calotes kingdonwardi }\end{array}$}} & \multirow{2}{*}{$\begin{array}{l}\begin{array}{c}\text { 分布型 } \\
\text { Chorotype }\end{array} \\
\text { he }\end{array}$} & \multirow[t]{2}{*}{$\begin{array}{c}\text { 备注 } \\
\text { Remarks }\end{array}$} \\
\hline & 爬行纲 & Reptilia & 有鳞目 & Squamata & & & & & & \\
\hline 330 & 爬行纲 & Reptilia & 有鳞目 & Squamata & 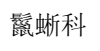 & Agamidae & 墨脱树蚚 & Calotes medogensis & he & \\
\hline 331 & 爬行纲 & Reptilia & 有鳞目 & Squamata & 镾蚚科 & Agamidae & 细鳞树蚚 & Calotes microlepis & wa & \\
\hline 332 & 爬行纲 & Reptilia & 有鳞目 & Squamata & 鬛蜘科 & Agamidae & 白唇树蜥 & Calotes mystaceus & wa & \\
\hline 333 & 爬行纲 & Reptilia & 有鳞目 & Squamata & 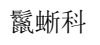 & Agamidae & 变色树蜊 & Calotes versicolor & wb & \\
\hline 334 & 爬行纲 & Reptilia & 有鳞目 & Squamata & 镾蚚科 & Agamidae & 裸耳飞蜊 & Draco blanfordii & wa & \\
\hline 335 & 爬行纲 & Reptilia & 有鳞目 & Squamata & 镾蚚科 & Agamidae & 斑飞虲 & Draco maculatus & wa & \\
\hline 336 & 爬行纲 & Reptilia & 有鳞目 & Squamata & 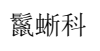 & Agamidae & 长肢龙蜊 & Japalura andersoniana & he & \\
\hline 337 & 爬行纲 & Reptilia & 有鳞目 & Squamata & 㼭蜥科 & Agamidae & 裸耳龙蜊 & Japalura dymondi & hc & \\
\hline 338 & 爬行纲 & Reptilia & 有鳞目 & Squamata & 鬛䖵科 & Agamidae & 草绿龙蜊 & Japalura flaviceps & hc & \\
\hline 339 & 爬行纲 & Reptilia & 有鳞目 & Squamata & 㼭蚚科 & Agamidae & 宜宾龙蜊 & Japalura grahami & si & \\
\hline 340 & 爬行纲 & Reptilia & 有鳞目 & Squamata & 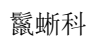 & Agamidae & 喜山龙蜘 & Japalura kumaonensis & ha & \\
\hline 341 & 爬行纲 & Reptilia & 有鳞目 & Squamata & 㼭蚚科 & Agamidae & 溪头龙蜊 & Japalura makii & $\mathrm{j}$ & \\
\hline 342 & 爬行纲 & Reptilia & 有鳞目 & Squamata & 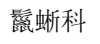 & Agamidae & 米仓山龙蜘 & Japalura micangshanensis & si & \\
\hline 343 & 爬行纲 & Reptilia & 有鳞目 & Squamata & 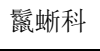 & Agamidae & 琉球龙蜥 & Japalura polygonata & $\mathrm{j}$ & \\
\hline 344 & 爬行纲 & Reptilia & 有鳞目 & Squamata & 㼭蚚科 & Agamidae & 丽纹龙蜥 & Japalura splendida & sh & \\
\hline 345 & 爬行纲 & Reptilia & 有鳞目 & Squamata & 㼭蚚科 & Agamidae & 台湾龙蜘 & Japalura swinhonis & $\mathrm{j}$ & \\
\hline 346 & 爬行纲 & Reptilia & 有鳞目 & Squamata & 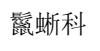 & Agamidae & 四川龙蜊 & Japalura szechwanensis & si & \\
\hline 347 & 爬行纲 & Reptilia & 有鳞目 & Squamata & 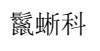 & Agamidae & 昆明龙蜘 & Japalura varcoae & $\mathrm{yb}$ & \\
\hline 348 & 爬行纲 & Reptilia & 有鳞目 & Squamata & 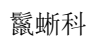 & Agamidae & 云南龙蜘 & Japalura yunnanensis & hc & \\
\hline 349 & 爬行纲 & Reptilia & 有鳞目 & Squamata & 瓺蜥科 & Agamidae & 喜山岩蜊 & Laudakia himalayana & $\mathrm{db}$ & \\
\hline 350 & 爬行纲 & Reptilia & 有鳞目 & Squamata & 鬛蜘科 & Agamidae & 西藏岩蜥 & Laudakia papenfussi & pe & \\
\hline
\end{tabular}


郜二虎, 何杰坤, 王志臣, 徐扬, 唐小平, 江海声. 全国陆生野生动物调查单元区划方案. 生物多样性, 2017, 25 (12): 1321-1330.

http://www.biodiversity-science.net/CN/10.17520/biods.2017135

\begin{tabular}{|c|c|c|c|c|c|c|c|c|c|c|}
\hline $\begin{array}{l}\text { 序号 } \\
\text { No. }\end{array}$ & \multicolumn{2}{|c|}{$\begin{array}{c}\text { 纲 } \\
\text { Class }\end{array}$} & \multicolumn{2}{|c|}{$\begin{array}{c}\text { 目 } \\
\text { Order }\end{array}$} & \multicolumn{2}{|r|}{$\begin{array}{c}\text { 科 } \\
\text { Family }\end{array}$} & \multicolumn{2}{|r|}{$\begin{array}{l}\text { 物种名 } \\
\text { Species }\end{array}$} & \multirow{2}{*}{$\begin{array}{l}\text { 分布型 } \\
\text { Chorotype } \\
\text { hd }\end{array}$} & \multirow[t]{2}{*}{$\begin{array}{c}\text { 备注 } \\
\text { Remarks }\end{array}$} \\
\hline 351 & 爬行纲 & Reptilia & 有鳞目 & Squamata & 鬛䖵科 & Agamidae & 拉萨岩蜊 & Laudakia sacra & & \\
\hline 352 & 爬行纲 & Reptilia & 有鳞目 & Squamata & 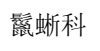 & Agamidae & 新疆岩蜘 & Laudakia stoliczkana & $\mathrm{db}$ & \\
\hline 353 & 爬行纲 & Reptilia & 有鳞目 & Squamata & 䯷蜘科 & Agamidae & 塔里木岩蜥 & Laudakia tarimensis & $\mathrm{db}$ & 新疆岩蜥的同物异名 \\
\hline 354 & 爬行纲 & Reptilia & 有鳞目 & Squamata & 鬛蜘科 & Agamidae & 南亚岩蜘 & Laudakia tuberculata & $\mathrm{ph}$ & \\
\hline 355 & 爬行纲 & Reptilia & 有鳞目 & Squamata & 甏蚚科 & Agamidae & 吴氏岩蜊 & Laudakia wui & he & \\
\hline 356 & 爬行纲 & Reptilia & 有鳞目 & Squamata & 镾蚚科 & Agamidae & 蜡皮蜘 & Leiolepis reevesii & wa & \\
\hline 357 & 爬行纲 & Reptilia & 有鳞目 & Squamata & 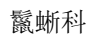 & Agamidae & 异鳞虲 & Oriocalotes paulus & ha & \\
\hline 358 & 爬行纲 & Reptilia & 有鳞目 & Squamata & 髮蜥科 & Agamidae & 白条沙蜊 & Phrynocephalus albolineatus & $\mathrm{dm}$ & 乌拉尔沙蚚黑腹亚种 \\
\hline 359 & 爬行纲 & Reptilia & 有鳞目 & Squamata & 㼭蜥科 & Agamidae & 叶城沙蜥 & Phrynocephalus axillaris & $\mathrm{db}$ & \\
\hline 360 & 爬行纲 & Reptilia & 有鳞目 & Squamata & 鬛䖵科 & Agamidae & 红尾沙蜊 & Phrynocephalus erythrurus & $\mathrm{p}$ & \\
\hline 361 & 爬行纲 & Reptilia & 有鳞目 & Squamata & 鬛蜊科 & Agamidae & 南疆沙蜊 & Phrynocephalus forsythii & $\mathrm{db}$ & \\
\hline 362 & 爬行纲 & Reptilia & 有鳞目 & Squamata & 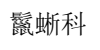 & Agamidae & 草原沙蜘 & Phrynocephalus frontalis & $\mathrm{dg}$ & 荒漠沙蜥的同物异名 \\
\hline 363 & 爬行纲 & Reptilia & 有鳞目 & Squamata & 㼭蚚科 & Agamidae & 奇台沙蜊 & Phrynocephalus grumgrzimailoi & $\mathrm{dc}$ & \\
\hline 364 & 爬行纲 & Reptilia & 有鳞目 & Squamata & 鬛䖵科 & Agamidae & 乌拉尔沙蜘 & Phrynocephalus guttatus & $\mathrm{dc}$ & \\
\hline 365 & 爬行纲 & Reptilia & 有鳞目 & Squamata & 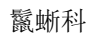 & Agamidae & 旱地沙蜊 & Phrynocephalus helioscopus & dc & \\
\hline 366 & 爬行纲 & Reptilia & 有鳞目 & Squamata & 鼠蜥科 & Agamidae & 红原沙蜥 & Phrynocephalus hongyuanensis & hc & 青海沙蜥的同物异名 \\
\hline 367 & 爬行纲 & Reptilia & 有鳞目 & Squamata & 甏蚚科 & Agamidae & 无斑沙蜊 & Phrynocephalus immaculatus & d & 变色沙蜥的同物异名 \\
\hline 368 & 爬行纲 & Reptilia & 有鳞目 & Squamata & 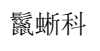 & Agamidae & 白梢沙蜊 & Phrynocephalus koslowi & df & 叶城沙蜥的同物异名 \\
\hline 369 & 爬行纲 & Reptilia & 有鳞目 & Squamata & 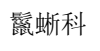 & Agamidae & 大耳沙虲 & Phrynocephalus mystaceus & $\mathrm{dh}$ & \\
\hline 370 & 爬行纲 & Reptilia & 有鳞目 & Squamata & 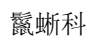 & Agamidae & 宽鼻沙虲 & Phrynocephalus nasatus & $\mathrm{da}$ & \\
\hline 371 & 爬行纲 & Reptilia & 有鳞目 & Squamata & 䯿蜥科 & Agamidae & 荒漠沙蜥 & Phrynocephalus przewalskii & $\mathrm{db}$ & \\
\hline 372 & 爬行纲 & Reptilia & 有鳞目 & Squamata & 鬛蜊科 & Agamidae & 西藏沙蜥 & Phrynocephalus theobaldi & hd & \\
\hline
\end{tabular}


郜二虎, 何杰坤, 王志臣, 徐扬, 唐小平, 江海声. 全国陆生野生动物调查单元区划方案. 生物多样性, 2017, 25 (12): 1321-1330.

http://www.biodiversity-science.net/CN/10.17520/biods.2017135

\begin{tabular}{|c|c|c|c|c|c|c|c|c|c|c|}
\hline $\begin{array}{l}\text { 序号 } \\
\text { No. }\end{array}$ & \multicolumn{2}{|c|}{$\begin{array}{c}\text { 纲 } \\
\text { Class }\end{array}$} & \multicolumn{2}{|c|}{$\begin{array}{c}\text { 目 } \\
\text { Order }\end{array}$} & \multicolumn{2}{|r|}{$\begin{array}{c}\text { 科 } \\
\text { Family }\end{array}$} & \multicolumn{2}{|r|}{$\begin{array}{l}\text { 物种名 } \\
\text { Species }\end{array}$} & \multirow{2}{*}{$\begin{array}{l}\begin{array}{c}\text { 分布型 } \\
\text { Chorotype }\end{array} \\
\mathrm{di}\end{array}$} & \multirow[t]{2}{*}{$\begin{array}{c}\text { 备注 } \\
\text { Remarks }\end{array}$} \\
\hline 373 & 爬行纲 & Reptilia & 有鳞目 & Squamata & 鬛䖵科 & Agamidae & 变色沙蜊 & Phrynocephalus versicolor & & \\
\hline 374 & 爬行纲 & Reptilia & 有鳞目 & Squamata & 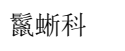 & Agamidae & 青海沙蜘 & Phrynocephalus vlangalii & $\mathrm{pc}$ & \\
\hline 375 & 爬行纲 & Reptilia & 有鳞目 & Squamata & 㼭蜘科 & Agamidae & 泽当沙蜥 & Phrynocephalus zetangensis & hd & 西藏沙蚚的同物异名 \\
\hline 376 & 爬行纲 & Reptilia & 有鳞目 & Squamata & 鬛蜘科 & Agamidae & 长髵蜥 & Physignathus cocincinus & wb & \\
\hline 377 & 爬行纲 & Reptilia & 有鳞目 & Squamata & 甏蚚科 & Agamidae & 喉褶蜥 & Ptyctolaemus gularis & he & \\
\hline 378 & 爬行纲 & Reptilia & 有鳞目 & Squamata & 镾蚚科 & Agamidae & 草原蜥 & Trapelus sanguinolenta & $\mathrm{dh}$ & \\
\hline 379 & 爬行纲 & Reptilia & 有鳞目 & Squamata & 蛇蚚科 & Anguidae & 台湾脆蛇 & Ophisaurus formosensis & $\mathrm{j}$ & 脆蛇蚚的同物异名 \\
\hline 380 & 爬行纲 & Reptilia & 有鳞目 & Squamata & 蛇蚚科 & Anguidae & 细脆蛇 & Ophisaurus gracilis & wb & \\
\hline 381 & 爬行纲 & Reptilia & 有鳞目 & Squamata & 蛇蚚科 & Anguidae & 海南脆蛇 & Ophisaurus hainanensis & $\mathrm{j}$ & \\
\hline 382 & 爬行纲 & Reptilia & 有鳞目 & Squamata & 蛇蚚科 & Anguidae & 脆蛇 & Ophisaurus harti & $\mathrm{sb}$ & \\
\hline 383 & 爬行纲 & Reptilia & 有鳞目 & Squamata & 魾蚚科 & Shinisauridae & 䲞虲 & Shinisaurus crocodilurus & si & \\
\hline 384 & 爬行纲 & Reptilia & 有鳞目 & Squamata & 巨蜊科 & Varanidae & 孟加拉巨蚚 & Varanus bengalensis & sa & \\
\hline 385 & 爬行纲 & Reptilia & 有鳞目 & Squamata & 巨蚚科 & Varanidae & 圆鼻巨蜊 & Varanus salvator & wa & \\
\hline 386 & 爬行纲 & Reptilia & 有鳞目 & Squamata & 双足蜊科 & Dibamidae & 香港双足蜊 & Dibamus bogadeki & $\mathrm{j}$ & \\
\hline 387 & 爬行纲 & Reptilia & 有鳞目 & Squamata & 双足蜊科 & Dibamidae & 白尾双足虲 & Dibamus bourreti & sg & \\
\hline 388 & 爬行纲 & Reptilia & 有鳞目 & Squamata & 蚚蜴科 & Lacertidae & 丽斑麻蜥 & Eremias argus & xe & \\
\hline 389 & 爬行纲 & Reptilia & 有鳞目 & Squamata & 蚚蜴科 & Lacertidae & 敏麻虲 & Eremias arguta & da & \\
\hline 390 & 爬行纲 & Reptilia & 有鳞目 & Squamata & 蜊蜴科 & Lacertidae & 山地麻蜊 & Eremias brenchleyi & $\mathrm{x}$ & \\
\hline 391 & 爬行纲 & Reptilia & 有鳞目 & Squamata & 蜊蜴科 & Lacertidae & 网纹麻蜊 & Eremias grammica & df & \\
\hline 392 & 爬行纲 & Reptilia & 有鳞目 & Squamata & 蜊蜴科 & Lacertidae & 密点麻蜊 & Eremias multiocellata & $\mathrm{dg}$ & \\
\hline 393 & 爬行纲 & Reptilia & 有鳞目 & Squamata & 蚚蜴科 & Lacertidae & 荒漠麻蜊 & Eremias przewalskii & $\mathrm{db}$ & \\
\hline 394 & 爬行纲 & Reptilia & 有鳞目 & Squamata & 蝴蜴科 & Lacertidae & 快步麻蜥 & Eremias velox & $\mathrm{dc}$ & \\
\hline
\end{tabular}


郜二虎, 何杰坤, 王志臣, 徐扬, 唐小平, 江海声. 全国陆生野生动物调查单元区划方案. 生物多样性, 2017, 25 (12): 1321-1330.

http://www.biodiversity-science.net/CN/10.17520/biods.2017135

\begin{tabular}{|c|c|c|c|c|c|c|c|c|c|c|}
\hline \multirow{2}{*}{$\begin{array}{l}\text { 序号 } \\
\text { No. } \\
395\end{array}$} & \multicolumn{2}{|c|}{$\begin{array}{c}\text { 纲 } \\
\text { Class }\end{array}$} & \multicolumn{2}{|c|}{$\begin{array}{c}\text { 目 } \\
\text { Order }\end{array}$} & \multicolumn{2}{|r|}{$\begin{array}{c}\text { 科 } \\
\text { Family }\end{array}$} & \multicolumn{2}{|r|}{$\begin{array}{l}\text { 物种名 } \\
\text { Species }\end{array}$} & \multirow{2}{*}{$\begin{array}{l}\begin{array}{c}\text { 分布型 } \\
\text { Chorotype }\end{array} \\
\mathrm{db}\end{array}$} & \multirow[t]{2}{*}{$\begin{array}{c}\text { 备注 } \\
\text { Remarks }\end{array}$} \\
\hline & 爬行纲 & Reptilia & 有鳞目 & Squamata & 蜊蜴科 & Lacertidae & 虫纹麻蜊 & Eremias vermiculata & & \\
\hline 396 & 爬行纲 & Reptilia & 有鳞目 & Squamata & 蚚蜴科 & Lacertidae & 捷蜘蜴 & Lacerta agilis & $\mathrm{ub}$ & \\
\hline 397 & 爬行纲 & Reptilia & 有鳞目 & Squamata & 蜊蜴科 & Lacertidae & 胎生蚚蜴 & Lacerta vivipara & uc & \\
\hline 398 & 爬行纲 & Reptilia & 有鳞目 & Squamata & 蚚蜴科 & Lacertidae & 峨眉地蜊 & Platyplacopus intermedius & he & \\
\hline 399 & 爬行纲 & Reptilia & 有鳞目 & Squamata & 蚚蜴科 & Lacertidae & 台湾地蜊 & Platyplacopus kuehnei & si & \\
\hline 400 & 爬行纲 & Reptilia & 有鳞目 & Squamata & 蚚蜴科 & Lacertidae & 崇安地蜊 & Platyplacopus sylvaticus & si & \\
\hline 401 & 爬行纲 & Reptilia & 有鳞目 & Squamata & 蚚蜴科 & Lacertidae & 黑龙江草蜘 & Takydromus amurensis & $\mathrm{kb}$ & \\
\hline 402 & 爬行纲 & Reptilia & 有鳞目 & Squamata & 蚚蜴科 & Lacertidae & 雪山草蜊 & Takydromus hsuehshanensis & $\mathrm{j}$ & \\
\hline 403 & 爬行纲 & Reptilia & 有鳞目 & Squamata & 蚚蜴科 & Lacertidae & 北草蜊 & Takydromus septentrionalis & e & \\
\hline 404 & 爬行纲 & Reptilia & 有鳞目 & Squamata & 蚚蜴科 & Lacertidae & 南草蜊 & Takydromus sexlineatus & wc & \\
\hline 405 & 爬行纲 & Reptilia & 有鳞目 & Squamata & 蚚蜴科 & Lacertidae & 白条草蜘 & Takydromus wolteri & eb & \\
\hline 406 & 爬行纲 & Reptilia & 有鳞目 & Squamata & 石龙子科 & Scincidae & 阿赖山裂脸蚚 & Asymblepharus alaicus & $\mathrm{db}$ & \\
\hline 407 & 爬行纲 & Reptilia & 有鳞目 & Squamata & 石龙子科 & Scincidae & 光蜥 & Ateuchosaurus chinensis & wb & \\
\hline 408 & 爬行纲 & Reptilia & 有鳞目 & Squamata & 石龙子科 & Scincidae & 岩岸岛蜊 & Emoia atrocostata & $\mathrm{j}$ & \\
\hline 409 & 爬行纲 & Reptilia & 有鳞目 & Squamata & 石龙子科 & Scincidae & 黄纹石龙子 & Eumeces capito & ba & \\
\hline 410 & 爬行纲 & Reptilia & 有鳞目 & Squamata & 石龙子科 & Scincidae & 中国石龙子 & Eumeces chinensis & sm & \\
\hline 411 & 爬行纲 & Reptilia & 有鳞目 & Squamata & 石龙子科 & Scincidae & 蓝尾石龙子 & Eumeces elegans & sf & \\
\hline 412 & 爬行纲 & Reptilia & 有鳞目 & Squamata & 石龙子科 & Scincidae & 刘氏石龙子 & Eumeces liui & si & \\
\hline 413 & 爬行纲 & Reptilia & 有鳞目 & Squamata & 石龙子科 & Scincidae & 崇安石龙子 & Eumeces popei & si & \\
\hline 414 & 爬行纲 & Reptilia & 有鳞目 & Squamata & 石龙子科 & Scincidae & 四线石龙子 & Eumeces quadrilineatus & $\mathrm{wb}$ & \\
\hline 415 & 爬行纲 & Reptilia & 有鳞目 & Squamata & 石龙子科 & Scincidae & 大渡石龙子 & Eumeces tunganus & hc & \\
\hline 416 & 爬行纲 & Reptilia & 有鳞目 & Squamata & 石龙子科 & Scincidae & 长尾南蜥 & Mabuya longicaudata & wa & \\
\hline
\end{tabular}


郜二虎, 何杰坤, 王志臣, 徐扬, 唐小平, 江海声. 全国陆生野生动物调查单元区划方案. 生物多样性, 2017, 25 (12): 1321-1330.

http://www.biodiversity-science.net/CN/10.17520/biods.2017135

\begin{tabular}{|c|c|c|c|c|c|c|c|c|c|c|}
\hline \multirow{2}{*}{$\begin{array}{l}\text { 序号 } \\
\text { No. } \\
417\end{array}$} & \multicolumn{2}{|c|}{$\begin{array}{c}\text { 纲 } \\
\text { Class }\end{array}$} & \multicolumn{2}{|c|}{$\begin{array}{c}\text { 目 } \\
\text { Order }\end{array}$} & \multicolumn{2}{|r|}{$\begin{array}{c}\text { 科 } \\
\text { Family }\end{array}$} & \multicolumn{2}{|r|}{$\begin{array}{l}\text { 物种名 } \\
\text { Species }\end{array}$} & \multirow{2}{*}{$\begin{array}{l}\begin{array}{c}\text { 分布型 } \\
\text { Chorotype }\end{array} \\
\text { wa }\end{array}$} & \multirow[t]{2}{*}{$\begin{array}{c}\text { 备注 } \\
\text { Remarks }\end{array}$} \\
\hline & 爬行纲 & Reptilia & 有鳞目 & Squamata & 石龙子科 & Scincidae & 多棱南蜊 & Mabuya multicarinata & & \\
\hline 418 & 爬行纲 & Reptilia & 有鳞目 & Squamata & 石龙子科 & Scincidae & 多线南蜊 & Mabuya multifasciata & wa & \\
\hline 419 & 爬行纲 & Reptilia & 有鳞目 & Squamata & 石龙子科 & Scincidae & 昆明滑蜥 & Scincella barbouri & hc & \\
\hline 420 & 爬行纲 & Reptilia & 有鳞目 & Squamata & 石龙子科 & Scincidae & 长肢滑蜊 & Scincella doriae & hc & \\
\hline 421 & 爬行纲 & Reptilia & 有鳞目 & Squamata & 石龙子科 & Scincidae & 台湾滑蜊 & Scincella formosensis & $\mathrm{j}$ & \\
\hline 422 & 爬行纲 & Reptilia & 有鳞目 & Squamata & 石龙子科 & Scincidae & 喜山滑蜊 & Scincella himalayana & ha & \\
\hline 423 & 爬行纲 & Reptilia & 有鳞目 & Squamata & 石龙子科 & Scincidae & 桓仁滑蜘 & Scincella huanrenensis & $\mathrm{kb}$ & \\
\hline 424 & 爬行纲 & Reptilia & 有鳞目 & Squamata & 石龙子科 & Scincidae & 拉达克滑虲 & Scincella ladacensis & ha & \\
\hline 425 & 爬行纲 & Reptilia & 有鳞目 & Squamata & 石龙子科 & Scincidae & 宁波滑蜥 & Scincella modesta & sh & \\
\hline 426 & 爬行纲 & Reptilia & 有鳞目 & Squamata & 石龙子科 & Scincidae & 山滑蜊 & Scincella monticola & hc & \\
\hline 427 & 爬行纲 & Reptilia & 有鳞目 & Squamata & 石龙子科 & Scincidae & 康定滑蜊 & Scincella potanini & hc & \\
\hline 428 & 爬行纲 & Reptilia & 有鳞目 & Squamata & 石龙子科 & Scincidae & 西域滑蜘 & Scincella przewalskii & d & \\
\hline 429 & 爬行纲 & Reptilia & 有鳞目 & Squamata & 石龙子科 & Scincidae & 南滑虲 & Scincella reevesii & we & \\
\hline 430 & 爬行纲 & Reptilia & 有鳞目 & Squamata & 石龙子科 & Scincidae & 瓦山滑蜊 & Scincella schmidti & hc & \\
\hline 431 & 爬行纲 & Reptilia & 有鳞目 & Squamata & 石龙子科 & Scincidae & 锡金滑蜥 & Scincella sikimmensis & ha & \\
\hline 432 & 爬行纲 & Reptilia & 有鳞目 & Squamata & 石龙子科 & Scincidae & 秦岭滑蜥 ～～～～～～ & Scincella tsinlingensis & d & \\
\hline 433 & 爬行纲 & Reptilia & 有鳞目 & Squamata & 石龙子科 & Scincidae & 墨脱蜓蜥 & Sphenomorphus courcyanus & ha & \\
\hline 434 & 爬行纲 & Reptilia & 有鳞目 & Squamata & 石龙子科 & Scincidae & 股鳞蜓蚚 & Sphenomorphus incognitus & sc & \\
\hline 435 & 爬行纲 & Reptilia & 有鳞目 & Squamata & 石龙子科 & Scincidae & 铜蜓虲 & Sphenomorphus indicus & we & \\
\hline 436 & 爬行纲 & Reptilia & 有鳞目 & Squamata & 石龙子科 & Scincidae & 斑蜓虲 & Sphenomorphus maculatus & we & \\
\hline 437 & 爬行纲 & Reptilia & 有鳞目 & Squamata & 石龙子科 & Scincidae & 台湾蜓蜊 & Sphenomorphus taiwanensis & $\mathrm{j}$ & \\
\hline 438 & 爬行纲 & Reptilia & 有鳞目 & Squamata & 石龙子科 & Scincidae & 缅甸棱蚚 & Tropidophorus berdmorei & wa & \\
\hline
\end{tabular}


郜二虎, 何杰坤, 王志臣, 徐扬, 唐小平, 江海声. 全国陆生野生动物调查单元区划方案. 生物多样性, 2017, 25 (12): 1321-1330.

http://www.biodiversity-science.net/CN/10.17520/biods.2017135

\begin{tabular}{|c|c|c|c|c|c|c|c|c|c|c|}
\hline \multirow{2}{*}{$\begin{array}{l}\text { 序号 } \\
\text { No. } \\
439\end{array}$} & \multicolumn{2}{|c|}{$\begin{array}{c}\text { 纲 } \\
\text { Class }\end{array}$} & \multicolumn{2}{|c|}{$\begin{array}{c}\text { 目 } \\
\text { Order }\end{array}$} & \multicolumn{2}{|r|}{$\begin{array}{c}\text { 科 } \\
\text { Family }\end{array}$} & \multicolumn{2}{|r|}{$\begin{array}{l}\text { 物种名 } \\
\text { Species }\end{array}$} & \multirow{2}{*}{$\begin{array}{l}\begin{array}{c}\text { 分布型 } \\
\text { Chorotype }\end{array} \\
\mathrm{sg}\end{array}$} & \multirow[t]{2}{*}{$\begin{array}{c}\text { 备注 } \\
\text { Remarks }\end{array}$} \\
\hline & 爬行纲 & Reptilia & 有鳞目 & Squamata & 石龙子科 & Scincidae & 广西棱蜊 & Tropidophorus guangxiensis & & \\
\hline 440 & 爬行纲 & Reptilia & 有鳞目 & Squamata & 石龙子科 & Scincidae & 海南棱蜊 & Tropidophorus hainanus & $\mathrm{sb}$ & \\
\hline 441 & 爬行纲 & Reptilia & 有鳞目 & Squamata & 石龙子科 & Scincidae & 中国棱蜊 & Tropidophorus sinicus & $\mathrm{sb}$ & \\
\hline 442 & 爬行纲 & Reptilia & 有鳞目 & Squamata & 盲蛇科 & Typhlopidae & 白头钩盲蛇 & Ramphotyphlops albiceps & wa & \\
\hline 443 & 爬行纲 & Reptilia & 有鳞目 & Squamata & 盲蛇科 & Typhlopidae & 钩盲蛇 & Ramphotyphlops braminus & wc & \\
\hline 444 & 爬行纲 & Reptilia & 有鳞目 & Squamata & 盲蛇科 & Typhlopidae & 大盲蛇 & Typhlops diardii & wb & \\
\hline 445 & 爬行纲 & Reptilia & 有鳞目 & Squamata & 盲蛇科 & Typhlopidae & 恒春盲蛇 & Typhlops koshunensis & $\mathrm{j}$ & \\
\hline 446 & 爬行纲 & Reptilia & 有鳞目 & Squamata & 闪鳞蛇科 & Xenopeltidae & 海南闪鳞蛇 & Xenopeltis hainanensis & $\mathrm{se}$ & \\
\hline 447 & 爬行纲 & Reptilia & 有鳞目 & Squamata & 闪鳞蛇科 & Xenopeltidae & 闪鳞蛇 & Xenopeltis unicolor & wa & \\
\hline 448 & 爬行纲 & Reptilia & 有鳞目 & Squamata & 盾尾蛇科 & Uropeltidae & 红尾筒蛇 & Cylindrophis ruffus & wa & \\
\hline 449 & 爬行纲 & Reptilia & 有鳞目 & Squamata & 蟒科 & Boidae & 东方沙蟒 & Eryx tataricus & $\mathrm{dc}$ & \\
\hline 450 & 爬行纲 & Reptilia & 有鳞目 & Squamata & 蟒科 & Boidae & 蟒蛇 & Python molurus & wc & \\
\hline 451 & 爬行纲 & Reptilia & 有鳞目 & Squamata & 游蛇科 & Colubridae & 青脊蛇 & Achalinus ater & si & \\
\hline 452 & 爬行纲 & Reptilia & 有鳞目 & Squamata & 游蛇科 & Colubridae & 海南脊蛇 & Achalinus hainanus & $\mathrm{j}$ & \\
\hline 453 & 爬行纲 & Reptilia & 有鳞目 & Squamata & 游蛇科 & Colubridae & 井冈山脊蛇 & Achalinus jinggangensis & si & \\
\hline 454 & 爬行纲 & Reptilia & 有鳞目 & Squamata & 游蛇科 & Colubridae & 美姑脊蛇 & Achalinus meiguensis & hc & \\
\hline 455 & 爬行纲 & Reptilia & 有鳞目 & Squamata & 游蛇科 & Colubridae & 阿里山脊蛇 & Achalinus niger & $\mathrm{j}$ & \\
\hline 456 & 爬行纲 & Reptilia & 有鳞目 & Squamata & 游蛇科 & Colubridae & 棕脊蛇 & Achalinus rufescens & sd & \\
\hline 457 & 爬行纲 & Reptilia & 有鳞目 & Squamata & 游蛇科 & Colubridae & 黑脊蛇 & Achalinus spinalis & sd & \\
\hline 458 & 爬行纲 & Reptilia & 有鳞目 & Squamata & 游蛇科 & Colubridae & 绿瘦蛇 & Ahaetulla prasina & wc & \\
\hline 459 & 爬行纲 & Reptilia & 有鳞目 & Squamata & 游蛇科 & Colubridae & 无题鳞腹链蛇 & Amphiesma atemporalis & sc & \\
\hline 460 & 爬行纲 & Reptilia & 有鳞目 & Squamata & 游蛇科 & Colubridae & 黑带腹链蛇 & Amphiesma bitaeniata & sa & \\
\hline
\end{tabular}


郜二虎, 何杰坤, 王志臣, 徐扬, 唐小平, 江海声. 全国陆生野生动物调查单元区划方案. 生物多样性, 2017, 25 (12): 1321-1330.

http://www.biodiversity-science.net/CN/10.17520/biods.2017135

\begin{tabular}{|c|c|c|c|c|c|c|c|c|c|c|}
\hline \multirow{2}{*}{$\begin{array}{l}\text { 序号 } \\
\text { No. } \\
461\end{array}$} & \multicolumn{2}{|c|}{$\begin{array}{c}\text { 纲 } \\
\text { Class }\end{array}$} & \multicolumn{2}{|c|}{$\begin{array}{c}\text { 目 } \\
\text { Order }\end{array}$} & \multirow{2}{*}{\multicolumn{2}{|c|}{$\begin{array}{c}\text { 科 } \\
\text { Family } \\
\text { Colubridae }\end{array}$}} & \multirow{2}{*}{\multicolumn{2}{|c|}{$\begin{array}{c}\text { 物种名 } \\
\text { Species } \\
\text { Amphiesma boulengeri }\end{array}$}} & \multirow{2}{*}{$\begin{array}{l}\text { 分布型 } \\
\text { Chorotype } \\
\mathrm{sb}\end{array}$} & \multirow[t]{2}{*}{$\begin{array}{c}\text { 备注 } \\
\text { Remarks }\end{array}$} \\
\hline & 爬行纲 & Reptilia & 有鳞目 & Squamata & & & & & & \\
\hline 462 & 爬行纲 & Reptilia & 有鳞目 & Squamata & 游蛇科 & Colubridae & 锈链腹链蛇 & Amphiesma craspedogaster & sh & \\
\hline 463 & 爬行纲 & Reptilia & 有鳞目 & Squamata & 游蛇科 & Colubridae & 棕网腹链蛇 & Amphiesma johannis & hc & \\
\hline 464 & 爬行纲 & Reptilia & 有鳞目 & Squamata & 游蛇科 & Colubridae & 卡西腹链蛇 & Amphiesma khasiensis & he & \\
\hline 465 & 爬行纲 & Reptilia & 有鳞目 & Squamata & 游蛇科 & Colubridae & 台北腹链蛇 & Amphiesma miyajimae & $\mathrm{j}$ & \\
\hline 466 & 爬行纲 & Reptilia & 有鳞目 & Squamata & 游蛇科 & Colubridae & 腹斑腹链蛇 & Amphiesma modesta & wb & \\
\hline 467 & 爬行纲 & Reptilia & 有鳞目 & Squamata & 游蛇科 & Colubridae & 八线腹链蛇 & Amphiesma octolineata & hc & \\
\hline 468 & 爬行纲 & Reptilia & 有鳞目 & Squamata & 游蛇科 & Colubridae & 丽纹腹链蛇 & Amphiesma optata & y & \\
\hline 469 & 爬行纲 & Reptilia & 有鳞目 & Squamata & 游蛇科 & Colubridae & 双带腹链蛇 & Amphiesma parallela & ha & \\
\hline 470 & 爬行纲 & Reptilia & 有鳞目 & Squamata & 游蛇科 & Colubridae & 平头腹链蛇 & Amphiesma platyceps & ha & \\
\hline 471 & 爬行纲 & Reptilia & 有鳞目 & Squamata & 游蛇科 & Colubridae & 坡普腹链蛇 & Amphiesma popei & $\mathrm{sc}$ & \\
\hline 472 & 爬行纲 & Reptilia & 有鳞目 & Squamata & 游蛇科 & Colubridae & 棕黑腹链蛇 & Amphiesma sauteri & sd & \\
\hline 473 & 爬行纲 & Reptilia & 有鳞目 & Squamata & 游蛇科 & Colubridae & 草腹链蛇 & Amphiesma stotata & we & \\
\hline 474 & 爬行纲 & Reptilia & 有鳞目 & Squamata & 游蛇科 & Colubridae & 缅北腹链蛇 & Amphiesma venningi & $\mathrm{sa}$ & \\
\hline 475 & 爬行纲 & Reptilia & 有鳞目 & Squamata & 游蛇科 & Colubridae & 东亚腹链蛇 & Amphiesma vibakari & $\mathrm{k}$ & \\
\hline 476 & 爬行纲 & Reptilia & 有鳞目 & Squamata & 游蛇科 & Colubridae & 白框蛇 & Amphiesmoides ornaticeps & $\mathrm{sb}$ & \\
\hline 477 & 爬行纲 & Reptilia & 有鳞目 & Squamata & 游蛇科 & Colubridae & 滇西蛇 & Atretium yunnanensis & $\mathrm{sa}$ & \\
\hline 478 & 爬行纲 & Reptilia & 有鳞目 & Squamata & 游蛇科 & Colubridae & 珠光蛇 & Blythia reticulata & he & \\
\hline 479 & 爬行纲 & Reptilia & 有鳞目 & Squamata & 游蛇科 & Colubridae & 绿林蛇 & Boiga cyanea & wa & \\
\hline 480 & 爬行纲 & Reptilia & 有鳞目 & Squamata & 游蛇科 & Colubridae & 广西林蛇 & Boiga guangxiensis & wa & \\
\hline 481 & 爬行纲 & Reptilia & 有鳞目 & Squamata & 游蛇科 & Colubridae & 绞花林蛇 & Boiga kraepelini & sc & \\
\hline 482 & 爬行纲 & Reptilia & 有鳞目 & Squamata & 游蛇科 & Colubridae & 繁花林蛇 & Boiga multomaculata & wc & \\
\hline
\end{tabular}


郜二虎, 何杰坤, 王志臣, 徐扬, 唐小平, 江海声. 全国陆生野生动物调查单元区划方案. 生物多样性, 2017, 25 (12): 1321-1330,

http://www.biodiversity-science.net/CN/10.17520/biods.2017135

\begin{tabular}{|c|c|c|c|c|c|c|c|c|c|c|}
\hline \multirow{2}{*}{$\begin{array}{l}\text { 序号 } \\
\text { No. } \\
483\end{array}$} & \multicolumn{2}{|c|}{$\begin{array}{c}\text { 纲 } \\
\text { Class }\end{array}$} & \multicolumn{2}{|c|}{$\begin{array}{c}\text { 目 } \\
\text { Order }\end{array}$} & \multirow{2}{*}{\multicolumn{2}{|c|}{$\begin{array}{l}\text { 科 } \\
\text { Family } \\
\text { Colubridae }\end{array}$}} & \multirow{2}{*}{\multicolumn{2}{|c|}{$\begin{array}{l}\text { 物种名 } \\
\text { Species } \\
\text { Calamaria pavimentata }\end{array}$}} & \multirow{2}{*}{$\begin{array}{l}\text { 分布型 } \\
\text { Chorotype } \\
\text { wd }\end{array}$} & \multirow[t]{2}{*}{$\begin{array}{c}\text { 备注 } \\
\text { Remarks }\end{array}$} \\
\hline & 爬行纲 & Reptilia & 有鳞目 & Squamata & & & & & & \\
\hline 484 & 爬行纲 & Reptilia & 有鳞目 & Squamata & 游蛇科 & Colubridae & 针尾两头蛇 & Calamaria septentrionalis & sc & \\
\hline 485 & 爬行纲 & Reptilia & 有鳞目 & Squamata & 游蛇科 & Colubridae & 云南两头蛇 & Calamaria yunnanensis & hc & \\
\hline 486 & 爬行纲 & Reptilia & 有鳞目 & Squamata & 游蛇科 & Colubridae & 金花蛇 & Chrysopelea ornata & wa & \\
\hline 487 & 爬行纲 & Reptilia & 有鳞目 & Squamata & 游蛇科 & Colubridae & 花脊游蛇 & Coluber ravergieri & dh & \\
\hline 488 & 爬行纲 & Reptilia & 有鳞目 & Squamata & 游蛇科 & Colubridae & 黄脊游蛇 & Coluber spinalis & ub & \\
\hline 489 & 爬行纲 & Reptilia & 有鳞目 & Squamata & 游蛇科 & Colubridae & 纯绿翠青蛇 & Cyclophiops doriae & sa & \\
\hline 490 & 爬行纲 & Reptilia & 有鳞目 & Squamata & 游蛇科 & Colubridae & 翠青蛇 & Cyclophiops major & sv & \\
\hline 491 & 爬行纲 & Reptilia & 有鳞目 & Squamata & 游蛇科 & Colubridae & 横纹翠青蛇 & Cyclophiops multicinctus & $\mathrm{sc}$ & \\
\hline 492 & 爬行纲 & Reptilia & 有鳞目 & Squamata & 游蛇科 & Colubridae & 喜山过树蛇 & Dendrelaphis gorei & he & \\
\hline 493 & 爬行纲 & Reptilia & 有鳞目 & Squamata & 游蛇科 & Colubridae & 过树蛇 & Dendrelaphis pictus & wa & \\
\hline 494 & 爬行纲 & Reptilia & 有鳞目 & Squamata & 游蛇科 & Colubridae & 八莫过树蛇 & Dendrelaphis subocularis & wa & \\
\hline 495 & 爬行纲 & Reptilia & 有鳞目 & Squamata & 游蛇科 & Colubridae & 黄链蛇 & Dinodon flavozonatum & sc & \\
\hline 496 & 爬行纲 & Reptilia & 有鳞目 & Squamata & 游蛇科 & Colubridae & 粉链蛇 & Dinodon rosozonatum & $\mathrm{j}$ & \\
\hline 497 & 爬行纲 & Reptilia & 有鳞目 & Squamata & 游蛇科 & Colubridae & 赤链蛇 & Dinodon rufozonatum & ed & \\
\hline 498 & 爬行纲 & Reptilia & 有鳞目 & Squamata & 游蛇科 & Colubridae & 白链蛇 & Dinodon septentrionalis & he & \\
\hline 499 & 爬行纲 & Reptilia & 有鳞目 & Squamata & 游蛇科 & Colubridae & 赤峰锦蛇 & Elaphe anomala & ba & \\
\hline 500 & 爬行纲 & Reptilia & 有鳞目 & Squamata & 游蛇科 & Colubridae & 双斑锦蛇 & Elaphe bimaculata & sh & \\
\hline 501 & 爬行纲 & Reptilia & 有鳞目 & Squamata & 游蛇科 & Colubridae & 王锦蛇 & Elaphe carinata & sd & \\
\hline 502 & 爬行纲 & Reptilia & 有鳞目 & Squamata & 游蛇科 & Colubridae & 团花锦蛇 & Elaphe davidi & $\mathrm{m}$ & \\
\hline 503 & 爬行纲 & Reptilia & 有鳞目 & Squamata & 游蛇科 & Colubridae & 白条锦蛇 & Elaphe dione & ub & \\
\hline 504 & 爬行纲 & Reptilia & 有鳞目 & Squamata & 游蛇科 & Colubridae & 灰腹绿锦蛇 & Elaphe frenata & se & \\
\hline
\end{tabular}


郜二虎, 何杰坤, 王志臣, 徐扬, 唐小平, 江海声. 全国陆生野生动物调查单元区划方案. 生物多样性, 2017, 25 (12): 1321-1330.

http://www.biodiversity-science.net/CN/10.17520/biods.2017135

\begin{tabular}{|c|c|c|c|c|c|c|c|c|c|c|}
\hline \multirow{2}{*}{$\begin{array}{l}\text { 序号 } \\
\text { No. } \\
505\end{array}$} & \multicolumn{2}{|c|}{$\begin{array}{c}\text { 纲 } \\
\text { Class }\end{array}$} & \multicolumn{2}{|c|}{$\begin{array}{c}\text { 目 } \\
\text { Order }\end{array}$} & \multicolumn{2}{|r|}{$\begin{array}{c}\text { 科 } \\
\text { Family }\end{array}$} & \multicolumn{2}{|r|}{$\begin{array}{l}\text { 物种名 } \\
\text { Species }\end{array}$} & \multirow{2}{*}{$\begin{array}{l}\text { 分布型 } \\
\text { Chorotype } \\
\text { ha }\end{array}$} & \multirow[t]{2}{*}{$\begin{array}{c}\text { 备注 } \\
\text { Remarks }\end{array}$} \\
\hline & 爬行纲 & Reptilia & 有鳞目 & Squamata & 游蛇科 & Colubridae & 南峰锦蛇 & Elaphe hodgsonii & & \\
\hline 506 & 爬行纲 & Reptilia & 有鳞目 & Squamata & 游蛇科 & Colubridae & 玉斑锦蛇 & Elaphe mandarinus & sd & \\
\hline 507 & 爬行纲 & Reptilia & 有鳞目 & Squamata & 游蛇科 & Colubridae & 百花锦蛇 & Elaphe moellendorffi & $\mathrm{sb}$ & \\
\hline 508 & 爬行纲 & Reptilia & 有鳞目 & Squamata & 游蛇科 & Colubridae & 横斑锦蛇 & Elaphe perlacea & hc & \\
\hline 509 & 爬行纲 & Reptilia & 有鳞目 & Squamata & 游蛇科 & Colubridae & 紫灰锦蛇 & Elaphe porphyracen & we & \\
\hline 510 & 爬行纲 & Reptilia & 有鳞目 & Squamata & 游蛇科 & Colubridae & 绿锦蛇 & Elaphe prasina & wc & \\
\hline 511 & 爬行纲 & Reptilia & 有鳞目 & Squamata & 游蛇科 & Colubridae & 三索锦蛇 & Elaphe radiata & $\mathrm{wb}$ & \\
\hline 512 & 爬行纲 & Reptilia & 有鳞目 & Squamata & 游蛇科 & Colubridae & 红点锦蛇 & Elaphe rufodorsata & $\mathrm{eb}$ & \\
\hline 513 & 爬行纲 & Reptilia & 有鳞目 & Squamata & 游蛇科 & Colubridae & 棕黑锦蛇 & Elaphe schrenckii & eb & \\
\hline 514 & 爬行纲 & Reptilia & 有鳞目 & Squamata & 游蛇科 & Colubridae & 黑眉锦蛇 & Elaphe taeniura & we & \\
\hline 515 & 爬行纲 & Reptilia & 有鳞目 & Squamata & 游蛇科 & Colubridae & 黑斑水蛇 & Enhydris bennettii & $\mathrm{sa}$ & \\
\hline 516 & 爬行纲 & Reptilia & 有鳞目 & Squamata & 游蛇科 & Colubridae & 中国水蛇 & Enhydris chinensis & sc & \\
\hline 517 & 爬行纲 & Reptilia & 有鳞目 & Squamata & 游蛇科 & Colubridae & 铅色水蛇 & Enhydris plumbea & wc & \\
\hline 518 & 爬行纲 & Reptilia & 有鳞目 & Squamata & 游蛇科 & Colubridae & 滑鳞蛇 & Liopeltis frenatus & wa & \\
\hline 519 & 爬行纲 & Reptilia & 有鳞目 & Squamata & 游蛇科 & Colubridae & 白环蛇 & Lycodon aulicus & wa & \\
\hline 520 & 爬行纲 & Reptilia & 有鳞目 & Squamata & 游蛇科 & Colubridae & 双全白环蛇 & Lycodon fasciatus & we & \\
\hline 521 & 爬行纲 & Reptilia & 有鳞目 & Squamata & 游蛇科 & Colubridae & 老挝白环蛇 & Lycodon laoensis & wb & \\
\hline 522 & 爬行纲 & Reptilia & 有鳞目 & Squamata & 游蛇科 & Colubridae & 黑背白环蛇 & Lycodon ruhstrati & sd & \\
\hline 523 & 爬行纲 & Reptilia & 有鳞目 & Squamata & 游蛇科 & Colubridae & 细白环蛇 & Lycodon subcinctus & $\mathrm{wb}$ & \\
\hline 524 & 爬行纲 & Reptilia & 有鳞目 & Squamata & 游蛇科 & Colubridae & 颈棱蛇 & Macropisthodon rudis & $\operatorname{sh}$ & \\
\hline 525 & 爬行纲 & Reptilia & 有鳞目 & Squamata & 游蛇科 & Colubridae & 水游蛇 & Natrix natrix & ub & \\
\hline 526 & 爬行纲 & Reptilia & 有鳞目 & Squamata & 游蛇科 & Colubridae & 棋斑水游蛇 & Natrix tessellata & $\mathrm{dh}$ & \\
\hline
\end{tabular}


郜二虎, 何杰坤, 王志臣, 徐扬, 唐小平, 江海声. 全国陆生野生动物调查单元区划方案. 生物多样性, 2017, 25 (12): 1321-1330.

http://www.biodiversity-science.net/CN/10.17520/biods.2017135

\begin{tabular}{|c|c|c|c|c|c|c|c|c|c|c|}
\hline $\begin{array}{r}\text { 序号 } \\
\text { No. }\end{array}$ & \multicolumn{2}{|c|}{$\begin{array}{c}\text { 纲 } \\
\text { Class }\end{array}$} & \multicolumn{2}{|c|}{$\begin{array}{c}\text { 目 } \\
\text { Order }\end{array}$} & \multicolumn{2}{|r|}{$\begin{array}{c}\text { 科 } \\
\text { Family }\end{array}$} & \multicolumn{2}{|r|}{$\begin{array}{l}\text { 物种名 } \\
\text { Species }\end{array}$} & \multirow{2}{*}{$\begin{array}{l}\text { 分布型 } \\
\text { Chorotype } \\
\text { he }\end{array}$} & \multirow[t]{2}{*}{$\begin{array}{c}\text { 备注 } \\
\text { Remarks }\end{array}$} \\
\hline 527 & 爬行纲 & Reptilia & 有鳞目 & Squamata & 游蛇科 & Colubridae & 喜山小头蛇 & Oligodon albocinctus & & \\
\hline 528 & 爬行纲 & Reptilia & 有鳞目 & Squamata & 游蛇科 & Colubridae & 方花小头蛇 & Oligodon bellus & sc & \\
\hline 529 & 爬行纲 & Reptilia & 有鳞目 & Squamata & 游蛇科 & Colubridae & 菱斑小头蛇 & Oligodon catenata & wb & \\
\hline 530 & 爬行纲 & Reptilia & 有鳞目 & Squamata & 游蛇科 & Colubridae & 中国小头蛇 & Oligodon chinensis & $\mathrm{sc}$ & \\
\hline 531 & 爬行纲 & Reptilia & 有鳞目 & Squamata & 游蛇科 & Colubridae & 紫棕小头蛇 & Oligodon cinereus & wb & \\
\hline 532 & 爬行纲 & Reptilia & 有鳞目 & Squamata & 游蛇科 & Colubridae & 管状小头蛇 & Oligodon cyclurus & wa & 国内无分布 \\
\hline 533 & 爬行纲 & Reptilia & 有鳞目 & Squamata & 游蛇科 & Colubridae & 台湾小头蛇 & Oligodon formosanus & sc & \\
\hline 534 & 爬行纲 & Reptilia & 有鳞目 & Squamata & 游蛇科 & Colubridae & 昆明小头蛇 & Oligodon kunmingensis & si & 并入颈斑蛇 \\
\hline 535 & 爬行纲 & Reptilia & 有鳞目 & Squamata & 游蛇科 & Colubridae & 圆斑小头蛇 & Oligodon lacroixi & $\mathrm{sb}$ & \\
\hline 536 & 爬行纲 & Reptilia & 有鳞目 & Squamata & 游蛇科 & Colubridae & 龙胜小头蛇 & Oligodon lungshenensis & $\mathrm{sb}$ & \\
\hline 537 & 爬行纲 & Reptilia & 有鳞目 & Squamata & 游蛇科 & Colubridae & 黑带小头蛇 & Oligodon melanozonatus & he & \\
\hline 538 & 爬行纲 & Reptilia & 有鳞目 & Squamata & 游蛇科 & Colubridae & 横纹小头蛇 & Oligodon multizonatum & hc & \\
\hline 539 & 爬行纲 & Reptilia & 有鳞目 & Squamata & 游蛇科 & Colubridae & 宁陕小头蛇 & Oligodon ningshaanensis & $\mathrm{sm}$ & \\
\hline 540 & 爬行纲 & Reptilia & 有鳞目 & Squamata & 游蛇科 & Colubridae & 饰纹小头蛇 & Oligodon ornatus & si & \\
\hline 541 & 爬行纲 & Reptilia & 有鳞目 & Squamata & 游蛇科 & Colubridae & 山斑小头蛇 & Oligodon taeniatus & wb & \\
\hline 542 & 爬行纲 & Reptilia & 有鳞目 & Squamata & 游蛇科 & Colubridae & 香港后棱蛇 & Opisthotropis andersonii & $\mathrm{j}$ & \\
\hline 543 & 爬行纲 & Reptilia & 有鳞目 & Squamata & 游蛇科 & Colubridae & 横纹后棱蛇 & Opisthotropis balteata & wb & \\
\hline 544 & 爬行纲 & Reptilia & 有鳞目 & Squamata & 游蛇科 & Colubridae & 广西后棱蛇 & Opisthotropis guangxiensis & sg & \\
\hline 545 & 爬行纲 & Reptilia & 有鳞目 & Squamata & 游蛇科 & Colubridae & 沙坝后棱蛇 & Opisthotropis jacobi & sa & \\
\hline 546 & 爬行纲 & Reptilia & 有鳞目 & Squamata & 游蛇科 & Colubridae & 挂墩后棱蛇 & Opisthotropis kuatunensis & $\mathrm{sb}$ & \\
\hline 547 & 爬行纲 & Reptilia & 有鳞目 & Squamata & 游蛇科 & Colubridae & 侧条后棱蛇 & Opisthotropis lateralis & sc & \\
\hline 548 & 爬行纲 & Reptilia & 有鳞目 & Squamata & 游蛇科 & Colubridae & 山溪后棱蛇 & Opisthotropis latouchii & si & \\
\hline
\end{tabular}


郜二虎, 何杰坤, 王志臣, 徐扬, 唐小平, 江海声. 全国陆生野生动物调查单元区划方案. 生物多样性, 2017, 25 (12): 1321-1330.

http://www.biodiversity-science.net/CN/10.17520/biods.2017135

\begin{tabular}{|c|c|c|c|c|c|c|c|c|c|c|}
\hline \multirow{2}{*}{$\begin{array}{l}\text { 序号 } \\
\text { No. } \\
549\end{array}$} & \multicolumn{2}{|c|}{$\begin{array}{c}\text { 纲 } \\
\text { Class }\end{array}$} & \multicolumn{2}{|c|}{$\begin{array}{c}\text { 目 } \\
\text { Order }\end{array}$} & \multirow{2}{*}{\multicolumn{2}{|c|}{$\begin{array}{c}\text { 科 } \\
\text { Family } \\
\text { Colubridae }\end{array}$}} & \multirow{2}{*}{\multicolumn{2}{|c|}{$\begin{array}{c}\text { 物种名 } \\
\text { Species } \\
\text { Opisthotropis maxwelli }\end{array}$}} & \multirow{2}{*}{$\begin{array}{l}\begin{array}{c}\text { 分布型 } \\
\text { Chorotype }\end{array} \\
\text { si }\end{array}$} & \multirow[t]{2}{*}{$\begin{array}{c}\text { 备注 } \\
\text { Remarks }\end{array}$} \\
\hline & 爬行纲 & Reptilia & 有鳞目 & Squamata & & & & & & \\
\hline 550 & 爬行纲 & Reptilia & 有鳞目 & Squamata & 游蛇科 & Colubridae & 老挝后棱蛇 & Opisthotropis praemaxillaris & wb & \\
\hline 551 & 爬行纲 & Reptilia & 有鳞目 & Squamata & 游蛇科 & Colubridae & 平鳞钝头蛇 & Pareas boulengeri & sh & \\
\hline 552 & 爬行纲 & Reptilia & 有鳞目 & Squamata & 游蛇科 & Colubridae & 棱鳞钝头蛇 & Pareas carinatus & wa & \\
\hline 553 & 爬行纲 & Reptilia & 有鳞目 & Squamata & 游蛇科 & Colubridae & 缅甸针头蛇 & Pareas hamptoni & wb & \\
\hline 554 & 爬行纲 & Reptilia & 有鳞目 & Squamata & 游蛇科 & Colubridae & 横纹钝头蛇 & Pareas margaritophorus & wb & \\
\hline 555 & 爬行纲 & Reptilia & 有鳞目 & Squamata & 游蛇科 & Colubridae & 喜山钝头蛇 & Pareas monticola & he & \\
\hline 556 & 爬行纲 & Reptilia & 有鳞目 & Squamata & 游蛇科 & Colubridae & 福建钝头蛇 & Pareas stanleyi & si & \\
\hline 557 & 爬行纲 & Reptilia & 有鳞目 & Squamata & 游蛇科 & Colubridae & 颈斑蛇 & Plagiopholis blakewayi & he & \\
\hline 558 & 爬行纲 & Reptilia & 有鳞目 & Squamata & 游蛇科 & Colubridae & 缅甸颈斑蛇 & Plagiopholis nuchalis & wa & \\
\hline 559 & 爬行纲 & Reptilia & 有鳞目 & Squamata & 游蛇科 & Colubridae & 福建颈斑蛇 & Plagiopholis styani & si & \\
\hline 560 & 爬行纲 & Reptilia & 有鳞目 & Squamata & 游蛇科 & Colubridae & 云南颈斑蛇 & Plagiopholis unipostocularis & hc & \\
\hline 561 & 爬行纲 & Reptilia & 有鳞目 & Squamata & 游蛇科 & Colubridae & 紫沙蛇 & Psammodynastes pulverulentus & wc & \\
\hline 562 & 爬行纲 & Reptilia & 有鳞目 & Squamata & 游蛇科 & Colubridae & 花条蛇 & Psammophis lineolatus & $\mathrm{dc}$ & \\
\hline 563 & 爬行纲 & Reptilia & 有鳞目 & Squamata & 游蛇科 & Colubridae & 横纹斜鳞蛇 & Pseudoxenodon bambusicola & si & \\
\hline 564 & 爬行纲 & Reptilia & 有鳞目 & Squamata & 游蛇科 & Colubridae & 崇安斜鳞蛇 & Pseudoxenodon karlschmidti & sc & \\
\hline 565 & 爬行纲 & Reptilia & 有鳞目 & Squamata & 游蛇科 & Colubridae & 斜鳞蛇 & Pseudoxenodon macrops & we & \\
\hline 566 & 爬行纲 & Reptilia & 有鳞目 & Squamata & 游蛇科 & Colubridae & 花尾斜鳞蛇 & Pseudoxenodon stejnegeri & sh & \\
\hline 567 & 爬行纲 & Reptilia & 有鳞目 & Squamata & 游蛇科 & Colubridae & 灰鼠蛇 & Ptyas korros & wc & \\
\hline 568 & 爬行纲 & Reptilia & 有鳞目 & Squamata & 游蛇科 & Colubridae & 滑鼠蛇 & Ptyas mucosus & wc & \\
\hline 569 & 爬行纲 & Reptilia & 有鳞目 & Squamata & 游蛇科 & Colubridae & 海南颈槽蛇 & Rhabdophis adleri & $\mathrm{j}$ & \\
\hline 570 & 爬行纲 & Reptilia & 有鳞目 & Squamata & 游蛇科 & Colubridae & 喜山颈槽蛇 & Rhabdophis himalayanus & ha & \\
\hline
\end{tabular}


郜二虎, 何杰坤, 王志臣, 徐扬, 唐小平, 江海声. 全国陆生野生动物调查单元区划方案. 生物多样性, 2017, 25 (12): 1321-1330.

http://www.biodiversity-science.net/CN/10.17520/biods.2017135

\begin{tabular}{|c|c|c|c|c|c|c|c|c|c|c|}
\hline $\begin{array}{r}\text { 序号 } \\
\text { No. }\end{array}$ & \multicolumn{2}{|c|}{$\begin{array}{c}\text { 纲 } \\
\text { Class }\end{array}$} & \multicolumn{2}{|c|}{$\begin{array}{c}\text { 目 } \\
\text { Order }\end{array}$} & \multicolumn{2}{|r|}{$\begin{array}{c}\text { 科 } \\
\text { Family }\end{array}$} & \multicolumn{2}{|r|}{$\begin{array}{l}\text { 物种名 } \\
\text { Species }\end{array}$} & \multirow{2}{*}{$\begin{array}{l}\begin{array}{c}\text { 分布型 } \\
\text { Chorotype }\end{array} \\
\text { hc }\end{array}$} & \multirow[t]{2}{*}{$\begin{array}{c}\text { 备注 } \\
\text { Remarks }\end{array}$} \\
\hline 571 & 爬行纲 & Reptilia & 有鳞目 & Squamata & 游蛇科 & Colubridae & 缅甸颈槽蛇 & Rhabdophis leonardi & & \\
\hline 572 & 爬行纲 & Reptilia & 有鳞目 & Squamata & 游蛇科 & Colubridae & 黑纹颈槽蛇 & Rhabdophis nigrocinctus & wc & \\
\hline 573 & 爬行纲 & Reptilia & 有鳞目 & Squamata & 游蛇科 & Colubridae & 颈槽蛇 & Rhabdophis nuchalis & sd & \\
\hline 574 & 爬行纲 & Reptilia & 有鳞目 & Squamata & 游蛇科 & Colubridae & 九龙颈槽蛇 & Rhabdophis pentasupralabialis & hc & \\
\hline 575 & 爬行纲 & Reptilia & 有鳞目 & Squamata & 游蛇科 & Colubridae & 红脖颈槽蛇 & Rhabdophis subminiatus & we & \\
\hline 576 & 爬行纲 & Reptilia & 有鳞目 & Squamata & 游蛇科 & Colubridae & 台湾颈槽蛇 & Rhabdophis swinhonis & $\mathrm{j}$ & \\
\hline 577 & 爬行纲 & Reptilia & 有鳞目 & Squamata & 游蛇科 & Colubridae & 虎斑颈槽蛇 & Rhabdophis tigrinus & ea & \\
\hline 578 & 爬行纲 & Reptilia & 有鳞目 & Squamata & 游蛇科 & Colubridae & 黄腹杆蛇 & Rhabdops bicolor & he & \\
\hline 579 & 爬行纲 & Reptilia & 有鳞目 & Squamata & 游蛇科 & Colubridae & 尖喙蛇 & Rhynchophis boulengeri & $\mathrm{sc}$ & \\
\hline 580 & 爬行纲 & Reptilia & 有鳞目 & Squamata & 游蛇科 & Colubridae & 黑头剑蛇 & Sibynophis chinensis & $\mathrm{sd}$ & \\
\hline 581 & 爬行纲 & Reptilia & 有鳞目 & Squamata & 游蛇科 & Colubridae & 黑领剑蛇 & Sibynophis collaris & wc & \\
\hline 582 & 爬行纲 & Reptilia & 有鳞目 & Squamata & 游蛇科 & Colubridae & 环纹华游蛇 & Sinonatrix aequifasciata & $\mathrm{sb}$ & \\
\hline 583 & 爬行纲 & Reptilia & 有鳞目 & Squamata & 游蛇科 & Colubridae & 赤链华游蛇 & Sinonatrix annularis & $\mathrm{sc}$ & \\
\hline 584 & 爬行纲 & Reptilia & 有鳞目 & Squamata & 游蛇科 & Colubridae & 华游蛇 & Sinonatrix percarinata & sd & \\
\hline 585 & 爬行纲 & Reptilia & 有鳞目 & Squamata & 游蛇科 & Colubridae & 温泉蛇 & Thermophis baileyi & $\mathrm{hm}$ & \\
\hline 586 & 爬行纲 & Reptilia & 有鳞目 & Squamata & 游蛇科 & Colubridae & 山坭蛇 & Trachischium monticola & he & \\
\hline 587 & 爬行纲 & Reptilia & 有鳞目 & Squamata & 游蛇科 & Colubridae & 小头坭蛇 & Trachischium tenuiceps & he & \\
\hline 588 & 爬行纲 & Reptilia & 有鳞目 & Squamata & 游蛇科 & Colubridae & 渔游蛇 & Xenochrophis piscator & wc & \\
\hline 589 & 爬行纲 & Reptilia & 有鳞目 & Squamata & 游蛇科 & Colubridae & 黑网乌梢蛇 & Zaocys carinatus & wa & \\
\hline 590 & 爬行纲 & Reptilia & 有鳞目 & Squamata & 游蛇科 & Colubridae & 乌梢蛇 & Zaocys dhumnades & wc & \\
\hline 591 & 爬行纲 & Reptilia & 有鳞目 & Squamata & 游蛇科 & Colubridae & 黑线乌梢蛇 & Zaocys nigromarginatus & $\mathrm{hm}$ & \\
\hline 592 & 爬行纲 & Reptilia & 有鳞目 & Squamata & 眼镜蛇科 & Elapidae & 金环蛇 & Bungarus fasciatus & wc & \\
\hline
\end{tabular}


郜二虎, 何杰坤, 王志臣, 徐扬, 唐小平, 江海声. 全国陆生野生动物调查单元区划方案. 生物多样性, 2017, 25 (12): 1321-1330.

http://www.biodiversity-science.net/CN/10.17520/biods.2017135

\begin{tabular}{|c|c|c|c|c|c|c|c|c|c|c|}
\hline $\begin{array}{r}\text { 序号 } \\
\text { No. }\end{array}$ & \multicolumn{2}{|c|}{$\begin{array}{c}\text { 纲 } \\
\text { Class }\end{array}$} & \multicolumn{2}{|c|}{$\begin{array}{c}\text { 目 } \\
\text { Order }\end{array}$} & \multicolumn{2}{|r|}{$\begin{array}{c}\text { 科 } \\
\text { Family }\end{array}$} & \multicolumn{2}{|r|}{$\begin{array}{l}\text { 物种名 } \\
\text { Species }\end{array}$} & \multirow{2}{*}{$\begin{array}{l}\text { 分布型 } \\
\text { Chorotype } \\
\text { sc }\end{array}$} & \multirow[t]{2}{*}{$\begin{array}{c}\text { 备注 } \\
\text { Remarks }\end{array}$} \\
\hline 593 & 爬行纲 & Reptilia & 有鳞目 & Squamata & 眼镜蛇科 & Elapidae & 银环蛇 & Bungarus multicinctus & & \\
\hline 594 & 爬行纲 & Reptilia & 有鳞目 & Squamata & 眼镜蛇科 & Elapidae & 福建丽纹蛇 & Calliophis kelloggi & si & \\
\hline 595 & 爬行纲 & Reptilia & 有鳞目 & Squamata & 眼镜蛇科 & Elapidae & 丽纹蛇 & Calliophis macclellandi & wc & \\
\hline 596 & 爬行纲 & Reptilia & 有鳞目 & Squamata & 眼镜蛇科 & Elapidae & 眼镜蛇 & Naja naja & wc & 分为 2 种 \\
\hline 597 & 爬行纲 & Reptilia & 有鳞目 & Squamata & 眼镜蛇科 & Elapidae & 眼镜王蛇 & Ophiophagus hannah & wb & \\
\hline 598 & 爬行纲 & Reptilia & 有鳞目 & Squamata & 蝰科 & Viperidae & 白头蛙 & Azemiops feae & sc & \\
\hline 599 & 爬行纲 & Reptilia & 有鳞目 & Squamata & 蛏科 & Viperidae & 尖吻蝮 & Deinagkistrodon acutus & sc & \\
\hline 600 & 爬行纲 & Reptilia & 有鳞目 & Squamata & 蛏科 & Viperidae & 短尾蝮 & Gloydius brevicaudus & ea & \\
\hline 601 & 爬行纲 & Reptilia & 有鳞目 & Squamata & 蝰科 & Viperidae & 中介蝮 & Gloydius intermedius & de & \\
\hline 602 & 爬行纲 & Reptilia & 有鳞目 & Squamata & 蛏科 & Viperidae & 岩栖蝮 & Gloydius saxatilis & ka & \\
\hline 603 & 爬行纲 & Reptilia & 有鳞目 & Squamata & 蛏科 & Viperidae & 蛇岛蝮 & Gloydius shedaoensis & $\mathrm{k}$ & \\
\hline 604 & 爬行纲 & Reptilia & 有鳞目 & Squamata & 蝰科 & Viperidae & 高原蝮 & Gloydius strauchii & hc & \\
\hline 605 & 爬行纲 & Reptilia & 有鳞目 & Squamata & 蛏科 & Viperidae & 乌苏里蝮 & Gloydius ussuriensis & $\mathrm{m}$ & \\
\hline 606 & 爬行纲 & Reptilia & 有鳞目 & Squamata & 蛏科 & Viperidae & 茾山烙铁头蛇 & Ermia mangshanensis & sh & \\
\hline 607 & 爬行纲 & Reptilia & 有鳞目 & Squamata & 蛏科 & Viperidae & 山烙铁头蛇 & Ovophis monticola & wc & \\
\hline 608 & 爬行纲 & Reptilia & 有鳞目 & Squamata & 蛏科 & Viperidae & 察隅烙铁头蛇 & Ovophis zayuensis & he & \\
\hline 609 & 爬行纲 & Reptilia & 有鳞目 & Squamata & 蛏科 & Viperidae & 菜花原矛头蝮 & Protobothrops jerdonii & $\mathrm{hm}$ & \\
\hline 610 & 爬行纲 & Reptilia & 有鳞目 & Squamata & 蛏科 & Viperidae & 原矛头蝮 & Protobothrops mисrosquamatus & sd & \\
\hline 611 & 爬行纲 & Reptilia & 有鳞目 & Squamata & 蛏科 & Viperidae & 白唇竹叶青蛇 & Trimeresurus albolabris & wc & \\
\hline 612 & 爬行纲 & Reptilia & 有鳞目 & Squamata & 蛙科 & Viperidae & 台湾竹叶青蛇 & Trimeresurus gracilis & $\mathrm{j}$ & \\
\hline 613 & 爬行纲 & Reptilia & 有鳞目 & Squamata & 蝰科 & Viperidae & 墨脱竹叶青蛇 & Trimeresurus medoensis & he & \\
\hline 614 & 爬行纲 & Reptilia & 有鳞目 & Squamata & 蛀科 & Viperidae & 竹叶青蛇 & Trimeresurus stejnegeri & we & \\
\hline
\end{tabular}


郜二虎, 何杰坤, 王志臣, 徐扬, 唐小平, 江海声. 全国陆生野生动物调查单元区划方案. 生物多样性, 2017, 25 (12): 1321-1330.

http://www.biodiversity-science.net/CN/10.17520/biods.2017135

\begin{tabular}{|c|c|c|c|c|c|c|c|c|c|c|}
\hline $\begin{array}{r}\text { 序号 } \\
\text { No. }\end{array}$ & \multicolumn{2}{|c|}{$\begin{array}{c}\text { 纲 } \\
\text { Class }\end{array}$} & \multicolumn{2}{|c|}{$\begin{array}{c}\text { 目 } \\
\text { Order }\end{array}$} & \multicolumn{2}{|r|}{$\begin{array}{c}\text { 科 } \\
\text { Family }\end{array}$} & \multicolumn{2}{|r|}{$\begin{array}{l}\text { 物种名 } \\
\text { Species }\end{array}$} & \multirow{2}{*}{$\begin{array}{l}\begin{array}{c}\text { 分布型 } \\
\text { Chorotype }\end{array} \\
\text { ha }\end{array}$} & \multirow[t]{2}{*}{$\begin{array}{c}\text { 备注 } \\
\text { Remarks }\end{array}$} \\
\hline 615 & 爬行纲 & Reptilia & 有鳞目 & Squamata & 蛀科 & Viperidae & 西藏竹叶青蛇 & Trimeresurus tibetanus & & \\
\hline 616 & 爬行纲 & Reptilia & 有鳞目 & Squamata & 蛀科 & Viperidae & 乡城竹叶青蛇 & Trimeresurus xiangchengensis & hc & \\
\hline 617 & 爬行纲 & Reptilia & 有鳞目 & Squamata & 蛀科 & Viperidae & 云南竹叶青蛇 & Trimeresurus yunnanensis & $\mathrm{sc}$ & \\
\hline 618 & 爬行纲 & Reptilia & 有鳞目 & Squamata & 蝰科 & Viperidae & 极北蛏 & Vipera berus & $\mathrm{ub}$ & \\
\hline 619 & 爬行纲 & Reptilia & 有鳞目 & Squamata & 蛀科 & Viperidae & 圆斑蛏 & Vipera russellii & wb & \\
\hline 620 & 爬行纲 & Reptilia & 有鳞目 & Squamata & 蛀科 & Viperidae & 草原蛏 & Vipera ursinii & $\mathrm{dc}$ & \\
\hline 621 & 鸟纲 & Aves & 潜鸟目 & Gaviiformes & 潜鸟科 & Gaviidae & 黑喉潜鸟 & Gavia arctica & $\mathrm{ca}$ & \\
\hline 622 & 鸟纲 & Aves & 鸛鶶目 & Podicipediformes & 鸊鷵科 & Podicipedidae & 小辟鹰 & Tachybaptus ruficollis & we & \\
\hline 623 & 鸟纲 & Aves & 鷿鷹目 & Podicipediformes & 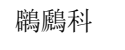 & Podicipedidae & 赤颈辟鹤 & Podiceps grisegena & ce & \\
\hline 624 & 鸟纲 & Aves & 钩嫄鳥目 & Podicipediformes & 碚鳥䳨科 & Podicipedidae & 角辟鹰 & Podiceps auritus & $\mathrm{cd}$ & \\
\hline 625 & 鸟纲 & Aves & 鸊鷵目 & Podicipediformes & 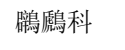 & Podicipedidae & 黑颈臂塬 & Podiceps nigricollis & $\mathrm{cd}$ & \\
\hline 626 & 鸟纲 & Aves & 獲形目 & Procellariiformes & 雘科 & Procellariidae & 楔尾獲 & Puffinus pacificus & awp & \\
\hline 627 & 鸟纲 & Aves & 鹈形目 & Pelecaniformes & 鹈胡科 & Pelecanidae & 白鹈胡 & Pelecanus onocrotalus & o & \\
\hline 628 & 鸟纲 & Aves & 鹈形目 & Pelecaniformes & 鹈鹕科 & Pelecanidae & 斑嘴鹈鹏 & Pelecanus philippensis & o & \\
\hline 629 & 鸟纲 & Aves & 鹈形目 & Pelecaniformes & 鲣鸟科 & Sulidae & 红脚鲣鸟 & Sula sula & aw & \\
\hline 630 & 鸟纲 & Aves & 鹈形目 & Pelecaniformes & 鲣鸟科 & Sulidae & 褐鲣鸟 & Sula leucogaster & $\mathrm{cwl}$ & \\
\hline 631 & 鸟纲 & Aves & 鹈形目 & Pelecaniformes & 扸鹚科 & Phalacrocoracidae & 黑颈鸬鹚 & Phalacrocorax niger & wa & \\
\hline 632 & 鸟纲 & Aves & 鹈形目 & Pelecaniformes & 军舰鸟科 & Fregatidae & 白腹军舰鸟 & Fregata andrewsi & uw & \\
\hline 633 & 鸟纲 & Aves & 鹈形目 & Pelecaniformes & 军舰鸟科 & Fregatidae & 黑腹军舰鸟 & Fregata minor & uw & \\
\hline 634 & 鸟纲 & Aves & 鹈形目 & Pelecaniformes & 军舰鸟科 & Fregatidae & 白斑军舰鸟 & Fregata ariel & $\mathrm{cw}$ & \\
\hline 635 & 鸟纲 & Aves & 鹳形目 & Ciconiiformes & 酔科 & Ardeidae & 大白鹗 & Egretta alba & o & \\
\hline 636 & 鸟纲 & Aves & 鹳形目 & Ciconiiformes & 鹭科 & Ardeidae & 黄嘴白䍄 & Egretta eulophotes & $\mathrm{m}$ & \\
\hline
\end{tabular}


郜二虎, 何杰坤, 王志臣, 徐扬, 唐小平, 江海声. 全国陆生野生动物调查单元区划方案. 生物多样性, 2017, 25 (12): 1321-1330.

http://www.biodiversity-science.net/CN/10.17520/biods.2017135

\begin{tabular}{|c|c|c|c|c|c|c|c|c|c|c|}
\hline \multirow{2}{*}{$\begin{array}{l}\text { 序号 } \\
\text { No. } \\
637\end{array}$} & \multicolumn{2}{|c|}{$\begin{array}{c}\text { 纲 } \\
\text { Class }\end{array}$} & \multicolumn{2}{|c|}{$\begin{array}{c}\text { 目 } \\
\text { Order }\end{array}$} & \multirow{2}{*}{\multicolumn{2}{|c|}{$\begin{array}{c}\text { 科 } \\
\text { Family } \\
\text { Ardeidae }\end{array}$}} & \multicolumn{2}{|r|}{$\begin{array}{l}\text { 物种名 } \\
\text { Species }\end{array}$} & \multirow{2}{*}{$\begin{array}{l}\begin{array}{c}\text { 分布型 } \\
\text { Chorotype }\end{array} \\
\text { wb }\end{array}$} & \multirow[t]{2}{*}{$\begin{array}{c}\text { 备注 } \\
\text { Remarks }\end{array}$} \\
\hline & 鸟纲 & Aves & 鹳形目 & Ciconiiformes & & & 岩鹭 & Egretta sacra & & \\
\hline 638 & 鸟纲 & Aves & 鹳形目 & Ciconiiformes & 酔科 & Ardeidae & 夜鹭 & Nycticorax nycticorax & $\mathrm{o} 2$ & \\
\hline 639 & 鸟纲 & Aves & 鹳形目 & Ciconiiformes & 笉科 & Ardeidae & 海南鳽 & Gorsachius magnificus & Sc & \\
\hline 640 & 鸟纲 & Aves & 鹳形目 & Ciconiiformes & 酔科 & Ardeidae & 黑冠鳽 & Gorsachius melanolophus & wa & \\
\hline 641 & 鸟纲 & Aves & 鹳形目 & Ciconiiformes & 鸹科 & Ardeidae & 小苇鳽 & Ixobrychus minutus & 03 & \\
\hline 642 & 鸟纲 & Aves & 鹳形目 & Ciconiiformes & 笉科 & Ardeidae & 大麻鳽 & Botaurus stellaris & uc & \\
\hline 643 & 鸟纲 & Aves & 鹳形目 & Ciconiiformes & 鹳科 & Ciconiidae & 彩鹳 & Mycteria leucocephala & wc & \\
\hline 644 & 鸟纲 & Aves & 鹳形目 & Ciconiiformes & 鹳科 & Ciconiidae & 黑鹳 & Ciconia nigra & uf & \\
\hline 645 & 鸟纲 & Aves & 鹳形目 & Ciconiiformes & 鹳科 & Ciconiidae & 白鹳 & Ciconia ciconia & uc & \\
\hline 646 & 鸟纲 & Aves & 鹳形目 & Ciconiiformes & 鹳科 & Ciconiidae & 秃鹳 & Leptoptilos javanicus & wc & \\
\hline 647 & 鸟纲 & Aves & 鹳形目 & Ciconiiformes & 鰢科 & Threskiornithidae & 白肩黑蟷鸟 & Pseudibis davisoni & wa & \\
\hline 648 & 鸟纲 & Aves & 鹳形目 & Ciconiiformes & 器科 & Threskiornithidae & 朱器 & Nipponia nippon & ed & \\
\hline 649 & 鸟纲 & Aves & 鹳形目 & Ciconiiformes & 噮科 & Threskiornithidae & 白琵瞪 & Platalea leucorodia & o & \\
\hline 650 & 鸟纲 & Aves & 雁形目 & Anseriformes & 鸭科 & Anatidae & 栗树鸭 & Dendrocygna javanica & wd & \\
\hline 651 & 鸟纲 & Aves & 雁形目 & Anseriformes & 鸭科 & Anatidae & 疮鼻天鹅 & Cygnus olor & ud & \\
\hline 652 & 鸟纲 & Aves & 雁形目 & Anseriformes & 鸭科 & Anatidae & 大天鹅 & Cygnus cygnus & ce & \\
\hline 653 & 鸟纲 & Aves & 雁形目 & Anseriformes & 鸭科 & Anatidae & 鸿雁 & Anser cygnoides & $\mathrm{m}$ & \\
\hline 654 & 鸟纲 & Aves & 雁形目 & Anseriformes & 鸭科 & Anatidae & 小白额雁 & Anser erythropus & ua & \\
\hline 655 & 鸟纲 & Aves & 雁形目 & Anseriformes & 鸭科 & Anatidae & 灰雁 & Anser anser & uc & \\
\hline 656 & 鸟纲 & Aves & 雁形目 & Anseriformes & 鸭科 & Anatidae & 斑头雁 & Anser indicus & $\mathrm{p}$ & \\
\hline 657 & 鸟纲 & Aves & 雁形目 & Anseriformes & 鸭科 & Anatidae & 赤麻鸭 & Tadorna ferruginea & uf & \\
\hline 658 & 鸟纲 & Aves & 雁形目 & Anseriformes & 鸭科 & Anatidae & 翅鼻麻鸭 & Tadorna tadorna & uf & \\
\hline
\end{tabular}


郜二虎, 何杰坤, 王志臣, 徐扬, 唐小平, 江海声. 全国陆生野生动物调查单元区划方案. 生物多样性, 2017, 25 (12): 1321-1330,

http://www.biodiversity-science.net/CN/10.17520/biods.2017135

\begin{tabular}{|c|c|c|c|c|c|c|c|c|c|c|}
\hline \multirow{2}{*}{$\begin{array}{l}\text { 序号 } \\
\text { No. } \\
659\end{array}$} & \multicolumn{2}{|c|}{$\begin{array}{c}\text { 纲 } \\
\text { Class }\end{array}$} & \multicolumn{2}{|c|}{$\begin{array}{c}\text { 目 } \\
\text { Order }\end{array}$} & \multirow{2}{*}{\multicolumn{2}{|c|}{$\begin{array}{c}\text { 科 } \\
\text { Family } \\
\text { Anatidae }\end{array}$}} & \multicolumn{2}{|r|}{$\begin{array}{l}\text { 物种名 } \\
\text { Species }\end{array}$} & \multirow{2}{*}{$\begin{array}{l}\text { 分布型 } \\
\text { Chorotype }\end{array}$} & \multirow[t]{2}{*}{$\begin{array}{c}\text { 备注 } \\
\text { Remarks }\end{array}$} \\
\hline & 鸟纲 & Aves & 雁形目 & Anseriformes & & & 棉嵬 & Nettapus coromandelianus & & \\
\hline 660 & 鸟纲 & Aves & 雁形目 & Anseriformes & 鸭科 & Anatidae & 鹗茑 & Aix galericulata & eh & \\
\hline 661 & 鸟纲 & Aves & 雁形目 & Anseriformes & 鸭科 & Anatidae & 赤颈鸭 & Anas penelope & ce & \\
\hline 662 & 鸟纲 & Aves & 雁形目 & Anseriformes & 鸭科 & Anatidae & 罗纹鸭 & Anas falcata & $\mathrm{m}$ & \\
\hline 663 & 鸟纲 & Aves & 雁形目 & Anseriformes & 鸭科 & Anatidae & 赤膀鸭 & Anas strepera & uf & \\
\hline 664 & 鸟纲 & Aves & 雁形目 & Anseriformes & 鸭科 & Anatidae & 绿翅鸭 & Anas crecca & ce & \\
\hline 665 & 鸟纲 & Aves & 雁形目 & Anseriformes & 鸭科 & Anatidae & 绿头鸭 & Anas platyrhynchos & $\mathrm{cf}$ & \\
\hline 666 & 鸟纲 & Aves & 雁形目 & Anseriformes & 鸭科 & Anatidae & 斑嘴鸭 & Anas poecilorhyncha & we & \\
\hline 667 & 鸟纲 & Aves & 雁形目 & Anseriformes & 鸭科 & Anatidae & 针尾鸭 & Anas acuta & ce & \\
\hline 668 & 鸟纲 & Aves & 雁形目 & Anseriformes & 鸭科 & Anatidae & 白眉鸭 & Anas querquedula & uf & \\
\hline 669 & 鸟纲 & Aves & 雁形目 & Anseriformes & 鸭科 & Anatidae & 琵嘴鸭 & Anas clypeata & $\mathrm{cf}$ & \\
\hline 670 & 鸟纲 & Aves & 雁形目 & Anseriformes & 鸭科 & Anatidae & 赤嘴潜鸭 & Netta rufina & o3 & \\
\hline 671 & 鸟纲 & Aves & 雁形目 & Anseriformes & 鸭科 & Anatidae & 红头潜鸭 & Aythya ferina & $\mathrm{cf}$ & \\
\hline 672 & 鸟纲 & Aves & 雁形目 & Anseriformes & 鸭科 & Anatidae & 青头潜鸭 & Aythya baeri & $\mathrm{ma}$ & \\
\hline 673 & 鸟纲 & Aves & 雁形目 & Anseriformes & 鸭科 & Anatidae & 白眼潜鸭 & Aythya nyroca & o3 & \\
\hline 674 & 鸟纲 & Aves & 雁形目 & Anseriformes & 鸭科 & Anatidae & 凤头潜鸭 & Aythya fuligula & uf & \\
\hline 675 & 鸟纲 & Aves & 雁形目 & Anseriformes & 鸭科 & Anatidae & 鹊鸭 & Bucephala clangula & $\mathrm{cb}$ & \\
\hline 676 & 鸟纲 & Aves & 雁形目 & Anseriformes & 鸭科 & Anatidae & 斑头秋沙鸭 & Mergus albellus & uc & \\
\hline 677 & 鸟纲 & Aves & 雁形目 & Anseriformes & 鸭科 & Anatidae & 红胸秋沙鸭 & Mergus serrator & $\mathrm{ca}$ & \\
\hline 678 & 鸟纲 & Aves & 雁形目 & Anseriformes & 鸭科 & Anatidae & 普通秋沙鸭 & Mergus merganser & $\mathrm{cb}$ & \\
\hline 679 & 鸟纲 & Aves & 雁形目 & Anseriformes & 鸭科 & Anatidae & 中华秋沙鸭 & Mergus squamatus & $\mathrm{ma}$ & \\
\hline 680 & 鸟纲 & Aves & 雁形目 & Anseriformes & 鸭科 & Anatidae & 白头硬尾鸭 & Oxyura leucocephala & 03 & \\
\hline
\end{tabular}


郜二虎, 何杰坤, 王志臣, 徐扬, 唐小平, 江海声. 全国陆生野生动物调查单元区划方案. 生物多样性, 2017, 25 (12): 1321-1330.

http://www.biodiversity-science.net/CN/10.17520/biods.2017135

\begin{tabular}{|c|c|c|c|c|c|c|c|c|c|c|}
\hline \multirow{2}{*}{$\begin{array}{l}\text { 序号 } \\
\text { No. } \\
681\end{array}$} & \multicolumn{2}{|c|}{$\begin{array}{c}\text { 纲 } \\
\text { Class }\end{array}$} & \multicolumn{2}{|c|}{$\begin{array}{c}\text { 目 } \\
\text { Order }\end{array}$} & \multicolumn{2}{|r|}{$\begin{array}{c}\text { 科 } \\
\text { Family }\end{array}$} & \multicolumn{2}{|r|}{$\begin{array}{l}\text { 物种名 } \\
\text { Species }\end{array}$} & \multirow{2}{*}{$\begin{array}{l}\begin{array}{c}\text { 分布型 } \\
\text { Chorotype }\end{array} \\
\mathrm{cd}\end{array}$} & \multirow[t]{2}{*}{$\begin{array}{c}\text { 备注 } \\
\text { Remarks }\end{array}$} \\
\hline & 鸟纲 & Aves & 隼形目 & Falconiformes & 鸣科 & Pandionidae & 哿 & Pandion haliaetus & & \\
\hline 682 & 鸟纲 & Aves & 隼形目 & Falconiformes & 鹰科 & Accipitridae & 褐冠鹃隼 & Aviceda jerdoni & wa & \\
\hline 683 & 鸟纲 & Aves & 隼形目 & Falconiformes & 鹰科 & Accipitridae & 黑冠鹃隼 & Aviceda leuphotes & wa & \\
\hline 684 & 鸟纲 & Aves & 隼形目 & Falconiformes & 鹰科 & Accipitridae & 凤头蜂鹰 & Pernis ptilorhyncus & we & \\
\hline 685 & 鸟纲 & Aves & 隼形目 & Falconiformes & 鹰科 & Accipitridae & 白腹海雕 & Haliaeetus leucogaster & we & \\
\hline 686 & 鸟纲 & Aves & 隼形目 & Falconiformes & 鹰科 & Accipitridae & 玉带海雕 & Haliaeetus leucoryphus & $\mathrm{db}$ & \\
\hline 687 & 鸟纲 & Aves & 隼形目 & Falconiformes & 鹰科 & Accipitridae & 胡兀鹑 & Gypaetus barbatus & 03 & \\
\hline 688 & 鸟纲 & Aves & 隼形目 & Falconiformes & 鹰科 & Accipitridae & 白背兀鹑 & Gyps bengalensis & wa & \\
\hline 689 & 鸟纲 & Aves & 隼形目 & Falconiformes & 鹰科 & Accipitridae & 高山兀鹑 & Gyps himalayensis & o3 & \\
\hline 690 & 鸟纲 & Aves & 隼形目 & Falconiformes & 鹰科 & Accipitridae & 秃鹑 & Aegypius monachus & 03 & \\
\hline 691 & 鸟纲 & Aves & 隼形目 & Falconiformes & 鹰科 & Accipitridae & 黑兀鹑 & Sarcogyps calvus & wb & \\
\hline 692 & 鸟纲 & Aves & 隼形目 & Falconiformes & 鹰科 & Accipitridae & 蛇雕 & Spilornis cheela & wc & \\
\hline 693 & 鸟纲 & Aves & 隼形目 & Falconiformes & 鹰科 & Accipitridae & 白头鹞 & Circus aeruginosus & 03 & \\
\hline 694 & 鸟纲 & Aves & 隼形目 & Falconiformes & 鹰科 & Accipitridae & 白尾䍃 & Circus cyaneus & $\mathrm{cd}$ & \\
\hline 695 & 鸟纲 & Aves & 隼形目 & Falconiformes & 鹰科 & Accipitridae & 草原䍃 & Circus macrourus & $\mathrm{dp}$ & \\
\hline 696 & 鸟纲 & Aves & 隼形目 & Falconiformes & 鹰科 & Accipitridae & 乌灰鹞 & Circus pygargus & $\mathrm{dp}$ & \\
\hline 697 & 鸟纲 & Aves & 隼形目 & Falconiformes & 鹰科 & Accipitridae & 凤头鹰 & Accipiter trivirgatus & wc & \\
\hline 698 & 鸟纲 & Aves & 隼形目 & Falconiformes & 鹰科 & Accipitridae & 褐耳鹰 & Accipiter badius & wb & \\
\hline 699 & 鸟纲 & Aves & 隼形目 & Falconiformes & 鹰科 & Accipitridae & 松雀鹰 & Accipiter virgatus & we & \\
\hline 700 & 鸟纲 & Aves & 隼形目 & Falconiformes & 鹰科 & Accipitridae & 雀鹰 & Accipiter nisus & ue & \\
\hline 701 & 鸟纲 & Aves & 隼形目 & Falconiformes & 鹰科 & Accipitridae & 苍鹰 & Accipiter gentilis & $\mathrm{cc}$ & \\
\hline 702 & 鸟纲 & Aves & 隼形目 & Falconiformes & 鹰科 & Accipitridae & 白眼鵟鹰 & Butastur teesa & wa & \\
\hline
\end{tabular}


郜二虎, 何杰坤, 王志臣, 徐扬, 唐小平, 江海声. 全国陆生野生动物调查单元区划方案. 生物多样性, 2017, 25 (12): 1321-1330.

http://www.biodiversity-science.net/CN/10.17520/biods.2017135

\begin{tabular}{|c|c|c|c|c|c|c|c|c|c|c|}
\hline \multirow{2}{*}{$\begin{array}{l}\text { 序号 } \\
\text { No. } \\
703\end{array}$} & \multicolumn{2}{|c|}{$\begin{array}{c}\text { 纲 } \\
\text { Class }\end{array}$} & \multicolumn{2}{|c|}{$\begin{array}{c}\text { 目 } \\
\text { Order }\end{array}$} & \multirow{2}{*}{\multicolumn{2}{|c|}{$\begin{array}{c}\text { 科 } \\
\text { Family } \\
\text { Accipitridae }\end{array}$}} & \multicolumn{2}{|r|}{$\begin{array}{l}\text { 物种名 } \\
\text { Species }\end{array}$} & \multirow{2}{*}{$\begin{array}{l}\begin{array}{c}\text { 分布型 } \\
\text { Chorotype }\end{array} \\
\mathrm{mb}\end{array}$} & \multirow[t]{2}{*}{$\begin{array}{c}\text { 备注 } \\
\text { Remarks }\end{array}$} \\
\hline & 鸟纲 & Aves & 隼形目 & Falconiformes & & & 灰脸鵟鹰 & Butastur indicus & & \\
\hline 704 & 鸟纲 & Aves & 隼形目 & Falconiformes & 鹰科 & Accipitridae & 普通鵟 & Buteo buteo & ud & \\
\hline 705 & 鸟纲 & Aves & 隼形目 & Falconiformes & 鹰科 & Accipitridae & 大鵟 & Buteo hemilasius & $\mathrm{df}$ & \\
\hline 706 & 鸟纲 & Aves & 隼形目 & Falconiformes & 鹰科 & Accipitridae & 草原雕 & Aquila nipalensis & da & \\
\hline 707 & 鸟纲 & Aves & 隼形目 & Falconiformes & 鹰科 & Accipitridae & 白肩雕 & Aquila heliaca & 03 & \\
\hline 708 & 鸟纲 & Aves & 隼形目 & Falconiformes & 鹰科 & Accipitridae & 金雕 & Aquila chrysaetos & ce & \\
\hline 709 & 鸟纲 & Aves & 隼形目 & Falconiformes & 鹰科 & Accipitridae & 白腹隼雕 & Aquila fasciata & we & \\
\hline 710 & 鸟纲 & Aves & 隼形目 & Falconiformes & 鹰科 & Accipitridae & 棕腹隼雕 & Hieraaetus kienerii & wa & \\
\hline 711 & 鸟纲 & Aves & 隼形目 & Falconiformes & 鹰科 & Accipitridae & 鹰雕 & Spizaetus nipalensis & wc & \\
\hline 712 & 鸟纲 & Aves & 隼形目 & Falconiformes & 隼科 & Falconidae & 白腿小隼 & Microhierax melanoleucos & wc & \\
\hline 713 & 鸟纲 & Aves & 隼形目 & Falconiformes & 隼科 & Falconidae & 黄爪隼 & Falco naumanni & ue & \\
\hline 714 & 鸟纲 & Aves & 隼形目 & Falconiformes & 隼科 & Falconidae & 红隼 & Falco tinnunculus & o1 & \\
\hline 715 & 鸟纲 & Aves & 隼形目 & Falconiformes & 隼科 & Falconidae & 红脚隼 & Falco amurensis & ud & \\
\hline 716 & 鸟纲 & Aves & 隼形目 & Falconiformes & 隼科 & Falconidae & 燕隼 & Falco subbuteo & ug & \\
\hline 717 & 鸟纲 & Aves & 隼形目 & Falconiformes & 隼科 & Falconidae & 猛隼 & Falco severus & wb & \\
\hline 718 & 鸟纲 & Aves & 隼形目 & Falconiformes & 隼科 & Falconidae & 猎隼 & Falco cherrug & $\mathrm{ca}$ & \\
\hline 719 & 鸟纲 & Aves & 鸡形目 & Galliformes & 松鸡科 & Tetraonidae & 镰翅鸡 & Dendragapus falcipennis & $\mathrm{ma}$ & \\
\hline 720 & 鸟纲 & Aves & 鸡形目 & Galliformes & 松鸡科 & Tetraonidae & 岩雷鸟 & Lagopus mutus & $\mathrm{ca}$ & \\
\hline 721 & 鸟纲 & Aves & 鸡形目 & Galliformes & 松鸡科 & Tetraonidae & 黑琴鸡 & Lyrurus tetrix & uc & \\
\hline 722 & 鸟纲 & Aves & 鸡形目 & Galliformes & 松鸡科 & Tetraonidae & 松鸡 & Tetrao urogallus & uc & \\
\hline 723 & 鸟纲 & Aves & 鸡形目 & Galliformes & 松鸡科 & Tetraonidae & 黑嘴松鸡 & Tetrao parvirostris & $\mathrm{ma}$ & \\
\hline 724 & 鸟纲 & Aves & 鸡形目 & Galliformes & 松鸡科 & Tetraonidae & 花尾榛鸡 & Bonasa bonasia & ua & \\
\hline
\end{tabular}


郜二虎, 何杰坤, 王志臣, 徐扬, 唐小平, 江海声. 全国陆生野生动物调查单元区划方案. 生物多样性, 2017, 25 (12): 1321-1330.

http://www.biodiversity-science.net/CN/10.17520/biods.2017135

\begin{tabular}{|c|c|c|c|c|c|c|c|c|c|c|}
\hline \multirow{2}{*}{$\begin{array}{l}\text { 序号 } \\
\text { No. } \\
725\end{array}$} & \multicolumn{2}{|c|}{$\begin{array}{c}\text { 纲 } \\
\text { Class }\end{array}$} & \multicolumn{2}{|c|}{$\begin{array}{c}\text { 目 } \\
\text { Order }\end{array}$} & \multirow{2}{*}{\multicolumn{2}{|c|}{$\begin{array}{c}\text { 科 } \\
\text { Family } \\
\text { Tetraonidae }\end{array}$}} & \multicolumn{2}{|r|}{$\begin{array}{l}\text { 物种名 } \\
\text { Species }\end{array}$} & \multirow{2}{*}{$\begin{array}{l}\begin{array}{c}\text { 分布型 } \\
\text { Chorotype }\end{array} \\
\text { hc }\end{array}$} & \multirow[t]{2}{*}{$\begin{array}{c}\text { 备注 } \\
\text { Remarks }\end{array}$} \\
\hline & 鸟纲 & Aves & 鸡形目 & Galliformes & & & 斑尾榛鸡 & Bonasa sewerzowi & & \\
\hline 726 & 鸟纲 & Aves & 鸡形目 & Galliformes & 雉科 & Phasianidae & 雪鹑 & Lerwa lerwa & $\mathrm{hm}$ & \\
\hline 727 & 鸟纲 & Aves & 鸡形目 & Galliformes & 雉科 & Phasianidae & 红喉雉敦 & Tetraophasis obscurus & $\mathrm{hc}$ & \\
\hline 728 & 鸟纲 & Aves & 鸡形目 & Galliformes & 雉科 & Phasianidae & 黄喉雉敦 & Tetraophasis szechenyii & hc & \\
\hline 729 & 鸟纲 & Aves & 鸡形目 & Galliformes & 雉科 & Phasianidae & 藏雪鸡 & Tetraogallus tibetanus & pa & \\
\hline 730 & 鸟纲 & Aves & 鸡形目 & Galliformes & 雉科 & Phasianidae & 阿尔泰雪鸡 & Tetraogallus altaicus & ua & \\
\hline 731 & 鸟纲 & Aves & 鸡形目 & Galliformes & 雉科 & Phasianidae & 暗腹雪鸡 & Tetraogallus himalayensis & pg & \\
\hline 732 & 鸟纲 & Aves & 鸡形目 & Galliformes & 雉科 & Phasianidae & 大石鸡 & Alectoris magna & $\mathrm{pf}$ & \\
\hline 733 & 鸟纲 & Aves & 鸡形目 & Galliformes & 雉科 & Phasianidae & 石鸡 & Alectoris chukar & de & \\
\hline 734 & 鸟纲 & Aves & 鸡形目 & Galliformes & 雉科 & Phasianidae & 中华麅鸪 & Francolinus pintadeanus & wc & \\
\hline 735 & 鸟纲 & Aves & 鸡形目 & Galliformes & 雉科 & Phasianidae & 灰山㝇 & Perdix perdix & ud & \\
\hline 736 & 鸟纲 & Aves & 鸡形目 & Galliformes & 雉科 & Phasianidae & 斑翅山敦 & Perdix dauuricae & de & \\
\hline 737 & 鸟纲 & Aves & 鸡形目 & Galliformes & 雉科 & Phasianidae & 高原山敦 & Perdix hodgsoniae & $\mathrm{hm}$ & \\
\hline 738 & 鸟纲 & Aves & 鸡形目 & Galliformes & 雉科 & Phasianidae & 鹤敦 & Coturnix coturnix & o & \\
\hline 739 & 鸟纲 & Aves & 鸡形目 & Galliformes & 雉科 & Phasianidae & 蓝胸鹑 & Coturnix chinensis & wb & \\
\hline 740 & 鸟纲 & Aves & 鸡形目 & Galliformes & 雉科 & Phasianidae & 环颈山府鸪 & Arborophila torqueola & wc & \\
\hline 741 & 鸟纲 & Aves & 鸡形目 & Galliformes & 雉科 & Phasianidae & 四川山麅鸪 & Arborophila rufipectus & hc & \\
\hline 742 & 鸟纲 & Aves & 鸡形目 & Galliformes & 雉科 & Phasianidae & 白眉山麅鸪 & Arborophila gingica & $\mathrm{sc}$ & \\
\hline 743 & 鸟纲 & Aves & 鸡形目 & Galliformes & 雉科 & Phasianidae & 白㚘页山麅鸪 & Arborophila atrogularis & wa & \\
\hline 744 & 鸟纲 & Aves & 鸡形目 & Galliformes & 雉科 & Phasianidae & 海南山麅鸪 & Arborophila ardens & $\mathrm{j}$ & \\
\hline 745 & 鸟纲 & Aves & 鸡形目 & Galliformes & 雉科 & Phasianidae & 褐胸山麅鸪 & Arborophila brunneopectus & wa & \\
\hline 746 & 鸟纲 & Aves & 鸡形目 & Galliformes & 雉科 & Phasianidae & 绿脚山麅扸 & Arborophila chloropus & wa & \\
\hline
\end{tabular}


郜二虎, 何杰坤, 王志臣, 徐扬, 唐小平, 江海声. 全国陆生野生动物调查单元区划方案. 生物多样性, 2017, 25 (12): 1321-1330.

http://www.biodiversity-science.net/CN/10.17520/biods.2017135

\begin{tabular}{|c|c|c|c|c|c|c|c|c|c|c|}
\hline \multirow{2}{*}{$\begin{array}{l}\text { 序号 } \\
\text { No. } \\
747\end{array}$} & \multicolumn{2}{|c|}{$\begin{array}{c}\text { 纲 } \\
\text { Class }\end{array}$} & \multicolumn{2}{|c|}{$\begin{array}{c}\text { 目 } \\
\text { Order }\end{array}$} & \multicolumn{2}{|r|}{$\begin{array}{c}\text { 科 } \\
\text { Family }\end{array}$} & \multicolumn{2}{|r|}{$\begin{array}{l}\text { 物种名 } \\
\text { Species }\end{array}$} & \multirow{2}{*}{$\begin{array}{l}\text { 分布型 } \\
\text { Chorotype }\end{array}$} & \multirow[t]{2}{*}{$\begin{array}{c}\text { 备注 } \\
\text { Remarks }\end{array}$} \\
\hline & 鸟纲 & Aves & 鸡形目 & Galliformes & 雉科 & Phasianidae & 灰胸竹鸡 & Bambusicola thoracica & & \\
\hline 748 & 鸟纲 & Aves & 鸡形目 & Galliformes & 雉科 & Phasianidae & 血雉 & Ithaginis cruentes & $\mathrm{hm}$ & \\
\hline 749 & 鸟纲 & Aves & 鸡形目 & Galliformes & 雉科 & Phasianidae & 黑头角雉 & Tragopan melanocephalus & ha & \\
\hline 750 & 鸟纲 & Aves & 鸡形目 & Galliformes & 雉科 & Phasianidae & 红胸角雉 & Tragopan satyra & ha & \\
\hline 751 & 鸟纲 & Aves & 鸡形目 & Galliformes & 雉科 & Phasianidae & 灰腹角雉 & Tragopan blythii & he & \\
\hline 752 & 鸟纲 & Aves & 鸡形目 & Galliformes & 雉科 & Phasianidae & 红腹角雉 & Tragopan temminckii & $\mathrm{hm}$ & \\
\hline 753 & 鸟纲 & Aves & 鸡形目 & Galliformes & 雉科 & Phasianidae & 黄腹角雉 & Tragopan caboti & sc & \\
\hline 754 & 鸟纲 & Aves & 鸡形目 & Galliformes & 雉科 & Phasianidae & 勺鸡 & Pucrasia macrolopha & si & \\
\hline 755 & 鸟纲 & Aves & 鸡形目 & Galliformes & 雉科 & Phasianidae & 棕尾虹雉 & Lophophorus impejanus & ha & \\
\hline 756 & 鸟纲 & Aves & 鸡形目 & Galliformes & 雉科 & Phasianidae & 白尾梢虹雉 & Lophophorus sclateri & he & \\
\hline 757 & 鸟纲 & Aves & 鸡形目 & Galliformes & 雉科 & Phasianidae & 绿尾虹雉 & Lophophorus Ihuysii & hc & \\
\hline 758 & 鸟纲 & Aves & 鸡形目 & Galliformes & 雉科 & Phasianidae & 原鸡 & Gallus gallus & wa & \\
\hline 759 & 鸟纲 & Aves & 鸡形目 & Galliformes & 雉科 & Phasianidae & 黑䣱 & Lophura leucomelanaos & wa & \\
\hline 760 & 鸟纲 & Aves & 鸡形目 & Galliformes & 雉科 & Phasianidae & 白鹏 & Lophura nycthemera & wc & \\
\hline 761 & 鸟纲 & Aves & 鸡形目 & Galliformes & 雉科 & Phasianidae & 白马鸡 & Crossoptilon crossoptilon & $\mathrm{hm}$ & \\
\hline 762 & 鸟纲 & Aves & 鸡形目 & Galliformes & 雉科 & Phasianidae & 藏马鸡 & Crossoptilon harmani & $\mathrm{hm}$ & \\
\hline 763 & 鸟纲 & Aves & 鸡形目 & Galliformes & 雉科 & Phasianidae & 蓝马鸡 & Crossoptilon auritum & $\mathrm{pf}$ & \\
\hline 764 & 鸟纲 & Aves & 鸡形目 & Galliformes & 雉科 & Phasianidae & 褐马鸡 & Crossoptilon mantchuricum & $\mathrm{b}$ & \\
\hline 765 & 鸟纲 & Aves & 鸡形目 & Galliformes & 雉科 & Phasianidae & 白颈长尾雉 & Syrmaticus ellioti & se & \\
\hline 766 & 鸟纲 & Aves & 鸡形目 & Galliformes & 雉科 & Phasianidae & 黑颈长尾雉 & Syrmaticus humiae & wa & \\
\hline 767 & 鸟纲 & Aves & 鸡形目 & Galliformes & 雉科 & Phasianidae & 白冠长尾雉 & Syrmaticus reevesii & $\mathrm{sm}$ & \\
\hline 768 & 鸟纲 & Aves & 鸡形目 & Galliformes & 雉科 & Phasianidae & 环颈雉 & Phasianus colchicus & o & \\
\hline
\end{tabular}


郜二虎, 何杰坤, 王志臣, 徐扬, 唐小平, 江海声. 全国陆生野生动物调查单元区划方案. 生物多样性, 2017, 25 (12): 1321-1330,

http://www.biodiversity-science.net/CN/10.17520/biods.2017135

\begin{tabular}{|c|c|c|c|c|c|c|c|c|c|c|}
\hline \multirow{2}{*}{$\begin{array}{l}\text { 序号 } \\
\text { No. } \\
769\end{array}$} & \multicolumn{2}{|c|}{$\begin{array}{c}\text { 纲 } \\
\text { Class }\end{array}$} & \multicolumn{2}{|c|}{$\begin{array}{c}\text { 目 } \\
\text { Order }\end{array}$} & \multicolumn{2}{|r|}{$\begin{array}{c}\text { 科 } \\
\text { Family }\end{array}$} & \multicolumn{2}{|r|}{$\begin{array}{l}\text { 物种名 } \\
\text { Species }\end{array}$} & \multirow{2}{*}{$\begin{array}{l}\text { 分布型 } \\
\text { Chorotype } \\
\text { si }\end{array}$} & \multirow[t]{2}{*}{$\begin{array}{c}\text { 备注 } \\
\text { Remarks }\end{array}$} \\
\hline & 鸟纲 & Aves & 鸡形目 & Galliformes & 雉科 & Phasianidae & 红腹锦鸡 & Chrysolophus pictus & & \\
\hline 770 & 鸟纲 & Aves & 鸡形目 & Galliformes & 雉科 & Phasianidae & 白腹锦鸡 & Chrysolophus amherstiae & he & \\
\hline 771 & 鸟纲 & Aves & 鸡形目 & Galliformes & 雉科 & Phasianidae & 灰孔雀雉 & Polyplectron bicalcaratum & wa & \\
\hline 772 & 鸟纲 & Aves & 鸡形目 & Galliformes & 雉科 & Phasianidae & 绿孔雀 & Pavo muticus & wa & \\
\hline 773 & 鸟纲 & Aves & 鹤形目 & Gruiformes & 三趾敦科 & Turnicidae & 林三趾敦 & Turnix sylvaticus & wa & \\
\hline 774 & 鸟纲 & Aves & 鹤形目 & Gruiformes & 三趾敦科 & Turnicidae & 棕三趾敦 & Turnix suscitator & wb & \\
\hline 775 & 鸟纲 & Aves & 鹤形目 & Gruiformes & 鹤科 & Gruidae & 㝨羽鹤 & Grus virgo & d & \\
\hline 776 & 鸟纲 & Aves & 鹤形目 & Gruiformes & 鹤科 & Gruidae & 白枕鹤 & Grus vipio & $\mathrm{ma}$ & \\
\hline 777 & 鸟纲 & Aves & 鹤形目 & Gruiformes & 鹤科 & Gruidae & 灰鹤 & Grus grus & ub & \\
\hline 778 & 鸟纲 & Aves & 鹤形目 & Gruiformes & 鹤科 & Gruidae & 白头鹤 & Grus monacha & $\mathrm{m}$ & \\
\hline 779 & 鸟纲 & Aves & 鹤形目 & Gruiformes & 鹤科 & Gruidae & 黑颈鹤 & Grus nigricollis & $\mathrm{pc}$ & \\
\hline 780 & 鸟纲 & Aves & 鹤形目 & Gruiformes & 鹤科 & Gruidae & 丹顶鹤 & Grus japonensis & ma & \\
\hline 781 & 鸟纲 & Aves & 鹤形目 & Gruiformes & 秧鸡科 & Rallidae & 长脚秧鸡 & Crex crex & o & \\
\hline 782 & 鸟纲 & Aves & 鹤形目 & Gruiformes & 秧鸡科 & Rallidae & 姬田鸡 & Porzana parva & o & \\
\hline 783 & 鸟纲 & Aves & 鹤形目 & Gruiformes & 秩鸡科 & Rallidae & 小田鸡 & Porzana pusilla & o & \\
\hline 784 & 鸟纲 & Aves & 鹤形目 & Gruiformes & 秩鸡科 & Rallidae & 斑胸田鸡 & Porzana porzana & ud & \\
\hline 785 & 鸟纲 & Aves & 鹤形目 & Gruiformes & 秧鸡科 & Rallidae & 棕背田鸡 & Porzana bicolor & wc & \\
\hline 786 & 鸟纲 & Aves & 鹤形目 & Gruiformes & 秩鸡科 & Rallidae & 紫水鸡 & Porphyrio porphyrio & o1 & \\
\hline 787 & 鸟纲 & Aves & 鹤形目 & Gruiformes & 秧鸡科 & Rallidae & 黑水鸡 & Gallinula chloropus & $\mathrm{o} 2$ & \\
\hline 788 & 鸟纲 & Aves & 鹤形目 & Gruiformes & 铇科 & Otididae & 大鸨 & Otis tarda & 03 & \\
\hline 789 & 鸟纲 & Aves & 鹤形目 & Gruiformes & 铇科 & Otididae & 波斑铇 & Chlamydotis macqueenii & 03 & \\
\hline 790 & 鸟纲 & Aves & 鹤形目 & Gruiformes & 铇科 & Otididae & 小铇 & Tetrax tetrax & 03 & \\
\hline
\end{tabular}


郜二虎, 何杰坤, 王志臣, 徐扬, 唐小平, 江海声. 全国陆生野生动物调查单元区划方案. 生物多样性, 2017, 25 (12): 1321-1330.

http://www.biodiversity-science.net/CN/10.17520/biods.2017135

\begin{tabular}{|c|c|c|c|c|c|c|c|c|c|c|}
\hline \multirow{2}{*}{$\begin{array}{l}\text { 序号 } \\
\text { No. } \\
791\end{array}$} & \multicolumn{2}{|c|}{$\begin{array}{c}\text { 纲 } \\
\text { Class }\end{array}$} & \multicolumn{2}{|c|}{$\begin{array}{c}\text { 目 } \\
\text { Order }\end{array}$} & \multirow{2}{*}{\multicolumn{2}{|c|}{$\begin{array}{c}\text { 科 } \\
\text { Family } \\
\text { Jacanidae }\end{array}$}} & \multicolumn{2}{|r|}{$\begin{array}{l}\text { 物种名 } \\
\text { Species }\end{array}$} & \multirow{2}{*}{$\begin{array}{l}\begin{array}{c}\text { 分布型 } \\
\text { Chorotype }\end{array} \\
\text { wa }\end{array}$} & \multirow[t]{2}{*}{$\begin{array}{c}\text { 备注 } \\
\text { Remarks }\end{array}$} \\
\hline & 鸟纲 & Aves & 彻形目 & Charadriiformes & & & 铜翅水雉 & Metopidius indicus & & \\
\hline 792 & 鸟纲 & Aves & 彻形目 & Charadriiformes & 蚛鸼科 & Haematopodidae & 蚛矝 & Haematopus ostralegus & $\mathrm{cf}$ & \\
\hline 793 & 鸟纲 & Aves & 衍形目 & Charadriiformes & 噮嘴鹬科 & Ibidorhynchidae & 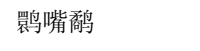 & Ibidorhyncha struthersii & $\mathrm{pf}$ & \\
\hline 794 & 鸟纲 & Aves & 彻形目 & Charadriiformes & 反嘴嘀科 & Recurvirostridae & 黑翅长脚嘀 & Himantopus himantopus & $\mathrm{o} 2$ & \\
\hline 795 & 鸟纲 & Aves & 彻形目 & Charadriiformes & 反嘴璚科 & Recurvirostridae & 反嘴㦷 & Recurvirostra avosetta & 03 & \\
\hline 796 & 鸟纲 & Aves & 衍形目 & Charadriiformes & 衍科 & Charadriidae & 距翅麦鸡 & Vanellus duvaucelii & wa & \\
\hline 797 & 鸟纲 & Aves & 彻形目 & Charadriiformes & 衍科 & Charadriidae & 肉垂麦鸡 & Vanellus indicus & wa & \\
\hline 798 & 鸟纲 & Aves & 彻形目 & Charadriiformes & 衍科 & Charadriidae & 蒙古沙衍 & Charadrius mongolus & d & \\
\hline 799 & 鸟纲 & Aves & 彻形目 & Charadriiformes & 钧科 & Charadriidae & 铁嘴沙鸭 & Charadrius leschenaultii & da & \\
\hline 800 & 鸟纲 & Aves & 彻形目 & Charadriiformes & 衍科 & Charadriidae & 红胸彻 & Charadrius asiaticus & $\mathrm{d}$ & \\
\hline 801 & 鸟纲 & Aves & 衍形目 & Charadriiformes & 商科 & Scolopacidae & 丘鹬 & Scolopax rusticola & ud & \\
\hline 802 & 鸟纲 & Aves & 彻形目 & Charadriiformes & 㦷科 & Scolopacidae & 孤沙雉 & Gallinago solitaria & $\mathrm{u}$ & \\
\hline 803 & 鸟纲 & Aves & 彻形目 & Charadriiformes & 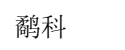 & Scolopacidae & 林沙雉 & Gallinago nemoricola & wa & \\
\hline 804 & 鸟纲 & Aves & 衍形目 & Charadriiformes & 鹝科 & Scolopacidae & 针尾沙雉 & Gallinago stenura & uc & \\
\hline 805 & 鸟纲 & Aves & 衍形目 & Charadriiformes & 㷰科 & Scolopacidae & 扇尾沙雉 & Gallinago gallinago & $\mathrm{ub}$ & \\
\hline 806 & 鸟纲 & Aves & 彻形目 & Charadriiformes & 缡科 & Scolopacidae & 黑尾塍鹋 & Limosa limosa & uc & \\
\hline 807 & 鸟纲 & Aves & 彻形目 & Charadriiformes & 㦷科 & Scolopacidae & 白腰杓鹬 & Numenius arquata & ud & \\
\hline 808 & 鸟纲 & Aves & 衍形目 & Charadriiformes & 㦷科 & Scolopacidae & 大杓憰 & Numenius madagascariensis & $\mathrm{ma}$ & \\
\hline 809 & 鸟纲 & Aves & 衍形目 & Charadriiformes & 裔科 & Scolopacidae & 泽鹬 & Tringa stagnatilis & $\mathrm{u}$ & \\
\hline 810 & 鸟纲 & Aves & 鸿形目 & Charadriiformes & 㦷科 & Scolopacidae & 青脚䂆岛 & Tringa nebularia & uc & \\
\hline 811 & 鸟纲 & Aves & 彻形目 & Charadriiformes & 㦷科 & Scolopacidae & 白腰草䟱 & Tringa ochropus & uc & \\
\hline 812 & 鸟纲 & Aves & 彻形目 & Charadriiformes & 騆科 & Scolopacidae & 林覶 & Tringa glareola & ua & \\
\hline
\end{tabular}


郜二虎, 何杰坤, 王志臣, 徐扬, 唐小平, 江海声. 全国陆生野生动物调查单元区划方案. 生物多样性, 2017, 25 (12): 1321-1330.

http://www.biodiversity-science.net/CN/10.17520/biods.2017135

\begin{tabular}{|c|c|c|c|c|c|c|c|c|c|c|}
\hline \multirow{2}{*}{$\begin{array}{l}\text { 序号 } \\
\text { No. } \\
813\end{array}$} & \multicolumn{2}{|c|}{$\begin{array}{c}\text { 纲 } \\
\text { Class }\end{array}$} & \multicolumn{2}{|c|}{$\begin{array}{c}\text { 目 } \\
\text { Order }\end{array}$} & \multicolumn{2}{|r|}{$\begin{array}{c}\text { 科 } \\
\text { Family }\end{array}$} & \multicolumn{2}{|r|}{$\begin{array}{l}\text { 物种名 } \\
\text { Species }\end{array}$} & \multirow{2}{*}{$\begin{array}{l}\begin{array}{c}\text { 分布型 } \\
\text { Chorotype }\end{array} \\
\text { cf }\end{array}$} & \multirow[t]{2}{*}{$\begin{array}{c}\text { 备注 } \\
\text { Remarks }\end{array}$} \\
\hline & 鸟纲 & Aves & 彻形目 & Charadriiformes & 鸸科 & Scolopacidae & 矶鹝 & Actitis hypoleucos & & \\
\hline 814 & 鸟纲 & Aves & 彻形目 & Charadriiformes & 鸥科 & Laridae & 渔鸥 & Larus ichthyaetus & $\mathrm{d}$ & \\
\hline 815 & 鸟纲 & Aves & 衍形目 & Charadriiformes & 鸥科 & Laridae & 棕头鸥 & Larus brunnicephalus & pa & \\
\hline 816 & 鸟纲 & Aves & 衍形目 & Charadriiformes & 鸥科 & Laridae & 黑嘴鸥 & Larus saundersi & $\mathrm{m}$ & \\
\hline 817 & 鸟纲 & Aves & 衍形目 & Charadriiformes & 鸥科 & Laridae & 遗鸥 & Larus relictus & $\mathrm{d}$ & \\
\hline 818 & 鸟纲 & Aves & 衍形目 & Charadriiformes & 鸥科 & Laridae & 小鸥 & Larus minutus & uc & \\
\hline 819 & 鸟纲 & Aves & 衍形目 & Charadriiformes & 燕鸥科 & Sternidae & 鸥嘴噪鸥 & Gelochelidon nilotica & $\mathrm{o} 2$ & \\
\hline 820 & 鸟纲 & Aves & 衍形目 & Charadriiformes & 燕鸥科 & Sternidae & 大凤头燕鸥 & Thalasseus bergii & o5 & \\
\hline 821 & 鸟纲 & Aves & 彻形目 & Charadriiformes & 燕鸥科 & Sternidae & 河燕鸥 & Sterna aurantia & wa & \\
\hline 822 & 鸟纲 & Aves & 衍形目 & Charadriiformes & 燕鸥科 & Sternidae & 白额燕鸥 & Sterna albifrons & o2 & \\
\hline 823 & 鸟纲 & Aves & 衍形目 & Charadriiformes & 燕鸥科 & Sternidae & 黑腹燕鸥 & Sterna acuticauda & $\mathrm{m}$ & \\
\hline 824 & 鸟纲 & Aves & 微形目 & Charadriiformes & 燕鸥科 & Sternidae & 灰翅燕鸥 & Chlidonias hybrida & uh & \\
\hline 825 & 鸟纲 & Aves & 衍形目 & Charadriiformes & 燕鸥科 & Sternidae & 白翅燕鸥 & Chlidonias leucopterus & uc & \\
\hline 826 & 鸟纲 & Aves & 衍形目 & Charadriiformes & 燕鸥科 & Sternidae & 黑浮鸥 & Chlidonias niger & $\mathrm{c}$ & \\
\hline 827 & 鸟纲 & Aves & 沙鸡目 & Pterocliformes & 沙鸡科 & Pteroclidae & 西藏毛腿沙鸡 & Syrrhaptes tibetanus & pa & \\
\hline 828 & 鸟纲 & Aves & 沙鸡目 & Pterocliformes & 沙鸡科 & Pteroclidae & 毛腿沙鸡 ～～～ & Syrrhaptes paradoxus & da & \\
\hline 829 & 鸟纲 & Aves & 沙鸡目 & Pterocliformes & 沙鸡科 & Pteroclidae & 黑腹沙鸡 & Pterocles orientalis & df & \\
\hline 830 & 鸟纲 & Aves & 鸽形目 & Columbiformes & 鸭鸽科 & Columbidae & 原鸽 & Columba livia & 03 & \\
\hline 831 & 鸟纲 & Aves & 鸽形目 & Columbiformes & 鸭鸽科 & Columbidae & 雪鸽 & Columba leuconota & $\mathrm{hm}$ & \\
\hline 832 & 鸟纲 & Aves & 鸽形目 & Columbiformes & 鸠鸽科 & Columbidae & 欧鸽 & Columba oenas & 03 & \\
\hline 833 & 鸟纲 & Aves & 鸽形目 & Columbiformes & 鸭鸽科 & Columbidae & 中亚鸽 & Columba eversmanni & 03 & \\
\hline 834 & 鸟纲 & Aves & 鸽形目 & Columbiformes & 扸崄科 & Columbidae & 斑尾林鸽 & Columba palumbus & o & \\
\hline
\end{tabular}


郜二虎, 何杰坤, 王志臣, 徐扬, 唐小平, 江海声. 全国陆生野生动物调查单元区划方案. 生物多样性, 2017, 25 (12): 1321-1330.

http://www.biodiversity-science.net/CN/10.17520/biods.2017135

\begin{tabular}{|c|c|c|c|c|c|c|c|c|c|c|}
\hline \multirow{2}{*}{$\begin{array}{l}\text { 序号 } \\
\text { No. } \\
835\end{array}$} & \multicolumn{2}{|c|}{$\begin{array}{c}\text { 纲 } \\
\text { Class }\end{array}$} & \multicolumn{2}{|c|}{$\begin{array}{c}\text { 目 } \\
\text { Order }\end{array}$} & \multicolumn{2}{|r|}{$\begin{array}{c}\text { 科 } \\
\text { Family }\end{array}$} & \multicolumn{2}{|r|}{$\begin{array}{l}\text { 物种名 } \\
\text { Species }\end{array}$} & \multirow{2}{*}{$\begin{array}{l}\text { 分布型 } \\
\text { Chorotype }\end{array}$} & \multirow[t]{2}{*}{$\begin{array}{c}\text { 备注 } \\
\text { Remarks }\end{array}$} \\
\hline & 鸟纲 & Aves & 鸽形目 & Columbiformes & 鸭鸽科 & Columbidae & 灰林鸽 & Columba pulchricollis & & \\
\hline 836 & 鸟纲 & Aves & 鸽形目 & Columbiformes & 鸭鸽科 & Columbidae & 紫林鸽 & Columba punicea & wa & \\
\hline 837 & 鸟纲 & Aves & 鸽形目 & Columbiformes & 鹏鸽科 & Columbidae & 欧斑鸭 & Streptopelia turtur & o & \\
\hline 838 & 鸟纲 & Aves & 鸽形目 & Columbiformes & 鸭鸽科 & Columbidae & 山斑鸠 & Streptopelia orientalis & e & \\
\hline 839 & 鸟纲 & Aves & 鸽形目 & Columbiformes & 鸭鸽科 & Columbidae & 灰斑鸭 & Streptopelia decaocto & we & \\
\hline 840 & 鸟纲 & Aves & 鸽形目 & Columbiformes & 鸭鸽科 & Columbidae & 棕斑鸭 & Streptopelia senegalensis & o & \\
\hline 841 & 鸟纲 & Aves & 鸽形目 & Columbiformes & 鸭鸽科 & Columbidae & 斑尾鹃加 & Macropygia unchall & wd & \\
\hline 842 & 鸟纲 & Aves & 鸽形目 & Columbiformes & 扸鸽科 & Columbidae & 小鹃鸤 & Macropygia ruficeps & wa & \\
\hline 843 & 鸟纲 & Aves & 鸽形目 & Columbiformes & 鸭鸽科 & Columbidae & 绿翅金鸤 & Chalcophaps indica & wb & \\
\hline 844 & 鸟纲 & Aves & 鸽形目 & Columbiformes & 鸭鸽科 & Columbidae & 灰头绿加 & Treron pompadora & wa & \\
\hline 845 & 鸟纲 & Aves & 鸽形目 & Columbiformes & 鹏鸽科 & Columbidae & 针尾绿加 & Treron apicauda & wb & \\
\hline 846 & 鸟纲 & Aves & 鸽形目 & Columbiformes & 鸭鸽科 & Columbidae & 楔尾绿鸠 & Treron sphenurus & wb & \\
\hline 847 & 鸟纲 & Aves & 鸽形目 & Columbiformes & 鸠鸽科 & Columbidae & 绿皇鸭 & Ducula aenea & wa & \\
\hline 848 & 鸟纲 & Aves & 鹦形目 & Psittaciformes & 鹦武科 & Psittacidae & 短尾鹦鹉 & Loriculus vernalis & wa & \\
\hline 849 & 鸟纲 & Aves & 鹦形目 & Psittaciformes & 鹦牪科 & Psittacidae & 红领绿鹦鹉 & Psittacula krameri & wa & \\
\hline 850 & 鸟纲 & Aves & 鹦形目 & Psittaciformes & 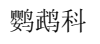 & Psittacidae & 灰头鹦鹉 & Psittacula finschii & $\mathrm{hm}$ & \\
\hline 851 & 鸟纲 & Aves & 鹦形目 & Psittaciformes & 鹦鹉科 & Psittacidae & 花头鹦鹉 & Psittacula roseata & wa & \\
\hline 852 & 鸟纲 & Aves & 鹦形目 & Psittaciformes & 鹦鹉科 & Psittacidae & 大紫胸繁武 & Psittacula derbiana & he & \\
\hline 853 & 鸟纲 & Aves & 鸭形目 & Psittaciformes & 鹦武科 & Psittacidae & 绯胸鹦鹉 & Psittacula alexandri & wa & \\
\hline 854 & 鸟纲 & Aves & 鹃形目 & Cuculiformes & 杜鹃科 & Cuculidae & 栗斑杜鹃 & Cuculus sonneratii & wc & \\
\hline 855 & 鸟纲 & Aves & 鹃形目 & Cuculiformes & 杜鹃科 & Cuculidae & 紫金鹃 & Chalcites xanthorhynchus & wa & \\
\hline 856 & 鸟纲 & Aves & 鹃形目 & Cuculiformes & 杜鹃科 & Cuculidae & 褐翅鸦鹃 & Centropus sinensis & $\mathrm{wb}$ & \\
\hline
\end{tabular}


郜二虎, 何杰坤, 王志臣, 徐扬, 唐小平, 江海声. 全国陆生野生动物调查单元区划方案. 生物多样性, 2017, 25 (12): 1321-1330.

http://www.biodiversity-science.net/CN/10.17520/biods.2017135

\begin{tabular}{|c|c|c|c|c|c|c|c|c|c|c|}
\hline $\begin{array}{r}\text { 序号 } \\
\text { No. }\end{array}$ & \multicolumn{2}{|c|}{$\begin{array}{c}\text { 纲 } \\
\text { Class }\end{array}$} & \multicolumn{2}{|c|}{$\begin{array}{c}\text { 目 } \\
\text { Order }\end{array}$} & \multicolumn{2}{|r|}{$\begin{array}{c}\text { 科 } \\
\text { Family }\end{array}$} & \multicolumn{2}{|r|}{$\begin{array}{l}\text { 物种名 } \\
\text { Species }\end{array}$} & \multirow{2}{*}{$\begin{array}{l}\begin{array}{c}\text { 分布型 } \\
\text { Chorotype }\end{array} \\
\text { o1 }\end{array}$} & \multirow[t]{2}{*}{$\begin{array}{c}\text { 备注 } \\
\text { Remarks }\end{array}$} \\
\hline 857 & 鸟纲 & Aves & 鸮形目 & Strigiformes & 草鸮科 & Tytonidae & 东方草鸮 & Tyto longimembris & & \\
\hline 858 & 鸟纲 & Aves & 鸮形目 & Strigiformes & 草鸮科 & Tytonidae & 栗鸮 & Phodilus badius & wa & \\
\hline 859 & 鸟纲 & Aves & 鸮形目 & Strigiformes & 鸱鸮科 & Strigidae & 黄嘴角鸮 & Otus spilocephalus & $\mathrm{wb}$ & \\
\hline 860 & 鸟纲 & Aves & 鸮形目 & Strigiformes & 鸱鸮科 & Strigidae & 领角鸮 & Otus lettia & we & \\
\hline 861 & 鸟纲 & Aves & 鸮形目 & Strigiformes & 鸱鸮科 & Strigidae & 雕鸮 & Bubo bubo & uh & \\
\hline 862 & 鸟纲 & Aves & 鸮形目 & Strigiformes & 鸱鸮科 & Strigidae & 林雕鸮 & Bubo nipalensis & wc & \\
\hline 863 & 鸟纲 & Aves & 鸮形目 & Strigiformes & 鸱鸮科 & Strigidae & 毛腿渔鸮 & Ketupa blakistoni & $\mathrm{m}$ & \\
\hline 864 & 鸟纲 & Aves & 鸮形目 & Strigiformes & 鸱鸮科 & Strigidae & 褐渔鸮 & Ketupa zeylonensis & $\mathrm{wb}$ & \\
\hline 865 & 鸟纲 & Aves & 鸮形目 & Strigiformes & 鸱鸮科 & Strigidae & 黄腿渔鸮 & Ketupa flavipes & wd & \\
\hline 866 & 鸟纲 & Aves & 鸮形目 & Strigiformes & 鸱鸮科 & Strigidae & 褐林鸮 & Strix leptogrammica & wc & \\
\hline 867 & 鸟纲 & Aves & 鸮形目 & Strigiformes & 鸱鸮科 & Strigidae & 灰林鸮 & Strix aluco & o1 & \\
\hline 868 & 鸟纲 & Aves & 鸮形目 & Strigiformes & 鸱鸮科 & Strigidae & 长尾林鸮 & Strix uralensis & uc & \\
\hline 869 & 鸟纲 & Aves & 鸮形目 & Strigiformes & 鸱鸮科 & Strigidae & 乌林鸮 & Strix nebulosa & $\mathrm{cc}$ & \\
\hline 870 & 鸟纲 & Aves & 鸮形目 & Strigiformes & 鸱鸮科 & Strigidae & 领鸺鹠 & Glaucidium brodiei & we & \\
\hline 871 & 鸟纲 & Aves & 鸮形目 & Strigiformes & 鸱鸮科 & Strigidae & 斑头鸺鹠 & Glaucidium cuculoides & wd & \\
\hline 872 & 鸟纲 & Aves & 鸮形目 & Strigiformes & 鸱鸮科 & Strigidae & 纵纹腹小鸮 & Athene noctua & uf & \\
\hline 873 & 鸟纲 & Aves & 鸮形目 & Strigiformes & 鸱鸮科 & Strigidae & 鬼鸮 & Aegolius funereus & $\mathrm{cc}$ & \\
\hline 874 & 鸟纲 & Aves & 鸮形目 & Strigiformes & 鸱鸮科 & Strigidae & 长耳鸮 & Asio otus & $\mathrm{cd}$ & \\
\hline 875 & 鸟纲 & Aves & 夜鹰目 & Caprimulgiformes & 蛙口夜鹰科 & Podargidae & 黑顶蛙口夜鹰 & Batrachostomus hodgsoni & wa & \\
\hline 876 & 鸟纲 & Aves & 夜鹰目 & Caprimulgiformes & 夜鹰科 & Caprimulgidae & 欧夜鹰 & Caprimulgus europaeus & 03 & \\
\hline 877 & 鸟纲 & Aves & 夜鹰目 & Caprimulgiformes & 夜鹰科 & Caprimulgidae & 埃及夜鹰 & Caprimulgus aegyptius & o & \\
\hline 878 & 鸟纲 & Aves & 夜鹰目 & Caprimulgiformes & 夜鹰科 & Caprimulgidae & 林夜鹰 & Caprimulgus affinis & wb & \\
\hline
\end{tabular}


郜二虎, 何杰坤, 王志臣, 徐扬, 唐小平, 江海声. 全国陆生野生动物调查单元区划方案. 生物多样性, 2017, 25 (12): 1321-1330.

http://www.biodiversity-science.net/CN/10.17520/biods.2017135

\begin{tabular}{|c|c|c|c|c|c|c|c|c|c|c|}
\hline $\begin{array}{r}\text { 序号 } \\
\text { No. }\end{array}$ & \multicolumn{2}{|c|}{$\begin{array}{c}\text { 纲 } \\
\text { Class }\end{array}$} & \multicolumn{2}{|c|}{$\begin{array}{c}\text { 目 } \\
\text { Order }\end{array}$} & \multicolumn{2}{|r|}{$\begin{array}{c}\text { 科 } \\
\text { Family }\end{array}$} & \multicolumn{2}{|r|}{$\begin{array}{l}\text { 物种名 } \\
\text { Species }\end{array}$} & \multirow{2}{*}{$\begin{array}{l}\begin{array}{c}\text { 分布型 } \\
\text { Chorotype }\end{array} \\
\text { o1 }\end{array}$} & \multirow[t]{2}{*}{$\begin{array}{c}\text { 备注 } \\
\text { Remarks }\end{array}$} \\
\hline 879 & 鸟纲 & Aves & 雨燕目 & Apodiformes & 雨燕科 & Apodidiae & 普通雨燕 & Apus apus & & \\
\hline 880 & 鸟纲 & Aves & 雨燕目 & Apodiformes & 雨燕科 & Apodidiae & 白腰雨燕 & Apus pacificus & $\mathrm{m}$ & \\
\hline 881 & 鸟纲 & Aves & 雨燕目 & Apodiformes & 雨燕科 & Apodidiae & 小白腰雨燕 & Apus nipalensis & $\mathrm{o} 1$ & \\
\hline 882 & 鸟纲 & Aves & 雨燕目 & Apodiformes & 凤头雨燕科 & Hemiprocnidae & 凤头雨燕 & Hemiprocne coronata & wa & \\
\hline 883 & 鸟纲 & Aves & 咬鹃目 & Trogoniformes & 咬鹃科 & Trogonidae & 红头咬鹃 & Harpactes erythrocephalus & wc & \\
\hline 884 & 鸟纲 & Aves & 咬鹃目 & Trogoniformes & 咬鹃科 & Trogonidae & 橙胸咬鹃 & Harpactes oreskios & wa & \\
\hline 885 & 鸟纲 & Aves & 咬鹃目 & Trogoniformes & 咬鹃科 & Trogonidae & 红腹咬鹃 & Harpactes wardi & wa & \\
\hline 886 & 鸟纲 & Aves & 佛法僧目 & Coraciiformes & 翠鸟科 & Alcedinidae & 普通翠鸟 & Alcedo atthis & o1 & \\
\hline 887 & 鸟纲 & Aves & 佛法僧目 & Coraciiformes & 翠鸟科 & Alcedinidae & 蓝耳翠鸟 & Alcedo meninting & wa & \\
\hline 888 & 鸟纲 & Aves & 佛法僧目 & Coraciiformes & 翠鸟科 & Alcedinidae & 三趾翠鸟 & Ceyx erithaca & wa & \\
\hline 889 & 鸟纲 & Aves & 佛法僧目 & Coraciiformes & 翠鸟科 & Alcedinidae & 鹳嘴翡翠 & Pelargopsis capensis & wa & \\
\hline 890 & 鸟纲 & Aves & 佛法僧目 & Coraciiformes & 翠鸟科 & Alcedinidae & 白胸翡翠 & Halcyon smyrnensis & o1 & \\
\hline 891 & 鸟纲 & Aves & 佛法僧目 & Coraciiformes & 翠鸟科 & Alcedinidae & 冠鱼狗 & Megaceryle lugubris & o1 & \\
\hline 892 & 鸟纲 & Aves & 佛法僧目 & Coraciiformes & 翠鸟科 & Alcedinidae & 斑鱼狗 & Ceryle rudis & o1 & \\
\hline 893 & 鸟纲 & Aves & 佛法僧目 & Coraciiformes & 蜂虎科 & Meropidae & 黄喉蜂虎 & Merops apiaster & o & \\
\hline 894 & 鸟纲 & Aves & 佛法僧目 & Coraciiformes & 佛法僧科 & Coraciidae & 蓝胸佛法僧 & Coracias garrulus & o & \\
\hline 895 & 鸟纲 & Aves & 犀鸟目 & Bucerotiformes & 犀鸟科 & Bucerotidae & 冠斑犀鸟 & Anthracoceros albirostris & wa & \\
\hline 896 & 鸟纲 & Aves & 犀鸟目 & Bucerotiformes & 犀鸟科 & Bucerotidae & 双角犀鸟 & Buceros bicornis & wa & \\
\hline 897 & 鸟纲 & Aves & 犀鸟目 & Bucerotiformes & 犀鸟科 & Bucerotidae & 白喉犀鸟 & Anorrhinus tickelli & wa & \\
\hline 898 & 鸟纲 & Aves & 犀鸟目 & Bucerotiformes & 犀鸟科 & Bucerotidae & 棕颈犀鸟 & Aceros nipalensis & wa & \\
\hline 899 & 鸟纲 & Aves & 犀鸟目 & Bucerotiformes & 犀鸟科 & Bucerotidae & 花冠皱盔犀鸟 & Aceros undulatus & wa & \\
\hline 900 & 鸟纲 & Aves & 鴷形目 & Piciformes & 拟殠科 & Capitonidae & 大拟豚木鸟 & Megalaima virens & wc & \\
\hline
\end{tabular}


郜二虎, 何杰坤, 王志臣, 徐扬, 唐小平, 江海声. 全国陆生野生动物调查单元区划方案. 生物多样性, 2017, 25 (12): 1321-1330.

http://www.biodiversity-science.net/CN/10.17520/biods.2017135

\begin{tabular}{|c|c|c|c|c|c|c|c|c|c|c|}
\hline \multirow{2}{*}{$\begin{array}{l}\text { 序号 } \\
\text { No. } \\
901\end{array}$} & \multicolumn{2}{|c|}{$\begin{array}{c}\text { 纲 } \\
\text { Class }\end{array}$} & \multicolumn{2}{|c|}{$\begin{array}{c}\text { 目 } \\
\text { Order }\end{array}$} & \multicolumn{2}{|r|}{$\begin{array}{c}\text { 科 } \\
\text { Family }\end{array}$} & \multicolumn{2}{|r|}{$\begin{array}{l}\text { 物种名 } \\
\text { Species }\end{array}$} & \multirow{2}{*}{$\begin{array}{l}\text { 分布型 } \\
\text { Chorotype }\end{array}$} & \multirow[t]{2}{*}{$\begin{array}{c}\text { 备注 } \\
\text { Remarks }\end{array}$} \\
\hline & 鸟纲 & Aves & 列形目 & Piciformes & 拟列科 & Capitonidae & 黄纹拟豚木鸟 & Megalaima faiostricta & & \\
\hline 902 & 鸟纲 & Aves & 烮形目 & Piciformes & 拟列科 & Capitonidae & 金喉拟豚木鸟 & Megalaima franklinii & wa & \\
\hline 903 & 鸟纲 & Aves & 列形目 & Piciformes & 拟列科 & Capitonidae & 黑眉拟豚木鸟 & Megalaima oorti & wa & \\
\hline 904 & 鸟纲 & Aves & 列形目 & Piciformes & 拟列科 & Capitonidae & 蓝喉拟豚木鸟 & Megalaima asiatica & wb & \\
\hline 905 & 鸟纲 & Aves & 列形目 & Piciformes & 拟列科 & Capitonidae & 蓝耳拟豚木鸟 & Megalaima australis & wa & \\
\hline 906 & 鸟纲 & Aves & 烮形目 & Piciformes & 拟列科 & Capitonidae & 赤胸拟豚木鸟 & Megalaima haemacephala & wa & \\
\hline 907 & 鸟纲 & Aves & 列形目 & Piciformes & 响蜜殈科 & Indicatoridae & 黄腰响蜜㡂 & Indicator xanthonotus & wc & \\
\hline 908 & 鸟纲 & Aves & 耗形目 & Piciformes & 啄木鸟科 & Picidae & 斑姬啄木鸟 & Picumnus innominatus & wd & \\
\hline 909 & 鸟纲 & Aves & 列形目 & Piciformes & 豚木鸟科 & Picidae & 白眉棕豚木鸟 & Sasia ochracea & wa & \\
\hline 910 & 鸟纲 & Aves & 耗形目 & Piciformes & 啄木鸟科 & Picidae & 星头啄木鸟 & Dendrocopos canicapillus & we & \\
\hline 911 & 鸟纲 & Aves & 列形目 & Piciformes & 啄木鸟科 & Picidae & 小星头豚木鸟 & Dendrocopos kizuki & $\mathrm{mb}$ & \\
\hline 912 & 鸟纲 & Aves & 䴕形目 & Piciformes & 豚木鸟科 & Picidae & 小斑豚木鸟 & Dendrocopos minor & uc & \\
\hline 913 & 鸟纲 & Aves & 列形目 & Piciformes & 豚木鸟科 & Picidae & 纹胸啄木鸟 & Dendrocopos atratus & wa & \\
\hline 914 & 鸟纲 & Aves & 㡂形目 & Piciformes & 啄木鸟科 & Picidae & 黄颈啄木鸟 & Dendrocopos darjellensis & $\mathrm{hm}$ & \\
\hline 915 & 鸟纲 & Aves & 列形目 & Piciformes & 豚木鸟科 & Picidae & 赤胸啄木鸟 & Dendrocopos cathpharius & $\mathrm{hm}$ & \\
\hline 916 & 鸟纲 & Aves & 列形目 & Piciformes & 豚木鸟科 & Picidae & 白背啄木鸟 & Dendrocopos leucotos & ug & \\
\hline 917 & 鸟纲 & Aves & 鴷形目 & Piciformes & 豚木鸟科 & Picidae & 大斑豚木鸟 & Dendrocopos major & uc & \\
\hline 918 & 鸟纲 & Aves & 耗形目 & Piciformes & 豚木鸟科 & Picidae & 白翅豚木鸟 & Dendrocopos leucopterus & da & \\
\hline 919 & 鸟纲 & Aves & 暍形目 & Piciformes & 豚木鸟科 & Picidae & 三趾豚木鸟 & Picoides tridactylus & $\mathrm{cc}$ & \\
\hline 920 & 鸟纲 & Aves & 㡂形目 & Piciformes & 豚木鸟科 & Picidae & 栗豚木鸟 & Celeus brachyurus & $\mathrm{wb}$ & \\
\hline 921 & 鸟纲 & Aves & 烮形目 & Piciformes & 豚木鸟科 & Picidae & 白腹黑豚木鸟 & Dryocopus javensis & wc & \\
\hline 922 & 鸟纲 & Aves & 裂形目 & Piciformes & 啄木鸟科 & Picidae & 黑豚木鸟 & Dryocopus martius & uc & \\
\hline
\end{tabular}


郜二虎, 何杰坤, 王志臣, 徐扬, 唐小平, 江海声. 全国陆生野生动物调查单元区划方案. 生物多样性, 2017, 25 (12): 1321-1330.

http://www.biodiversity-science.net/CN/10.17520/biods.2017135

\begin{tabular}{|c|c|c|c|c|c|c|c|c|c|c|}
\hline \multirow{2}{*}{$\begin{array}{l}\text { 序号 } \\
\text { No. } \\
923\end{array}$} & \multicolumn{2}{|c|}{$\begin{array}{c}\text { 纲 } \\
\text { Class }\end{array}$} & \multicolumn{2}{|c|}{$\begin{array}{c}\text { 目 } \\
\text { Order }\end{array}$} & \multicolumn{2}{|r|}{$\begin{array}{c}\text { 科 } \\
\text { Family }\end{array}$} & \multicolumn{2}{|r|}{$\begin{array}{l}\text { 物种名 } \\
\text { Species }\end{array}$} & \multirow{2}{*}{$\begin{array}{l}\begin{array}{c}\text { 分布型 } \\
\text { Chorotype }\end{array} \\
\text { wb }\end{array}$} & \multirow[t]{2}{*}{$\begin{array}{c}\text { 备注 } \\
\text { Remarks }\end{array}$} \\
\hline & 鸟纲 & Aves & 列形目 & Piciformes & 豚木鸟科 & Picidae & 黄冠豚木鸟 & Picus chlorolophus & & \\
\hline 924 & 鸟纲 & Aves & 烮形目 & Piciformes & 啄木鸟科 & Picidae & 大黄冠啄木鸟 & Picus flavinucha & wc & \\
\hline 925 & 鸟纲 & Aves & 裂形目 & Piciformes & 啄木鸟科 & Picidae & 花腹绿啄木鸟 & Picus vittatus & wa & \\
\hline 926 & 鸟纲 & Aves & 列形目 & Piciformes & 豚木鸟科 & Picidae & 鳞喉绿豚木鸟 & Picus xanthopygaeus & wa & \\
\hline 927 & 鸟纲 & Aves & 列形目 & Piciformes & 豚木鸟科 & Picidae & 鳞腹绿豚木鸟 & Picus squamatus & o & \\
\hline 928 & 鸟纲 & Aves & 烮形目 & Piciformes & 豚木鸟科 & Picidae & 红颈绿豚木鸟 & Picus rabieri & wa & \\
\hline 929 & 鸟纲 & Aves & 暍形目 & Piciformes & 豚木鸟科 & Picidae & 灰头绿豚木鸟 & Picus canus & uh & \\
\hline 930 & 鸟纲 & Aves & 列形目 & Piciformes & 豚木鸟科 & Picidae & 金背啄木鸟 & Dinopium javanense & wa & \\
\hline 931 & 鸟纲 & Aves & 列形目 & Piciformes & 豚木鸟科 & Picidae & 竹啄木鸟 & Gecinulus grantia & wb & \\
\hline 932 & 鸟纲 & Aves & 列形目 & Piciformes & 豚木鸟科 & Picidae & 黄嘴栗啄木鸟 & Blythipicus pyrrhotis & wd & \\
\hline 933 & 鸟纲 & Aves & 列形目 & Piciformes & 豚木鸟科 & Picidae & 大灰啄木鸟 & Mulleripicus pulverulentus & wa & \\
\hline 934 & 鸟纲 & Aves & 雀形目 & Passeriformes & 阔嘴鸟科 & Eurylaimidae & 长尾阔嘴鸟 & Psarisomus dalhousiae & wc & \\
\hline 935 & 鸟纲 & Aves & 雀形目 & Passeriformes & 阔嘴鸟科 & Eurylaimidae & 银胸丝冠鸟 & Serilophus lunatus & wa & \\
\hline 936 & 鸟纲 & Aves & 雀形目 & Passeriformes & 八色冻科 & Pittidae & 双㵷八色鸫 & Pitta phayrei & wa & \\
\hline 937 & 鸟纲 & Aves & 雀形目 & Passeriformes & 八色冻科 & Pittidae & 蓝枕八色冻 & Pitta nipalensis & wa & \\
\hline 938 & 鸟纲 & Aves & 雀形目 & Passeriformes & 八色冻科 & Pittidae & 蓝背八色鸫 & Pitta soror & wb & \\
\hline 939 & 鸟纲 & Aves & 雀形目 & Passeriformes & 八色冻科 & Pittidae & 栗头八色鸫 & Pitta oatesi & wa & \\
\hline 940 & 鸟纲 & Aves & 雀形目 & Passeriformes & 八色冻科 & Pittidae & 蓝八色冻 & Pitta cyanea & wa & \\
\hline 941 & 鸟纲 & Aves & 雀形目 & Passeriformes & 八色冻科 & Pittidae & 绿胸八色冻 & Pitta sordida & wa & \\
\hline 942 & 鸟纲 & Aves & 雀形目 & Passeriformes & 百灵科 & Alaudidae & 歌百灵 & Mirafra javanica & o1 & \\
\hline 943 & 鸟纲 & Aves & 雀形目 & Passeriformes & 百灵科 & Alaudidae & 长嘴百灵 & Melanocorypha maxima & pa & \\
\hline 944 & 鸟纲 & Aves & 雀形目 & Passeriformes & 百灵科 & Alaudidae & 蒙古百灵 & Melanocorypha mongolica & $\mathrm{dn}$ & \\
\hline
\end{tabular}


郜二虎, 何杰坤, 王志臣, 徐扬, 唐小平, 江海声. 全国陆生野生动物调查单元区划方案. 生物多样性, 2017, 25 (12): 1321-1330.

http://www.biodiversity-science.net/CN/10.17520/biods.2017135

\begin{tabular}{|c|c|c|c|c|c|c|c|c|c|c|}
\hline \multirow{2}{*}{$\begin{array}{l}\text { 序号 } \\
\text { No. } \\
945\end{array}$} & \multicolumn{2}{|c|}{$\begin{array}{c}\text { 纲 } \\
\text { Class }\end{array}$} & \multicolumn{2}{|c|}{$\begin{array}{c}\text { 目 } \\
\text { Order }\end{array}$} & \multirow{2}{*}{\multicolumn{2}{|c|}{$\begin{array}{c}\text { 科 } \\
\text { Family } \\
\text { Alaudidae }\end{array}$}} & \multicolumn{2}{|r|}{$\begin{array}{l}\text { 物种名 } \\
\text { Species }\end{array}$} & \multirow{2}{*}{$\begin{array}{l}\begin{array}{c}\text { 分布型 } \\
\text { Chorotype }\end{array} \\
\text { ue }\end{array}$} & \multirow[t]{2}{*}{$\begin{array}{c}\text { 备注 } \\
\text { Remarks }\end{array}$} \\
\hline & 鸟纲 & Aves & 雀形目 & Passeriformes & & & 云雀 & Alauda arvensis & & \\
\hline 946 & 鸟纲 & Aves & 雀形目 & Passeriformes & 百灵科 & Alaudidae & 小云雀 & Alauda gulgula & we & \\
\hline 947 & 鸟纲 & Aves & 雀形目 & Passeriformes & 燕科 & Hirundinidae & 崖沙燕 & Riparia riparia & $\mathrm{cg}$ & \\
\hline 948 & 鸟纲 & Aves & 雀形目 & Passeriformes & 燕科 & Hirundinidae & 岩燕 & Ptyonoprogne rupestris & 03 & \\
\hline 949 & 鸟纲 & Aves & 雀形目 & Passeriformes & 燕科 & Hirundinidae & 纯色岩燕 & Ptyonoprogne concolor & wa & \\
\hline 950 & 鸟纲 & Aves & 雀形目 & Passeriformes & 燕科 & Hirundinidae & 家燕 & Hirundo rustica & $\mathrm{ch}$ & \\
\hline 951 & 鸟纲 & Aves & 雀形目 & Passeriformes & 燕科 & Hirundinidae & 金腰燕 & Cecropis dautica & $\mathrm{u}$ & \\
\hline 952 & 鸟纲 & Aves & 雀形目 & Passeriformes & 燕科 & Hirundinidae & 斑腰燕 & Cecropis rustica & wa & \\
\hline 953 & 鸟纲 & Aves & 雀形目 & Passeriformes & 仯鸰科 & Motacillidae & 田鹨 & Anthus richardi & $\mathrm{mf}$ & \\
\hline 954 & 鸟纲 & Aves & 雀形目 & Passeriformes & 能鸰科 & Motacillidae & 平原鹨 & Anthus campestris & $\mathrm{d}$ & \\
\hline 955 & 鸟纲 & Aves & 雀形目 & Passeriformes & 能鸰科 & Motacillidae & 林翏乌 & Anthus trivialis & $\mathrm{u}$ & \\
\hline 956 & 鸟纲 & Aves & 雀形目 & Passeriformes & 仯乌鸰科 & Motacillidae & 树鹋 & Anthus hodgsoni & $\mathrm{m}$ & \\
\hline 957 & 鸟纲 & Aves & 雀形目 & Passeriformes & 绡鸰科 & Motacillidae & 粉红胸翏乌 & Anthus roseatus & pa & \\
\hline 958 & 鸟纲 & Aves & 雀形目 & Passeriformes & 绡鸰科 & Motacillidae & 水翏鸟 & Anthus spinoletta & $\mathrm{c}$ & \\
\hline 959 & 鸟纲 & Aves & 雀形目 & Passeriformes & 能鸰科 & Motacillidae & 山鹨 & Anthus sylvanus & $\mathrm{sc}$ & \\
\hline 960 & 鸟纲 & Aves & 雀形目 & Passeriformes & 山椒鸟科 & Campephagidae & 大鹃鵙 & Coracina macei & wb & \\
\hline 961 & 鸟纲 & Aves & 雀形目 & Passeriformes & 山椒鸟科 & Campephagidae & 粉红山椒鸟 & Pericrocotus roseus & wc & \\
\hline 962 & 鸟纲 & Aves & 雀形目 & Passeriformes & 山椒鸟科 & Campephagidae & 长尾山椒鸟 & Pericrocotus ethologus & $\mathrm{hm}$ & \\
\hline 963 & 鸟纲 & Aves & 雀形目 & Passeriformes & 山椒鸟科 & Campephagidae & 赤红山椒鸟 & Pericrocotus flammeus & wc & \\
\hline 964 & 鸟纲 & Aves & 雀形目 & Passeriformes & 山椒鸟科 & Campephagidae & 灰喉山椒鸟 & Pericrocotus solaris & wc & \\
\hline 965 & 鸟纲 & Aves & 雀形目 & Passeriformes & 山椒鸟科 & Campephagidae & 褐背鉣鵙 & Hemipus picatus & wc & \\
\hline 966 & 鸟纲 & Aves & 雀形目 & Passeriformes & 鹐科 & Pycnonotidae & 领雀嘴塊 & Spizixos semitorques & wc & \\
\hline
\end{tabular}


郜二虎, 何杰坤, 王志臣, 徐扬, 唐小平, 江海声. 全国陆生野生动物调查单元区划方案. 生物多样性, 2017, 25 (12): 1321-1330.

http://www.biodiversity-science.net/CN/10.17520/biods.2017135

\begin{tabular}{|c|c|c|c|c|c|c|c|c|c|c|}
\hline \multirow{2}{*}{$\begin{array}{l}\text { 序号 } \\
\text { No. } \\
967\end{array}$} & \multicolumn{2}{|c|}{$\begin{array}{c}\text { 纲 } \\
\text { Class }\end{array}$} & \multicolumn{2}{|c|}{$\begin{array}{c}\text { 目 } \\
\text { Order }\end{array}$} & \multicolumn{2}{|r|}{$\begin{array}{c}\text { 科 } \\
\text { Family }\end{array}$} & \multicolumn{2}{|r|}{$\begin{array}{l}\text { 物种名 } \\
\text { Species }\end{array}$} & \multirow{2}{*}{$\begin{array}{l}\text { 分布型 } \\
\text { Chorotype }\end{array}$} & \multirow[t]{2}{*}{$\begin{array}{c}\text { 备注 } \\
\text { Remarks }\end{array}$} \\
\hline & 鸟纲 & Aves & 雀形目 & Passeriformes & 鶤科 & Pycnonotidae & 纵纹绿軼 & Pycnonotus striatus & & \\
\hline 968 & 鸟纲 & Aves & 雀形目 & Passeriformes & 鹎科 & Pycnonotidae & 黑头皟 & Pycnonotus atriceps & wa & \\
\hline 969 & 鸟纲 & Aves & 雀形目 & Passeriformes & 舺科 & Pycnonotidae & 黑冠黄鹉 & Pycnonotus melanicterus & wa & \\
\hline 970 & 鸟纲 & Aves & 雀形目 & Passeriformes & 鹋科 & Pycnonotidae & 台湾軥 & Pycnonotus taivanus & $\mathrm{j}$ & \\
\hline 971 & 鸟纲 & Aves & 雀形目 & Passeriformes & 鶤科 & Pycnonotidae & 红耳軼 & Pycnonotus jocosus & wc & \\
\hline 972 & 鸟纲 & Aves & 雀形目 & Passeriformes & 鸼科 & Pycnonotidae & 黄臀䇛 & Pycnonotus xanthorrhous & we & \\
\hline 973 & 鸟纲 & Aves & 雀形目 & Passeriformes & 積科 & Pycnonotidae & 白头鸼 & Pycnonotus sinensis & sd & \\
\hline 974 & 鸟纲 & Aves & 雀形目 & Passeriformes & 積科 & Pycnonotidae & 黑喉红慰軥 & Pycnonotus cafer & wa & \\
\hline 975 & 鸟纲 & Aves & 雀形目 & Passeriformes & 殅科 & Pycnonotidae & 白喉红臀鸼 & Pycnonotus aurigaster & $\mathrm{wb}$ & \\
\hline 976 & 鸟纲 & Aves & 雀形目 & Passeriformes & 鹎科 & Pycnonotidae & 纹喉鹎 & Pycnonotus finlaysoni & wa & \\
\hline 977 & 鸟纲 & Aves & 雀形目 & Passeriformes & 鸼科 & Pycnonotidae & 黄绿䇛 & Pycnonotus flavescens & wa & \\
\hline 978 & 鸟纲 & Aves & 雀形目 & Passeriformes & 鶤科 & Pycnonotidae & 黄腹冠軼 & Alophpixus flaveolus & wa & \\
\hline 979 & 鸟纲 & Aves & 雀形目 & Passeriformes & 鶤科 & Pycnonotidae & 白喉冠軼 & Alophoixus pallidus & wc & \\
\hline 980 & 鸟纲 & Aves & 雀形目 & Passeriformes & 鹎科 & Pycnonotidae & 灰眼短脚鹎 & Iole propinqua & wa & \\
\hline 981 & 鸟纲 & Aves & 雀形目 & Passeriformes & 鸼科 & Pycnonotidae & 栗背短脚鹎 & Hemixos castanonotus & wb & \\
\hline 982 & 鸟纲 & Aves & 雀形目 & Passeriformes & 積科 & Pycnonotidae & 绿翅短脚鹎 & Hypsipetes mcclellandii & wc & \\
\hline 983 & 鸟纲 & Aves & 雀形目 & Passeriformes & 雀鸼科 & Aegithinidae & 黑翅雀鹎 & Aegithina tiphia & wa & \\
\hline 984 & 鸟纲 & Aves & 雀形目 & Passeriformes & 雀鸼科 & Aegithinidae & 大绿雀鸼 & Aegithina lafresnayei & wa & \\
\hline 985 & 鸟纲 & Aves & 雀形目 & Passeriformes & 叶軠科 & Chloropseidae & 橙腹叶積 & Chloropsis hardwickii & wc & \\
\hline 986 & 鸟纲 & Aves & 雀形目 & Passeriformes & 和平鸟科 & Irenidae & 和平鸟 & Irena puella & wa & \\
\hline 987 & 鸟纲 & Aves & 雀形目 & Passeriformes & 太平鸟科 & Bombycillidae & 太平鸟 & Bombycilla garrulus & $\mathrm{cc}$ & \\
\hline 988 & 鸟纲 & Aves & 雀形目 & Passeriformes & 伯劳科 & Laniidae & 红背伯劳 & Lanius collurio & uf & \\
\hline
\end{tabular}


郜二虎, 何杰坤, 王志臣, 徐扬, 唐小平, 江海声. 全国陆生野生动物调查单元区划方案. 生物多样性, 2017, 25 (12): 1321-1330.

http://www.biodiversity-science.net/CN/10.17520/biods.2017135

\begin{tabular}{|c|c|c|c|c|c|c|c|c|c|c|}
\hline $\begin{array}{r}\text { 序号 } \\
\text { No. }\end{array}$ & \multicolumn{2}{|c|}{$\begin{array}{c}\text { 纲 } \\
\text { Class }\end{array}$} & \multicolumn{2}{|c|}{$\begin{array}{c}\text { 目 } \\
\text { Order }\end{array}$} & \multicolumn{2}{|r|}{$\begin{array}{c}\text { 科 } \\
\text { Family }\end{array}$} & \multicolumn{2}{|r|}{$\begin{array}{l}\text { 物种名 } \\
\text { Species }\end{array}$} & \multirow{2}{*}{$\begin{array}{l}\begin{array}{c}\text { 分布型 } \\
\text { Chorotype }\end{array} \\
x\end{array}$} & \multirow[t]{2}{*}{$\begin{array}{c}\text { 备注 } \\
\text { Remarks }\end{array}$} \\
\hline 989 & 鸟纲 & Aves & 雀形目 & Passeriformes & 伯劳科 & Laniidae & 红尾伯劳 & Lanius cristatus & & \\
\hline 990 & 鸟纲 & Aves & 雀形目 & Passeriformes & 伯劳科 & Laniidae & 栗背伯劳 & Lanius collurioides & wa & \\
\hline 991 & 鸟纲 & Aves & 雀形目 & Passeriformes & 伯劳科 & Laniidae & 棕背伯劳 & Lanius schach & wd & \\
\hline 992 & 鸟纲 & Aves & 雀形目 & Passeriformes & 伯劳科 & Laniidae & 灰背伯劳 & Lanius tephronotus & $\mathrm{hm}$ & \\
\hline 993 & 鸟纲 & Aves & 雀形目 & Passeriformes & 伯劳科 & Laniidae & 灰伯劳 & Lanius excubitor & $\mathrm{ch}$ & \\
\hline 994 & 鸟纲 & Aves & 雀形目 & Passeriformes & 伯劳科 & Laniidae & 黑额伯劳 & Lanius minor & 03 & \\
\hline 995 & 鸟纲 & Aves & 雀形目 & Passeriformes & 伯劳科 & Laniidae & 楔尾伯劳 & Lanius sphenocercus & $\mathrm{mc}$ & \\
\hline 996 & 鸟纲 & Aves & 雀形目 & Passeriformes & 盔鵙科 & Prionopidae & 钩嘴林鵙 & Tephrodornis gularis & $\mathrm{wb}$ & \\
\hline 997 & 鸟纲 & Aves & 雀形目 & Passeriformes & 黄鹏科 & Oriolidae & 金黄鹏 & Oriolus oriolus & o3 & \\
\hline 998 & 鸟纲 & Aves & 雀形目 & Passeriformes & 黄婯科 & Oriolidae & 黑头黄鹏 & Oriolus xanthornus & wa & \\
\hline 999 & 鸟纲 & Aves & 雀形目 & Passeriformes & 黄婯科 & Oriolidae & 朱鹏 & Oriolus traillii & wb & \\
\hline 1000 & 鸟纲 & Aves & 雀形目 & Passeriformes & 卷尾科 & Dicruridae & 鸦嘴卷尾 & Dicrurus annectans & wa & \\
\hline 1001 & 鸟纲 & Aves & 雀形目 & Passeriformes & 卷尾科 & Dicruridae & 古铜色卷尾 & Dicrurus aeneus & wa & \\
\hline 1002 & 鸟纲 & Aves & 雀形目 & Passeriformes & 卷尾科 & Dicruridae & 小盘尾 & Dicrurus remifer & wa & \\
\hline 1003 & 鸟纲 & Aves & 雀形目 & Passeriformes & 卷尾科 & Dicruridae & 大盘尾 & Dicrurus paradiseus & wa & \\
\hline 1004 & 鸟纲 & Aves & 雀形目 & Passeriformes & 椋鸟科 & Sturnidae & 金冠树八哥 & Ampeliceps coronatus & wa & \\
\hline 1005 & 鸟纲 & Aves & 雀形目 & Passeriformes & 椋鸟科 & Sturnidae & 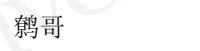 & Gracula religiosa & wa & \\
\hline 1006 & 鸟纲 & Aves & 雀形目 & Passeriformes & 椋鸟科 & Sturnidae & 林八哥 & Acridotheres grandis & wa & \\
\hline 1007 & 鸟纲 & Aves & 雀形目 & Passeriformes & 椋鸟科 & Sturnidae & 八哥 & Acridotheres cristatellus & wd & \\
\hline 1008 & 鸟纲 & Aves & 雀形目 & Passeriformes & 椋鸟科 & Sturnidae & 白领八哥 & Acridotheres albocinctus & wa & \\
\hline 1009 & 鸟纲 & Aves & 雀形目 & Passeriformes & 椋鸟科 & Sturnidae & 家八哥 & Acridotheres tristis & wd & \\
\hline 1010 & 鸟纲 & Aves & 雀形目 & Passeriformes & 椋鸟科 & Sturnidae & 红嘴椋鸟 & Acridotheres burmannicus & wa & \\
\hline
\end{tabular}


郜二虎, 何杰坤, 王志臣, 徐扬, 唐小平, 江海声. 全国陆生野生动物调查单元区划方案. 生物多样性, 2017, 25 (12): 1321-1330.

http://www.biodiversity-science.net/CN/10.17520/biods.2017135

\begin{tabular}{|c|c|c|c|c|c|c|c|c|c|c|}
\hline $\begin{array}{r}\text { 序号 } \\
\text { No. }\end{array}$ & \multicolumn{2}{|c|}{$\begin{array}{c}\text { 纲 } \\
\text { Class }\end{array}$} & \multicolumn{2}{|c|}{$\begin{array}{c}\text { 目 } \\
\text { Order }\end{array}$} & \multicolumn{2}{|r|}{$\begin{array}{c}\text { 科 } \\
\text { Family }\end{array}$} & \multicolumn{2}{|r|}{$\begin{array}{l}\text { 物种名 } \\
\text { Species }\end{array}$} & \multirow{2}{*}{$\begin{array}{l}\begin{array}{c}\text { 分布型 } \\
\text { Chorotype }\end{array} \\
\mathrm{ma}\end{array}$} & \multirow[t]{2}{*}{$\begin{array}{c}\text { 备注 } \\
\text { Remarks }\end{array}$} \\
\hline 1011 & 鸟纲 & Aves & 雀形目 & Passeriformes & 椋鸟科 & Sturnidae & 黑领椋鸟 & Gracupica nigricollis & & \\
\hline 1012 & 鸟纲 & Aves & 雀形目 & Passeriformes & 椋鸟科 & Sturnidae & 斑椋鸟 & Gracupica contra & wa & \\
\hline 1013 & 鸟纲 & Aves & 雀形目 & Passeriformes & 椋鸟科 & Sturnidae & 粉红椋鸟 & Pastor roseus & 03 & \\
\hline 1014 & 鸟纲 & Aves & 雀形目 & Passeriformes & 椋鸟科 & Sturnidae & 丝光椋鸟 & Sturnus sericeus & $\mathrm{sd}$ & \\
\hline 1015 & 鸟纲 & Aves & 雀形目 & Passeriformes & 椋鸟科 & Sturnidae & 灰椋鸟 & Sturnus cineraceus & $\mathrm{x}$ & \\
\hline 1016 & 鸟纲 & Aves & 雀形目 & Passeriformes & 椋鸟科 & Sturnidae & 紫翅椋鸟 & Sturnus vulgaris & 03 & \\
\hline 1017 & 鸟纲 & Aves & 雀形目 & Passeriformes & 燕鵙科 & Artamidae & 灰燕鵙 & Artamus fuscus & $\mathrm{wb}$ & \\
\hline 1018 & 鸟纲 & Aves & 雀形目 & Passeriformes & 鸦科 & Corvidae & 北噪鸦 & Perisoreus infaustus & uc & \\
\hline 1019 & 鸟纲 & Aves & 雀形目 & Passeriformes & 鸦科 & Corvidae & 黑头噪鸦 & Perisoreus internigrans & $\mathrm{pc}$ & \\
\hline 1020 & 鸟纲 & Aves & 雀形目 & Passeriformes & 鸦科 & Corvidae & 灰喜鹊 & Cyanopica cyanus & ud & \\
\hline 1021 & 鸟纲 & Aves & 雀形目 & Passeriformes & 鸦科 & Corvidae & 黄嘴蓝鹊 & Urocissa flavirostris & ha & \\
\hline 1022 & 鸟纲 & Aves & 雀形目 & Passeriformes & 鸦科 & Corvidae & 红嘴蓝鹊 & Urocissa erythrorhyncha & we & \\
\hline 1023 & 鸟纲 & Aves & 雀形目 & Passeriformes & 鸦科 & Corvidae & 白翅蓝鹊 & Urocissa whiteheadi & we & \\
\hline 1024 & 鸟纲 & Aves & 雀形目 & Passeriformes & 鸦科 & Corvidae & 蓝绿鹊 & Cissa chinensis & wa & \\
\hline 1025 & 鸟纲 & Aves & 雀形目 & Passeriformes & 鸦科 & Corvidae & 灰树鹊 & Dendrocitta formosae & wa & \\
\hline 1026 & 鸟纲 & Aves & 雀形目 & Passeriformes & 鸦科 & Corvidae & 黑额树鹊 & Dendrocitta frontalis & wa & \\
\hline 1027 & 鸟纲 & Aves & 雀形目 & Passeriformes & 鸦科 & Corvidae & 塔尾树鹊 & Temnurus temnurus & wa & \\
\hline 1028 & 鸟纲 & Aves & 雀形目 & Passeriformes & 鸦科 & Corvidae & 喜鹊 & Pica pica & $\mathrm{ch}$ & \\
\hline 1029 & 鸟纲 & Aves & 雀形目 & Passeriformes & 鸦科 & Corvidae & 黑尾地鸦 & Podoces hendersoni & $\mathrm{dg}$ & \\
\hline 1030 & 鸟纲 & Aves & 雀形目 & Passeriformes & 鸦科 & Corvidae & 白尾地鸦 & Podoces biddolphi & $\mathrm{db}$ & \\
\hline 1031 & 鸟纲 & Aves & 雀形目 & Passeriformes & 鸦科 & Corvidae & 星鸦 & Nucifraga caryocatactes & ue & \\
\hline 1032 & 鸟纲 & Aves & 雀形目 & Passeriformes & 鸦科 & Corvidae & 红嘴山鸦 & Pyrrhocorax pyrrhocorax & 03 & \\
\hline
\end{tabular}


郜二虎, 何杰坤, 王志臣, 徐扬, 唐小平, 江海声. 全国陆生野生动物调查单元区划方案. 生物多样性, 2017, 25 (12): 1321-1330.

http://www.biodiversity-science.net/CN/10.17520/biods.2017135

\begin{tabular}{|c|c|c|c|c|c|c|c|c|c|c|}
\hline $\begin{array}{r}\text { 序号 } \\
\text { No. }\end{array}$ & \multicolumn{2}{|c|}{$\begin{array}{c}\text { 纲 } \\
\text { Class }\end{array}$} & \multicolumn{2}{|c|}{$\begin{array}{c}\text { 目 } \\
\text { Order }\end{array}$} & \multicolumn{2}{|r|}{$\begin{array}{c}\text { 科 } \\
\text { Family }\end{array}$} & \multicolumn{2}{|r|}{$\begin{array}{l}\text { 物种名 } \\
\text { Species }\end{array}$} & \multirow{2}{*}{$\begin{array}{l}\begin{array}{c}\text { 分布型 } \\
\text { Chorotype }\end{array} \\
03\end{array}$} & \multirow[t]{2}{*}{$\begin{array}{c}\text { 备注 } \\
\text { Remarks }\end{array}$} \\
\hline 1033 & 鸟纲 & Aves & 雀形目 & Passeriformes & 鸦科 & Corvidae & 黄嘴山鸦 & Pyrrhocorax graculus & & \\
\hline 1034 & 鸟纲 & Aves & 雀形目 & Passeriformes & 鸦科 & Corvidae & 家鸦 & Corvus splendens & wa & \\
\hline 1035 & 鸟纲 & Aves & 雀形目 & Passeriformes & 鸦科 & Corvidae & 秃鼻乌鸦 & Corvus frugilegus & uf & \\
\hline 1036 & 鸟纲 & Aves & 雀形目 & Passeriformes & 鸦科 & Corvidae & 大嘴乌鸦 & Corvus macrorhynchos & eh & \\
\hline 1037 & 鸟纲 & Aves & 雀形目 & Passeriformes & 鸦科 & Corvidae & 白颈鸦 & Corvus pectoralis & sv & \\
\hline 1038 & 鸟纲 & Aves & 雀形目 & Passeriformes & 鸦科 & Corvidae & 渡鸦 & Corvus corax & $\mathrm{ch}$ & \\
\hline 1039 & 鸟纲 & Aves & 雀形目 & Passeriformes & 河乌科 & Cinclidae & 河乌 & Cinclus cinclus & o1 & \\
\hline 1040 & 鸟纲 & Aves & 雀形目 & Passeriformes & 河乌科 & Cinclidae & 褐河乌 & Cinclus pallasii & we & \\
\hline 1041 & 鸟纲 & Aves & 雀形目 & Passeriformes & 鹪鹑科 & Troglodytidae & 焦唵 & Troglodytes troglodytes & $\mathrm{ch}$ & \\
\hline 1042 & 鸟纲 & Aves & 雀形目 & Passeriformes & 岩翏科 & Prunellidae & 领岩翏 & Prunella collaris & ud & \\
\hline 1043 & 鸟纲 & Aves & 雀形目 & Passeriformes & 岩翏科 & Prunellidae & 高原岩鹨 & Prunella himalayana & $\mathrm{hb}$ & \\
\hline 1044 & 鸟纲 & Aves & 雀形目 & Passeriformes & 岩鹨科 & Prunellidae & 鸲岩鹨 & Prunella rubeculoides & $\mathrm{pd}$ & \\
\hline 1045 & 鸟纲 & Aves & 雀形目 & Passeriformes & 岩翏科 & Prunellidae & 棕胸岩翏 & Prunella strophiata & $\mathrm{hm}$ & \\
\hline 1046 & 鸟纲 & Aves & 雀形目 & Passeriformes & 岩翏科 & Prunellidae & 褐岩翏 & Prunella fulvescens & pw & \\
\hline 1047 & 鸟纲 & Aves & 雀形目 & Passeriformes & 岩翏科 & Prunellidae & 黑喉岩翏乌 & Prunella atrogularis & pw & \\
\hline 1048 & 鸟纲 & Aves & 雀形目 & Passeriformes & 岩翏科 & Prunellidae & 栗背岩鹨 & Prunella immaculata & hc & \\
\hline 1049 & 鸟纲 & Aves & 雀形目 & Passeriformes & 冻科 & Turdidae & 锈腹短翅冻 & Brachypteryx hyperythra & $\mathrm{hm}$ & \\
\hline 1050 & 鸟纲 & Aves & 雀形目 & Passeriformes & 冻科 & Turdidae & 栗背短翅鸫 & Brachypteryx stellata & $\mathrm{hm}$ & \\
\hline 1051 & 鸟纲 & Aves & 雀形目 & Passeriformes & 冻科 & Turdidae & 白喉短翅鸫 & Brachypteryx leucophrys & wc & \\
\hline 1052 & 鸟纲 & Aves & 雀形目 & Passeriformes & 冻科 & Turdidae & 蓝短翅鸫 & Brachypteryx montana & wd & \\
\hline 1053 & 鸟纲 & Aves & 雀形目 & Passeriformes & 冻科 & Turdidae & 新疆歌鸲 & Luscinia megarhynchos & o & \\
\hline 1054 & 鸟纲 & Aves & 雀形目 & Passeriformes & 冻科 & Turdidae & 红喉歌鸲 & Luscinia calliope & $\mathrm{u}$ & \\
\hline
\end{tabular}


郜二虎, 何杰坤, 王志臣, 徐扬, 唐小平, 江海声. 全国陆生野生动物调查单元区划方案. 生物多样性, 2017, 25 (12): 1321-1330.

http://www.biodiversity-science.net/CN/10.17520/biods.2017135

\begin{tabular}{|c|c|c|c|c|c|c|c|c|c|c|}
\hline $\begin{array}{r}\text { 序号 } \\
\text { No. }\end{array}$ & \multicolumn{2}{|c|}{$\begin{array}{c}\text { 纲 } \\
\text { Class }\end{array}$} & \multicolumn{2}{|c|}{$\begin{array}{c}\text { 目 } \\
\text { Order }\end{array}$} & \multicolumn{2}{|r|}{$\begin{array}{c}\text { 科 } \\
\text { Family }\end{array}$} & \multicolumn{2}{|r|}{$\begin{array}{l}\text { 物种名 } \\
\text { Species }\end{array}$} & \multirow{2}{*}{$\begin{array}{l}\begin{array}{c}\text { 分布型 } \\
\text { Chorotype }\end{array} \\
\mathrm{hm}\end{array}$} & \multirow[t]{2}{*}{$\begin{array}{c}\text { 备注 } \\
\text { Remarks }\end{array}$} \\
\hline 1055 & 鸟纲 & Aves & 雀形目 & Passeriformes & 冻科 & Turdidae & 黑胸歌鸲 & Luscinia pectoralis & & \\
\hline 1056 & 鸟纲 & Aves & 雀形目 & Passeriformes & 冻科 & Turdidae & 蓝.喉歌鸲 & Luscinia svecica & ua & \\
\hline 1057 & 鸟纲 & Aves & 雀形目 & Passeriformes & 冻科 & Turdidae & 棕头歌鸲 & Luscinia ruficeps & sv & \\
\hline 1058 & 鸟纲 & Aves & 雀形目 & Passeriformes & 冻科 & Turdidae & 黑喉歌鸲 & Luscinia obscura & sv & \\
\hline 1059 & 鸟纲 & Aves & 雀形目 & Passeriformes & 冻科 & Turdidae & 金胸歌鸲 & Luscinia pectardens & $\mathrm{hm}$ & \\
\hline 1060 & 鸟纲 & Aves & 雀形目 & Passeriformes & 冻科 & Turdidae & 金色林鸲 & Tarsiger chrysaeus & $\mathrm{hm}$ & \\
\hline 1061 & 鸟纲 & Aves & 雀形目 & Passeriformes & 冻科 & Turdidae & 白眉林鸲 & Tarsiger indicus & $\mathrm{hm}$ & \\
\hline 1062 & 鸟纲 & Aves & 雀形目 & Passeriformes & 冻科 & Turdidae & 棕腹林鸲 & Tarsiger hyperythrus & he & \\
\hline 1063 & 鸟纲 & Aves & 雀形目 & Passeriformes & 冻科 & Turdidae & 鹊鸲 & Copsychus saularis & wd & \\
\hline 1064 & 鸟纲 & Aves & 雀形目 & Passeriformes & 冻科 & Turdidae & 白腰鹊鸲 & Copsychus malabaricus & wa & \\
\hline 1065 & 鸟纲 & Aves & 雀形目 & Passeriformes & 冻科 & Turdidae & 红背红尾鸲 & Phoenicurus erythronotus & $\mathrm{dp}$ & \\
\hline 1066 & 鸟纲 & Aves & 雀形目 & Passeriformes & 冻科 & Turdidae & 蓝头红尾鸲 & Phoenicurus caeruleocephala & $\mathrm{dp}$ & \\
\hline 1067 & 鸟纲 & Aves & 雀形目 & Passeriformes & 冻科 & Turdidae & 赫红尾鸲 & Phoenicurus ochruros & o & \\
\hline 1068 & 鸟纲 & Aves & 雀形目 & Passeriformes & 冻科 & Turdidae & 黑喉红尾鸲 & Phoenicurus hodgsoni & $\mathrm{hm}$ & \\
\hline 1069 & 鸟纲 & Aves & 雀形目 & Passeriformes & 冻科 & Turdidae & 白喉红尾鸲 & Phoenicurus schisticeps & $\mathrm{hm}$ & \\
\hline 1070 & 鸟纲 & Aves & 雀形目 & Passeriformes & 冻科 & Turdidae & 红腹红尾鸲 & Phoenicurus erythrogastrus & $\mathrm{p}$ & \\
\hline 1071 & 鸟纲 & Aves & 雀形目 & Passeriformes & 冻科 & Turdidae & 蓝额红尾鸲 & Phoenicurus frontalis & $\mathrm{hm}$ & \\
\hline 1072 & 鸟纲 & Aves & 雀形目 & Passeriformes & 冻科 & Turdidae & 红尾水鸲 & Rhyacornis fuliginosa & we & \\
\hline 1073 & 鸟纲 & Aves & 雀形目 & Passeriformes & 冻科 & Turdidae & 白腹短翅鸲 & Hodgsonius phoenicuroides & $\mathrm{hm}$ & \\
\hline 1074 & 鸟纲 & Aves & 雀形目 & Passeriformes & 冻科 & Turdidae & 白尾地鸲 & Cinclidium leucurum & $\mathrm{hm}$ & \\
\hline 1075 & 鸟纲 & Aves & 雀形目 & Passeriformes & 冻科 & Turdidae & 蓝额地鸲 & Cinclidium frontale & $\mathrm{hm}$ & \\
\hline 1076 & 鸟纲 & Aves & 雀形目 & Passeriformes & 冻科 & Turdidae & 蓝大翅鸲 & Grandala coelicolor & $\mathrm{hm}$ & \\
\hline
\end{tabular}


郜二虎, 何杰坤, 王志臣, 徐扬, 唐小平, 江海声. 全国陆生野生动物调查单元区划方案. 生物多样性, 2017, 25 (12): 1321-1330.

http://www.biodiversity-science.net/CN/10.17520/biods.2017135

\begin{tabular}{|c|c|c|c|c|c|c|c|c|c|c|}
\hline \multirow{2}{*}{$\begin{array}{c}\text { 序号 } \\
\text { No. } \\
1077\end{array}$} & \multicolumn{2}{|c|}{$\begin{array}{c}\text { 纲 } \\
\text { Class }\end{array}$} & \multicolumn{2}{|c|}{$\begin{array}{c}\text { 目 } \\
\text { Order }\end{array}$} & \multicolumn{2}{|r|}{$\begin{array}{c}\text { 科 } \\
\text { Family }\end{array}$} & \multicolumn{2}{|r|}{$\begin{array}{l}\text { 物种名 } \\
\text { Species }\end{array}$} & \multirow{2}{*}{$\begin{array}{l}\text { 分布型 } \\
\text { Chorotype } \\
\text { sd }\end{array}$} & \multirow[t]{2}{*}{$\begin{array}{c}\text { 备注 } \\
\text { Remarks }\end{array}$} \\
\hline & 鸟纲 & Aves & 雀形目 & Passeriformes & 冻科 & Turdidae & 小燕尾 & Enicurus scouleri & & \\
\hline 1078 & 鸟纲 & Aves & 雀形目 & Passeriformes & 冻科 & Turdidae & 黑背燕尾 & Enicurus immaculatus & wd & \\
\hline 1079 & 鸟纲 & Aves & 雀形目 & Passeriformes & 冻科 & Turdidae & 灰背燕尾 & Enicurus schistaceus & wd & \\
\hline 1080 & 鸟纲 & Aves & 雀形目 & Passeriformes & 冻科 & Turdidae & 斑背燕尾 & Enicurus maculatus & $\mathrm{wc}$ & \\
\hline 1081 & 鸟纲 & Aves & 雀形目 & Passeriformes & 鸫科 & Turdidae & 紫宽嘴鸫 & Cochoa purpurea & sc & \\
\hline 1082 & 鸟纲 & Aves & 雀形目 & Passeriformes & 鸫科 & Turdidae & 绿宽嘴鸫 & Cochoa viridis & wa & \\
\hline 1083 & 鸟纲 & Aves & 雀形目 & Passeriformes & 鸫科 & Turdidae & 白斑黑石即鸟 & Saxicola caprata & wc & \\
\hline 1084 & 鸟纲 & Aves & 雀形目 & Passeriformes & 冻科 & Turdidae & 黑白林即鸟 & Saxicola jerdoni & wc & \\
\hline 1085 & 鸟纲 & Aves & 雀形目 & Passeriformes & 冻科 & Turdidae & 灰林即鸟 & Saxicola ferreus & wd & \\
\hline 1086 & 鸟纲 & Aves & 雀形目 & Passeriformes & 冻科 & Turdidae & 白背矶鸫 & Monticola saxatilis & da & \\
\hline 1087 & 鸟纲 & Aves & 雀形目 & Passeriformes & 冻科 & Turdidae & 栗腹矶冻 & Monticola rufiventris & sd & \\
\hline 1088 & 鸟纲 & Aves & 雀形目 & Passeriformes & 冻科 & Turdidae & 蓝矶东鸟 & Monticola solitarius & $\mathrm{u}$ & \\
\hline 1089 & 鸟纲 & Aves & 雀形目 & Passeriformes & 冻科 & Turdidae & 紫啸冻 & Myophonus caeruleus & we & \\
\hline 1090 & 鸟纲 & Aves & 雀形目 & Passeriformes & 冻科 & Turdidae & 橙头地鸫 & Zoothera citrina & wc & \\
\hline 1091 & 鸟纲 & Aves & 雀形目 & Passeriformes & 冻科 & Turdidae & 光背地冻 & Zoothera mollissima & $\mathrm{hm}$ & \\
\hline 1092 & 鸟纲 & Aves & 雀形目 & Passeriformes & 冻科 & Turdidae & 长尾地冻 & Zoothera dixoni & $\mathrm{hm}$ & \\
\hline 1093 & 鸟纲 & Aves & 雀形目 & Passeriformes & 冻科 & Turdidae & 虎斑地冻 & Zoothera dauma & $\mathrm{u}$ & \\
\hline 1094 & 鸟纲 & Aves & 雀形目 & Passeriformes & 冻科 & Turdidae & 长嘴地冻 & Zoothera marginata & wa & \\
\hline 1095 & 鸟纲 & Aves & 雀形目 & Passeriformes & 冻科 & Turdidae & 黑胸冻 & Turdus dissimilis & $\mathrm{hm}$ & \\
\hline 1096 & 鸟纲 & Aves & 雀形目 & Passeriformes & 冻科 & Turdidae & 白颈鸫 & Turdus albocinctus & ha & \\
\hline 1097 & 鸟纲 & Aves & 雀形目 & Passeriformes & 冻科 & Turdidae & 乌鸫 & Turdus merula & 03 & \\
\hline 1098 & 鸟纲 & Aves & 雀形目 & Passeriformes & 冻科 & Turdidae & 灰头鸫 & Turdus rubrocanus & $\mathrm{hm}$ & \\
\hline
\end{tabular}


郜二虎, 何杰坤, 王志臣, 徐扬, 唐小平, 江海声. 全国陆生野生动物调查单元区划方案. 生物多样性, 2017, 25 (12): 1321-1330.

http://www.biodiversity-science.net/CN/10.17520/biods.2017135

\begin{tabular}{|c|c|c|c|c|c|c|c|c|c|c|}
\hline $\begin{array}{r}\text { 序号 } \\
\text { No. }\end{array}$ & \multicolumn{2}{|c|}{$\begin{array}{c}\text { 纲 } \\
\text { Class }\end{array}$} & \multicolumn{2}{|c|}{$\begin{array}{c}\text { 目 } \\
\text { Order }\end{array}$} & \multicolumn{2}{|r|}{$\begin{array}{c}\text { 科 } \\
\text { Family }\end{array}$} & \multicolumn{2}{|r|}{$\begin{array}{l}\text { 物种名 } \\
\text { Species }\end{array}$} & \multirow{2}{*}{$\begin{array}{l}\begin{array}{c}\text { 分布型 } \\
\text { Chorotype }\end{array} \\
\mathrm{hm}\end{array}$} & \multirow[t]{2}{*}{$\begin{array}{c}\text { 备注 } \\
\text { Remarks }\end{array}$} \\
\hline 1099 & 鸟纲 & Aves & 雀形目 & Passeriformes & 冻科 & Turdidae & 棕背黑头冻 & Turdus kessleri & & \\
\hline 1100 & 鸟纲 & Aves & 雀形目 & Passeriformes & 冻科 & Turdidae & 褐头冻 & Turdus feae & we & \\
\hline 1101 & 鸟纲 & Aves & 雀形目 & Passeriformes & 冻科 & Turdidae & 白腹鸫 & Turdus pallidus & $\mathrm{mf}$ & \\
\hline 1102 & 鸟纲 & Aves & 雀形目 & Passeriformes & 冻科 & Turdidae & 赤颈冻 & Turdus ruficollis & o & \\
\hline 1103 & 鸟纲 & Aves & 雀形目 & Passeriformes & 冻科 & Turdidae & 田鸫 & Turdus pilaris & uc & \\
\hline 1104 & 鸟纲 & Aves & 雀形目 & Passeriformes & 冻科 & Turdidae & 宝兴歌鸫 & Turdus mupinensis & hc & \\
\hline 1105 & 鸟纲 & Aves & 雀形目 & Passeriformes & 冻科 & Turdidae & 葪冻 & Turdus viscivorus & o & \\
\hline 1106 & 鸟纲 & Aves & 雀形目 & Passeriformes & 姾科 & Muscicapidae & 白喉姬暡 & Ficedula monileger & wa & \\
\hline 1107 & 鸟纲 & Aves & 雀形目 & Passeriformes & 姾科 & Muscicapidae & 小斑姬暡 & Ficedula westermanni & $\mathrm{wb}$ & \\
\hline 1108 & 鸟纲 & Aves & 雀形目 & Passeriformes & 姾科 & Muscicapidae & 玉头姬暡 & Ficedula sapphira & $\mathrm{hm}$ & \\
\hline 1109 & 鸟纲 & Aves & 雀形目 & Passeriformes & 姾科 & Muscicapidae & 铜蓝鹟 & Eumyias thalassinus & wd & \\
\hline 1110 & 鸟纲 & Aves & 雀形目 & Passeriformes & 姾科 & Muscicapidae & 大仙暡 & Niltava grandis & wa & \\
\hline 1111 & 鸟纲 & Aves & 雀形目 & Passeriformes & 姾科 & Muscicapidae & 小仙暡 & Niltava macgrigoriae & $\mathrm{hm}$ & \\
\hline 1112 & 鸟纲 & Aves & 雀形目 & Passeriformes & 蜍科 & Muscicapidae & 棕腹大仙稻 & Niltava davidi & wa & \\
\hline 1113 & 鸟纲 & Aves & 雀形目 & Passeriformes & 叙科 & Muscicapidae & 棕腹仙鹟 & Niltava sundara & $\mathrm{hm}$ & \\
\hline 1114 & 鸟纲 & Aves & 雀形目 & Passeriformes & 姾科 & Muscicapidae & 棕腹蓝仙䳜 & Niltava vivida & $\mathrm{hm}$ & \\
\hline 1115 & 鸟纲 & Aves & 雀形目 & Passeriformes & 姾科 & Muscicapidae & 白尾蓝仙䳜 & Cyornis concretus & wa & \\
\hline 1116 & 鸟纲 & Aves & 雀形目 & Passeriformes & 姾科 & Muscicapidae & 海南蓝仙鹟 & Cyornis hainanus & $\mathrm{sb}$ & \\
\hline 1117 & 鸟纲 & Aves & 雀形目 & Passeriformes & 姾科 & Muscicapidae & 灰颊仙鹟 & Cyornis poliogenys & wa & \\
\hline 1118 & 鸟纲 & Aves & 雀形目 & Passeriformes & 姾科 & Muscicapidae & 纯蓝仙鹟 & Cyornis unicolor & wb & \\
\hline 1119 & 鸟纲 & Aves & 雀形目 & Passeriformes & 姾科 & Muscicapidae & 山蓝仙䳜 & Cyornis banyumas & wb & \\
\hline 1120 & 鸟纲 & Aves & 雀形目 & Passeriformes & 㪘科 & Muscicapidae & 侏蓝仙蜍 & Muscicapella hodgsoni & wa & \\
\hline
\end{tabular}


郜二虎, 何杰坤, 王志臣, 徐扬, 唐小平, 江海声. 全国陆生野生动物调查单元区划方案. 生物多样性, 2017, 25 (12): 1321-1330.

http://www.biodiversity-science.net/CN/10.17520/biods.2017135

\begin{tabular}{|c|c|c|c|c|c|c|c|c|c|c|}
\hline $\begin{array}{r}\text { 序号 } \\
\text { No. }\end{array}$ & \multicolumn{2}{|c|}{$\begin{array}{c}\text { 纲 } \\
\text { Class }\end{array}$} & \multicolumn{2}{|c|}{$\begin{array}{c}\text { 目 } \\
\text { Order }\end{array}$} & \multicolumn{2}{|r|}{$\begin{array}{c}\text { 科 } \\
\text { Family }\end{array}$} & \multicolumn{2}{|r|}{$\begin{array}{l}\text { 物种名 } \\
\text { Species }\end{array}$} & \multirow{2}{*}{$\begin{array}{l}\begin{array}{c}\text { 分布型 } \\
\text { Chorotype }\end{array} \\
\mathrm{hm}\end{array}$} & \multirow[t]{2}{*}{$\begin{array}{c}\text { 备注 } \\
\text { Remarks }\end{array}$} \\
\hline 1121 & 鸟纲 & Aves & 雀形目 & Passeriformes & 扇尾䳜科 & Rhipiduridae & 黄腹扇尾鹟 & Rhipidura hypoxantha & & \\
\hline 1122 & 鸟纲 & Aves & 雀形目 & Passeriformes & 扇尾鹟科 & Rhipiduridae & 白喉扇尾众 & Rhipidura albicollis & wc & \\
\hline 1123 & 鸟纲 & Aves & 雀形目 & Passeriformes & 扇尾鹟科 & Rhipiduridae & 白眉扇尾暡 & Rhipidura aureola & $\mathrm{w}$ & \\
\hline 1124 & 鸟纲 & Aves & 雀形目 & Passeriformes & 画眉科 & Timaliidae & 黑脸噪倜 & Garrulax perspicillatus & sd & \\
\hline 1125 & 鸟纲 & Aves & 雀形目 & Passeriformes & 画眉科 & Timaliidae & 白喉噪鹋 & Garrulax albogularis & $\mathrm{hm}$ & \\
\hline 1126 & 鸟纲 & Aves & 雀形目 & Passeriformes & 画眉科 & Timaliidae & 白冠噪鹛 & Garrulax leucolophus & wa & \\
\hline 1127 & 鸟纲 & Aves & 雀形目 & Passeriformes & 画眉科 & Timaliidae & 小黑领噪倜 & Garrulax monileger & $\mathrm{wb}$ & \\
\hline 1128 & 鸟纲 & Aves & 雀形目 & Passeriformes & 画眉科 & Timaliidae & 黑领噪鹤 & Garrulax pectoralis & wd & \\
\hline 1129 & 鸟纲 & Aves & 雀形目 & Passeriformes & 画眉科 & Timaliidae & 条纹噪鹤 & Garrulax striatus & $\mathrm{hm}$ & \\
\hline 1130 & 鸟纲 & Aves & 雀形目 & Passeriformes & 画眉科 & Timaliidae & 白颈噪鵰 & Garrulax strepitans & wa & \\
\hline 1131 & 鸟纲 & Aves & 雀形目 & Passeriformes & 画眉科 & Timaliidae & 褐胸噪柅鸟 & Garrulax maesi & $\mathrm{sc}$ & \\
\hline 1132 & 鸟纲 & Aves & 雀形目 & Passeriformes & 画眉科 & Timaliidae & 栗颈噪鹊 & Garrulax ruficollis & $\mathrm{hm}$ & \\
\hline 1133 & 鸟纲 & Aves & 雀形目 & Passeriformes & 画眉科 & Timaliidae & 黑喉噪鹋 & Garrulax chinensis & wa & \\
\hline 1134 & 鸟纲 & Aves & 雀形目 & Passeriformes & 画眉科 & Timaliidae & 山噪倜 & Garrulax davidi & ba & \\
\hline 1135 & 鸟纲 & Aves & 雀形目 & Passeriformes & 画眉科 & Timaliidae & 黑额山噪倜 & Garrulax sukatschewi & $\mathrm{pf}$ & \\
\hline 1136 & 鸟纲 & Aves & 雀形目 & Passeriformes & 画眉科 & Timaliidae & 灰翅噪眮 & Garrulax cineraceus & sv & \\
\hline 1137 & 鸟纲 & Aves & 雀形目 & Passeriformes & 画眉科 & Timaliidae & 眼纹噪鹏 & Garrulax ocellatus & $\mathrm{hm}$ & \\
\hline 1138 & 鸟纲 & Aves & 雀形目 & Passeriformes & 画眉科 & Timaliidae & 斑背噪鹛 & Garrulax lunulatus & hc & \\
\hline 1139 & 鸟纲 & Aves & 雀形目 & Passeriformes & 画眉科 & Timaliidae & 大噪倜 & Garrulax maximus & hc & \\
\hline 1140 & 鸟纲 & Aves & 雀形目 & Passeriformes & 画眉科 & Timaliidae & 灰胁噪哃鸟 & Garrulax caerulatus & $\mathrm{hm}$ & \\
\hline 1141 & 鸟纲 & Aves & 雀形目 & Passeriformes & 画眉科 & Timaliidae & 棕噪峟 & Garrulax poecilorhynchus & $\mathrm{sc}$ & \\
\hline 1142 & 鸟纲 & Aves & 雀形目 & Passeriformes & 画眉科 & Timaliidae & 斑胸噪鸲 & Garrulax merulinus & wd & \\
\hline
\end{tabular}


郜二虎, 何杰坤, 王志臣, 徐扬, 唐小平, 江海声. 全国陆生野生动物调查单元区划方案. 生物多样性, 2017, 25 (12): 1321-1330.

http://www.biodiversity-science.net/CN/10.17520/biods.2017135

\begin{tabular}{|c|c|c|c|c|c|c|c|c|c|c|}
\hline $\begin{array}{r}\text { 序号 } \\
\text { No. }\end{array}$ & \multicolumn{2}{|c|}{$\begin{array}{c}\text { 纲 } \\
\text { Class }\end{array}$} & \multicolumn{2}{|c|}{$\begin{array}{c}\text { 目 } \\
\text { Order }\end{array}$} & \multicolumn{2}{|r|}{$\begin{array}{c}\text { 科 } \\
\text { Family }\end{array}$} & \multicolumn{2}{|r|}{$\begin{array}{l}\text { 物种名 } \\
\text { Species }\end{array}$} & \multirow{2}{*}{$\begin{array}{l}\begin{array}{c}\text { 分布型 } \\
\text { Chorotype }\end{array} \\
\text { sd }\end{array}$} & \multirow[t]{2}{*}{$\begin{array}{c}\text { 备注 } \\
\text { Remarks }\end{array}$} \\
\hline 1143 & 鸟纲 & Aves & 雀形目 & Passeriformes & 画眉科 & Timaliidae & 画眉 & Garrulax canorus & & \\
\hline 1144 & 鸟纲 & Aves & 雀形目 & Passeriformes & 画眉科 & Timaliidae & 白项噪鹏 & Garrulax sannio & sd & \\
\hline 1145 & 鸟纲 & Aves & 雀形目 & Passeriformes & 画眉科 & Timaliidae & 细纹噪鹊 & Garrulax lineatus & ha & \\
\hline 1146 & 鸟纲 & Aves & 雀形目 & Passeriformes & 画眉科 & Timaliidae & 纯色噪鹏 & Garrulax subunicolor & $\mathrm{hm}$ & \\
\hline 1147 & 鸟纲 & Aves & 雀形目 & Passeriformes & 画眉科 & Timaliidae & 蓝翅噪鸬 & Garrulax squamatus & $\mathrm{hm}$ & \\
\hline 1148 & 鸟纲 & Aves & 雀形目 & Passeriformes & 画眉科 & Timaliidae & 橙翅噪鹛 & Garrulax elliotii & hc & \\
\hline 1149 & 鸟纲 & Aves & 雀形目 & Passeriformes & 画眉科 & Timaliidae & 杂色噪倜 & Garrulax variegatus & ha & \\
\hline 1150 & 鸟纲 & Aves & 雀形目 & Passeriformes & 画眉科 & Timaliidae & 灰腹噪鹛 & Grrulax henrici & hd & \\
\hline 1151 & 鸟纲 & Aves & 雀形目 & Passeriformes & 画眉科 & Timaliidae & 黑顶噪鸬 & Garrulax affinis & $\mathrm{hm}$ & \\
\hline 1152 & 鸟纲 & Aves & 雀形目 & Passeriformes & 画眉科 & Timaliidae & 红头噪䲿 & Garrulax erythrocephalus & $\mathrm{hm}$ & \\
\hline 1153 & 鸟纲 & Aves & 雀形目 & Passeriformes & 画眉科 & Timaliidae & 红翅噪屇 & Garrulax formosus & hc & \\
\hline 1154 & 鸟纲 & Aves & 雀形目 & Passeriformes & 画眉科 & Timaliidae & 红尾噪倜 & Garrulax milnei & wc & \\
\hline 1155 & 鸟纲 & Aves & 雀形目 & Passeriformes & 画眉科 & Timaliidae & 灰胸薮柅鸟 & Liocichla omeiensis & hc & \\
\hline 1156 & 鸟纲 & Aves & 雀形目 & Passeriformes & 画眉科 & Timaliidae & 红翅薮䧓 & Liocichla phoenicea & $\mathrm{hm}$ & \\
\hline 1157 & 鸟纲 & Aves & 雀形目 & Passeriformes & 画眉科 & Timaliidae & 棕胸幽鹛 & Pellorneum tickelli & wa & \\
\hline 1158 & 鸟纲 & Aves & 雀形目 & Passeriformes & 画眉科 & Timaliidae & 白腹幽扸 & Pellorneum albiventre & wa & \\
\hline 1159 & 鸟纲 & Aves & 雀形目 & Passeriformes & 画眉科 & Timaliidae & 棕头幽扸 & Pellorneum ruficeps & wa & \\
\hline 1160 & 鸟纲 & Aves & 雀形目 & Passeriformes & 画眉科 & Timaliidae & 长嘴钩嘴勆 & Pomatorhlnus hypoleucos & $\mathrm{wb}$ & \\
\hline 1161 & 鸟纲 & Aves & 雀形目 & Passeriformes & 画眉科 & Timaliidae & 斑胸钩嘴煟 & Pomatorhinus erythrocnemis & sd & \\
\hline 1162 & 鸟纲 & Aves & 雀形目 & Passeriformes & 画眉科 & Timaliidae & 棕颈钩嘴倜 & Pomatorhinus rujficollis & wa & \\
\hline 1163 & 鸟纲 & Aves & 雀形目 & Passeriformes & 画眉科 & Timaliidae & 棕头钩嘴扸 & Pomatorhinus ochraceiceps & wa & \\
\hline 1164 & 鸟纲 & Aves & 雀形目 & Passeriformes & 画眉科 & Timaliidae & 红嘴钩嘴鵰 & Pomatorhinus ferruginosus & wa & \\
\hline
\end{tabular}


郜二虎, 何杰坤, 王志臣, 徐扬, 唐小平, 江海声. 全国陆生野生动物调查单元区划方案. 生物多样性, 2017, 25 (12): 1321-1330.

http://www.biodiversity-science.net/CN/10.17520/biods.2017135

\begin{tabular}{|c|c|c|c|c|c|c|c|c|c|c|}
\hline $\begin{array}{r}\text { 序号 } \\
\text { No. }\end{array}$ & \multicolumn{2}{|c|}{$\begin{array}{c}\text { 纲 } \\
\text { Class }\end{array}$} & \multicolumn{2}{|c|}{$\begin{array}{c}\text { 目 } \\
\text { Order }\end{array}$} & \multicolumn{2}{|r|}{$\begin{array}{c}\text { 科 } \\
\text { Family }\end{array}$} & \multicolumn{2}{|r|}{$\begin{array}{l}\text { 物种名 } \\
\text { Species }\end{array}$} & \multirow{2}{*}{$\begin{array}{l}\begin{array}{c}\text { 分布型 } \\
\text { Chorotype }\end{array} \\
\text { wa }\end{array}$} & \multirow[t]{2}{*}{$\begin{array}{c}\text { 备注 } \\
\text { Remarks }\end{array}$} \\
\hline 1165 & 鸟纲 & Aves & 雀形目 & Passeriformes & 画眉科 & Timaliidae & 剑嘴鹤 & Xiphirhynchus superciliaris & & \\
\hline 1166 & 鸟纲 & Aves & 雀形目 & Passeriformes & 画眉科 & Timaliidae & 灰岩鹪扸 & Napothera crispifrons & wa & \\
\hline 1167 & 鸟纲 & Aves & 雀形目 & Passeriformes & 画眉科 & Timaliidae & 短尾鹪鹛 & Napothera brevicaudata & wa & \\
\hline 1168 & 鸟纲 & Aves & 雀形目 & Passeriformes & 画眉科 & Timaliidae & 纹胸鹪鹛 & Napothera epilepidota & wa & \\
\hline 1169 & 鸟纲 & Aves & 雀形目 & Passeriformes & 画眉科 & Timaliidae & 鳞胸鹪鹛 & Pnoepyga abliventer & $\mathrm{hm}$ & \\
\hline 1170 & 鸟纲 & Aves & 雀形目 & Passeriformes & 画眉科 & Timaliidae & 小鳞胸鹪鹤 & Pnoepyga pusilla & wd & \\
\hline 1171 & 鸟纲 & Aves & 雀形目 & Passeriformes & 画眉科 & Timaliidae & 斑翅絜䴗 & Spelaeornis troglodytoides & $\mathrm{hm}$ & \\
\hline 1172 & 鸟纲 & Aves & 雀形目 & Passeriformes & 画眉科 & Timaliidae & 丽星鸮鹛 & Spelaeornis formosus & $\mathrm{sb}$ & \\
\hline 1173 & 鸟纲 & Aves & 雀形目 & Passeriformes & 画眉科 & Timaliidae & 长尾鸽柅 & Spelaeornis chocolatinus & $\mathrm{wb}$ & \\
\hline 1174 & 鸟纲 & Aves & 雀形目 & Passeriformes & 画眉科 & Timaliidae & 黄喉穗柅鸟 & Stachyris ambigua & $\mathrm{hm}$ & \\
\hline 1175 & 鸟纲 & Aves & 雀形目 & Passeriformes & 画眉科 & Timaliidae & 红头穗扸 & Stachyris ruficeps & sd & \\
\hline 1176 & 鸟纲 & Aves & 雀形目 & Passeriformes & 画眉科 & Timaliidae & 金头穗僱 & Stachyris chrysaea & wa & \\
\hline 1177 & 鸟纲 & Aves & 雀形目 & Passeriformes & 画眉科 & Timaliidae & 黑头穗鹛 & Stachyris nigriceps & wa & \\
\hline 1178 & 鸟纲 & Aves & 雀形目 & Passeriformes & 画眉科 & Timaliidae & 斑颈穗哃 & Stachyris striolata & wa & \\
\hline 1179 & 鸟纲 & Aves & 雀形目 & Passeriformes & 画眉科 & Timaliidae & 纹胸䴗 & Macronous gularis & wa & \\
\hline 1180 & 鸟纲 & Aves & 雀形目 & Passeriformes & 画眉科 & Timaliidae & 红顶鹋 & Timalia pileata & wb & \\
\hline 1181 & 鸟纲 & Aves & 雀形目 & Passeriformes & 画眉科 & Timaliidae & 金眼哃雀 & Chrysommd sinense & wb & \\
\hline 1182 & 鸟纲 & Aves & 雀形目 & Passeriformes & 画眉科 & Timaliidae & 宝兴哃雀 & Moupinia poecilotis & hc & \\
\hline 1183 & 鸟纲 & Aves & 雀形目 & Passeriformes & 画眉科 & Timaliidae & 矛纹草哃 & Babax lanceolatus & sd & \\
\hline 1184 & 鸟纲 & Aves & 雀形目 & Passeriformes & 画眉科 & Timaliidae & 大草鹛 & Babax waddelli & $\mathrm{pd}$ & \\
\hline 1185 & 鸟纲 & Aves & 雀形目 & Passeriformes & 画眉科 & Timaliidae & 棕草柅鸟 & Babax koslowi & hc & \\
\hline 1186 & 鸟纲 & Aves & 雀形目 & Passeriformes & 画眉科 & Timaliidae & 银耳相思鸟 & Leiothrix argentauris & wc & \\
\hline
\end{tabular}


郜二虎, 何杰坤, 王志臣, 徐扬, 唐小平, 江海声. 全国陆生野生动物调查单元区划方案. 生物多样性, 2017, 25 (12): 1321-1330.

http://www.biodiversity-science.net/CN/10.17520/biods.2017135

\begin{tabular}{|c|c|c|c|c|c|c|c|c|c|c|}
\hline \multirow{2}{*}{$\begin{array}{c}\text { 序号 } \\
\text { No. } \\
1187\end{array}$} & \multicolumn{2}{|c|}{$\begin{array}{c}\text { 纲 } \\
\text { Class }\end{array}$} & \multicolumn{2}{|c|}{$\begin{array}{c}\text { 目 } \\
\text { Order }\end{array}$} & \multirow{2}{*}{\multicolumn{2}{|c|}{$\begin{array}{c}\text { 科 } \\
\text { Family } \\
\text { Timaliidae }\end{array}$}} & \multicolumn{2}{|r|}{$\begin{array}{l}\text { 物种名 } \\
\text { Species }\end{array}$} & \multirow{2}{*}{\begin{tabular}{|l}
\multicolumn{1}{|c|}{$\begin{array}{c}\text { 分布型 } \\
\text { Chorotype }\end{array}$} \\
wd
\end{tabular}} & \multirow[t]{2}{*}{$\begin{array}{c}\text { 备注 } \\
\text { Remarks }\end{array}$} \\
\hline & 鸟纲 & Aves & 雀形目 & Passeriformes & & & 红嘴相思鸟 & Leiothrix lutea & & \\
\hline 1188 & 鸟纲 & Aves & 雀形目 & Passeriformes & 画眉科 & Timaliidae & 斑胁姬柅⿹ & Curia nipalensis & $\mathrm{hm}$ & \\
\hline 1189 & 鸟纲 & Aves & 雀形目 & Passeriformes & 画眉科 & Timaliidae & 棕腹鵙鹛 & Ptcruthius rufiventer & $\mathrm{hm}$ & \\
\hline 1190 & 鸟纲 & Aves & 雀形目 & Passeriformes & 画眉科 & Timaliidae & 红翅鵙扸 & Pteruthius flaviscapis & wc & \\
\hline 1191 & 鸟纲 & Aves & 雀形目 & Passeriformes & 画眉科 & Timaliidae & 淡绿鵙倜 & Pteruthius xanthochlorus & $\mathrm{hm}$ & \\
\hline 1192 & 鸟纲 & Aves & 雀形目 & Passeriformes & 画眉科 & Timaliidae & 栗喉鵙鹛 & Pteruthius melanotis & wa & \\
\hline 1193 & 鸟纲 & Aves & 雀形目 & Passeriformes & 画眉科 & Timaliidae & 栗额鵙扸 & Pteruthius aenobarbus & wb & \\
\hline 1194 & 鸟纲 & Aves & 雀形目 & Passeriformes & 画眉科 & Timaliidae & 白头鵙鸪 & Gampsorhynchus rafulus & wa & \\
\hline 1195 & 鸟纲 & Aves & 雀形目 & Passeriformes & 画眉科 & Timaliidae & 栗额斑翅鶥 & Actinodura egertoni & wa & \\
\hline 1196 & 鸟纲 & Aves & 雀形目 & Passeriformes & 画眉科 & Timaliidae & 白眶斑翅鵰 & Actinodura ramsayi & wa & \\
\hline 1197 & 鸟纲 & Aves & 雀形目 & Passeriformes & 画眉科 & Timaliidae & 纹头斑翅鹋 & Actinodura nipalensis & ha & \\
\hline 1198 & 鸟纲 & Aves & 雀形目 & Passeriformes & 画眉科 & Timaliidae & 纹胸斑翅鵰 & Actinodura waldeni & he & \\
\hline 1199 & 鸟纲 & Aves & 雀形目 & Passeriformes & 画眉科 & Timaliidae & 灰头斑翅䴗 & Actinodura souliei & hc & \\
\hline 1200 & 鸟纲 & Aves & 雀形目 & Passeriformes & 画眉科 & Timaliidae & 蓝翅希鹛 & Minla cyanouroptera & $\mathrm{wc}$ & \\
\hline 1201 & 鸟纲 & Aves & 雀形目 & Passeriformes & 画眉科 & Timaliidae & 斑喉希䴗 & Minla strigula & $\mathrm{hm}$ & \\
\hline 1202 & 鸟纲 & Aves & 雀形目 & Passeriformes & 画眉科 & Timaliidae & 红尾希煟 & Minla ignotincta & $\mathrm{sc}$ & \\
\hline 1203 & 鸟纲 & Aves & 雀形目 & Passeriformes & 画眉科 & Timaliidae & 金胸雀䴗 & Alcippe chrysotis & $\mathrm{hm}$ & \\
\hline 1204 & 鸟纲 & Aves & 雀形目 & Passeriformes & 画眉科 & Timaliidae & 金额雀倜 & Alcippe variegaticeps & $\mathrm{s}$ & \\
\hline 1205 & 鸟纲 & Aves & 雀形目 & Passeriformes & 画眉科 & Timaliidae & 黄喉雀倜 & Alcippe cinerea & $\mathrm{hm}$ & \\
\hline 1206 & 鸟纲 & Aves & 雀形目 & Passeriformes & 画眉科 & Timaliidae & 栗头雀鸤 & Alcippe castaneceps & wa & \\
\hline 1207 & 鸟纲 & Aves & 雀形目 & Passeriformes & 画眉科 & Timaliidae & 白眉雀鸤 & Alcippe vinipectus & $\mathrm{hm}$ & \\
\hline 1208 & 鸟纲 & Aves & 雀形目 & Passeriformes & 画眉科 & Timaliidae & 中华雀鹋 & Alcippe striaticollis & $\mathrm{hc}$ & \\
\hline
\end{tabular}


郜二虎, 何杰坤, 王志臣, 徐扬, 唐小平, 江海声. 全国陆生野生动物调查单元区划方案. 生物多样性, 2017, 25 (12): 1321-1330.

http://www.biodiversity-science.net/CN/10.17520/biods.2017135

\begin{tabular}{|c|c|c|c|c|c|c|c|c|c|c|}
\hline $\begin{array}{r}\text { 序号 } \\
\text { No. }\end{array}$ & \multicolumn{2}{|c|}{$\begin{array}{c}\text { 纲 } \\
\text { Class }\end{array}$} & \multicolumn{2}{|c|}{$\begin{array}{c}\text { 目 } \\
\text { Order }\end{array}$} & \multicolumn{2}{|r|}{$\begin{array}{c}\text { 科 } \\
\text { Family }\end{array}$} & \multicolumn{2}{|r|}{$\begin{array}{l}\text { 物种名 } \\
\text { Species }\end{array}$} & \multirow{2}{*}{$\begin{array}{l}\begin{array}{c}\text { 分布型 } \\
\text { Chorotype }\end{array} \\
\text { hc }\end{array}$} & \multirow[t]{2}{*}{$\begin{array}{c}\text { 备注 } \\
\text { Remarks }\end{array}$} \\
\hline 1209 & 鸟纲 & Aves & 雀形目 & Passeriformes & 画眉科 & Timaliidae & 棕头雀倜 & Alcippe ruficapilla & & \\
\hline 1210 & 鸟纲 & Aves & 雀形目 & Passeriformes & 画眉科 & Timaliidae & 褐头雀倜 & Alcippe cinereiceps & sd & \\
\hline 1211 & 鸟纲 & Aves & 雀形目 & Passeriformes & 画眉科 & Timaliidae & 棕喉雀倜 & Alcippe rufogularis & $\mathrm{hm}$ & \\
\hline 1212 & 鸟纲 & Aves & 雀形目 & Passeriformes & 画眉科 & Timaliidae & 褐胁雀倜 & Alcippe dubia & wc & \\
\hline 1213 & 鸟纲 & Aves & 雀形目 & Passeriformes & 画眉科 & Timaliidae & 褐顶雀倜 & Alcippe brunnea & wd & \\
\hline 1214 & 鸟纲 & Aves & 雀形目 & Passeriformes & 画眉科 & Timaliidae & 褐脸雀倜 & Alcippe poioicephala & wa & \\
\hline 1215 & 鸟纲 & Aves & 雀形目 & Passeriformes & 画眉科 & Timaliidae & 灰眶雀鸲 & Alcippe morrisonia & wd & \\
\hline 1216 & 鸟纲 & Aves & 雀形目 & Passeriformes & 画眉科 & Timaliidae & 白眶雀鹇 & Alcippe nipalensis & he & \\
\hline 1217 & 鸟纲 & Aves & 雀形目 & Passeriformes & 画眉科 & Timaliidae & 栗背奇倜 & Heterophasia annectens & $\mathrm{hm}$ & \\
\hline 1218 & 鸟纲 & Aves & 雀形目 & Passeriformes & 画眉科 & Timaliidae & 灰奇鹤 & Heterophasia gracilis & he & \\
\hline 1219 & 鸟纲 & Aves & 雀形目 & Passeriformes & 画眉科 & Timaliidae & 黑头奇鹋 & Heterophasia melanoleuca & ha & \\
\hline 1220 & 鸟纲 & Aves & 雀形目 & Passeriformes & 画眉科 & Timaliidae & 丽色奇倜 & Heterophasia pulchella & he & \\
\hline 1221 & 鸟纲 & Aves & 雀形目 & Passeriformes & 画眉科 & Timaliidae & 长尾奇鹋 & Heterophasia picaoides & wa & \\
\hline 1222 & 鸟纲 & Aves & 雀形目 & Passeriformes & 画眉科 & Timaliidae & 栗耳风倜 & Yuhina castaniceps & wc & \\
\hline 1223 & 鸟纲 & Aves & 雀形目 & Passeriformes & 画眉科 & Timaliidae & 白颈风倜 & Yuhina bakeri & he & \\
\hline 1224 & 鸟纲 & Aves & 雀形目 & Passeriformes & 画眉科 & Timaliidae & 黄颈风倜 & Yuhina flavicollis & $\mathrm{hm}$ & \\
\hline 1225 & 鸟纲 & Aves & 雀形目 & Passeriformes & 画眉科 & Timaliidae & 纹喉风倜 & Yuhina gularis & $\mathrm{hm}$ & \\
\hline 1226 & 鸟纲 & Aves & 雀形目 & Passeriformes & 画眉科 & Timaliidae & 白领风倜 & Yuhina diademata & hc & \\
\hline 1227 & 鸟纲 & Aves & 雀形目 & Passeriformes & 画眉科 & Timaliidae & 棕薢风倜 & Yuhina occipitalis & $\mathrm{hm}$ & \\
\hline 1228 & 鸟纲 & Aves & 雀形目 & Passeriformes & 画眉科 & Timaliidae & 黑㤥风倜 & Yuhina nigrimenta & wc & \\
\hline 1229 & 鸟纲 & Aves & 雀形目 & Passeriformes & 画眉科 & Timaliidae & 白腹风倜 & Erpornis zantholeuca & wb & \\
\hline 1230 & 鸟纲 & Aves & 雀形目 & Passeriformes & 画眉科 & Timaliidae & 火尾绿倜 & Myzornis pyrrhoura & $\mathrm{hm}$ & \\
\hline
\end{tabular}


郜二虎, 何杰坤, 王志臣, 徐扬, 唐小平, 江海声. 全国陆生野生动物调查单元区划方案. 生物多样性, 2017, 25 (12): 1321-1330.

http://www.biodiversity-science.net/CN/10.17520/biods.2017135

\begin{tabular}{|c|c|c|c|c|c|c|c|c|c|c|}
\hline $\begin{array}{r}\text { 序号 } \\
\text { No. }\end{array}$ & \multicolumn{2}{|c|}{$\begin{array}{c}\text { 纲 } \\
\text { Class }\end{array}$} & \multicolumn{2}{|c|}{$\begin{array}{c}\text { 目 } \\
\text { Order }\end{array}$} & \multicolumn{2}{|r|}{$\begin{array}{c}\text { 科 } \\
\text { Family }\end{array}$} & \multicolumn{2}{|r|}{$\begin{array}{l}\text { 物种名 } \\
\text { Species }\end{array}$} & \multirow{2}{*}{$\begin{array}{l}\begin{array}{c}\text { 分布型 } \\
\text { Chorotype }\end{array} \\
\mathrm{hm}\end{array}$} & \multirow[t]{2}{*}{$\begin{array}{c}\text { 备注 } \\
\text { Remarks }\end{array}$} \\
\hline 1231 & 鸟纲 & Aves & 雀形目 & Passeriformes & 鸦雀科 & Paradoxornithidae & 红嘴鸦雀 & Conostoma oemodium & & \\
\hline 1232 & 鸟纲 & Aves & 雀形目 & Passeriformes & 鸦雀科 & Paradoxornithidae & 褐鸦雀 & Paradoxornis unicolor & $\mathrm{hm}$ & \\
\hline 1233 & 鸟纲 & Aves & 雀形目 & Passeriformes & 鸦雀科 & Paradoxornithidae & 灰头鸦雀 & Paradoxornis gularis & $\mathrm{wc}$ & \\
\hline 1234 & 鸟纲 & Aves & 雀形目 & Passeriformes & 鸦雀科 & Paradoxornithidae & 三趾鸦雀 & Paradoxornis paradoxus & hc & \\
\hline 1235 & 鸟纲 & Aves & 雀形目 & Passeriformes & 鸦雀科 & Paradoxornithidae & 斑胸鸦雀 & Paradoxornis flavirostris & $\mathrm{sd}$ & \\
\hline 1236 & 鸟纲 & Aves & 雀形目 & Passeriformes & 鸦雀科 & Paradoxornithidae & 白眶鸦雀 & Paradoxornis conspicillatus & sn & \\
\hline 1237 & 鸟纲 & Aves & 雀形目 & Passeriformes & 鸦雀科 & Paradoxornithidae & 棕头鸦雀 & Paradoxornis webbianus & sv & \\
\hline 1238 & 鸟纲 & Aves & 雀形目 & Passeriformes & 鸦雀科 & Paradoxornithidae & 暗色鸦雀 & Paradoxornis zappeyi & o & \\
\hline 1239 & 鸟纲 & Aves & 雀形目 & Passeriformes & 鸦雀科 & Paradoxornithidae & 灰冠鸦雀 & Paradoxornis przewalskii & o & \\
\hline 1240 & 鸟纲 & Aves & 雀形目 & Passeriformes & 鸦雀科 & Paradoxornithidae & 黄额鸦雀 & Paradoxornis fulvifrons & $\mathrm{hm}$ & \\
\hline 1241 & 鸟纲 & Aves & 雀形目 & Passeriformes & 鸦雀科 & Paradoxornithidae & 黑喉鸦雀 & Paradoxornis nipalensis & $\mathrm{sd}$ & \\
\hline 1242 & 鸟纲 & Aves & 雀形目 & Passeriformes & 鸦雀科 & Paradoxornithidae & 短尾鸦雀 & Paradoxornis davidianus & o & \\
\hline 1243 & 鸟纲 & Aves & 雀形目 & Passeriformes & 鸦雀科 & Paradoxornithidae & 红头鸦雀 & Paradoxornis ruficeps & $\mathrm{hm}$ & \\
\hline 1244 & 鸟纲 & Aves & 雀形目 & Passeriformes & 鸦雀科 & Paradoxornithidae & 震旦鸦雀 & Paradoxornis heudei & $\mathrm{e}$ & \\
\hline 1245 & 鸟纲 & Aves & 雀形目 & Passeriformes & 扇尾莺科 & Cisticolidae & 棕扇尾莺 & Cisticola juncidis & 05 & \\
\hline 1246 & 鸟纲 & Aves & 雀形目 & Passeriformes & 扇尾莺科 & Cisticolidae & 金头扇尾莺 & Cisticola exilis & wc & \\
\hline 1247 & 鸟纲 & Aves & 雀形目 & Passeriformes & 扇尾莺科 & Cisticolidae & 山鹪莺 & PriMa crinigera & wa & \\
\hline 1248 & 鸟纲 & Aves & 雀形目 & Passeriformes & 扇尾莺科 & Cisticolidae & 褐山鹪鹋 & Prinia polychroa & wa & \\
\hline 1249 & 鸟纲 & Aves & 雀形目 & Passeriformes & 扇尾莺科 & Cisticolidae & 黑喉山鹪莺 & Prinia atrogularis & wb & \\
\hline 1250 & 鸟纲 & Aves & 雀形目 & Passeriformes & 扇尾莺科 & Cisticolidae & 暗冕山鹪莺 & Prinia rufescens & wb & \\
\hline 1251 & 鸟纲 & Aves & 雀形目 & Passeriformes & 扇尾莺科 & Cisticolidae & 灰胸山鹪莺 & Prinia hodgsonii & wc & \\
\hline 1252 & 鸟纲 & Aves & 雀形目 & Passeriformes & 扇尾莺科 & Cisticolidae & 黄腹山鹪莺 & Prinia flaviventris & wb & \\
\hline
\end{tabular}


郜二虎, 何杰坤, 王志臣, 徐扬, 唐小平, 江海声. 全国陆生野生动物调查单元区划方案. 生物多样性, 2017, 25 (12): 1321-1330.

http://www.biodiversity-science.net/CN/10.17520/biods.2017135

\begin{tabular}{|c|c|c|c|c|c|c|c|c|c|c|}
\hline $\begin{array}{r}\text { 序号 } \\
\text { No. }\end{array}$ & \multicolumn{2}{|c|}{$\begin{array}{c}\text { 纲 } \\
\text { Class }\end{array}$} & \multicolumn{2}{|c|}{$\begin{array}{c}\text { 目 } \\
\text { Order }\end{array}$} & \multicolumn{2}{|r|}{$\begin{array}{c}\text { 科 } \\
\text { Family }\end{array}$} & \multicolumn{2}{|r|}{$\begin{array}{l}\text { 物种名 } \\
\text { Species }\end{array}$} & \multirow{2}{*}{$\begin{array}{l}\begin{array}{c}\text { 分布型 } \\
\text { Chorotype }\end{array} \\
\text { wd }\end{array}$} & \multirow[t]{2}{*}{$\begin{array}{c}\text { 备注 } \\
\text { Remarks }\end{array}$} \\
\hline 1253 & 鸟纲 & Aves & 雀形目 & Passeriformes & 扇尾莺科 & Cisticolidae & 纯色山鹪蕮 & Prinia inornata & & \\
\hline 1254 & 鸟纲 & Aves & 雀形目 & Passeriformes & 莺科 & Sylviidae & 栗头地莺 & Tesia castaneocoronata & $\mathrm{hm}$ & \\
\hline 1255 & 鸟纲 & Aves & 雀形目 & Passeriformes & 莺科 & Sylviidae & 金冠地莺 & Tesia olivea & wc & \\
\hline 1256 & 鸟纲 & Aves & 雀形目 & Passeriformes & 莺科 & Sylviidae & 灰腹地譡 & Tesia cyaniventer & $\mathrm{wb}$ & \\
\hline 1257 & 鸟纲 & Aves & 雀形目 & Passeriformes & 莺科 & Sylviidae & 淡脚树莺 & Cettia pallidipes & wa & \\
\hline 1258 & 鸟纲 & Aves & 雀形目 & Passeriformes & 莺科 & Sylviidae & 强脚树蕅 & Cetfia fortipes & wd & \\
\hline 1259 & 鸟纲 & Aves & 雀形目 & Passeriformes & 莺科 & Sylviidae & 大树莺 & Cetfia major & $\mathrm{hm}$ & \\
\hline 1260 & 鸟纲 & Aves & 雀形目 & Passeriformes & 莺科 & Sylviidae & 异色树莺 & Cettia flavolivacea & $\mathrm{hm}$ & \\
\hline 1261 & 鸟纲 & Aves & 雀形目 & Passeriformes & 莺科 & Sylviidae & 黄腹树莺 & Cettia acanthizoides & $\mathrm{sd}$ & \\
\hline 1262 & 鸟纲 & Aves & 雀形目 & Passeriformes & 莺科 & Sylviidae & 棕顶树蒀 & Cettia brunnifrons & $\mathrm{hm}$ & \\
\hline 1263 & 鸟纲 & Aves & 雀形目 & Passeriformes & 莺科 & Sylviidae & 宽尾树荣 & Cettia cetti & 03 & \\
\hline 1264 & 鸟纲 & Aves & 雀形目 & Passeriformes & 莺科 & Sylviidae & 巨嘴短翅茑 & Bradypterus major & d & \\
\hline 1265 & 鸟纲 & Aves & 雀形目 & Passeriformes & 莺科 & Sylviidae & 高山短翅蒀 & Bradypterus mandelli & wc & \\
\hline 1266 & 鸟纲 & Aves & 雀形目 & Passeriformes & 莺科 & Sylviidae & 棕褐短翅莺 & Bradypterus luteoventris & sd & \\
\hline 1267 & 鸟纲 & Aves & 雀形目 & Passeriformes & 莺科 & Sylviidae & 黑斑蝗莺 & Locustella naevia & o3 & \\
\hline 1268 & 鸟纲 & Aves & 雀形目 & Passeriformes & 莺科 & Sylviidae & 小蝗譡 & Locustella certhiola & $\mathrm{m}$ & \\
\hline 1269 & 鸟纲 & Aves & 雀形目 & Passeriformes & 荣科 & Sylviidae & 蒲苇荣 & Acrocephalus schoenobaenus & 03 & \\
\hline 1270 & 鸟纲 & Aves & 雀形目 & Passeriformes & 莺科 & Sylviidae & 稻田苇莺 & Acrocephalus agricola & 03 & \\
\hline 1271 & 鸟纲 & Aves & 雀形目 & Passeriformes & 莺科 & Sylviidae & 大苇莺 & Acrocephalus arundinaceus & 05 & \\
\hline 1272 & 鸟纲 & Aves & 雀形目 & Passeriformes & 荣科 & Sylviidae & 靴篱窎 & Hippolais caligata & d & \\
\hline 1273 & 鸟纲 & Aves & 雀形目 & Passeriformes & 莺科 & Sylviidae & 栗头缝叶莺 & Orthotomus cucullatus & wb & \\
\hline 1274 & 鸟纲 & Aves & 雀形目 & Passeriformes & 莺科 & Sylviidae & 长尾缝叶窎 & Orthotomus sutorius & $\mathrm{wb}$ & \\
\hline
\end{tabular}


郜二虎, 何杰坤, 王志臣, 徐扬, 唐小平, 江海声. 全国陆生野生动物调查单元区划方案. 生物多样性, 2017, 25 (12): 1321-1330.

http://www.biodiversity-science.net/CN/10.17520/biods.2017135

\begin{tabular}{|c|c|c|c|c|c|c|c|c|c|c|}
\hline $\begin{array}{r}\text { 序号 } \\
\text { No. }\end{array}$ & \multicolumn{2}{|c|}{$\begin{array}{c}\text { 纲 } \\
\text { Class }\end{array}$} & \multicolumn{2}{|c|}{$\begin{array}{c}\text { 目 } \\
\text { Order }\end{array}$} & \multicolumn{2}{|r|}{$\begin{array}{c}\text { 科 } \\
\text { Family }\end{array}$} & \multicolumn{2}{|r|}{$\begin{array}{l}\text { 物种名 } \\
\text { Species }\end{array}$} & \multirow{2}{*}{$\begin{array}{l}\begin{array}{c}\text { 分布型 } \\
\text { Chorotype }\end{array} \\
\text { wb }\end{array}$} & \multirow[t]{2}{*}{$\begin{array}{c}\text { 备注 } \\
\text { Remarks }\end{array}$} \\
\hline 1275 & 鸟纲 & Aves & 雀形目 & Passeriformes & 莺科 & Sylviidae & 黑喉缝叶莺 & Orthotomus atrogularis & & \\
\hline 1276 & 鸟纲 & Aves & 雀形目 & Passeriformes & 莺科 & Sylviidae & 花彩雀莺 & Leptopoecile sophiae & pa & \\
\hline 1277 & 鸟纲 & Aves & 雀形目 & Passeriformes & 莺科 & Sylviidae & 凤头雀莺 & Leptopoecile elegans & hc & \\
\hline 1278 & 鸟纲 & Aves & 雀形目 & Passeriformes & 莺科 & Sylviidae & 吹喳柳蒀 & Phylloscopus collybita & u & \\
\hline 1279 & 鸟纲 & Aves & 雀形目 & Passeriformes & 莺科 & Sylviidae & 褐柳蒀 & Phylloscopus fuscatus & $\mathrm{m}$ & \\
\hline 1280 & 鸟纲 & Aves & 雀形目 & Passeriformes & 莺科 & Sylviidae & 黄腹柳蒀 & Phylloscopus affinis & $\mathrm{hm}$ & \\
\hline 1281 & 鸟纲 & Aves & 雀形目 & Passeriformes & 莺科 & Sylviidae & 棕腹柳荣 & Phylloscopus subaffinis & sv & \\
\hline 1282 & 鸟纲 & Aves & 雀形目 & Passeriformes & 莺科 & Sylviidae & 灰柳蒀 & Phylloscopus griseolus & pa & \\
\hline 1283 & 鸟纲 & Aves & 雀形目 & Passeriformes & 莺科 & Sylviidae & 巨嘴柳莺 & Phylloscopus schwarzi & $\mathrm{m}$ & \\
\hline 1284 & 鸟纲 & Aves & 雀形目 & Passeriformes & 莺科 & Sylviidae & 橙斑翅柳蒀 & Phylloscopus pulcher & $\mathrm{hm}$ & \\
\hline 1285 & 鸟纲 & Aves & 雀形目 & Passeriformes & 莺科 & Sylviidae & 黄腰柳莺 & Phylloscopus proregulus & $\mathrm{u}$ & \\
\hline 1286 & 鸟纲 & Aves & 雀形目 & Passeriformes & 莺科 & Sylviidae & 黄眉柳莺 & Phylloscopus inornatus & $\mathrm{u}$ & \\
\hline 1287 & 鸟纲 & Aves & 雀形目 & Passeriformes & 莺科 & Sylviidae & 极北柳莺 & Phylloscopus borealis & uc & \\
\hline 1288 & 鸟纲 & Aves & 雀形目 & Passeriformes & 莺科 & Sylviidae & 暗绿柳莺 & Phylloscopus trochiloides & $\mathrm{u}$ & \\
\hline 1289 & 鸟纲 & Aves & 雀形目 & Passeriformes & 莺科 & Sylviidae & 海南柳莺 & Phylloscopus hainanus & $\mathrm{j}$ & \\
\hline 1290 & 鸟纲 & Aves & 雀形目 & Passeriformes & 莺科 & Sylviidae & 白斑尾柳莺 & Phylloscopus davisoni & $\mathrm{sc}$ & \\
\hline 1291 & 鸟纲 & Aves & 雀形目 & Passeriformes & 荣科 & Sylviidae & 金眀鹟莺 & Seicercus burkii & $\mathrm{sd}$ & \\
\hline 1292 & 鸟纲 & Aves & 雀形目 & Passeriformes & 莺科 & Sylviidae & 灰头鹟莺 & Seicercus xanthoschistos & ha & \\
\hline 1293 & 鸟纲 & Aves & 雀形目 & Passeriformes & 莺科 & Sylviidae & 灰脸鹟莺 & Seicercus poliogenys & $\mathrm{hm}$ & \\
\hline 1294 & 鸟纲 & Aves & 雀形目 & Passeriformes & 莺科 & Sylviidae & 栗头鹟莺 & Seicercus castaniceps & wd & \\
\hline 1295 & 鸟纲 & Aves & 雀形目 & Passeriformes & 莺科 & Sylviidae & 棕脸蓊莺 & Abroscopus albogularis & sd & \\
\hline 1296 & 鸟纲 & Aves & 雀形目 & Passeriformes & 莺科 & Sylviidae & 黄腹䳜莺 & Abroscopus superciliaris & wa & \\
\hline
\end{tabular}


郜二虎, 何杰坤, 王志臣, 徐扬, 唐小平, 江海声. 全国陆生野生动物调查单元区划方案. 生物多样性, 2017, 25 (12): 1321-1330.

http://www.biodiversity-science.net/CN/10.17520/biods.2017135

\begin{tabular}{|c|c|c|c|c|c|c|c|c|c|c|}
\hline \multirow{2}{*}{$\begin{array}{l}\text { 序号 } \\
\text { No. } \\
1297\end{array}$} & \multicolumn{2}{|c|}{$\begin{array}{c}\text { 纲 } \\
\text { Class }\end{array}$} & \multicolumn{2}{|c|}{$\begin{array}{c}\text { 目 } \\
\text { Order }\end{array}$} & \multirow{2}{*}{\multicolumn{2}{|c|}{$\begin{array}{c}\text { 科 } \\
\text { Family } \\
\text { Sylviidae }\end{array}$}} & \multicolumn{2}{|r|}{$\begin{array}{l}\text { 物种名 } \\
\text { Species }\end{array}$} & \multirow{2}{*}{$\begin{array}{l}\begin{array}{c}\text { 分布型 } \\
\text { Chorotype }\end{array} \\
\text { wa }\end{array}$} & \multirow[t]{2}{*}{$\begin{array}{c}\text { 备注 } \\
\text { Remarks }\end{array}$} \\
\hline & 鸟纲 & Aves & 雀形目 & Passeriformes & & & 黑脸暡莺 & Abroscopus schisticeps & & \\
\hline 1298 & 鸟纲 & Aves & 雀形目 & Passeriformes & 莺科 & Sylviidae & 宽嘴除莺 & Tickellia hodgsoni & $\mathrm{hm}$ & \\
\hline 1299 & 鸟纲 & Aves & 雀形目 & Passeriformes & 莺科 & Sylviidae & 沼泽大尾莺 & Megalurus palustris & $\mathrm{wb}$ & \\
\hline 1300 & 鸟纲 & Aves & 雀形目 & Passeriformes & 莺科 & Sylviidae & 大草莺 & Graminicola bengalensis & wa & \\
\hline 1301 & 鸟纲 & Aves & 雀形目 & Passeriformes & 莺科 & Sylviidae & 白喉林譡 & Sylvia curruca & 03 & \\
\hline 1302 & 鸟纲 & Aves & 雀形目 & Passeriformes & 莺科 & Sylviidae & 漠白喉林蒀 & Sylvia minula & o3 & \\
\hline 1303 & 鸟纲 & Aves & 雀形目 & Passeriformes & 荣科 & Sylviidae & 横斑林莺 & Sylvia nisoria & 03 & \\
\hline 1304 & 鸟纲 & Aves & 雀形目 & Passeriformes & 戴菊科 & Regulidae & 戴菊 & Regulus regulus & $\mathrm{cf}$ & \\
\hline 1305 & 鸟纲 & Aves & 雀形目 & Passeriformes & 绣眼鸟科 & Zosteropidae & 灰腹绣眼鸟 & Zosterops palpebrosus & wc & \\
\hline 1306 & 鸟纲 & Aves & 雀形目 & Passeriformes & 攀雀科 & Remizidae & 火冠雀 & Cephalopyrus flammiceps & $\mathrm{hm}$ & \\
\hline 1307 & 鸟纲 & Aves & 雀形目 & Passeriformes & 长尾山雀科 & Aegithalidae & 银喉长尾山雀 & Aegithalos caudatus & $\mathrm{ub}$ & \\
\hline 1308 & 鸟纲 & Aves & 雀形目 & Passeriformes & 长尾山雀科 & Aegithalidae & 红头长尾山雀 & Aegithalos concinnus & wd & \\
\hline 1309 & 鸟纲 & Aves & 雀形目 & Passeriformes & 长尾山雀科 & Aegithalidae & 棕额长尾山雀 & Aegithalos iouschistos & $\mathrm{hm}$ & \\
\hline 1310 & 鸟纲 & Aves & 雀形目 & Passeriformes & 长尾山雀科 & Aegithalidae & 银脸长尾山雀 & Aegithalos fuliginosus & $\mathrm{pf}$ & \\
\hline 1311 & 鸟纲 & Aves & 雀形目 & Passeriformes & 山雀科 & Paridae & 沼泽山雀 & Parus palustris & $\mathrm{u}$ & \\
\hline 1312 & 鸟纲 & Aves & 雀形目 & Passeriformes & 山雀科 & Paridae & 褐头山雀 & Parus songarus & $\mathrm{cb}$ & \\
\hline 1313 & 鸟纲 & Aves & 雀形目 & Passeriformes & 山雀科 & Paridae & 白眉山雀 & Parus superciliosus & $\mathrm{pc}$ & \\
\hline 1314 & 鸟纲 & Aves & 雀形目 & Passeriformes & 山雀科 & Paridae & 红腹山雀 & Parus davidi & $\mathrm{pf}$ & \\
\hline 1315 & 鸟纲 & Aves & 雀形目 & Passeriformes & 山雀科 & Paridae & 煤山雀 & Parus ater & uf & \\
\hline 1316 & 鸟纲 & Aves & 雀形目 & Passeriformes & 山雀科 & Paridae & 黑冠山雀 & Parus rubidiventris & $\mathrm{hm}$ & \\
\hline 1317 & 鸟纲 & Aves & 雀形目 & Passeriformes & 山雀科 & Paridae & 黄腹山雀 & Parus venustulus & sh & \\
\hline 1318 & 鸟纲 & Aves & 雀形目 & Passeriformes & 山雀科 & Paridae & 褐冠山雀 & Parus dichrous & $\mathrm{hm}$ & \\
\hline
\end{tabular}


郜二虎, 何杰坤, 王志臣, 徐扬, 唐小平, 江海声. 全国陆生野生动物调查单元区划方案. 生物多样性, 2017, 25 (12): 1321-1330.

http://www.biodiversity-science.net/CN/10.17520/biods.2017135

\begin{tabular}{|c|c|c|c|c|c|c|c|c|c|c|}
\hline \multirow{2}{*}{$\begin{array}{c}\text { 序号 } \\
\text { No. } \\
1319\end{array}$} & \multicolumn{2}{|c|}{$\begin{array}{c}\text { 纲 } \\
\text { Class }\end{array}$} & \multicolumn{2}{|c|}{$\begin{array}{c}\text { 目 } \\
\text { Order }\end{array}$} & \multirow{2}{*}{\multicolumn{2}{|c|}{$\begin{array}{c}\text { 科 } \\
\text { Family } \\
\text { Paridae }\end{array}$}} & \multicolumn{2}{|r|}{$\begin{array}{l}\text { 物种名 } \\
\text { Species }\end{array}$} & \multirow{2}{*}{$\begin{array}{l}\begin{array}{c}\text { 分布型 } \\
\text { Chorotype }\end{array} \\
\text { o }\end{array}$} & \multirow[t]{2}{*}{$\begin{array}{c}\text { 备注 } \\
\text { Remarks }\end{array}$} \\
\hline & 鸟纲 & Aves & 雀形目 & Passeriformes & & & 大山雀 & Parus major & & \\
\hline 1320 & 鸟纲 & Aves & 雀形目 & Passeriformes & 山雀科 & Paridae & 西域山雀 & Parus bokharensis & d & \\
\hline 1321 & 鸟纲 & Aves & 雀形目 & Passeriformes & 山雀科 & Paridae & 绿背山雀 & Parus monticolus & wd & \\
\hline 1322 & 鸟纲 & Aves & 雀形目 & Passeriformes & 山雀科 & Paridae & 黄规山雀 & Parus spilonotus & wc & \\
\hline 1323 & 鸟纲 & Aves & 雀形目 & Passeriformes & 山雀科 & Paridae & 灰蓝山雀 & Parus cyanus & ue & \\
\hline 1324 & 鸟纲 & Aves & 雀形目 & Passeriformes & 山雀科 & Paridae & 杂色山雀 & Parus varius & $\mathrm{mc}$ & \\
\hline 1325 & 鸟纲 & Aves & 雀形目 & Passeriformes & 山雀科 & Paridae & 地山雀 & Pseudopodoces humilis & pa & \\
\hline 1326 & 鸟纲 & Aves & 雀形目 & Passeriformes & 山雀科 & Paridae & 黄眉林雀 & Sylviparus modestus & wd & \\
\hline 1327 & 鸟纲 & Aves & 雀形目 & Passeriformes & 山雀科 & Paridae & 冕雀 & Melanochlora sultanea & wb & \\
\hline 1328 & 鸟纲 & Aves & 雀形目 & Passeriformes & 鴄科 & Sittidae & 栗腹鴄 & Sitta castanea & wa & \\
\hline 1329 & 鸟纲 & Aves & 雀形目 & Passeriformes & 鳲科 & Sittidae & 普通鴄 & Sitta europaea & ub & \\
\hline 1330 & 鸟纲 & Aves & 雀形目 & Passeriformes & 鳲科 & Sittidae & 白尾鴄 & Sitta himalayensis & $\mathrm{hm}$ & \\
\hline 1331 & 鸟纲 & Aves & 雀形目 & Passeriformes & 鴄科 & Sittidae & 黑头鴄 & Sitta villosa & $\mathrm{cf}$ & \\
\hline 1332 & 鸟纲 & Aves & 雀形目 & Passeriformes & 鴄科 & Sittidae & 滇鴄 & Sitta yunnanensis & hc & \\
\hline 1333 & 鸟纲 & Aves & 雀形目 & Passeriformes & 鴄科 & Sittidae & 白脸鴄 & Sitta leucopsis & $\mathrm{hm}$ & \\
\hline 1334 & 鸟纲 & Aves & 雀形目 & Passeriformes & 鴄科 & Sittidae & 线额鴄 & Sitta frontalis & wc & \\
\hline 1335 & 鸟纲 & Aves & 雀形目 & Passeriformes & 鴄科 & Sittidae & 巨鳲 & Sitta magna & wb & \\
\hline 1336 & 鸟纲 & Aves & 雀形目 & Passeriformes & 鴄科 & Sittidae & 丽鴄 & Sitta formosa & $\mathrm{hm}$ & \\
\hline 1337 & 鸟纲 & Aves & 雀形目 & Passeriformes & 旋壁雀科 & Tichidromidae & 红翅旋壁雀 & Tichodroma muraria & o & \\
\hline 1338 & 鸟纲 & Aves & 雀形目 & Passeriformes & 旋木雀科 & Certhiidae & 欧亚旋木雀 & Certhia familiaris & $\mathrm{cb}$ & \\
\hline 1339 & 鸟纲 & Aves & 雀形目 & Passeriformes & 旋木雀科 & Certhiidae & 高山旋木雀 & Certhia himalayana & $\mathrm{hm}$ & \\
\hline 1340 & 鸟纲 & Aves & 雀形目 & Passeriformes & 旋木雀科 & Certhiidae & 红腹旋木雀 & Certhia nipalensis & ha & \\
\hline
\end{tabular}


郜二虎, 何杰坤, 王志臣, 徐扬, 唐小平, 江海声. 全国陆生野生动物调查单元区划方案. 生物多样性, 2017, 25 (12): 1321-1330.

http://www.biodiversity-science.net/CN/10.17520/biods.2017135

\begin{tabular}{|c|c|c|c|c|c|c|c|c|c|c|}
\hline $\begin{array}{r}\text { 序号 } \\
\text { No. }\end{array}$ & \multicolumn{2}{|c|}{$\begin{array}{c}\text { 纲 } \\
\text { Class }\end{array}$} & \multicolumn{2}{|c|}{$\begin{array}{c}\text { 目 } \\
\text { Order }\end{array}$} & \multicolumn{2}{|r|}{$\begin{array}{c}\text { 科 } \\
\text { Family }\end{array}$} & \multicolumn{2}{|r|}{$\begin{array}{l}\text { 物种名 } \\
\text { Species }\end{array}$} & \multirow{2}{*}{$\begin{array}{l}\begin{array}{c}\text { 分布型 } \\
\text { Chorotype }\end{array} \\
\mathrm{hm}\end{array}$} & \multirow[t]{2}{*}{$\begin{array}{c}\text { 备注 } \\
\text { Remarks }\end{array}$} \\
\hline 1341 & 鸟纲 & Aves & 雀形目 & Passeriformes & 旋木雀科 & Certhiidae & 褐喉旋木雀 & Certhia discolor & & \\
\hline 1342 & 鸟纲 & Aves & 雀形目 & Passeriformes & 啄花鸟科 & Dicaeidae & 厚嘴豚花鸟 & Dicaeum agile & wa & \\
\hline 1343 & 鸟纲 & Aves & 雀形目 & Passeriformes & 啄花鸟科 & Dicaeidae & 黄腹豚花鸟 & Dicaeum melanoxanthum & $\mathrm{hm}$ & \\
\hline 1344 & 鸟纲 & Aves & 雀形目 & Passeriformes & 啄花鸟科 & Dicaeidae & 纯色豚花鸟 & Dicaeum concolor & wd & \\
\hline 1345 & 鸟纲 & Aves & 雀形目 & Passeriformes & 啄花鸟科 & Dicaeidae & 红胸豚花鸟 & Dicaeum ignipectus & wd & \\
\hline 1346 & 鸟纲 & Aves & 雀形目 & Passeriformes & 啄花鸟科 & Dicaeidae & 朱背啄花鸟 & Dicaeum cruentatum & wb & \\
\hline 1347 & 鸟纲 & Aves & 雀形目 & Passeriformes & 花蜜鸟科 & Nectariniidae & 紫项太阳鸟 & Chalcoparia singalensis & wa & \\
\hline 1348 & 鸟纲 & Aves & 雀形目 & Passeriformes & 花蜜鸟科 & Nectariniidae & 蓝枕花蜜鸟 & Hypogramma hypogrammicum & wa & \\
\hline 1349 & 鸟纲 & Aves & 雀形目 & Passeriformes & 花蜜鸟科 & Nectariniidae & 紫花蜜鸟 & Cinnyris asiaticus & wa & \\
\hline 1350 & 鸟纲 & Aves & 雀形目 & Passeriformes & 花蜜鸟科 & Nectariniidae & 黄腹花蜜鸟 & Cinnyris jugularis & wa & \\
\hline 1351 & 鸟纲 & Aves & 雀形目 & Passeriformes & 花蜜鸟科 & Nectariniidae & 蓝喉太阳鸟 & Aethopyga gouldiae & sd & \\
\hline 1352 & 鸟纲 & Aves & 雀形目 & Passeriformes & 花蜜鸟科 & Nectariniidae & 绿喉太阳鸟 & Aethopyga nipalensis & $\mathrm{hm}$ & \\
\hline 1353 & 鸟纲 & Aves & 雀形目 & Passeriformes & 花蜜鸟科 & Nectariniidae & 叉尾太阳鸟 & Aethopyga christinae & $\mathrm{sc}$ & \\
\hline 1354 & 鸟纲 & Aves & 雀形目 & Passeriformes & 花蜜鸟科 & Nectariniidae & 黑胸太阳鸟 & Aethopyga saturata & wa & \\
\hline 1355 & 鸟纲 & Aves & 雀形目 & Passeriformes & 花蜜鸟科 & Nectariniidae & 黄腰太阳鸟 & Aethopyga siparaja & wa & \\
\hline 1356 & 鸟纲 & Aves & 雀形目 & Passeriformes & 花蜜鸟科 & Nectariniidae & 火尾太阳鸟 & Aethopyga ignicauda & ha & \\
\hline 1357 & 鸟纲 & Aves & 雀形目 & Passeriformes & 花蜜鸟科 & Nectariniidae & 长嘴捕蛛鸟 & Arachnothera longirostra & wa & \\
\hline 1358 & 鸟纲 & Aves & 雀形目 & Passeriformes & 花蜜鸟科 & Nectariniidae & 纹背捕蛛鸟 & Arachnothera magna & $\mathrm{hm}$ & \\
\hline 1359 & 鸟纲 & Aves & 雀形目 & Passeriformes & 雀科 & Passeridae & 黑顶麻雀 & Passer ammodendri & da & \\
\hline 1360 & 鸟纲 & Aves & 雀形目 & Passeriformes & 雀科 & Passeridae & 家麻雀 & Passer domesticus & $\mathrm{o} 1$ & \\
\hline 1361 & 鸟纲 & Aves & 雀形目 & Passeriformes & 雀科 & Passeridae & 黑胸麻雀 & Passer hispaniolensis & 03 & \\
\hline 1362 & 鸟纲 & Aves & 雀形目 & Passeriformes & 雀科 & Passeridae & 山麻雀 & Passer rutilans & sh & \\
\hline
\end{tabular}


郜二虎, 何杰坤, 王志臣, 徐扬, 唐小平, 江海声. 全国陆生野生动物调查单元区划方案. 生物多样性, 2017, 25 (12): 1321-1330.

http://www.biodiversity-science.net/CN/10.17520/biods.2017135

\begin{tabular}{|c|c|c|c|c|c|c|c|c|c|c|}
\hline \multirow{2}{*}{$\begin{array}{c}\text { 序号 } \\
\text { No. } \\
1363\end{array}$} & \multicolumn{2}{|c|}{$\begin{array}{c}\text { 纲 } \\
\text { Class }\end{array}$} & \multicolumn{2}{|c|}{$\begin{array}{c}\text { 目 } \\
\text { Order }\end{array}$} & \multicolumn{2}{|r|}{$\begin{array}{c}\text { 科 } \\
\text { Family }\end{array}$} & \multicolumn{2}{|r|}{$\begin{array}{l}\text { 物种名 } \\
\text { Species }\end{array}$} & \multirow{2}{*}{$\begin{array}{l}\text { 分布型 } \\
\text { Chorotype } \\
\text { uh }\end{array}$} & \multirow[t]{2}{*}{$\begin{array}{c}\text { 备注 } \\
\text { Remarks }\end{array}$} \\
\hline & 鸟纲 & Aves & 雀形目 & Passeriformes & 雀科 & Passeridae & 麻雀 & Passer montanus & & \\
\hline 1364 & 鸟纲 & Aves & 雀形目 & Passeriformes & 雀科 & Passeridae & 石雀 & Petronia petronia & o3 & \\
\hline 1365 & 鸟纲 & Aves & 雀形目 & Passeriformes & 雀科 & Passeridae & 白斑翅雪雀 & Montifringilla nivalis & pw & \\
\hline 1366 & 鸟纲 & Aves & 雀形目 & Passeriformes & 雀科 & Passeridae & 褐翅雪雀 & Montifringilla adamsi & py & \\
\hline 1367 & 鸟纲 & Aves & 雀形目 & Passeriformes & 雀科 & Passeridae & 白腰雪雀 & Onychostruthus taczanowskii & py & \\
\hline 1368 & 鸟纲 & Aves & 雀形目 & Passeriformes & 雀科 & Passeridae & 黑喉雪雀 & Pyrgilauda davidiana & $\mathrm{p}$ & \\
\hline 1369 & 鸟纲 & Aves & 雀形目 & Passeriformes & 雀科 & Passeridae & 棕颈雪雀 & Pyrgilauda ruficollis & py & \\
\hline 1370 & 鸟纲 & Aves & 雀形目 & Passeriformes & 雀科 & Passeridae & 棕背雪雀 & Pyrgilauda blanfordi & py & \\
\hline 1371 & 鸟纲 & Aves & 雀形目 & Passeriformes & 织雀科 & Ploceidae & 黄胸织雀 & Ploceus philippinus & wa & \\
\hline 1372 & 鸟纲 & Aves & 雀形目 & Passeriformes & 梅花雀科 & Estrildidae & 红梅花雀 & Amandava amandava & wa & \\
\hline 1373 & 鸟纲 & Aves & 雀形目 & Passeriformes & 梅花雀科 & Estrildidae & 白腰文鸟 & Lonchura striata & wd & \\
\hline 1374 & 鸟纲 & Aves & 雀形目 & Passeriformes & 梅花雀科 & Estrildidae & 斑文鸟 & Lonchura punctulata & wc & \\
\hline 1375 & 鸟纲 & Aves & 雀形目 & Passeriformes & 梅花雀科 & Estrildidae & 栗腹文鸟 & Lonchura malacca & wa & \\
\hline 1376 & 鸟纲 & Aves & 雀形目 & Passeriformes & 梅花雀科 & Estrildidae & 禾雀 & Padda oryzivora & $\mathrm{wb}$ & \\
\hline 1377 & 鸟纲 & Aves & 雀形目 & Passeriformes & 燕雀科 & Fringillidae & 燕雀 & Fringilla montifringilla & uc & \\
\hline 1378 & 鸟纲 & Aves & 雀形目 & Passeriformes & 燕雀科 & Fringillidae & 林岭雀 & Leucosticte nemoricola & pw & \\
\hline 1379 & 鸟纲 & Aves & 雀形目 & Passeriformes & 燕雀科 & Fringillidae & 高山岭雀 & Leucosticte brandti & pw & \\
\hline 1380 & 鸟纲 & Aves & 雀形目 & Passeriformes & 燕雀科 & Fringillidae & 粉红腹岭雀 & Leucosticte arctoa & $\mathrm{cc}$ & \\
\hline 1381 & 鸟纲 & Aves & 雀形目 & Passeriformes & 燕雀科 & Fringillidae & 红眉松雀 & Pinicola subhimachala & $\mathrm{hm}$ & \\
\hline 1382 & 鸟纲 & Aves & 雀形目 & Passeriformes & 燕雀科 & Fringillidae & 赤朱雀 & Carpodacus rubescens & $\mathrm{hm}$ & \\
\hline 1383 & 鸟纲 & Aves & 雀形目 & Passeriformes & 燕雀科 & Fringillidae & 暗胸朱雀 & Carpodacus nipalensis & $\mathrm{hm}$ & \\
\hline 1384 & 鸟纲 & Aves & 雀形目 & Passeriformes & 燕雀科 & Fringillidae & 普通朱雀 & Carpodacus erythrinus & $\mathrm{u}$ & \\
\hline
\end{tabular}


郜二虎, 何杰坤, 王志臣, 徐扬, 唐小平, 江海声. 全国陆生野生动物调查单元区划方案. 生物多样性, 2017, 25 (12): 1321-1330.

http://www.biodiversity-science.net/CN/10.17520/biods.2017135

\begin{tabular}{|c|c|c|c|c|c|c|c|c|c|c|}
\hline $\begin{array}{r}\text { 序号 } \\
\text { No. }\end{array}$ & \multicolumn{2}{|c|}{$\begin{array}{c}\text { 纲 } \\
\text { Class }\end{array}$} & \multicolumn{2}{|c|}{$\begin{array}{c}\text { 目 } \\
\text { Order }\end{array}$} & \multicolumn{2}{|r|}{$\begin{array}{c}\text { 科 } \\
\text { Family }\end{array}$} & \multicolumn{2}{|r|}{$\begin{array}{l}\text { 物种名 } \\
\text { Species }\end{array}$} & \multirow{2}{*}{$\begin{array}{l}\begin{array}{c}\text { 分布型 } \\
\text { Chorotype }\end{array} \\
\mathrm{hm}\end{array}$} & \multirow[t]{2}{*}{$\begin{array}{c}\text { 备注 } \\
\text { Remarks }\end{array}$} \\
\hline 1385 & 鸟纲 & Aves & 雀形目 & Passeriformes & 燕雀科 & Fringillidae & 红眉朱雀 & Carpodacus pulcherrimus & & \\
\hline 1386 & 鸟纲 & Aves & 雀形目 & Passeriformes & 燕雀科 & Fringillidae & 曙红朱雀 & Carpodacus eos & hc & \\
\hline 1387 & 鸟纲 & Aves & 雀形目 & Passeriformes & 燕雀科 & Fringillidae & 酒红朱雀 & Carpodacus vinaceus & hc & \\
\hline 1388 & 鸟纲 & Aves & 雀形目 & Passeriformes & 燕雀科 & Fringillidae & 棕朱雀 & Carpodacus edwardsii & $\mathrm{hm}$ & \\
\hline 1389 & 鸟纲 & Aves & 雀形目 & Passeriformes & 燕雀科 & Fringillidae & 沙色朱雀 & Carpodacus synoicus & $\mathrm{d}$ & \\
\hline 1390 & 鸟纲 & Aves & 雀形目 & Passeriformes & 燕雀科 & Fringillidae & 北朱雀 & Ckrpodacus roseus & $\mathrm{m}$ & \\
\hline 1391 & 鸟纲 & Aves & 雀形目 & Passeriformes & 燕雀科 & Fringillidae & 斑翅朱雀 & Carpodacus trifasciatus & he & \\
\hline 1392 & 鸟纲 & Aves & 雀形目 & Passeriformes & 燕雀科 & Fringillidae & 点翅朱雀 & Carpodacus rodopeplus & $\mathrm{hm}$ & \\
\hline 1393 & 鸟纲 & Aves & 雀形目 & Passeriformes & 燕雀科 & Fringillidae & 白眉朱雀 & Carpodacus thura & $\mathrm{hm}$ & \\
\hline 1394 & 鸟纲 & Aves & 雀形目 & Passeriformes & 燕雀科 & Fringillidae & 红腰朱雀 & Carpodacus rhodochlamys & pa & \\
\hline 1395 & 鸟纲 & Aves & 雀形目 & Passeriformes & 燕雀科 & Fringillidae & 拟大朱雀 & Carpodacus rubicilloides & $\mathrm{pz}$ & \\
\hline 1396 & 鸟纲 & Aves & 雀形目 & Passeriformes & 燕雀科 & Fringillidae & 大朱雀 & Carpodacus rubicilla & pw & \\
\hline 1397 & 鸟纲 & Aves & 雀形目 & Passeriformes & 燕雀科 & Fringillidae & 红胸朱雀 & Carpodacus puniceus & pw & \\
\hline 1398 & 鸟纲 & Aves & 雀形目 & Passeriformes & 燕雀科 & Fringillidae & 藏雀 & Kozlowia roborowskii & $\mathrm{p}$ & \\
\hline 1399 & 鸟纲 & Aves & 雀形目 & Passeriformes & 燕雀科 & Fringillidae & 红交嘴雀 & Loxia curvirostra & $\mathrm{cf}$ & \\
\hline 1400 & 鸟纲 & Aves & 雀形目 & Passeriformes & 燕雀科 & Fringillidae & 高山金翅雀 & Carduelis spinoides & wa & \\
\hline 1401 & 鸟纲 & Aves & 雀形目 & Passeriformes & 燕雀科 & Fringillidae & 黑头金翅雀 & Carduelis ambigua & $\mathrm{hm}$ & \\
\hline 1402 & 鸟纲 & Aves & 雀形目 & Passeriformes & 燕雀科 & Fringillidae & 黄雀 & Carduelis spinus & u & \\
\hline 1403 & 鸟纲 & Aves & 雀形目 & Passeriformes & 燕雀科 & Fringillidae & 藏黄雀 & Carduelis thibetana & $\mathrm{hm}$ & \\
\hline 1404 & 鸟纲 & Aves & 雀形目 & Passeriformes & 燕雀科 & Fringillidae & 红额金翅雀 & Carduelis carduelis & o & \\
\hline 1405 & 鸟纲 & Aves & 雀形目 & Passeriformes & 燕雀科 & Fringillidae & 金翅雀 & Carduelis sinica & me & \\
\hline 1406 & 鸟纲 & Aves & 雀形目 & Passeriformes & 燕雀科 & Fringillidae & 黄嘴朱顶雀 & Carduelis flavirostris & $\mathrm{u}$ & \\
\hline
\end{tabular}


郜二虎, 何杰坤, 王志臣, 徐扬, 唐小平, 江海声. 全国陆生野生动物调查单元区划方案. 生物多样性, 2017, 25 (12): 1321-1330.

http://www.biodiversity-science.net/CN/10.17520/biods.2017135

\begin{tabular}{|c|c|c|c|c|c|c|c|c|c|c|}
\hline $\begin{array}{r}\text { 序号 } \\
\text { No. }\end{array}$ & \multicolumn{2}{|c|}{$\begin{array}{c}\text { 纲 } \\
\text { Class }\end{array}$} & \multicolumn{2}{|c|}{$\begin{array}{c}\text { 目 } \\
\text { Order }\end{array}$} & \multicolumn{2}{|r|}{$\begin{array}{c}\text { 科 } \\
\text { Family }\end{array}$} & \multicolumn{2}{|r|}{$\begin{array}{l}\text { 物种名 } \\
\text { Species }\end{array}$} & \multirow{2}{*}{$\begin{array}{l}\begin{array}{c}\text { 分布型 } \\
\text { Chorotype }\end{array} \\
03\end{array}$} & \multirow[t]{2}{*}{$\begin{array}{c}\text { 备注 } \\
\text { Remarks }\end{array}$} \\
\hline 1407 & 鸟纲 & Aves & 雀形目 & Passeriformes & 燕雀科 & Fringillidae & 赤胸朱顶雀 & Carduelis cannabina & & \\
\hline 1408 & 鸟纲 & Aves & 雀形目 & Passeriformes & 燕雀科 & Fringillidae & 金额丝雀 & Serinus pusillus & o & \\
\hline 1409 & 鸟纲 & Aves & 雀形目 & Passeriformes & 燕雀科 & Fringillidae & 褐灰雀 & Pyrrhula nipalensis & wb & \\
\hline 1410 & 鸟纲 & Aves & 雀形目 & Passeriformes & 燕雀科 & Fringillidae & 红头灰雀 & Pyrrhula erythrocephala & ha & \\
\hline 1411 & 鸟纲 & Aves & 雀形目 & Passeriformes & 燕雀科 & Fringillidae & 灰头灰雀 & Pyrrhula erythaca & $\mathrm{hm}$ & \\
\hline 1412 & 鸟纲 & Aves & 雀形目 & Passeriformes & 燕雀科 & Fringillidae & 灰腹灰雀 & Pyrrhula griseiventris & uc & \\
\hline 1413 & 鸟纲 & Aves & 雀形目 & Passeriformes & 燕雀科 & Fringillidae & 锡嘴雀 & Coccothraustes coccothraustes & uc & \\
\hline 1414 & 鸟纲 & Aves & 雀形目 & Passeriformes & 燕雀科 & Fringillidae & 黄颈拟蜡嘴雀 & Mycerobas affinis & $\mathrm{hm}$ & \\
\hline 1415 & 鸟纲 & Aves & 雀形目 & Passeriformes & 燕雀科 & Fringillidae & 白点翅拟蜡嘴雀 & Mycerobas melanozanthos & $\mathrm{hm}$ & \\
\hline 1416 & 鸟纲 & Aves & 雀形目 & Passeriformes & 燕雀科 & Fringillidae & 白斑翅拟蜡嘴雀 & Mycerobas carnipes & pw & \\
\hline 1417 & 鸟纲 & Aves & 雀形目 & Passeriformes & 燕雀科 & Fringillidae & 金枕黑雀 & Pyrrhoplectes epauletta & $\mathrm{hm}$ & \\
\hline 1418 & 鸟纲 & Aves & 雀形目 & Passeriformes & 燕雀科 & Fringillidae & 巨嘴沙雀 & Rhodopechys obsoleta & d & \\
\hline 1419 & 鸟纲 & Aves & 雀形目 & Passeriformes & 燕雀科 & Fringillidae & 长尾雀 & Uragus sibiricus & $\mathrm{m}$ & \\
\hline 1420 & 鸟纲 & Aves & 雀形目 & Passeriformes & 燕雀科 & Fringillidae & 血雀 & Haematospiza sipahi & $\mathrm{hm}$ & \\
\hline 1421 & 鸟纲 & Aves & 雀形目 & Passeriformes & 鹌科 & Emberizidae & 朱㱐鸟 & Urocynchramus pylzowi & $\mathrm{pf}$ & \\
\hline 1422 & 鸟纲 & Aves & 雀形目 & Passeriformes & 鹉科 & Emberizidae & 凤头社 & Melophus lathami & wc & \\
\hline 1423 & 鸟纲 & Aves & 雀形目 & Passeriformes & 鸫科 & Emberizidae & 蓝鴊 & Latoucheornis siemsseni & hc & \\
\hline 1424 & 鸟纲 & Aves & 雀形目 & Passeriformes & 鸫科 & Emberizidae & 白头鸥 & Emberiza leucocephalos & u & \\
\hline 1425 & 鸟纲 & Aves & 雀形目 & Passeriformes & 鹉科 & Emberizidae & 藏坐 & Emberiza koslowi & $\mathrm{p}$ & \\
\hline 1426 & 鸟纲 & Aves & 雀形目 & Passeriformes & 鹉科 & Emberizidae & 灰眉岩鸥 & Emberiza godlewskii & 03 & \\
\hline 1427 & 鸟纲 & Aves & 雀形目 & Passeriformes & 鸫科 & Emberizidae & 三道眉草鸥 & Emberiza cioides & $\mathrm{mg}$ & \\
\hline 1428 & 鸟纲 & Aves & 雀形目 & Passeriformes & 鹉科 & Emberizidae & 栗斑腹鸤 & Emberiza jankowskii & $\mathrm{m}$ & \\
\hline
\end{tabular}


郜二虎, 何杰坤, 王志臣, 徐扬, 唐小平, 江海声. 全国陆生野生动物调查单元区划方案. 生物多样性, 2017, 25 (12): 1321-1330.

http://www.biodiversity-science.net/CN/10.17520/biods.2017135

\begin{tabular}{|c|c|c|c|c|c|c|c|c|c|c|}
\hline $\begin{array}{r}\text { 序号 } \\
\text { No. }\end{array}$ & \multicolumn{2}{|c|}{$\begin{array}{c}\text { 纲 } \\
\text { Class }\end{array}$} & \multicolumn{2}{|c|}{$\begin{array}{c}\text { 目 } \\
\text { Order }\end{array}$} & \multicolumn{2}{|r|}{$\begin{array}{c}\text { 科 } \\
\text { Family }\end{array}$} & \multicolumn{2}{|r|}{$\begin{array}{l}\text { 物种名 } \\
\text { Species }\end{array}$} & \multirow{2}{*}{$\begin{array}{l}\begin{array}{c}\text { 分布型 } \\
\text { Chorotype }\end{array} \\
d\end{array}$} & \multirow[t]{2}{*}{$\begin{array}{c}\text { 备注 } \\
\text { Remarks }\end{array}$} \\
\hline 1429 & 鸟纲 & Aves & 雀形目 & Passeriformes & 鸫科 & Emberizidae & 灰颈鸥 & Emberiza buchanani & & \\
\hline 1430 & 鸟纲 & Aves & 雀形目 & Passeriformes & 鹉科 & Emberizidae & 戋躯鸟 & Emberiza hortulana & 03 & \\
\hline 1431 & 鸟纲 & Aves & 雀形目 & Passeriformes & 烃科 & Emberizidae & 黄胸巫 & Emberiza aureola & $\mathrm{ub}$ & \\
\hline 1432 & 鸟纲 & Aves & 雀形目 & Passeriformes & 鸫科 & Emberizidae & 褐头剢鸟 & Emberiza bruniceps & d & \\
\hline 1433 & 鸟纲 & Aves & 雀形目 & Passeriformes & 鸫科 & Emberizidae & 芦鸤 & Emberiza schoeniclus & ua & \\
\hline 1434 & 鸟纲 & Aves & 雀形目 & Passeriformes & 鸫科 & Emberizidae & 㯟坼 & Emberiza calandra & 03 & \\
\hline 1435 & 哺乳纲 & Mammalia & 食虫目 & Insectivora & 猬科 & Erinaceidae & 毛猬 & Hylomys suillus & wa & \\
\hline 1436 & 哺乳纲 & Mammalia & 食虫目 & Insectivora & 猬科 & Erinaceidae & 海南毛猬 & Neohylomys hainanensis & $\mathrm{j}$ & \\
\hline 1437 & 哺乳纲 & Mammalia & 食虫目 & Insectivora & 猬科 & Erinaceidae & 鼠猬 & Neotetracus sinensis & $\mathrm{sd}$ & \\
\hline 1438 & 哺乳纲 & Mammalia & 食虫目 & Insectivora & 猬科 & Erinaceidae & 大耳猬 & Hemiechinus auritus & $\mathrm{d}$ & \\
\hline 1439 & 哺乳纲 & Mammalia & 食虫目 & Insectivora & 猬科 & Erinaceidae & 达乌尔猬 & Hemiechinus dauuricus & $\mathrm{dn}$ & \\
\hline 1440 & 哺乳纲 & Mammalia & 食虫目 & Insectivora & 猬科 & Erinaceidae & 林猬 & Mesechinus hughi & o & \\
\hline 1441 & 哺乳纲 & Mammalia & 食虫目 & Insectivora & 鼠晏科 & Talpidae & 长吻鼠鼠 & Nasillus gracilis & sh & \\
\hline 1442 & 哺乳纲 & Mammalia & 食虫目 & Insectivora & 鼠科 & Talpidae & 峨眉鼣鼠 & Nasillus andesoni & hc & \\
\hline 1443 & 哺乳纲 & Mammalia & 食虫目 & Insectivora & 鼠科 & Talpidae & 鼠鼠 & Uropsilus soricipes & hc & \\
\hline 1444 & 哺乳纲 & Mammalia & 食虫目 & Insectivora & 鼠科 & Talpidae & 长尾鼣鼠 & Scaptonyx fusicaudus & hc & \\
\hline 1445 & 哺乳纲 & Mammalia & 食虫目 & Insectivora & 鼠科 & Talpidae & 甘肃鼠 & Scapanulus oweni & hc & \\
\hline 1446 & 哺乳纲 & Mammalia & 食虫目 & Insectivora & 鼠科 & Talpidae & 小缺齿鼠 & Mogera wogura & $\mathrm{kf}$ & \\
\hline 1447 & 哺乳纲 & Mammalia & 食虫目 & Insectivora & 鼠科 & Talpidae & 大缺齿鼠 & Mogera robusta & $\mathrm{kb}$ & \\
\hline 1448 & 哺乳纲 & Mammalia & 食虫目 & Insectivora & 鼠科 & Talpidae & 华南缺齿鼠 & Mogera insularis & $\mathrm{sc}$ & \\
\hline 1449 & 哺乳纲 & Mammalia & 食虫目 & Insectivora & 鼠科 & Talpidae & 白尾鼠 & Parascaptor leucurus & wb & \\
\hline 1450 & 哺乳纲 & Mammalia & 食虫目 & Insectivora & 鼠科 & Talpidae & 麻鼠 & Scaptochirus moschatus & ba & \\
\hline
\end{tabular}


郜二虎, 何杰坤, 王志臣, 徐扬, 唐小平, 江海声. 全国陆生野生动物调查单元区划方案. 生物多样性, 2017, 25 (12): 1321-1330.

http://www.biodiversity-science.net/CN/10.17520/biods.2017135

\begin{tabular}{|c|c|c|c|c|c|c|c|c|c|c|}
\hline \multirow{2}{*}{$\begin{array}{c}\text { 序号 } \\
\text { No. } \\
1451\end{array}$} & \multicolumn{2}{|c|}{$\begin{array}{c}\text { 纲 } \\
\text { Class }\end{array}$} & \multicolumn{2}{|c|}{$\begin{array}{c}\text { 目 } \\
\text { Order }\end{array}$} & \multirow{2}{*}{\multicolumn{2}{|c|}{$\begin{array}{c}\text { 科 } \\
\text { Family } \\
\text { Soricidae }\end{array}$}} & \multirow{2}{*}{\multicolumn{2}{|c|}{$\begin{array}{c}\begin{array}{c}\text { 物种名 } \\
\text { Species }\end{array} \\
\text { Sorex minutus }\end{array}$}} & \multirow{2}{*}{\begin{tabular}{|l}
\multicolumn{1}{c}{$\begin{array}{c}\text { 分布型 } \\
\text { Chorotype }\end{array}$} \\
ub
\end{tabular}} & \multirow[t]{2}{*}{$\begin{array}{c}\text { 备注 } \\
\text { Remarks }\end{array}$} \\
\hline & 哺乳纲 & Mammalia & 食虫目 & Insectivora & & & & & & \\
\hline 1452 & 哺乳纲 & Mammalia & 食虫目 & Insectivora & 鼠鼠青科 & Soricidae & 中舀鼣 & Sorex caecutiens & ue & \\
\hline 1453 & 哺乳纲 & Mammalia & 食虫目 & Insectivora & 鼠鼠青科 & Soricidae & 长爪鼠鼠 & Sorex unguiculatus & $\mathrm{mg}$ & \\
\hline 1454 & 哺乳纲 & Mammalia & 食虫目 & Insectivora & 鼠鼠青科 & Soricidae & 大齿鼣鼠 & Sorex daphaenodon & $\mathrm{m}$ & \\
\hline 1455 & 哺乳纲 & Mammalia & 食虫目 & Insectivora & 鼠鼠青科 & Soricidae & 大鼠鼠 & Sorex mirabilis & ke & \\
\hline 1456 & 哺乳纲 & Mammalia & 食虫目 & Insectivora & 鼠鼠青科 & Soricidae & 纹背鼩鼠 & Sorex cylindricauda & hc & \\
\hline 1457 & 哺乳纲 & Mammalia & 食虫目 & Insectivora & 鼠鼠青科 & Soricidae & 小纹背鼩鼣 & Sorex bedfordiae & hc & \\
\hline 1458 & 哺乳纲 & Mammalia & 食虫目 & Insectivora & 鼠鼣青科 & Soricidae & 黑齿舄鼠 & Blarinella quadraticauda & hc & \\
\hline 1459 & 哺乳纲 & Mammalia & 食虫目 & Insectivora & 鼠鼠青科 & Soricidae & 大爪长尾䳔鼱 & Soriculus nigrescens & ha & \\
\hline 1460 & 哺乳纲 & Mammalia & 食虫目 & Insectivora & 鼠鼠青科 & Soricidae & 褐腹长尾鼠鼣 & Soriculus caudatus & $\mathrm{hm}$ & \\
\hline 1461 & 哺乳纲 & Mammalia & 食虫目 & Insectivora & 鼠鼠青科 & Soricidae & 台湾长尾鼠鼱 & Soriculus fumidus & $\mathrm{j}$ & \\
\hline 1462 & 哺乳纲 & Mammalia & 食虫目 & Insectivora & 鼠鼣青科 & Soricidae & 大长尾㱍鼠 & Soriculus leucops & $\mathrm{hm}$ & \\
\hline 1463 & 哺乳纲 & Mammalia & 食虫目 & Insectivora & 鼠甸青科 & Soricidae & 川西缺齿鼠鼠 & Chodsigoa hypsibius & hc & \\
\hline 1464 & 哺乳纲 & Mammalia & 食虫目 & Insectivora & 鼠鼠青科 & Soricidae & 水鼠鼠 & Neomys fodiens & $\mathrm{ub}$ & \\
\hline 1465 & 哺乳纲 & Mammalia & 食虫目 & Insectivora & 鼠鼠青科 & Soricidae & 微尾鼣 & Anourosorex squamipes & sd & \\
\hline 1466 & 哺乳纲 & Mammalia & 食虫目 & Insectivora & 鼠鼣青科 & Soricidae & 喜马拉雅水舄 & Chimmarogale himalayicus & sv & \\
\hline 1467 & 哺乳纲 & Mammalia & 食虫目 & Insectivora & 鼠鼠青科 & Soricidae & 灰腹水鼠 & Chimmarogale styani & hc & \\
\hline 1468 & 哺乳纲 & Mammalia & 食虫目 & Insectivora & 鼠鼠青科 & Soricidae & 蹼足舄 & Nectogale elegans & hc & \\
\hline 1469 & 哺乳纲 & Mammalia & 食虫目 & Insectivora & 鼠鼠青科 & Soricidae & 大臭舀 & Suncus murinus & wd & \\
\hline 1470 & 哺乳纲 & Mammalia & 食虫目 & Insectivora & 鼠鼠青科 & Soricidae & 小臭鼣 & Suncus etruscus & wa & \\
\hline 1471 & 哺乳纲 & Mammalia & 食虫目 & Insectivora & 鼠鼠青科 & Soricidae & 南小榭鼣 & Crocidura horsfieldi & wd & \\
\hline 1472 & 哺乳纲 & Mammalia & 食虫目 & Insectivora & 鼠鼣科 & Soricidae & 灰榭鼩 & Crocidura attenuata & $\mathrm{sd}$ & \\
\hline
\end{tabular}


郜二虎, 何杰坤, 王志臣, 徐扬, 唐小平, 江海声. 全国陆生野生动物调查单元区划方案. 生物多样性, 2017, 25 (12): 1321-1330.

http://www.biodiversity-science.net/CN/10.17520/biods.2017135

\begin{tabular}{|c|c|c|c|c|c|c|c|c|c|c|}
\hline $\begin{array}{r}\text { 序号 } \\
\text { No. }\end{array}$ & \multicolumn{2}{|c|}{$\begin{array}{c}\text { 纲 } \\
\text { Class }\end{array}$} & \multicolumn{2}{|c|}{$\begin{array}{c}\text { 目 } \\
\text { Order }\end{array}$} & \multicolumn{2}{|r|}{$\begin{array}{c}\text { 科 } \\
\text { Family }\end{array}$} & \multicolumn{2}{|r|}{$\begin{array}{l}\text { 物种名 } \\
\text { Species }\end{array}$} & \multirow{2}{*}{$\begin{array}{l}\begin{array}{c}\text { 分布型 } \\
\text { Chorotype }\end{array} \\
\text { o }\end{array}$} & \multirow[t]{2}{*}{$\begin{array}{c}\text { 备注 } \\
\text { Remark }\end{array}$} \\
\hline 1473 & 哺乳纲 & Mammalia & 食虫目 & Insectivora & 鼠鼠科 & Soricidae & 大厡鼣 & Crocidura lasiura & & \\
\hline 1474 & 哺乳纲 & Mammalia & 攀舄回目 & Scandentia & 树鼠科 & Tupaiidae & 北树鼣 & Tupaia belangeri & wb & \\
\hline 1475 & 哺乳纲 & Mammalia & 灵长目 & Primates & 獭猴科 & Lorisidae & 蜂猴 & Nycticebus bengalensis & wa & \\
\hline 1476 & 哺乳纲 & Mammalia & 灵长目 & Primates & 懒猴科 & Lorisidae & 倭蜂猴 & Nycticebus pygmaeus & wa & \\
\hline 1477 & 哺乳纲 & Mammalia & 灵长目 & Primates & 猴科 & Cercopithecidae & 北豚尾猴 & Macaca leonina & wa & \\
\hline 1478 & 哺乳纲 & Mammalia & 灵长目 & Primates & 猴科 & Cercopithecidae & 猕猴 & Macaca mulatta & we & \\
\hline 1479 & 哺乳纲 & Mammalia & 灵长目 & Primates & 猴科 & Cercopithecidae & 熊猴 & Macaca assamensis & we & \\
\hline 1480 & 哺乳纲 & Mammalia & 灵长目 & Primates & 猴科 & Cercopithecidae & 台湾猴 & Macaca cyclopis & $\mathrm{j}$ & \\
\hline 1481 & 哺乳纲 & Mammalia & 灵长目 & Primates & 猴科 & Cercopithecidae & 短尾猴 & Macaca arctoides & $\mathrm{wb}$ & \\
\hline 1482 & 哺乳纲 & Mammalia & 灵长目 & Primates & 猴科 & Cercopithecidae & 藏酋猴 & Macaca thibetana & se & \\
\hline 1483 & 哺乳纲 & Mammalia & 灵长目 & Primates & 猴科 & Cercopithecidae & 戴帽叶猴 & Trachypithecus shortridgei & wa & \\
\hline 1484 & 哺乳纲 & Mammalia & 灵长目 & Primates & 猴科 & Cercopithecidae & 灰叶猴 & Trachypithecus phayrei & $\mathrm{wb}$ & \\
\hline 1485 & 哺乳纲 & Mammalia & 灵长目 & Primates & 猴科 & Cercopithecidae & 黑叶猴 & Trachypithecus francoisi & wc & \\
\hline 1486 & 哺乳纲 & Mammalia & 灵长目 & Primates & 猴科 & Cercopithecidae & 川金丝猴 & Rhinopithecus roxellana & hc & \\
\hline 1487 & 哺乳纲 & Mammalia & 灵长目 & Primates & 猴科 & Cercopithecidae & 滇金丝猴 & Rhinopithecus bieti & hc & \\
\hline 1488 & 哺乳纲 & Mammalia & 灵长目 & Primates & 猴科 & Cercopithecidae & 黔金丝猴 & Rhinopithecus brelichi & hc & \\
\hline 1489 & 哺乳纲 & Mammalia & 灵长目 & Primates & 长臂猿科 & Hylobatidae & 黑冠长臂猿 & Hylobates concolor & wb & 现分为 2 种 \\
\hline 1490 & 哺乳纲 & Mammalia & 灵长目 & Primates & 长臂猿科 & Hylobatidae & 海南长臂猿 & Hylobates hainanus & $\mathrm{j}$ & \\
\hline 1491 & 哺乳纲 & Mammalia & 灵长目 & Primates & 长臂猿科 & Hylobatidae & 白㚘长臂猿 & Hylobates leucogenys & wa & \\
\hline 1492 & 哺乳纲 & Mammalia & 灵长目 & Primates & 长臂猿科 & Hylobatidae & 白掌长臂猿 & Hylobates lar & wa & \\
\hline 1493 & 哺乳纲 & Mammalia & 灵长目 & Primates & 长臂猿科 & Hylobatidae & 白眉长臂猿 & Hylobates hoolock & wa & \\
\hline 1494 & 哺乳纲 & Mammalia & 鳞甲目 & Pholidota & 鲮鲤科 & Manidae & 中国穿山甲 & Manis pentadactyla & wc & \\
\hline
\end{tabular}


郜二虎, 何杰坤, 王志臣, 徐扬, 唐小平, 江海声. 全国陆生野生动物调查单元区划方案. 生物多样性, 2017, 25 (12): 1321-1330.

http://www.biodiversity-science.net/CN/10.17520/biods.2017135

\begin{tabular}{|c|c|c|c|c|c|c|c|c|c|c|}
\hline $\begin{array}{r}\text { 序号 } \\
\text { No. }\end{array}$ & \multicolumn{2}{|c|}{$\begin{array}{c}\text { 纲 } \\
\text { Class }\end{array}$} & \multicolumn{2}{|c|}{$\begin{array}{c}\text { 目 } \\
\text { Order }\end{array}$} & \multicolumn{2}{|r|}{$\begin{array}{c}\text { 科 } \\
\text { Family }\end{array}$} & \multicolumn{2}{|r|}{$\begin{array}{l}\text { 物种名 } \\
\text { Species }\end{array}$} & \multirow{2}{*}{$\begin{array}{l}\begin{array}{c}\text { 分布型 } \\
\text { Chorotype }\end{array} \\
\text { wa }\end{array}$} & \multirow[t]{2}{*}{$\begin{array}{c}\text { 备注 } \\
\text { Remarks }\end{array}$} \\
\hline 1495 & 哺乳纲 & Mammalia & 鳞甲目 & Pholidota & 鲮鲤科 & Manidae & 印度穿山甲 & Manis crassicaudata & & \\
\hline 1496 & 哺乳纲 & Mammalia & 食肉目 & Carnivora & 犬科 & Canidae & 狼 & Canis lupus & $\mathrm{ch}$ & \\
\hline 1497 & 哺乳纲 & Mammalia & 食肉目 & Carnivora & 犬科 & Canidae & 赤狐 & Vulpes vulpes & $\mathrm{ch}$ & \\
\hline 1498 & 哺乳纲 & Mammalia & 食肉目 & Carnivora & 犬科 & Canidae & 沙狐 & Vulpes corsac & $\mathrm{dk}$ & \\
\hline 1499 & 哺乳纲 & Mammalia & 食肉目 & Carnivora & 犬科 & Canidae & 藏沙狐 & Vulpes ferrilata & pa & \\
\hline 1500 & 哺乳纲 & Mammalia & 食肉目 & Carnivora & 犬科 & Canidae & 貉 & Nyctereutes procyonoides & eg & \\
\hline 1501 & 哺乳纲 & Mammalia & 食肉目 & Carnivora & 犬科 & Canidae & 豹 & Cuon alpinus & we & \\
\hline 1502 & 哺乳纲 & Mammalia & 食肉目 & Carnivora & 熊科 & Ursidae & 棕熊 & Ursus arctos & $\mathrm{ca}$ & \\
\hline 1503 & 哺乳纲 & Mammalia & 食肉目 & Carnivora & 熊科 & Ursidae & 黑熊 & Selenarctos thibetanus & eg & \\
\hline 1504 & 哺乳纲 & Mammalia & 食肉目 & Carnivora & 熊科 & Ursidae & 马来熊 & Helarctos malayanus & wa & \\
\hline 1505 & 哺乳纲 & Mammalia & 食肉目 & Carnivora & 大熊猫科 & Ailuropodidae & 大熊猫 & Ailuropoda melanoleuca & hc & \\
\hline 1506 & 哺乳纲 & Mammalia & 食肉目 & Carnivora & 小熊猫科 & Ailuridae & 小熊猫 & Ailurus fulgens & $\mathrm{hm}$ & \\
\hline 1507 & 哺乳纲 & Mammalia & 食肉目 & Carnivora & 鼠由科 & Mustelidae & 石貂 & Martes foina & $\mathrm{u}$ & \\
\hline 1508 & 哺乳纲 & Mammalia & 食肉目 & Carnivora & 鼠科 & Mustelidae & 紫貂 & Martes zibellina & uc & \\
\hline 1509 & 哺乳纲 & Mammalia & 食肉目 & Carnivora & 鼠由科 & Mustelidae & 青鼣 & Martes flavigula & we & \\
\hline 1510 & 哺乳纲 & Mammalia & 食肉目 & Carnivora & 鼠由科 & Mustelidae & 貂熊 & Gulo gulo & $\mathrm{cc}$ & \\
\hline 1511 & 哺乳纲 & Mammalia & 食肉目 & Carnivora & 鼠由科 & Mustelidae & 白鼣 & Mustela erminea & $\mathrm{cf}$ & \\
\hline 1512 & 哺乳纲 & Mammalia & 食肉目 & Carnivora & 鼠科 & Mustelidae & 伶䶅 & Mustela nivalis & uf & \\
\hline 1513 & 哺乳纲 & Mammalia & 食肉目 & Carnivora & 鼠由科 & Mustelidae & 香鼣 & Mustela altaica & o & \\
\hline 1514 & 哺乳纲 & Mammalia & 食肉目 & Carnivora & 鼠由科 & Mustelidae & 黄腹䶅 & Mustela kathiah & sd & \\
\hline 1515 & 哺乳纲 & Mammalia & 食肉目 & Carnivora & 鼠由科 & Mustelidae & 黄鼣 & Mustela sibirica & uh & \\
\hline 1516 & 哺乳纲 & Mammalia & 食肉目 & Carnivora & 鼠科 & Mustelidae & 纹鼬 & Mustela strigidorsa & wa & \\
\hline
\end{tabular}


郜二虎, 何杰坤, 王志臣, 徐扬, 唐小平, 江海声. 全国陆生野生动物调查单元区划方案. 生物多样性, 2017, 25 (12): 1321-1330.

http://www.biodiversity-science.net/CN/10.17520/biods.2017135

\begin{tabular}{|c|c|c|c|c|c|c|c|c|c|c|}
\hline $\begin{array}{r}\text { 序号 } \\
\text { No. }\end{array}$ & \multicolumn{2}{|c|}{$\begin{array}{c}\text { 纲 } \\
\text { Class }\end{array}$} & \multicolumn{2}{|c|}{$\begin{array}{c}\text { 目 } \\
\text { Order }\end{array}$} & \multicolumn{2}{|r|}{$\begin{array}{c}\text { 科 } \\
\text { Family }\end{array}$} & \multicolumn{2}{|r|}{$\begin{array}{l}\text { 物种名 } \\
\text { Species }\end{array}$} & \multirow{2}{*}{$\begin{array}{l}\begin{array}{c}\text { 分布型 } \\
\text { Chorotype }\end{array} \\
\text { uf }\end{array}$} & \multirow[t]{2}{*}{$\begin{array}{c}\text { 备注 } \\
\text { Remarks }\end{array}$} \\
\hline 1517 & 哺乳纲 & Mammalia & 食肉目 & Carnivora & 鼠科 & Mustelidae & 艾鼬 & Mustela eversmannii & & \\
\hline 1518 & 哺乳纲 & Mammalia & 食肉目 & Carnivora & 鼠科 & Mustelidae & 小艾鼣 & Mustela amurensis & $\mathrm{m}$ & 存疑种 \\
\hline 1519 & 哺乳纲 & Mammalia & 食肉目 & Carnivora & 鼠由科 & Mustelidae & 虎蝹 & Vormela peregusna & d & \\
\hline 1520 & 哺乳纲 & Mammalia & 食肉目 & Carnivora & 鼠由科 & Mustelidae & 缅甸鼠由獾 & Melogale personata & wa & \\
\hline 1521 & 哺乳纲 & Mammalia & 食肉目 & Carnivora & 鼠由科 & Mustelidae & 叟獾 & Melogale moschata & $\mathrm{sd}$ & \\
\hline 1522 & 哺乳纲 & Mammalia & 食肉目 & Carnivora & 鼠由科 & Mustelidae & 狗獾 & Meles meles & uh & 现分为 2 种 \\
\hline 1523 & 哺乳纲 & Mammalia & 食肉目 & Carnivora & 鼠科 & Mustelidae & 猪獾 & Arctonyx collaris & we & \\
\hline 1524 & 哺乳纲 & Mammalia & 食肉目 & Carnivora & 鼠科 & Mustelidae & 水獭 & Lutra lutra & uh & \\
\hline 1525 & 哺乳纲 & Mammalia & 食肉目 & Carnivora & 鼠由科 & Mustelidae & 汇獭 & Lutraogale perspicillata & wa & \\
\hline 1526 & 哺乳纲 & Mammalia & 食肉目 & Carnivora & 鼠由科 & Mustelidae & 小爪水獭 & Amblonys cinerea & wb & \\
\hline 1527 & 哺乳纲 & Mammalia & 食肉目 & Carnivora & 灵猫科 & Viverridae & 大灵猫 & Viverra zibetha & wd & \\
\hline 1528 & 哺乳纲 & Mammalia & 食肉目 & Carnivora & 灵猫科 & Viverridae & 大斑灵猫 & Viverra megaspila & wb & \\
\hline 1529 & 哺乳纲 & Mammalia & 食肉目 & Carnivora & 灵猫科 & Viverridae & 小灵猫 & Viverricula indica & wd & \\
\hline 1530 & 哺乳纲 & Mammalia & 食肉目 & Carnivora & 灵猫科 & Viverridae & 斑灵狸 & Prionodon parricolor & wc & \\
\hline 1531 & 哺乳纲 & Mammalia & 食肉目 & Carnivora & 灵猫科 & Viverridae & 梛子狸 & Paradoxurus hermaphroditus & wc & \\
\hline 1532 & 哺乳纲 & Mammalia & 食肉目 & Carnivora & 灵猫科 & Viverridae & 花面狸 & Paguma larvata & we & \\
\hline 1533 & 哺乳纲 & Mammalia & 食肉目 & Carnivora & 灵猫科 & Viverridae & 熊狸 & Arctictis binturong & wb & \\
\hline 1534 & 哺乳纲 & Mammalia & 食肉目 & Carnivora & 灵猫科 & Viverridae & 小齿狸 & Arctogalida trivirgata & wa & \\
\hline 1535 & 哺乳纲 & Mammalia & 食肉目 & Carnivora & 灵猫科 & Viverridae & 长领带狸 & Chrotogale owstoni & wa & \\
\hline 1536 & 哺乳纲 & Mammalia & 食肉目 & Carnivora & 獴科 & Herpestidae & 爪哇獴 & Herpestes javanicus & wb & \\
\hline 1537 & 哺乳纲 & Mammalia & 食肉目 & Carnivora & 㮠科 & Herpestidae & 食蟹獴 & Herpestes urva & wc & \\
\hline 1538 & 哺乳纲 & Mammalia & 食肉目 & Carnivora & 猫科 & Felidae & 野猫 & Felis silvestris & 03 & \\
\hline
\end{tabular}


郜二虎, 何杰坤, 王志臣, 徐扬, 唐小平, 江海声. 全国陆生野生动物调查单元区划方案. 生物多样性, 2017, 25 (12): 1321-1330.

http://www.biodiversity-science.net/CN/10.17520/biods.2017135

\begin{tabular}{|c|c|c|c|c|c|c|c|c|c|c|}
\hline $\begin{array}{r}\text { 序号 } \\
\text { No. }\end{array}$ & \multicolumn{2}{|c|}{$\begin{array}{c}\text { 纲 } \\
\text { Class }\end{array}$} & \multicolumn{2}{|c|}{$\begin{array}{c}\text { 目 } \\
\text { Order }\end{array}$} & \multicolumn{2}{|r|}{$\begin{array}{c}\text { 科 } \\
\text { Family }\end{array}$} & \multicolumn{2}{|r|}{$\begin{array}{l}\text { 物种名 } \\
\text { Species }\end{array}$} & \multirow{2}{*}{$\begin{array}{l}\begin{array}{c}\text { 分布型 } \\
\text { Chorotype }\end{array} \\
\mathrm{db}\end{array}$} & \multirow[t]{2}{*}{$\begin{array}{c}\text { 备注 } \\
\text { Remarks }\end{array}$} \\
\hline 1539 & 哺乳纲 & Mammalia & 食肉目 & Carnivora & 猫科 & Felidae & 漠猫 & Felis bieti & & \\
\hline 1540 & 哺乳纲 & Mammalia & 食肉目 & Carnivora & 猫科 & Felidae & 丛林猫 & Felis chaus & o & \\
\hline 1541 & 哺乳纲 & Mammalia & 食肉目 & Carnivora & 猫科 & Felidae & 兔狲 & Otocolobus manul & da & \\
\hline 1542 & 哺乳纲 & Mammalia & 食肉目 & Carnivora & 猫科 & Felidae & 金猫 & Catopuma temmincki & we & \\
\hline 1543 & 哺乳纲 & Mammalia & 食肉目 & Carnivora & 猫科 & Felidae & 豹猫 & Prionailurus bengalensis & we & \\
\hline 1544 & 哺乳纲 & Mammalia & 食肉目 & Carnivora & 猫科 & Felidae & 猞猁 & Lynx lynx & ce & \\
\hline 1545 & 哺乳纲 & Mammalia & 食肉目 & Carnivora & 猫科 & Felidae & 云猫 & Pardofelis marmorata & wc & \\
\hline 1546 & 哺乳纲 & Mammalia & 食肉目 & Carnivora & 猫科 & Felidae & 云豹 & Neofelis nebulosa & wc & \\
\hline 1547 & 哺乳纲 & Mammalia & 食肉目 & Carnivora & 猫科 & Felidae & 金钱豹 & Panthera pardus & o & \\
\hline 1548 & 哺乳纲 & Mammalia & 食肉目 & Carnivora & 猫科 & Felidae & 虎 & Panthera tigris & we & \\
\hline 1549 & 哺乳纲 & Mammalia & 食肉目 & Carnivora & 猫科 & Felidae & 雪豹 & Uncia uncia & pw & \\
\hline 1550 & 哺乳纲 & Mammalia & 长鼻目 & Proboscidea & 象科 & Elephantidae & 亚洲象 & Elephas maximus & wa & \\
\hline 1551 & 哺乳纲 & Mammalia & 奇蹄目 & Perissodactyla & 马科 & Equidae & 野马 & Equus przewalskii & $\mathrm{d}$ & \\
\hline 1552 & 哺乳纲 & Mammalia & 奇蹄目 & Perissodactyla & 马科 & Equidae & 蒙古野驴 & Equus hemionus & $\mathrm{dg}$ & \\
\hline 1553 & 哺乳纲 & Mammalia & 奇蹄目 & Perissodactyla & 马科 & Equidae & 藏野驴 & Equus kiang & pa & \\
\hline 1554 & 哺乳纲 & Mammalia & 偶蹄目 & Artiodactyla & 猪科 & Suidae & 野猪 & Sus scrofa & uh & \\
\hline 1555 & 哺乳纲 & Mammalia & 偶蹄目 & Artiodactyla & 骆驼科 & Camelidae & 野双峰驼 & Camelus ferus & de & \\
\hline 1556 & 哺乳纲 & Mammalia & 偶蹄目 & Artiodactyla & 鼠䍘鹿科 & Tragulidae & 小鼠鹿 & Tragulus javanisus & wa & \\
\hline 1557 & 哺乳纲 & Mammalia & 偶蹄目 & Artiodactyla & 㯍科 & Moschidae & 原黁 & Moschus moschiferus & $\mathrm{mg}$ & \\
\hline 1558 & 哺乳纲 & Mammalia & 偶蹄目 & Artiodactyla & 榭科 & Moschidae & 林槥 & Moschus berezovskii & sd & \\
\hline 1559 & 哺乳纲 & Mammalia & 偶蹄目 & Artiodactyla & 桖科 & Moschidae & 黑穏 & Moschus fuscus & he & \\
\hline 1560 & 哺乳纲 & Mammalia & 偶蹄目 & Artiodactyla & 瑸科 & Moschidae & 喜马拉雅榛 & Moschus leucogaster & ha & \\
\hline
\end{tabular}


郜二虎, 何杰坤, 王志臣, 徐扬, 唐小平, 江海声. 全国陆生野生动物调查单元区划方案. 生物多样性, 2017, 25 (12): 1321-1330.

http://www.biodiversity-science.net/CN/10.17520/biods.2017135

\begin{tabular}{|c|c|c|c|c|c|c|c|c|c|c|}
\hline $\begin{array}{r}\text { 序号 } \\
\text { No. }\end{array}$ & \multicolumn{2}{|c|}{$\begin{array}{c}\text { 纲 } \\
\text { Class }\end{array}$} & \multicolumn{2}{|c|}{$\begin{array}{c}\text { 目 } \\
\text { Order }\end{array}$} & \multicolumn{2}{|r|}{$\begin{array}{c}\text { 科 } \\
\text { Family }\end{array}$} & \multicolumn{2}{|r|}{$\begin{array}{l}\text { 物种名 } \\
\text { Species }\end{array}$} & \multirow{2}{*}{$\begin{array}{l}\text { 分布型 } \\
\text { Chorotype } \\
\text { sf }\end{array}$} & \multirow[t]{2}{*}{$\begin{array}{c}\text { 备注 } \\
\text { Remarks }\end{array}$} \\
\hline 1561 & 哺乳纲 & Mammalia & 偶蹄目 & Artiodactyla & 鹿科 & Cervidae & 牙獐 & Hydropotes inermis & & \\
\hline 1562 & 哺乳纲 & Mammalia & 偶蹄目 & Artiodactyla & 鹿科 & Cervidae & 毛冠鹿 & Elaphodus cephalophus & sv & \\
\hline 1563 & 哺乳纲 & Mammalia & 偶蹄目 & Artiodactyla & 鹿科 & Cervidae & 小麇 & Muntiacus reevesi & sd & \\
\hline 1564 & 哺乳纲 & Mammalia & 偶蹄目 & Artiodactyla & 鹿科 & Cervidae & 林麇 & Muntiacus feai & wb & \\
\hline 1565 & 哺乳纲 & Mammalia & 偶蹄目 & Artiodactyla & 鹿科 & Cervidae & 贡山鹿 & Muntiacus gongshanensis & $\mathrm{hm}$ & \\
\hline 1566 & 哺乳纲 & Mammalia & 偶蹄目 & Artiodactyla & 鹿科 & Cervidae & 黑麇 & Muntiacus crinifrons & si & \\
\hline 1567 & 哺乳纲 & Mammalia & 偶蹄目 & Artiodactyla & 鹿科 & Cervidae & 赤麇 & Muntiacus vaginalis & wc & \\
\hline 1568 & 哺乳纲 & Mammalia & 偶蹄目 & Artiodactyla & 鹿科 & Cervidae & 豚鹿 & Axis porcinus & wa & \\
\hline 1569 & 哺乳纲 & Mammalia & 偶蹄目 & Artiodactyla & 鹿科 & Cervidae & 水鹿 & Cervus unicolor & wd & \\
\hline 1570 & 哺乳纲 & Mammalia & 偶蹄目 & Artiodactyla & 鹿科 & Cervidae & 坡鹿 & Cervus eldi & wa & \\
\hline 1571 & 哺乳纲 & Mammalia & 偶蹄目 & Artiodactyla & 鹿科 & Cervidae & 梅花鹿 & Cervus nippon & eg & 现分为 4 种 \\
\hline 1572 & 哺乳纲 & Mammalia & 偶蹄目 & Artiodactyla & 鹿科 & Cervidae & 白唇鹿 & Cervus albirostris & $\mathrm{pc}$ & \\
\hline 1573 & 哺乳纲 & Mammalia & 偶蹄目 & Artiodactyla & 鹿科 & Cervidae & 马鹿 & Cervus elaphus & $\mathrm{cd}$ & 现分为 5 种 \\
\hline 1574 & 哺乳纲 & Mammalia & 偶蹄目 & Artiodactyla & 鹿科 & Cervidae & 魔鹿 & Elaphurus davidianus & $\mathrm{e}$ & \\
\hline 1575 & 哺乳纲 & Mammalia & 偶蹄目 & Artiodactyla & 鹿科 & Cervidae & 狍 & Capreiolus capreolus & ue & \\
\hline 1576 & 哺乳纲 & Mammalia & 偶蹄目 & Artiodactyla & 鹿科 & Cervidae & 驼鹿 & Alces alces & ca & 现分为 2 种 \\
\hline 1577 & 哺乳纲 & Mammalia & 偶蹄目 & Artiodactyla & 鹿科 & Cervidae & 驯鹿 & Rangifer tarandus & $\mathrm{ca}$ & \\
\hline 1578 & 哺乳纲 & Mammalia & 偶蹄目 & Artiodactyla & 牛科 & Bovidae & 印度野牛 & Bos gaurus & wa & \\
\hline 1579 & 哺乳纲 & Mammalia & 偶蹄目 & Artiodactyla & 牛科 & Bovidae & 爪哇野牛 & Bos javanicus & wa & \\
\hline 1580 & 哺乳纲 & Mammalia & 偶蹄目 & Artiodactyla & 牛科 & Bovidae & 野牦牛 & Bos mutus & $\mathrm{pb}$ & \\
\hline 1581 & 哺乳纲 & Mammalia & 偶蹄目 & Artiodactyla & 牛科 & Bovidae & 藏原羚 & Procapra picticaudata & pa & \\
\hline 1582 & 哺乳纲 & Mammalia & 偶蹄目 & Artiodactyla & 牛科 & Bovidae & 普氏原羚 & Procapra przewalskii & $\mathrm{dd}$ & \\
\hline
\end{tabular}


郜二虎, 何杰坤, 王志臣, 徐扬, 唐小平, 江海声. 全国陆生野生动物调查单元区划方案. 生物多样性, 2017, 25 (12): 1321-1330.

http://www.biodiversity-science.net/CN/10.17520/biods.2017135

\begin{tabular}{|c|c|c|c|c|c|c|c|c|c|c|}
\hline $\begin{array}{r}\text { 序号 } \\
\text { No. }\end{array}$ & \multicolumn{2}{|c|}{$\begin{array}{c}\text { 纲 } \\
\text { Class }\end{array}$} & \multicolumn{2}{|c|}{$\begin{array}{c}\text { 目 } \\
\text { Order }\end{array}$} & \multicolumn{2}{|r|}{$\begin{array}{c}\text { 科 } \\
\text { Family }\end{array}$} & \multicolumn{2}{|r|}{$\begin{array}{l}\text { 物种名 } \\
\text { Species }\end{array}$} & \multirow{2}{*}{$\begin{array}{l}\begin{array}{c}\text { 分布型 } \\
\text { Chorotype }\end{array} \\
\mathrm{dn}\end{array}$} & \multirow[t]{2}{*}{$\begin{array}{c}\text { 备注 } \\
\text { Remarks }\end{array}$} \\
\hline 1583 & 哺乳纲 & Mammalia & 偶蹄目 & Artiodactyla & 牛科 & Bovidae & 黄羊 & Procapra gutturosa & & \\
\hline 1584 & 哺乳纲 & Mammalia & 偶蹄目 & Artiodactyla & 牛科 & Bovidae & 我喉羚 & Gazella subgutturosa & de & \\
\hline 1585 & 哺乳纲 & Mammalia & 偶蹄目 & Artiodactyla & 牛科 & Bovidae & 藏羚 & Pantholops hodgsoni & pa & \\
\hline 1586 & 哺乳纲 & Mammalia & 偶蹄目 & Artiodactyla & 牛科 & Bovidae & 高鼻羚羊 & Saiga tatarica & $\mathrm{dc}$ & \\
\hline 1587 & 哺乳纲 & Mammalia & 偶蹄目 & Artiodactyla & 牛科 & Bovidae & 羚牛 & Budorcas taxicolor & hc & 现分为 3 种 \\
\hline 1588 & 哺乳纲 & Mammalia & 偶蹄目 & Artiodactyla & 牛科 & Bovidae & 斑羚 & Naemorhedus caudatus & $\mathrm{eb}$ & 现分为 3 种 \\
\hline 1589 & 哺乳纲 & Mammalia & 偶蹄目 & Artiodactyla & 牛科 & Bovidae & 赤斑羚 & Naemorhedus cranbrooki & he & \\
\hline 1590 & 哺乳纲 & Mammalia & 偶蹄目 & Artiodactyla & 牛科 & Bovidae & 嘉羚 & Naemorhedus sumatraensis & we & 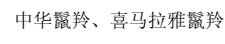 \\
\hline 1591 & 哺乳纲 & Mammalia & 偶蹄目 & Artiodactyla & 牛科 & Bovidae & 台湾鼔羚 & Naemorhedus swinhoei & $\mathrm{j}$ & \\
\hline 1592 & 哺乳纲 & Mammalia & 偶蹄目 & Artiodactyla & 牛科 & Bovidae & 喜马拉雅塔尔羊 & Hemitragus jemlahicus & ha & \\
\hline 1593 & 哺乳纲 & Mammalia & 偶蹄目 & Artiodactyla & 牛科 & Bovidae & 北山羊 & Capra ibex & pg & \\
\hline 1594 & 哺乳纲 & Mammalia & 偶蹄目 & Artiodactyla & 牛科 & Bovidae & 岩羊 & Pseudois nayaur & pa & \\
\hline 1595 & 哺乳纲 & Mammalia & 偶蹄目 & Artiodactyla & 牛科 & Bovidae & 矮岩羊 & Pseudois schaeferi & $\mathrm{pc}$ & \\
\hline 1596 & 哺乳纲 & Mammalia & 偶蹄目 & Artiodactyla & 牛科 & Bovidae & 盘羊 & Ovis ammon & pa & 现分为 5 种 \\
\hline 1597 & 哺乳纲 & Mammalia & 啮齿目 & Rodentia & 松鼠科 & Sciuridae & 北松鼠 & Sciurus vulgaris & $\mathrm{ub}$ & \\
\hline 1598 & 哺乳纲 & Mammalia & 啮齿目 & Rodentia & 松鼠科 & Sciuridae & 赤腹松鼠 & Callosciurus erythraeus & wc & \\
\hline 1599 & 哺乳纲 & Mammalia & 啮齿目 & Rodentia & 松鼠科 & Sciuridae & 金背松鼠 & Callosciurus caniceps & wa & \\
\hline 1600 & 哺乳纲 & Mammalia & 啮齿目 & Rodentia & 松鼠科 & Sciuridae & 纹腹松鼠 & Callosciurus quinquestriatus & hc & \\
\hline 1601 & 哺乳纲 & Mammalia & 啮齿目 & Rodentia & 松鼠科 & Sciuridae & 黄手松鼠 & Callosciurus phayrei & wa & \\
\hline 1602 & 哺乳纲 & Mammalia & 啮齿目 & Rodentia & 松鼠科 & Sciuridae & 蓝腹松鼠 & Callosciurus pygerythrus & wa & \\
\hline 1603 & 哺乳纲 & Mammalia & 啮齿目 & Rodentia & 松鼠科 & Sciuridae & 明纹花鼠 & Tamiops mcclellandi & wd & \\
\hline 1604 & 哺乳纲 & Mammalia & 啮齿目 & Rodentia & 松鼠科 & Sciuridae & 隐纹花鼠 & Tamiops swinhoei & we & \\
\hline
\end{tabular}


郜二虎, 何杰坤, 王志臣, 徐扬, 唐小平, 江海声. 全国陆生野生动物调查单元区划方案. 生物多样性, 2017, 25 (12): 1321-1330.

http://www.biodiversity-science.net/CN/10.17520/biods.2017135

\begin{tabular}{|c|c|c|c|c|c|c|c|c|c|c|}
\hline $\begin{array}{r}\text { 序号 } \\
\text { No. }\end{array}$ & \multicolumn{2}{|c|}{$\begin{array}{c}\text { 纲 } \\
\text { Class }\end{array}$} & \multicolumn{2}{|c|}{$\begin{array}{c}\text { 目 } \\
\text { Order }\end{array}$} & \multicolumn{2}{|r|}{$\begin{array}{c}\text { 科 } \\
\text { Family }\end{array}$} & \multicolumn{2}{|r|}{$\begin{array}{l}\text { 物种名 } \\
\text { Species }\end{array}$} & \multirow{2}{*}{$\begin{array}{l}\begin{array}{c}\text { 分布型 } \\
\text { Chorotype }\end{array} \\
\text { he }\end{array}$} & \multirow[t]{2}{*}{$\begin{array}{r}\text { 备注 } \\
\text { Remark }\end{array}$} \\
\hline 1605 & 哺乳纲 & Mammalia & 啮齿目 & Rodentia & 松鼠科 & Sciuridae & 橙腹长吻松鼠 & Dremomys lokriah & & \\
\hline 1606 & 哺乳纲 & Mammalia & 啮齿目 & Rodentia & 松鼠科 & Sciuridae & 橙喉长吻松鼠 & Dremomys gularis & wa & \\
\hline 1607 & 哺乳纲 & Mammalia & 啮齿目 & Rodentia & 松鼠科 & Sciuridae & 红腿长吻松鼠 & Dremomys pyrrhomerus & $\mathrm{sc}$ & \\
\hline 1608 & 哺乳纲 & Mammalia & 啮齿目 & Rodentia & 松鼠科 & Sciuridae & 红赖长吻松鼠 & Dremomys rufigenis & wd & \\
\hline 1609 & 哺乳纲 & Mammalia & 啮齿目 & Rodentia & 松鼠科 & Sciuridae & 珀氏长吻松鼠 & Dremomys pernyi & $\mathrm{sd}$ & \\
\hline 1610 & 哺乳纲 & Mammalia & 啮齿目 & Rodentia & 松鼠科 & Sciuridae & 巨松鼠 & Ratufa bicolor & wa & \\
\hline 1611 & 哺乳纲 & Mammalia & 啮齿目 & Rodentia & 松鼠科 & Sciuridae & 线松鼠 & Menetes berdmorei & wa & \\
\hline 1612 & 哺乳纲 & Mammalia & 啮齿目 & Rodentia & 松鼠科 & Sciuridae & 岩松鼠 & Sciurotamias davidianus & $\mathrm{e}$ & \\
\hline 1613 & 哺乳纲 & Mammalia & 啮齿目 & Rodentia & 松鼠科 & Sciuridae & 白喉岩松鼠 & Sciurotamias forresti & hc & \\
\hline 1614 & 哺乳纲 & Mammalia & 啮齿目 & Rodentia & 松鼠科 & Sciuridae & 花鼠 & Tamias sibiricus & $\mathrm{ub}$ & \\
\hline 1615 & 哺乳纲 & Mammalia & 啮齿目 & Rodentia & 松鼠科 & Sciuridae & 达乌尔黄鼠 & Spermophilus dauricus & $\mathrm{dm}$ & \\
\hline 1616 & 哺乳纲 & Mammalia & 啮齿目 & Rodentia & 松鼠科 & Sciuridae & 大黄鼠 & Spermophilus major & $\mathrm{dc}$ & 可能无分布 \\
\hline 1617 & 哺乳纲 & Mammalia & 啮齿目 & Rodentia & 松鼠科 & Sciuridae & 赤规黄鼠 & Spermophilus erythrogenys & $\mathrm{dc}$ & \\
\hline 1618 & 哺乳纲 & Mammalia & 啮齿目 & Rodentia & 松鼠科 & Sciuridae & 天山黄鼠 & Spermophilus relictus & $\mathrm{d}$ & \\
\hline 1619 & 哺乳纲 & Mammalia & 啮齿目 & Rodentia & 松鼠科 & Sciuridae & 长尾黄鼠 & Spermophilus undulatus & $\mathrm{m}$ & \\
\hline 1620 & 哺乳纲 & Mammalia & 啮齿目 & Rodentia & 松鼠科 & Sciuridae & 草原早獭 & Marmota bobak & $\mathrm{dn}$ & \\
\hline 1621 & 哺乳纲 & Mammalia & 啮齿目 & Rodentia & 松鼠科 & Sciuridae & 灰旱獭 & Marmota baibacina & $\mathrm{dp}$ & \\
\hline 1622 & 哺乳纲 & Mammalia & 啮齿目 & Rodentia & 松鼠科 & Sciuridae & 喜马拉雅早獭 & Marmota himalayana & pa & \\
\hline 1623 & 哺乳纲 & Mammalia & 啮齿目 & Rodentia & 松鼠科 & Sciuridae & 长尾旱獭 & Marmota caudata & pe & \\
\hline 1624 & 哺乳纲 & Mammalia & 啮齿目 & Rodentia & 松鼠科 & Sciuridae & 毛耳飞鼠 & Belomys pearsonii & sd & \\
\hline 1625 & 哺乳纲 & Mammalia & 啮齿目 & Rodentia & 松鼠科 & Sciuridae & 复齿鼠鼠 & Trogopterus xanthipes & $\mathrm{hm}$ & \\
\hline 1626 & 哺乳纲 & Mammalia & 啮齿目 & Rodentia & 松鼠科 & Sciuridae & 白斑小鼠鼠 & Petaurista elegans & $\mathrm{hm}$ & \\
\hline
\end{tabular}


郜二虎, 何杰坤, 王志臣, 徐扬, 唐小平, 江海声. 全国陆生野生动物调查单元区划方案. 生物多样性, 2017, 25 (12): 1321-1330.

http://www.biodiversity-science.net/CN/10.17520/biods.2017135

\begin{tabular}{|c|c|c|c|c|c|c|c|c|c|c|}
\hline $\begin{array}{r}\text { 序号 } \\
\text { No. }\end{array}$ & \multicolumn{2}{|c|}{$\begin{array}{c}\text { 纲 } \\
\text { Class }\end{array}$} & \multicolumn{2}{|c|}{$\begin{array}{c}\text { 目 } \\
\text { Order }\end{array}$} & \multicolumn{2}{|r|}{$\begin{array}{c}\text { 科 } \\
\text { Family }\end{array}$} & \multicolumn{2}{|r|}{$\begin{array}{l}\text { 物种名 } \\
\text { Species }\end{array}$} & \multirow{2}{*}{$\begin{array}{l}\begin{array}{c}\text { 分布型 } \\
\text { Chorotype }\end{array} \\
\mathrm{hm}\end{array}$} & \multirow[t]{2}{*}{$\begin{array}{c}\text { 备注 } \\
\text { Remark }\end{array}$} \\
\hline 1627 & 哺乳纲 & Mammalia & 啮齿目 & Rodentia & 松鼠科 & Sciuridae & 灰头小鼠鼠 & Petaurista caniceps & & \\
\hline 1628 & 哺乳纲 & Mammalia & 啮齿目 & Rodentia & 松鼠科 & Sciuridae & 红背鼠鼠 & Petaurista petaurista & wd & \\
\hline 1629 & 哺乳纲 & Mammalia & 啮齿目 & Rodentia & 松鼠科 & Sciuridae & 栗背大鼠鼠 & Petaurista albiventer & $\mathrm{sc}$ & \\
\hline 1630 & 哺乳纲 & Mammalia & 啮齿目 & Rodentia & 松鼠科 & Sciuridae & 霜背大鼠眥 & Petaurista philippensis & wc & \\
\hline 1631 & 哺乳纲 & Mammalia & 啮齿目 & Rodentia & 松鼠科 & Sciuridae & 丽鼠吾 & Petaurista magnificus & ha & \\
\hline 1632 & 哺乳纲 & Mammalia & 啮齿目 & Rodentia & 松鼠科 & Sciuridae & 红白鼣鼠 & Petaurista alborufus & wd & \\
\hline 1633 & 哺乳纲 & Mammalia & 啮齿目 & Rodentia & 松鼠科 & Sciuridae & 灰鼠鼠 & Petaurista xanthotis & hc & \\
\hline 1634 & 哺乳纲 & Mammalia & 啮齿目 & Rodentia & 松鼠科 & Sciuridae & 沟牙鼠䀝 & Aeretes melanopterus & hc & \\
\hline 1635 & 哺乳纲 & Mammalia & 啮齿目 & Rodentia & 松鼠科 & Sciuridae & 羊线鼠鼠 & Eupetaurus cinereus & he & \\
\hline 1636 & 哺乳纲 & Mammalia & 啮齿目 & Rodentia & 松鼠科 & Sciuridae & 小飞鼠 & Pteromys volans & uc & \\
\hline 1637 & 哺乳纲 & Mammalia & 啮齿目 & Rodentia & 松鼠科 & Sciuridae & 黑白飞鼠 & Hylopetes alboniger & wc & \\
\hline 1638 & 哺乳纲 & Mammalia & 啮齿目 & Rodentia & 河狸科 & Castoridae & 河狸 & Castor fiber & $\mathrm{u}$ & \\
\hline 1639 & 哺乳纲 & Mammalia & 啮齿目 & Rodentia & 仓鼠科 & Cricetidae & 原仓鼠 & Cricetus cricetus & ue & \\
\hline 1640 & 哺乳纲 & Mammalia & 啮齿目 & Rodentia & 仓鼠科 & Cricetidae & 黑线仓鼠 & Cricetulus barabensis & $\mathrm{xg}$ & \\
\hline 1641 & 哺乳纲 & Mammalia & 啮齿目 & Rodentia & 仓鼠科 & Cricetidae & 灰仓鼠 & Cricetulus migratorius & $\mathrm{d}$ & \\
\hline 1642 & 哺乳纲 & Mammalia & 啮齿目 & Rodentia & 仓鼠科 & Cricetidae & 长尾仓鼠 & Cricetulus longicaudatus & $\mathrm{d}$ & \\
\hline 1643 & 哺乳纲 & Mammalia & 啮齿目 & Rodentia & 仓鼠科 & Cricetidae & 藏仓鼠 & Cricetulus kamensis & pa & \\
\hline 1644 & 哺乳纲 & Mammalia & 啮齿目 & Rodentia & 仓鼠科 & Cricetidae & 大仓鼠 & Tscherskia triton & xa & \\
\hline 1645 & 哺乳纲 & Mammalia & 啮齿目 & Rodentia & 仓鼠科 & Cricetidae & 甘肃仓鼠 & Cansumys canus & o & \\
\hline 1646 & 哺乳纲 & Mammalia & 啮齿目 & Rodentia & 仓鼠科 & Cricetidae & 短尾仓鼠 & Allocricetulus eversmanni & d & \\
\hline 1647 & 哺乳纲 & Mammalia & 啮齿目 & Rodentia & 仓鼠科 & Cricetidae & 黑线毛足鼠 & Phodopus sungorus & $\mathrm{d}$ & 存疑种 \\
\hline 1648 & 哺乳纲 & Mammalia & 啮齿目 & Rodentia & 仓鼠科 & Cricetidae & 小毛足鼠 & Phodopus roborovskii & $\mathrm{dn}$ & \\
\hline
\end{tabular}


郜二虎, 何杰坤, 王志臣, 徐扬, 唐小平, 江海声. 全国陆生野生动物调查单元区划方案. 生物多样性, 2017, 25 (12): 1321-1330.

http://www.biodiversity-science.net/CN/10.17520/biods.2017135

\begin{tabular}{|c|c|c|c|c|c|c|c|c|c|c|}
\hline $\begin{array}{r}\text { 序号 } \\
\text { No. }\end{array}$ & \multicolumn{2}{|c|}{$\begin{array}{c}\text { 纲 } \\
\text { Class }\end{array}$} & \multicolumn{2}{|c|}{$\begin{array}{c}\text { 目 } \\
\text { Order }\end{array}$} & \multicolumn{2}{|r|}{$\begin{array}{c}\text { 科 } \\
\text { Family }\end{array}$} & \multicolumn{2}{|r|}{$\begin{array}{l}\text { 物种名 } \\
\text { Species }\end{array}$} & \multirow{2}{*}{$\begin{array}{l}\text { 分布型 } \\
\text { Chorotype }\end{array}$} & \multirow[t]{2}{*}{$\begin{array}{c}\text { 备注 } \\
\text { Remarks }\end{array}$} \\
\hline 1649 & 哺乳纲 & Mammalia & 啮齿目 & Rodentia & 仓鼠科 & Cricetidae & 柽柳沙鼠 & Meriones tamariscinus & & \\
\hline 1650 & 哺乳纲 & Mammalia & 啮齿目 & Rodentia & 仓鼠科 & Cricetidae & 长爪沙鼠 & Meriones unguiculatus & dn & \\
\hline 1651 & 哺乳纲 & Mammalia & 啮齿目 & Rodentia & 仓鼠科 & Cricetidae & 子午沙鼠 & Meriones meridianus & da & \\
\hline 1652 & 哺乳纲 & Mammalia & 啮齿目 & Rodentia & 仓鼠科 & Cricetidae & 红尾沙鼠 & Meriones libycus & dh & \\
\hline 1653 & 哺乳纲 & Mammalia & 啮齿目 & Rodentia & 仓鼠科 & Cricetidae & 郑氏沙鼠 & Meriones chengi & $\mathrm{d}$ & 记录有误或无分布 \\
\hline 1654 & 哺乳纲 & Mammalia & 啮齿目 & Rodentia & 仓鼠科 & Cricetidae & 短耳沙鼠 & Brachiones przewalskii & $\mathrm{db}$ & \\
\hline 1655 & 哺乳纲 & Mammalia & 啮齿目 & Rodentia & 仓鼠科 & Cricetidae & 大沙鼠 & Rhombomys opimus & $\mathrm{dc}$ & \\
\hline 1656 & 哺乳纲 & Mammalia & 啮齿目 & Rodentia & 仓鼠科 & Cricetidae & 中华鼣鼠 & Myospalax fontanierii & $\mathrm{bc}$ & \\
\hline 1657 & 哺乳纲 & Mammalia & 啮齿目 & Rodentia & 仓鼠科 & Cricetidae & 罗氏酚鼠 & Myospalax rothschildi & o & \\
\hline 1658 & 哺乳纲 & Mammalia & 啮齿目 & Rodentia & 仓鼠科 & Cricetidae & 东北盼鼠 & Myospalax psilurus & $\mathrm{bc}$ & \\
\hline 1659 & 哺乳纲 & Mammalia & 啮齿目 & Rodentia & 仓鼠科 & Cricetidae & 草原湛鼠 & Myospalax aspalax & dn & \\
\hline 1660 & 哺乳纲 & Mammalia & 啮齿目 & Rodentia & 仓鼠科 & Cricetidae & 林旅鼠 & Myopus schisticolor & uc & \\
\hline 1661 & 哺乳纲 & Mammalia & 啮齿目 & Rodentia & 仓鼠科 & Cricetidae & 鼠形田鼠 & Ellobius tancrei & $\mathrm{dc}$ & \\
\hline 1662 & 哺乳纲 & Mammalia & 啮齿目 & Rodentia & 仓鼠科 & Cricetidae & 红背鼠 & Clethrionomys rutilus & $\mathrm{c}$ & \\
\hline 1663 & 哺乳纲 & Mammalia & 啮齿目 & Rodentia & 仓鼠科 & Cricetidae & 棕背鼠 & Clethrionomys rufocanus & uc & \\
\hline 1664 & 哺乳纲 & Mammalia & 啮齿目 & Rodentia & 仓鼠科 & Cricetidae & 天山林鼠 & Clethrionomys frater & d & \\
\hline 1665 & 哺乳纲 & Mammalia & 啮齿目 & Rodentia & 仓鼠科 & Cricetidae & 黑腹线鼠 & Eothenomys melanogaster & sv & \\
\hline 1666 & 哺乳纲 & Mammalia & 啮齿目 & Rodentia & 仓鼠科 & Cricetidae & 滇绒鼠 & Eothenomys eleusis & hc & \\
\hline 1667 & 哺乳纲 & Mammalia & 啮齿目 & Rodentia & 仓鼠科 & Cricetidae & 大绒鼠 & Eothenomys miletus & hc & \\
\hline 1668 & 哺乳纲 & Mammalia & 啮齿目 & Rodentia & 仓鼠科 & Cricetidae & 克钦线鼠 & Eothenomys cachinus & he & \\
\hline 1669 & 哺乳纲 & Mammalia & 啮齿目 & Rodentia & 仓鼠科 & Cricetidae & 昭通线鼠 & Eothenomys olitor & hc & \\
\hline 1670 & 哺乳纲 & Mammalia & 啮齿目 & Rodentia & 仓鼠科 & Cricetidae & 玉龙线鼠 & Eothenomys proditor & hc & \\
\hline
\end{tabular}


郜二虎, 何杰坤, 王志臣, 徐扬, 唐小平, 江海声. 全国陆生野生动物调查单元区划方案. 生物多样性, 2017, 25 (12): 1321-1330,

http://www.biodiversity-science.net/CN/10.17520/biods.2017135

\begin{tabular}{|c|c|c|c|c|c|c|c|c|c|c|}
\hline $\begin{array}{r}\text { 序号 } \\
\text { No. }\end{array}$ & \multicolumn{2}{|c|}{$\begin{array}{c}\text { 纲 } \\
\text { Class }\end{array}$} & \multicolumn{2}{|c|}{$\begin{array}{c}\text { 目 } \\
\text { Order }\end{array}$} & \multicolumn{2}{|r|}{$\begin{array}{c}\text { 科 } \\
\text { Family }\end{array}$} & \multicolumn{2}{|r|}{$\begin{array}{l}\text { 物种名 } \\
\text { Species }\end{array}$} & \multirow{2}{*}{$\begin{array}{l}\text { 分布型 } \\
\text { Chorotype } \\
\text { hc }\end{array}$} & \multirow[t]{2}{*}{$\begin{array}{c}\text { 备注 } \\
\text { Remarks }\end{array}$} \\
\hline 1671 & 哺乳纲 & Mammalia & 啮齿目 & Rodentia & 仓鼠科 & Cricetidae & 中华线鼠 & Eothenomys chinensis & & \\
\hline 1672 & 哺乳纲 & Mammalia & 啮齿目 & Rodentia & 仓鼠科 & Cricetidae & 西南线鼠 & Eothenomys custos & he & \\
\hline 1673 & 哺乳纲 & Mammalia & 啮齿目 & Rodentia & 仓鼠科 & Cricetidae & 苛岗线鼠 & Caryomys inez & $\mathrm{bc}$ & \\
\hline 1674 & 哺乳纲 & Mammalia & 啮齿目 & Rodentia & 仓鼠科 & Cricetidae & 洮州线鼣 & Caryomys eva & hc & \\
\hline 1675 & 哺乳纲 & Mammalia & 啮齿目 & Rodentia & 仓鼠科 & Cricetidae & 斯氏高山鼠 & Alticola stoliczkanus & $\mathrm{p}$ & \\
\hline 1676 & 哺乳纲 & Mammalia & 啮齿目 & Rodentia & 仓鼠科 & Cricetidae & 库蒙高山鼠 & Alticola stracheyi & $\mathrm{p}$ & 记录有误或无分布 \\
\hline 1677 & 哺乳纲 & Mammalia & 啮齿目 & Rodentia & 仓鼠科 & Cricetidae & 扁频高山鼠 & Alticola strelzowi & di & \\
\hline 1678 & 哺乳纲 & Mammalia & 啮齿目 & Rodentia & 仓鼠科 & Cricetidae & 草原兔尾鼠 & Lagurus lagurus & $\mathrm{dc}$ & \\
\hline 1679 & 哺乳纲 & Mammalia & 啮齿目 & Rodentia & 仓鼠科 & Cricetidae & 黄兔尾鼠 & Lagurus luteus & $\mathrm{dc}$ & \\
\hline 1680 & 哺乳纲 & Mammalia & 啮齿目 & Rodentia & 仓鼠科 & Cricetidae & 水鼠 & Arvicola terrestris & u & \\
\hline 1681 & 哺乳纲 & Mammalia & 啮齿目 & Rodentia & 仓鼠科 & Cricetidae & 白尾松田鼠 & Pitymys leucurus & pa & \\
\hline 1682 & 哺乳纲 & Mammalia & 啮齿目 & Rodentia & 仓鼠科 & Cricetidae & 高原松田鼠 & Pitymys irene & $\mathrm{pf}$ & \\
\hline 1683 & 哺乳纲 & Mammalia & 啮齿目 & Rodentia & 仓鼠科 & Cricetidae & 锡金松田鼠 & Pitymys sikimensis & ha & \\
\hline 1684 & 哺乳纲 & Mammalia & 啮齿目 & Rodentia & 仓鼠科 & Cricetidae & 帕米尔松田鼠 & Pitymys juldaschi & ha & \\
\hline 1685 & 哺乳纲 & Mammalia & 啮齿目 & Rodentia & 仓鼠科 & Cricetidae & 东方田鼠 & Microtus fortis & ee & \\
\hline 1686 & 哺乳纲 & Mammalia & 啮齿目 & Rodentia & 仓鼠科 & Cricetidae & 社田鼠 & Microtus socialis & $\mathrm{dc}$ & \\
\hline 1687 & 哺乳纲 & Mammalia & 啮齿目 & Rodentia & 仓鼠科 & Cricetidae & 黑田鼠 & Microtus agrestis & $\mathrm{cb}$ & \\
\hline 1688 & 哺乳纲 & Mammalia & 啮齿目 & Rodentia & 仓鼠科 & Cricetidae & 根田鼠 & Microtus oeconomиs & ua & \\
\hline 1689 & 哺乳纲 & Mammalia & 啮齿目 & Rodentia & 仓鼠科 & Cricetidae & 莫氏田鼠 & Microtus maximowiczii & $\mathrm{x}$ & \\
\hline 1690 & 哺乳纲 & Mammalia & 啮齿目 & Rodentia & 仓鼠科 & Cricetidae & 狭颖田鼠 & Microtus gregalis & $\mathrm{u}$ & \\
\hline 1691 & 哺乳纲 & Mammalia & 啮齿目 & Rodentia & 仓鼠科 & Cricetidae & 普通田鼠 & Microtus arvalis & ub & \\
\hline 1692 & 哺乳纲 & Mammalia & 啮齿目 & Rodentia & 仓鼠科 & Cricetidae & 伊犁田鼠 & Microtus ilaeus & $\mathrm{dc}$ & \\
\hline
\end{tabular}


郜二虎, 何杰坤, 王志臣, 徐扬, 唐小平, 江海声. 全国陆生野生动物调查单元区划方案. 生物多样性, 2017, 25 (12): 1321-1330.

http://www.biodiversity-science.net/CN/10.17520/biods.2017135

\begin{tabular}{|c|c|c|c|c|c|c|c|c|c|c|}
\hline $\begin{array}{l}\text { 序号 } \\
\text { No. }\end{array}$ & \multicolumn{2}{|c|}{$\begin{array}{c}\text { 纲 } \\
\text { Class }\end{array}$} & \multicolumn{2}{|c|}{$\begin{array}{c}\text { 目 } \\
\text { Order }\end{array}$} & \multicolumn{2}{|r|}{$\begin{array}{c}\text { 科 } \\
\text { Family }\end{array}$} & \multicolumn{2}{|r|}{$\begin{array}{l}\text { 物种名 } \\
\text { Species }\end{array}$} & \multirow{2}{*}{$\begin{array}{l}\begin{array}{c}\text { 分布型 } \\
\text { Chorotype }\end{array} \\
\mathrm{pc}\end{array}$} & \multirow[t]{2}{*}{$\begin{array}{c}\text { 备注 } \\
\text { Remarks }\end{array}$} \\
\hline 1693 & 哺乳纲 & Mammalia & 啮齿目 & Rodentia & 仓鼠科 & Cricetidae & 沟牙田鼠 & Microtus bedfordi & & \\
\hline 1694 & 哺乳纲 & Mammalia & 啮齿目 & Rodentia & 仓鼠科 & Cricetidae & 川西田鼠 & Volemys musseri & he & \\
\hline 1695 & 哺乳纲 & Mammalia & 啮齿目 & Rodentia & 仓鼠科 & Cricetidae & 四川田鼠 & Volemys millicens & he & \\
\hline 1696 & 哺乳纲 & Mammalia & 啮齿目 & Rodentia & 仓鼠科 & Cricetidae & 克氏田鼠 & Volemys clarkei & he & \\
\hline 1697 & 哺乳纲 & Mammalia & 啮齿目 & Rodentia & 仓鼠科 & Cricetidae & 台湾田鼠 & Volemys kikuchii & $\mathrm{j}$ & \\
\hline 1698 & 哺乳纲 & Mammalia & 啮齿目 & Rodentia & 仓鼠科 & Cricetidae & 布氏毛足田鼠 & Lasiopodonmys brandti & dn & \\
\hline 1699 & 哺乳纲 & Mammalia & 啮齿目 & Rodentia & 仓鼠科 & Cricetidae & 棕色毛足田鼠 & Lasiopadomys mandarinus & $\mathrm{x}$ & \\
\hline 1700 & 哺乳纲 & Mammalia & 啮齿目 & Rodentia & 仓鼠科 & Cricetidae & 青海毛足田鼠 & Lasiopodonmys fuscus & $\mathrm{p}$ & \\
\hline 1701 & 哺乳纲 & Mammalia & 啮齿目 & Rodentia & 鼠科 & Muridae & 长尾攀鼠 & Vandeleuria oleracea & wa & \\
\hline 1702 & 哺乳纲 & Mammalia & 啮齿目 & Rodentia & 鼠科 & Muridae & 小狘鼠 & Hapalomys delacouri & wa & \\
\hline 1703 & 哺乳纲 & Mammalia & 啮齿目 & Rodentia & 鼠科 & Muridae & 长尾线鼠 & Hapalomys longicaudatus & wa & \\
\hline 1704 & 哺乳纲 & Mammalia & 啮齿目 & Rodentia & 鼠科 & Muridae & 笔尾树鼠 & Chiropodomys gliroides & wa & \\
\hline 1705 & 哺乳纲 & Mammalia & 啮齿目 & Rodentia & 鼠科 & Muridae & 景东笔尾树鼠 & Chiropodomys jingdongensis & hc & 记录有误或无分布 \\
\hline 1706 & 哺乳纲 & Mammalia & 啮齿目 & Rodentia & 鼠科 & Muridae & 滇攀鼠 & Vernaya fulva & hc & \\
\hline 1707 & 哺乳纲 & Mammalia & 啮齿目 & Rodentia & 鼠科 & Muridae & 巢鼠 & Micromys minutus & uh & \\
\hline 1708 & 哺乳纲 & Mammalia & 啮齿目 & Rodentia & 鼠科 & Muridae & 小林姬鼠 & Apodemus sylvaticus & $\mathrm{u}$ & \\
\hline 1709 & 哺乳纲 & Mammalia & 啮齿目 & Rodentia & 鼠科 & Muridae & 大林姬鼠 & Apodemus peninsulae & $\mathrm{x}$ & \\
\hline 1710 & 哺乳纲 & Mammalia & 啮齿目 & Rodentia & 鼠科 & Muridae & 大耳姬鼠 & Apodemus latronum & hc & \\
\hline 1711 & 哺乳纲 & Mammalia & 啮齿目 & Rodentia & 鼠科 & Muridae & 中华姬鼠 & Apodemus draco & sd & \\
\hline 1712 & 哺乳纲 & Mammalia & 啮齿目 & Rodentia & 鼠科 & Muridae & 黑线姬鼠 & Apodemus agrarius & ub & \\
\hline 1713 & 哺乳纲 & Mammalia & 啮齿目 & Rodentia & 鼠科 & Muridae & 高山姬鼠 & Apodemus chevrieri & $\mathrm{sb}$ & \\
\hline 1714 & 哺乳纲 & Mammalia & 啮齿目 & Rodentia & 鼠科 & Muridae & 大齿鼠 & Dacnomys millardi & wa & \\
\hline
\end{tabular}


郜二虎, 何杰坤, 王志臣, 徐扬, 唐小平, 江海声. 全国陆生野生动物调查单元区划方案. 生物多样性, 2017, 25 (12): 1321-1330.

http://www.biodiversity-science.net/CN/10.17520/biods.2017135

\begin{tabular}{|c|c|c|c|c|c|c|c|c|c|c|}
\hline $\begin{array}{r}\text { 序号 } \\
\text { No. }\end{array}$ & \multicolumn{2}{|c|}{$\begin{array}{c}\text { 纲 } \\
\text { Class }\end{array}$} & \multicolumn{2}{|c|}{$\begin{array}{c}\text { 目 } \\
\text { Order }\end{array}$} & \multicolumn{2}{|r|}{$\begin{array}{c}\text { 科 } \\
\text { Family }\end{array}$} & \multicolumn{2}{|r|}{$\begin{array}{l}\text { 物种名 } \\
\text { Species }\end{array}$} & \multirow{2}{*}{$\begin{array}{l}\begin{array}{c}\text { 分布型 } \\
\text { Chorotype }\end{array} \\
\text { we }\end{array}$} & \multirow[t]{2}{*}{$\begin{array}{c}\text { 备注 } \\
\text { Remarks }\end{array}$} \\
\hline 1715 & 哺乳纲 & Mammalia & 啮齿目 & Rodentia & 鼠科 & Muridae & 黄胸鼠 & Rattus tanezumi & & \\
\hline 1716 & 哺乳纲 & Mammalia & 啮齿目 & Rodentia & 鼠科 & Muridae & 大足鼠 & Rattus nitidus & wa & \\
\hline 1717 & 哺乳纲 & Mammalia & 啮齿目 & Rodentia & 鼠科 & Muridae & 小缅鼠 & Rattus exulans & wa & \\
\hline 1718 & 哺乳纲 & Mammalia & 啮齿目 & Rodentia & 鼠科 & Muridae & 褐家鼠 & Rattus norvegicus & ue & \\
\hline 1719 & 哺乳纲 & Mammalia & 啮齿目 & Rodentia & 鼠科 & Muridae & 社鼠 & Niviventer confucianus & we & \\
\hline 1720 & 哺乳纲 & Mammalia & 啮齿目 & Rodentia & 鼠科 & Muridae & 安氏白腹鼠 & Niviventer andersoni & wd & \\
\hline 1721 & 哺乳纲 & Mammalia & 啮齿目 & Rodentia & 鼠科 & Muridae & 刺毛鼠 & Niviventer fulvescens & wb & \\
\hline 1722 & 哺乳纲 & Mammalia & 啮齿目 & Rodentia & 鼠科 & Muridae & 褐尾鼠 & Niviventer cremoriventer & wb & \\
\hline 1723 & 哺乳纲 & Mammalia & 啮齿目 & Rodentia & 鼠科 & Muridae & 灰腹鼠 & Niviventer eha & $\mathrm{hm}$ & \\
\hline 1724 & 哺乳纲 & Mammalia & 啮齿目 & Rodentia & 鼠科 & Muridae & 王鼠 & Maxomys rajah & wa & \\
\hline 1725 & 哺乳纲 & Mammalia & 啮齿目 & Rodentia & 鼠科 & Muridae & 短尾锋毛鼠 & Maxomys musschenbroeki & wb & \\
\hline 1726 & 哺乳纲 & Mammalia & 啮齿目 & Rodentia & 鼠科 & Muridae & 青毛硕鼠 & Berylmys bowersi & wc & \\
\hline 1727 & 哺乳纲 & Mammalia & 啮齿目 & Rodentia & 鼠科 & Muridae & 大泡硕鼠 & Berylmys berdmorei & wa & \\
\hline 1728 & 哺乳纲 & Mammalia & 啮齿目 & Rodentia & 鼠科 & Muridae & 小泡硕鼠 & Berylmys manipulus & he & \\
\hline 1729 & 哺乳纲 & Mammalia & 啮齿目 & Rodentia & 鼠科 & Muridae & 白腹巨鼠 & Leopoldamys edwardsi & wd & \\
\hline 1730 & 哺乳纲 & Mammalia & 啮齿目 & Rodentia & 鼠科 & Muridae & 小家鼠 & Mus musculus & uh & \\
\hline 1731 & 哺乳纲 & Mammalia & 啮齿目 & Rodentia & 鼠科 & Muridae & 卡氏小鼠 & Mus caroli & wb & \\
\hline 1732 & 哺乳纲 & Mammalia & 啮齿目 & Rodentia & 鼠科 & Muridae & 锡金小鼠 & Mus pahari & wc & \\
\hline 1733 & 哺乳纲 & Mammalia & 啮齿目 & Rodentia & 鼠科 & Muridae & 仔鹿小鼠 & Mus cervicolor & wa & \\
\hline 1734 & 哺乳纲 & Mammalia & 啮齿目 & Rodentia & 鼠科 & Muridae & 板齿鼠 & Bandicota indica & wa & \\
\hline 1735 & 哺乳纲 & Mammalia & 啮齿目 & Rodentia & 刺山鼠科 & Platacanthomyidae & 猪尾鼠 & Typhlomys cinereus & sd & \\
\hline 1736 & 哺乳纲 & Mammalia & 啮齿目 & Rodentia & 竹鼠科 & Rhizomyidae & 小竹鼠 & Cannomys badius & he & \\
\hline
\end{tabular}


郜二虎, 何杰坤, 王志臣, 徐扬, 唐小平, 江海声. 全国陆生野生动物调查单元区划方案. 生物多样性, 2017, 25 (12): 1321-1330.

http://www.biodiversity-science.net/CN/10.17520/biods.2017135

\begin{tabular}{|c|c|c|c|c|c|c|c|c|c|c|}
\hline $\begin{array}{r}\text { 序号 } \\
\text { No. }\end{array}$ & \multicolumn{2}{|c|}{$\begin{array}{c}\text { 纲 } \\
\text { Class }\end{array}$} & \multicolumn{2}{|c|}{$\begin{array}{c}\text { 目 } \\
\text { Order }\end{array}$} & \multicolumn{2}{|r|}{$\begin{array}{c}\text { 科 } \\
\text { Family }\end{array}$} & \multicolumn{2}{|r|}{$\begin{array}{l}\text { 物种名 } \\
\text { Species }\end{array}$} & \multirow{2}{*}{$\begin{array}{l}\begin{array}{c}\text { 分布型 } \\
\text { Chorotype }\end{array} \\
\text { we }\end{array}$} & \multirow[t]{2}{*}{$\begin{array}{c}\text { 备注 } \\
\text { Remarks }\end{array}$} \\
\hline 1737 & 哺乳纲 & Mammalia & 啮齿目 & Rodentia & 竹鼠科 & Rhizomyidae & 中华竹鼠 & Rhizomys sinensis & & \\
\hline 1738 & 哺乳纲 & Mammalia & 啮齿目 & Rodentia & 竹鼠科 & Rhizomyidae & 银星竹鼠 & Rhizomys pruinosus & $\mathrm{wb}$ & \\
\hline 1739 & 哺乳纲 & Mammalia & 啮齿目 & Rodentia & 竹鼠科 & Rhizomyidae & 大竹鼠 & Rhizomys sumatrensis & wa & \\
\hline 1740 & 哺乳纲 & Mammalia & 啮齿目 & Rodentia & 睡鼠科 & Myoxidae & 林睡鼠 & Dryomys nitedula & u & \\
\hline 1741 & 哺乳纲 & Mammalia & 啮齿目 & Rodentia & 睡鼠科 & Myoxidae & 四川毛尾睡鼠 & Chaetocauda sichuanensis & hc & \\
\hline 1742 & 哺乳纲 & Mammalia & 啮齿目 & Rodentia & 跳鼠科 & Dipodidae & 草原蹶鼠 & Sicista subtilis & o & \\
\hline 1743 & 哺乳纲 & Mammalia & 啮齿目 & Rodentia & 跳鼠科 & Dipodidae & 跖鼠 & Sicista concolor & $\mathrm{u}$ & \\
\hline 1744 & 哺乳纲 & Mammalia & 啮齿目 & Rodentia & 跳鼠科 & Dipodidae & 四川林跳鼠 & Eozapus setchuanus & hc & \\
\hline 1745 & 哺乳纲 & Mammalia & 啮齿目 & Rodentia & 跳鼠科 & Dipodidae & 五趾跳鼠 & Allactaga sibirica & $\mathrm{dc}$ & \\
\hline 1746 & 哺乳纲 & Mammalia & 啮齿目 & Rodentia & 跳鼠科 & Dipodidae & 小五趾跳鼠 & Allactaga elater & $\mathrm{dc}$ & \\
\hline 1747 & 哺乳纲 & Mammalia & 啮齿目 & Rodentia & 跳鼠科 & Dipodidae & 巨泡五趾跳鼠 & Allactaga bullata & da & \\
\hline 1748 & 哺乳纲 & Mammalia & 啮齿目 & Rodentia & 跳鼠科 & Dipodidae & 小地兔 & Pygeretmus pumilio & $\mathrm{dc}$ & \\
\hline 1749 & 哺乳纲 & Mammalia & 啮齿目 & Rodentia & 跳鼠科 & Dipodidae & 五趾心颎跳鼠 & Cardiocranius paradoxus & $\mathrm{dc}$ & \\
\hline 1750 & 哺乳纲 & Mammalia & 啮齿目 & Rodentia & 跳鼠科 & Dipodidae & 三趾心颎跳鼠 & Salpingotus kozlovi & $\mathrm{db}$ & \\
\hline 1751 & 哺乳纲 & Mammalia & 啮齿目 & Rodentia & 跳鼠科 & Dipodidae & 肥尾心顽跳鼠 & Salpingotus crassicauda & $\mathrm{dc}$ & \\
\hline 1752 & 哺乳纲 & Mammalia & 啮齿目 & Rodentia & 跳鼠科 & Dipodidae & 三趾跳鼠 & Dipus sagitta & $\mathrm{dg}$ & \\
\hline 1753 & 哺乳纲 & Mammalia & 啮齿目 & Rodentia & 跳鼠科 & Dipodidae & 羽尾跳鼠 & Stolodipus telum & $\mathrm{dc}$ & \\
\hline 1754 & 哺乳纲 & Mammalia & 啮齿目 & Rodentia & 跳鼠科 & Dipodidae & 长耳跳鼠 & Euchoreutes naso & da & \\
\hline 1755 & 哺乳纲 & Mammalia & 啮齿目 & Rodentia & 豪猪科 & Hystricidae & 帚尾豪猪 & Atherurus macrourus & wc & \\
\hline 1756 & 哺乳纲 & Mammalia & 啮齿目 & Rodentia & 豪猪科 & Hystricidae & 马来豪猪 & Hystrix brachyura & wd & \\
\hline 1757 & 哺乳纲 & Mammalia & 兔形目 & Lagomorpha & 鼠兔科 & Ochotonidae & 突频鼠兔 & Ochotona koslowi & $\mathrm{pg}$ & \\
\hline 1758 & 哺乳纲 & Mammalia & 兔形目 & Lagomorpha & 鼠兔科 & Ochotonidae & 黑唇鼠兔 & Ochotona curzoniae & $\mathrm{p}$ & \\
\hline
\end{tabular}


郜二虎, 何杰坤, 王志臣, 徐扬, 唐小平, 江海声. 全国陆生野生动物调查单元区划方案. 生物多样性, 2017, 25 (12): 1321-1330.

http://www.biodiversity-science.net/CN/10.17520/biods.2017135

\begin{tabular}{|c|c|c|c|c|c|c|c|c|c|c|}
\hline $\begin{array}{r}\text { 序号 } \\
\text { No. }\end{array}$ & \multicolumn{2}{|c|}{$\begin{array}{c}\text { 纲 } \\
\text { Class }\end{array}$} & \multicolumn{2}{|c|}{$\begin{array}{c}\text { 目 } \\
\text { Order }\end{array}$} & \multicolumn{2}{|r|}{$\begin{array}{c}\text { 科 } \\
\text { Family }\end{array}$} & \multicolumn{2}{|r|}{$\begin{array}{l}\text { 物种名 } \\
\text { Species }\end{array}$} & \multirow{2}{*}{$\begin{array}{l}\begin{array}{c}\text { 分布型 } \\
\text { Chorotype }\end{array} \\
\mathrm{dn}\end{array}$} & \multirow[t]{2}{*}{$\begin{array}{c}\text { 备注 } \\
\text { Remarks }\end{array}$} \\
\hline 1759 & 哺乳纲 & Mammalia & 兔形目 & Lagomorpha & 鼠兔科 & Ochotonidae & 达乌尔鼠兔 & Ochotona daurica & & \\
\hline 1760 & 哺乳纲 & Mammalia & 兔形目 & Lagomorpha & 鼠兔科 & Ochotonidae & 喜马拉雅鼠兔 & Ochotona himalayana & ha & \\
\hline 1761 & 哺乳纲 & Mammalia & 兔形目 & Lagomorpha & 鼠兔科 & Ochotonidae & 伊犁鼠兔 & Ochotona iliensis & d & \\
\hline 1762 & 哺乳纲 & Mammalia & 兔形目 & Lagomorpha & 鼠兔科 & Ochotonidae & 大耳鼠兔 & Ochotona macrotis & pa & \\
\hline 1763 & 哺乳纲 & Mammalia & 兔形目 & Lagomorpha & 鼠兔科 & Ochotonidae & 灰鼠兔 & Ochotona roylei & $\mathrm{hm}$ & \\
\hline 1764 & 哺乳纲 & Mammalia & 兔形目 & Lagomorpha & 鼠兔科 & Ochotonidae & 努布拉鼠兔 & Ochotona nubrica & pa & \\
\hline 1765 & 哺乳纲 & Mammalia & 兔形目 & Lagomorpha & 鼠兔科 & Ochotonidae & 灰颈鼠兔 & Ochotona forresti & he & \\
\hline 1766 & 哺乳纲 & Mammalia & 兔形目 & Lagomorpha & 鼠兔科 & Ochotonidae & 高黎贡鼠兔 & Ochotona gaoligongensis & hc & \\
\hline 1767 & 哺乳纲 & Mammalia & 兔形目 & Lagomorpha & 鼠兔科 & Ochotonidae & 黄河鼠兔 & Ochotona huangensis & $\mathrm{pf}$ & \\
\hline 1768 & 哺乳纲 & Mammalia & 兔形目 & Lagomorpha & 鼠兔科 & Ochotonidae & 藏鼠兔 & Ochotona thibetana & hc & \\
\hline 1769 & 哺乳纲 & Mammalia & 兔形目 & Lagomorpha & 鼠兔科 & Ochotonidae & 间领鼠兔 & Ochotona cansus & $\mathrm{pc}$ & \\
\hline 1770 & 哺乳纲 & Mammalia & 兔形目 & Lagomorpha & 鼠兔科 & Ochotonidae & 狭㐿鼠兔 & Ochotona thomasi & $\mathrm{pc}$ & \\
\hline 1771 & 哺乳纲 & Mammalia & 兔形目 & Lagomorpha & 鼠兔科 & Ochotonidae & 川西鼠兔 & Ochotona gloveri & $\mathrm{pc}$ & \\
\hline 1772 & 哺乳纲 & Mammalia & 兔形目 & Lagomorpha & 鼠兔科 & Ochotonidae & 高山鼠兔 & Ochotona alpina & o & 现分为 3 种 \\
\hline 1773 & 哺乳纲 & Mammalia & 兔形目 & Lagomorpha & 鼠兔科 & Ochotonidae & 帕氏鼠兔 & Ochotona pallasi & d & \\
\hline 1774 & 哺乳纲 & Mammalia & 兔形目 & Lagomorpha & 鼠兔科 & Ochotonidae & 拉达克鼠兔 & Ochotona ladacensis & pe & \\
\hline 1775 & 哺乳纲 & Mammalia & 兔形目 & Lagomorpha & 鼠兔科 & Ochotonidae & 红耳鼠兔 & Ochotona erythrotis & $\mathrm{pf}$ & \\
\hline 1776 & 哺乳纲 & Mammalia & 兔形目 & Lagomorpha & 鼠兔科 & Ochotonidae & 红鼠兔 & Ochotona rutila & o & \\
\hline 1777 & 哺乳纲 & Mammalia & 兔形目 & Lagomorpha & 兔科 & Leporidae & 雪兔 & Lepus timidus & $\mathrm{ca}$ & \\
\hline 1778 & 哺乳纲 & Mammalia & 兔形目 & Lagomorpha & 兔科 & Leporidae & 东北兔 & Lepus mandshuricus & $\mathrm{mb}$ & \\
\hline 1779 & 哺乳纲 & Mammalia & 兔形目 & Lagomorpha & 兔科 & Leporidae & 华南兔 & Lepus sinensis & $\mathrm{sc}$ & \\
\hline 1780 & 哺乳纲 & Mammalia & 兔形目 & Rodentla & 兔科 & Leporidae & 草兔 & Lepus capensis & o & 蒙古兔、中亚兔 \\
\hline
\end{tabular}


郜二虎, 何杰坤, 王志臣, 徐扬, 唐小平, 江海声. 全国陆生野生动物调查单元区划方案. 生物多样性, 2017, 25 (12): 1321-1330.

http://www.biodiversity-science.net/CN/10.17520/biods.2017135

\begin{tabular}{|c|c|c|c|c|c|c|c|c|c|c|}
\hline $\begin{array}{l}\text { 序号 } \\
\text { No. }\end{array}$ & \multicolumn{2}{|c|}{$\begin{array}{c}\text { 纲 } \\
\text { Class }\end{array}$} & \multicolumn{2}{|c|}{$\begin{array}{c}\text { 目 } \\
\text { Order }\end{array}$} & \multicolumn{2}{|r|}{$\begin{array}{c}\text { 科 } \\
\text { Family }\end{array}$} & \multicolumn{2}{|r|}{$\begin{array}{l}\text { 物种名 } \\
\text { Species }\end{array}$} & \multirow{2}{*}{$\begin{array}{c}\begin{array}{c}\text { 分布型 } \\
\text { Chorotype }\end{array} \\
\mathrm{pa}\end{array}$} & \multirow[t]{2}{*}{$\begin{array}{c}\text { 备注 } \\
\text { Remarks } \\
\end{array}$} \\
\hline 1781 & 哺乳纲 & Mammalia & 兔形目 & Rodentla & 兔科 & Leporidae & 高原兔 & Lepus oiostolus & & \\
\hline 1782 & 哺乳纲 & Mammalia & 兔形目 & Rodentla & 兔科 & Leporidae & 云南兔 & Lepus сотиs & yc & \\
\hline 1783 & 哺乳纲 & Mammalia & 兔形目 & Rodentla & 兔科 & Leporidae & 海南兔 & Lepus hainanus & j & \\
\hline 1784 & 哺乳纲 & Mammalia & 兔形目 & Rodentla & 兔科 & Leporidae & 塔里木兔 & Lepus yarkandensis & $\mathrm{db}$ & \\
\hline
\end{tabular}


郜二虎, 何杰坤, 王志臣, 徐扬, 唐小平, 江海声. 全国陆生野生动物调查单元区划方案. 生物多样性, 2017, 25 (12): 1321-1330.

http://www.biodiversity-science.net/CN/10.17520/biods.2017135

附录 6 分布型代码

Appendix 6 Code of chorotype

\begin{tabular}{|c|c|c|c|}
\hline $\begin{array}{c}\text { 一级型代码 } \\
\text { Code }\end{array}$ & $\begin{array}{c}\text { 一级型 } \\
\text { Chorotype name }\end{array}$ & $\begin{array}{c}\text { 二级型代码 } \\
\text { Code }\end{array}$ & $\begin{array}{c}\text { 二级型 } \\
\text { Chorotype name }\end{array}$ \\
\hline \multirow[t]{16}{*}{$\mathrm{C}$} & \multirow[t]{16}{*}{ 全北型 Holarctic Type } & $\mathrm{C}$ & 全北型 \\
\hline & & $\mathrm{Ca}$ & 寒带至寒温带(苔原-针叶林带) \\
\hline & & $\mathrm{Cb}$ & $\begin{array}{l}\text { 寒温带至中温带(针叶林带-森林草 } \\
\text { 原) }\end{array}$ \\
\hline & & $\mathrm{Cc}$ & 寒温带(针叶林带)为主 \\
\hline & & $\mathrm{Cd}$ & $\begin{array}{l}\text { 温带(落叶阔叶林带-草原耕作景 } \\
\text { 观) }\end{array}$ \\
\hline & & $\mathrm{Ce}$ & 北方湿润-半湿润带 \\
\hline & & $\mathrm{Cf}$ & 中温带为主 \\
\hline & & $\mathrm{Cg}$ & $\begin{array}{l}\text { 中温带为主, 再伸至亚热带(欧亚 } \\
\text { 温带-亚热带型) }\end{array}$ \\
\hline & & $\mathrm{Ch}$ & $\begin{array}{l}\text { 温带为主, 再伸至热带(欧亚温带- } \\
\text { 热带型) }\end{array}$ \\
\hline & & $\mathrm{Cp}$ & 太平洋 \\
\hline & & $\mathrm{Ci}$ & 印度洋-太平洋 \\
\hline & & $\mathrm{Cw}$ & 海洋 \\
\hline & & Cwl & 海洋+世界性 \\
\hline & & Cwp & 海洋+太平洋 \\
\hline & & Cwn & 海洋+北极圈 \\
\hline & & Cwi & 海洋+印度洋-太平洋 \\
\hline \multirow[t]{13}{*}{$\mathrm{U}$} & \multirow[t]{13}{*}{ 古北型 Palearctic type } & $\mathrm{U}$ & 古北型 \\
\hline & & $\mathrm{Ua}$ & 寒带至寒温带(苔原-针叶林带) \\
\hline & & $\mathrm{Ub}$ & $\begin{array}{l}\text { 寒温带至中温带(针叶林带-森林草 } \\
\text { 原) }\end{array}$ \\
\hline & & Uc & 寒温带(针叶林带)为主 \\
\hline & & Ud & $\begin{array}{l}\text { 温带(落叶阔叶林带-草原耕作景 } \\
\text { 观) }\end{array}$ \\
\hline & & $\mathrm{Ue}$ & 北方湿润-半湿润带 \\
\hline & & Uf & 中温带为主 \\
\hline & & $\mathrm{Ug}$ & $\begin{array}{l}\text { 中温带为主, 再伸至亚热带(欧亚 } \\
\text { 温带-亚热带型) }\end{array}$ \\
\hline & & Uh & $\begin{array}{l}\text { 温带为主, 再伸至热带(欧亚温带- } \\
\text { 热带型) }\end{array}$ \\
\hline & & Uw & 海洋 \\
\hline & & Up & 太平洋 \\
\hline & & Uwl & 海洋+世界性 \\
\hline & & Uwp & 海洋+太平洋 \\
\hline \multirow[t]{2}{*}{ A } & \multirow{2}{*}{$\begin{array}{l}\text { 澳大利亚-东南亚群岛 Australia-Southeast } \\
\text { Asian Islands Type }\end{array}$} & A & 澳大利亚-东南亚群岛 \\
\hline & & Aw & 海洋 \\
\hline
\end{tabular}


郜二虎, 何杰坤, 王志臣, 徐扬, 唐小平, 江海声. 全国陆生野生动物调查单元区划方案. 生物多样性, 2017, 25 (12): 1321-1330.

http://www.biodiversity-science.net/CN/10.17520/biods.2017135

\begin{tabular}{|c|c|c|c|}
\hline $\begin{array}{c}\text { 一级型代码 } \\
\text { Code }\end{array}$ & $\begin{array}{c}\text { 一级型 } \\
\text { Chorotype name }\end{array}$ & $\begin{array}{c}\text { 二级型代码 } \\
\text { Code }\end{array}$ & $\begin{array}{c}\text { 二级型 } \\
\text { Chorotype name }\end{array}$ \\
\hline & & Ap & 太平洋 \\
\hline & & $\mathrm{Ai}$ & 印度洋-太平洋 \\
\hline & & $\mathrm{Al}$ & 世界性 \\
\hline & & An & 北极圈 \\
\hline & & Awp & 海洋+太平洋 \\
\hline \multirow[t]{8}{*}{ M } & \multirow{8}{*}{$\begin{array}{l}\text { 东北型(我国东北部地区或再包括附近地区) } \\
\text { Northeast China Type (Species distributed in } \\
\text { Northeast China or nearby) }\end{array}$} & M & $\begin{array}{l}\text { 东北型(我国东北部地区或再包括 } \\
\text { 附近地区) }\end{array}$ \\
\hline & & $\mathrm{Ma}$ & $\begin{array}{l}\text { 包括贝加尔、蒙古、阿穆尔、乌苏 } \\
\text { 里(或部分, 下同) }\end{array}$ \\
\hline & & $\mathrm{Mb}$ & 包括乌苏里及朝鲜半岛 \\
\hline & & $\mathrm{Mc}$ & 包括朝鲜半岛 \\
\hline & & Md & 再分布至蒙古 \\
\hline & & $\mathrm{Me}$ & 包括朝鲜半岛和蒙古 \\
\hline & & Mf & 包括朝鲜半岛、乌苏里及远东地区 \\
\hline & & $\mathrm{Mg}$ & 包括乌苏里及东西伯利亚 \\
\hline \multirow[t]{7}{*}{ K } & \multirow{7}{*}{$\begin{array}{l}\text { 东北型 (东部为主) Northeast China Type } \\
\text { (Species mainly distributed in east of Northeast } \\
\text { China) }\end{array}$} & K & 东北型(东部为主) \\
\hline & & $\mathrm{Ka}$ & $\begin{array}{l}\text { 包括阿穆尔、东西伯利亚、乌苏里、 } \\
\text { 朝鲜半岛 }\end{array}$ \\
\hline & & $\mathrm{Kb}$ & 包括乌苏里及朝鲜半岛 \\
\hline & & Kc & 包括 $a 、 b$ 及俄罗斯远东 \\
\hline & & $\mathrm{Kd}$ & 包括朝鲜半岛 \\
\hline & & $\mathrm{Ke}$ & 包括西伯利亚及乌苏里 \\
\hline & & $\mathrm{Kf}$ & 包括朝鲜半岛及日本 \\
\hline \multirow[t]{4}{*}{ B } & \multirow{4}{*}{$\begin{array}{l}\text { 华北型(主要分布于华北区) North China } \\
\text { Type (Species mainly distributed in Northern } \\
\text { China) }\end{array}$} & B & 华北型(主要分布于华北区) \\
\hline & & $\mathrm{Ba}$ & 还包括周边地区 \\
\hline & & $\mathrm{Bb}$ & 主要分布在东部 \\
\hline & & $\mathrm{Bc}$ & 主要分布在西部 \\
\hline \multirow[t]{8}{*}{$\mathrm{X}$} & \multirow[t]{8}{*}{ 东北-华北型 North- Northeast China Type } & $\mathrm{X}$ & 东北-华北型 \\
\hline & & $\mathrm{Xa}$ & 再包括阿穆尔、乌苏里、朝鲜半岛 \\
\hline & & $\mathrm{Xb}$ & $\begin{array}{l}\text { 再包括乌苏里、朝鲜半岛、俄罗斯 } \\
\text { 远东 }\end{array}$ \\
\hline & & $\mathrm{Xc}$ & 再包括朝鲜半岛 \\
\hline & & $\mathrm{Xd}$ & 伸展至蒙古 \\
\hline & & $\mathrm{Xe}$ & 伸展至朝鲜半岛与蒙古 \\
\hline & & $\mathrm{Xf}$ & 伸展至朝鲜半岛及俄罗斯远东 \\
\hline & & $\mathrm{Xg}$ & $\begin{array}{l}\text { 伸展至阿穆尔、乌苏里、蒙古东部、 } \\
\text { 贝加尔 }\end{array}$ \\
\hline \multirow[t]{3}{*}{$\mathrm{E}$} & \multirow{3}{*}{$\begin{array}{l}\text { 季风区型(东部湿润地区为主) Monsoon } \\
\text { Region Type (Species mainly distributed in } \\
\text { Eastern Humid Region) }\end{array}$} & E & 季风区型(东部湿润地区为主) \\
\hline & & $\mathrm{Ea}$ & $\begin{array}{l}\text { 包括阿穆尔或再延展至俄罗斯远 } \\
\text { 东地区 }\end{array}$ \\
\hline & & $\mathrm{Eb}$ & 包括乌苏里或再延展至朝鲜及俄 \\
\hline
\end{tabular}


郜二虎，何杰坤，王志臣，徐扬，唐小平，江海声. 全国陆生野生动物调查单元区划方案. 生物多样性, 2017, 25 (12): 1321-1330.

http://www.biodiversity-science.net/CN/10.17520/biods.2017135

\begin{tabular}{|c|c|c|c|}
\hline $\begin{array}{c}\text { 一级型代码 } \\
\text { Code }\end{array}$ & $\begin{array}{c}\text { 一级型 } \\
\text { Chorotype name }\end{array}$ & $\begin{array}{c}\text { 二级型代码 } \\
\text { Code }\end{array}$ & $\begin{array}{c}\text { 二级型 } \\
\text { Chorotype name }\end{array}$ \\
\hline & & & 罗斯远东 \\
\hline & & $\mathrm{Ec}$ & 包括蒙古及贝加尔湖地区 \\
\hline & & Ed & 包括至朝鲜及日本 \\
\hline & & $\mathrm{Ee}$ & 包括蒙古、贝加尔与朝鲜 \\
\hline & & Ef & $\begin{array}{l}\text { 包括上述大部分地区更至西伯利 } \\
\text { 亚 }\end{array}$ \\
\hline & & Eg & 包括乌苏里、朝鲜 \\
\hline & & Eh & 包括俄罗斯远东地区、日本 \\
\hline \multirow[t]{14}{*}{$\mathrm{D}$} & \multirow{14}{*}{$\begin{array}{l}\text { 中亚型(中亚温带干旱区分布) Central Asia } \\
\text { Type (Species distributed in Central Asian } \\
\text { Temperate Arid Zone) }\end{array}$} & $\mathrm{D}$ & 中亚型(中亚温带干旱区分布) \\
\hline & & $\mathrm{Da}$ & 塔里木-准噶尔或再包括附近地区 \\
\hline & & $\mathrm{Db}$ & 塔里木为主或再包括附近地区 \\
\hline & & $\mathrm{Dc}$ & 准噶尔为主或再包括附近地区 \\
\hline & & Dd & 阿拉善为主 \\
\hline & & $\mathrm{De}$ & $\begin{array}{l}a+b \text { 再包括柴达木或更包括青海湖 } \\
\text { 盆地区 }\end{array}$ \\
\hline & & Df & 伸展至天山或附近地区 \\
\hline & & $\mathrm{Dg}$ & $\begin{array}{l}\text { a, 再包括柴达木或更包括青海湖 } \\
\text { 盆. }\end{array}$ \\
\hline & & $\mathrm{Dh}$ & 伊犁地区为主 \\
\hline & & Di & 阿尔泰山地或更包括附近地区 \\
\hline & & $\mathrm{Dm}$ & 塔城一带 \\
\hline & & $\mathrm{Dk}$ & $\mathrm{i}+\mathrm{m}$ \\
\hline & & Dn & 内蒙古(及蒙古)草原为主 \\
\hline & & $\mathrm{Dp}$ & 天山或包括附近山地 \\
\hline \multirow[t]{13}{*}{$\mathrm{P}$} & \multirow{13}{*}{$\begin{array}{l}\text { 高地型(以青藏高原为中心可包括其外围山 } \\
\text { 地) } \\
\text { Highland Type (Species whose distributon is } \\
\text { centered on the Qinghai-Tibet Plateau or } \\
\text { nearby) }\end{array}$} & $\mathrm{P}$ & $\begin{array}{l}\text { 高地型(以青藏高原为中心可包括 } \\
\text { 其外围山地) }\end{array}$ \\
\hline & & $\mathrm{Pa}$ & 包括附近山地 \\
\hline & & $\mathrm{Pb}$ & 㒸塘与大湖区 \\
\hline & & $\mathrm{Pc}$ & 青藏高原东部 \\
\hline & & $\mathrm{Pd}$ & 青藏高原东南部 \\
\hline & & $\mathrm{Pe}$ & 西部 \\
\hline & & $\mathrm{Pf}$ & 东北部 \\
\hline & & $\mathrm{Pg}$ & 北部 \\
\hline & & $\mathrm{Ph}$ & 南部 \\
\hline & & $\mathrm{Pw}$ & $\begin{array}{l}\text { 包括天山与横断山中部或更包括 } \\
\text { 附近山地 }\end{array}$ \\
\hline & & $\mathrm{Px}$ & 包括天山或再包括附近山地 \\
\hline & & Py & 主要包括横断山地 \\
\hline & & $\mathrm{Pz}$ & 包括横断山地中部并向东伸延 \\
\hline \multirow[t]{2}{*}{$\mathrm{H}$} & \multirow{2}{*}{$\begin{array}{l}\text { 喜马拉雅-横断山区型 Himalaya-Hengduan } \\
\text { Mountains Type }\end{array}$} & $\mathrm{H}$ & 喜马拉雅-横断山区型 \\
\hline & & $\mathrm{Ha}$ & 喜马拉雅南坡 \\
\hline
\end{tabular}


郜二虎, 何杰坤, 王志臣, 徐扬, 唐小平, 江海声. 全国陆生野生动物调查单元区划方案. 生物多样性, 2017, 25 (12): 1321-1330.

http://www.biodiversity-science.net/CN/10.17520/biods.2017135

\begin{tabular}{|c|c|c|c|}
\hline $\begin{array}{c}\text { 一级型代码 } \\
\text { Code }\end{array}$ & $\begin{array}{c}\text { 一级型 } \\
\text { Chorotype name }\end{array}$ & $\begin{array}{c}\text { 二级型代码 } \\
\text { Code }\end{array}$ & $\begin{array}{c}\text { 二级型 } \\
\text { Chorotype name }\end{array}$ \\
\hline & & $\mathrm{Hb}$ & 喜马拉雅及附近山地 \\
\hline & & $\mathrm{He}$ & $\begin{array}{l}\text { 喜马拉雅东南部(喜马拉雅-横断山 } \\
\text { 交汇地区) }\end{array}$ \\
\hline & & Hd & 雅鲁藏布江流域 \\
\hline & & $\mathrm{Hm}$ & 横断山及喜马拉雅(南翼为主) \\
\hline & & $\mathrm{Hc}$ & 横断山为主 \\
\hline \multirow[t]{5}{*}{ Y } & \multirow[t]{5}{*}{ 云贵高原 Yunnan-Guizhou Plateau Type } & Y & 云贵高原 \\
\hline & & Ya & 包括附近山地 \\
\hline & & $\mathrm{Yb}$ & 包括横断山南部 \\
\hline & & Yc & $a+b$ \\
\hline & & Yd & 大部分地区 \\
\hline \multirow[t]{13}{*}{$\mathrm{S}$} & \multirow[t]{13}{*}{ 南中国型 Southern China Type } & $\mathrm{S}$ & 南中国型 \\
\hline & & $\mathrm{Sa}$ & 热带 \\
\hline & & $\mathrm{Sb}$ & 热带-南亚热带 \\
\hline & & $\mathrm{Sc}$ & 热带-中亚热带 \\
\hline & & $\mathrm{Sd}$ & 热带-北亚热带 \\
\hline & & $\mathrm{Se}$ & 南亚热带-中亚热带 \\
\hline & & $\mathrm{Sf}$ & 南亚热带-北亚热带 \\
\hline & & $\mathrm{Sg}$ & 南亚热带 \\
\hline & & $\mathrm{Sh}$ & 中亚热带-北亚热带 \\
\hline & & $\mathrm{Si}$ & 中亚热带 \\
\hline & & Sn & 北亚热带 \\
\hline & & $\mathrm{Sm}$ & 热带-暖温带 \\
\hline & & $\mathrm{Sv}$ & 热带-中温带 \\
\hline \multirow[t]{8}{*}{ W } & \multirow{8}{*}{$\begin{array}{l}\text { 东洋型(包括少数旧热带型或环球热带-温带) } \\
\text { Oriental Type (including some Old Tropical } \\
\text { Type species and Globle Tropical Type } \\
\text { species) }\end{array}$} & $\mathrm{W}$ & $\begin{array}{l}\text { 东洋型(包括少数旧热带型或环球 } \\
\text { 热带-温带) }\end{array}$ \\
\hline & & Wa & 热带 \\
\hline & & $\mathrm{Wb}$ & 热带-南亚热带 \\
\hline & & Wc & 热带-中亚热带 \\
\hline & & $\mathrm{Wd}$ & 热带-北亚热带 \\
\hline & & We & 热带-温带 \\
\hline & & Wf & 中亚热带-北亚热带 \\
\hline & & Wo & 还分布于大洋洲热带 \\
\hline $\mathrm{J}$ & 岛屿型 Island Type & $\mathrm{J}$ & 岛屿型 \\
\hline $\mathrm{L}$ & 局地型 Local Type & $\mathrm{L}$ & 局地型 \\
\hline \multirow[t]{4}{*}{$\mathrm{O}$} & \multirow{4}{*}{$\begin{array}{l}\text { 不易归类(不易归类的分布, 其中不少分布 } \\
\text { 比较广泛的种, 大多与下列类型相似但又不 } \\
\text { 能视为其中的某一类) } \\
\text { Others (Including species whose distribution } \\
\text { is hard to classify. Some of them have a wide } \\
\text { distribution, with a chorotype similar to O1 to }\end{array}$} & $\mathrm{O}$ & $\begin{array}{l}\text { 不易归类的分布, 其中不少分布比 } \\
\text { 较广泛的种, 大多与下列类型相似 } \\
\text { 但又不能视为其中的某一类 }\end{array}$ \\
\hline & & $\mathrm{O} 1$ & 旧大陆温带、热带或温带-热带 \\
\hline & & $\mathrm{O} 2$ & 环球温带-热带 \\
\hline & & $\mathrm{O} 3$ & 地中海附近-中亚或包括东亚 \\
\hline
\end{tabular}


郜二虎, 何杰坤, 王志臣, 徐扬, 唐小平, 江海声. 全国陆生野生动物调查单元区划方案. 生物多样性, 2017, 25 (12): 1321-1330.

http://www.biodiversity-science.net/CN/10.17520/biods.2017135

\begin{tabular}{|c|l|l|l|}
\hline $\begin{array}{c}\text { 一级型代码 } \\
\text { Code }\end{array}$ & \multicolumn{1}{|c|}{$\begin{array}{c}\text { 一级型 } \\
\text { Chorotype name }\end{array}$} & \multicolumn{1}{c|}{$\begin{array}{c}\text { 二级型代码 } \\
\text { Code }\end{array}$} & \multicolumn{1}{c|}{$\begin{array}{c}\text { 二级型 } \\
\text { Chorotype name }\end{array}$} \\
\hline \multirow{2}{*}{} & \multirow{2}{*}{ O7) } & $\mathrm{O} 4$ & 旧大陆-北美 \\
\cline { 3 - 4 } & & $\mathrm{O} 5$ & 东半球(旧大陆-大洋洲)温带-热带 \\
\cline { 3 - 4 } & & O6 & 中亚-南亚或西南亚 \\
\cline { 3 - 4 } & & O7 & 亚洲中部 \\
\hline
\end{tabular}


郜二虎, 何杰坤, 王志臣, 徐扬, 唐小平, 江海声. 全国陆生野生动物调查单元区划方案. 生物多样性, 2017, 25 (12): 1321-1330,

http://www.biodiversity-science.net/CN/10.17520/biods.2017135

附录 7 环境指标及数据来源

Appendix 7 Environmental index and data resources

\begin{tabular}{|c|c|c|c|}
\hline 指标类型 & 精度 & 数据来源 & 指标 \\
\hline Index type & Accuracy & Data source & Index \\
\hline $\begin{array}{l}\text { 温度 } \\
\text { Temperature }\end{array}$ & $1 \mathrm{~km}$ & http://www.worldclim.org/ & $\begin{array}{l}\text { 年均气温、极端高温、极端低温、夏季均温、冬季均温、 } \geq 0^{\circ} \mathrm{C} \text { 积温 } \\
\text { Mean annual temperature, extremely high temperature, extremely low temperature, mean } \\
\text { summer temperature, mean winter temperature, } \geq 0^{\circ} \mathrm{C} \text { accumulative temperature }\end{array}$ \\
\hline $\begin{array}{l}\text { 降水 } \\
\text { Precipitation }\end{array}$ & $1 \mathrm{KM}$ & http://www.worldclim.org/ & $\begin{array}{l}\text { 年降水量、雨季降水量、旱季降水量、夏季降水量、冬季降水量 } \\
\text { Annual precipitation, precipitation in rain season, precipitation in dry season, precipitation in } \\
\text { summer, precipitation in winter }\end{array}$ \\
\hline $\begin{array}{l}\text { 地形 } \\
\text { Terrain }\end{array}$ & $90 \mathrm{~m}$ & $\begin{array}{l}\text { 国际农业研究磋商组织空间信息协会 } \\
\text { the Consortium for Spatial Information of the } \\
\text { Consultative Group for International Agricultural } \\
\text { Research }\end{array}$ & $\begin{array}{l}\text { 海拔、坡度 } \\
\text { Altitude, slope gradient }\end{array}$ \\
\hline $\begin{array}{l}\text { 地貌 } \\
\text { Landform }\end{array}$ & 1:400 万 & $\begin{array}{l}\text { 国家地球系统科学数据共享平台 } \\
\text { National Earth System Science Data Sharing } \\
\text { Infrastructure }\end{array}$ & $\begin{array}{l}\text { 地貌类型、海拔区间、地表起伏 } \\
\text { Geomorphological type, altitude range, surface relief }\end{array}$ \\
\hline $\begin{array}{l}\text { 植被 } \\
\text { Vegetation }\end{array}$ & 1:100 万 & $\begin{array}{l}\text { 《中华人民共和国植被图(1:100 万)》 } \\
\text { Vegetation map of the People’s Republic of China (1: } \\
1,000,000)\end{array}$ & $\begin{array}{l}\text { 植被类型 } \\
\text { Vegetation type }\end{array}$ \\
\hline $\begin{array}{l}\text { 土壤 } \\
\text { Soil }\end{array}$ & 1:100 万 & $\begin{array}{l}\text { 国家地球系统科学数据共享平台 } \\
\text { National Earth System Science Data Sharing } \\
\text { Infrastructure }\end{array}$ & $\begin{array}{l}\text { 土壤类型 } \\
\text { Soil type }\end{array}$ \\
\hline
\end{tabular}


郜二虎, 何杰坤, 王志臣, 徐扬, 唐小平, 江海声. 全国陆生野生动物调查单元区划方案. 生物多样性, 2017, 25 (12): 1321-1330.

http://www.biodiversity-science.net/CN/10.17520/biods.2017135

附录 8 全国陆生野生动物调查单元区划表

Appendix 8 Table of zoogeographical regions of China for terrestrial wildlife survey

\begin{tabular}{|c|c|c|c|c|c|}
\hline $\begin{array}{c}\text { 界 } \\
\text { Realm }\end{array}$ & $\begin{array}{c}\text { 区 } \\
\text { Region }\end{array}$ & $\begin{array}{c}\text { 亚区 } \\
\text { Subregion }\end{array}$ & $\begin{array}{c}\text { 地理省 } \\
\text { Geo-province }\end{array}$ & $\begin{array}{l}\text { 生态地理单元数量 } \\
\text { No. of } \\
\text { eco-geographical } \\
\text { units }\end{array}$ & $\begin{array}{c}\text { 调查单元数量 } \\
\text { No. of survey } \\
\text { units }\end{array}$ \\
\hline \multirow{6}{*}{$\begin{array}{l}\text { I 古北界 } \\
\text { Palaearctic realm }\end{array}$} & \multirow{5}{*}{$\begin{array}{l}\text { I1 东北区 } \\
\text { Northeast China region }\end{array}$} & \multirow{2}{*}{$\begin{array}{l}\text { I1A 大兴安岭亚区 } \\
\text { Daxing'anling Mountain subregion }\end{array}$} & $\begin{array}{l}\text { I1 Aa 大兴安岭北部省 } \\
\text { North Daxing'anling mountain province }\end{array}$ & 5 & 6 \\
\hline & & & $\begin{array}{l}\text { I1 Ab 大兴安岭南部省 } \\
\text { South Daxing'anling mountain province }\end{array}$ & 2 & 2 \\
\hline & & \multirow[t]{2}{*}{$\begin{array}{l}\text { I1B 长白山亚区 } \\
\text { Changbaishan Mountain subregion }\end{array}$} & $\begin{array}{l}\text { I1Bb 长白山地省 } \\
\text { Changbaishan mountain province }\end{array}$ & 7 & 10 \\
\hline & & & $\begin{array}{l}\text { I1Bc 三江平原省 } \\
\text { Sanjiang plain province }\end{array}$ & 1 & 1 \\
\hline & & $\begin{array}{l}\text { I1C 松辽平原亚区 } \\
\text { Song-liao Plain subregion }\end{array}$ & $\begin{array}{l}\text { I1Cc 辽河平原省 } \\
\text { Liaohe plain province }\end{array}$ & 3 & 4 \\
\hline & $\begin{array}{l}\text { I2 华北区 } \\
\text { North China region }\end{array}$ & $\begin{array}{l}\text { I2D 黄淮平原亚区 } \\
\text { Huang-huai Plain subregion }\end{array}$ & $\begin{array}{l}\text { I2Da 华北平原省 } \\
\text { North China plain province }\end{array}$ & 1 & 5 \\
\hline
\end{tabular}


郜二虎, 何杰坤, 王志臣, 徐扬, 唐小平, 江海声. 全国陆生野生动物调查单元区划方案. 生物多样性, 2017, 25 (12): 1321-1330.

$\mathrm{http}: / /$ www.biodiversity-science.net/CN/10.17520/biods.2017135

\begin{tabular}{|c|c|c|c|c|c|}
\hline $\begin{array}{c}\text { 界 } \\
\text { Realm }\end{array}$ & $\begin{array}{c}\text { 区 } \\
\text { Region }\end{array}$ & $\begin{array}{c}\text { 亚区 } \\
\text { Subregion }\end{array}$ & $\begin{array}{c}\text { 地理省 } \\
\text { Geo-province }\end{array}$ & $\begin{array}{l}\text { 生态地理单元数量 } \\
\text { No. of } \\
\text { eco-geographical } \\
\text { units }\end{array}$ & $\begin{array}{c}\text { 调查单元数量 } \\
\text { No. of survey } \\
\text { units }\end{array}$ \\
\hline & & & $\begin{array}{l}\text { I2Db 山东丘陵省 } \\
\text { Shangdong hills province }\end{array}$ & 2 & 2 \\
\hline & & & $\begin{array}{l}\text { I2Dc 淮北平原省 } \\
\text { Huaibei plain province }\end{array}$ & 1 & 4 \\
\hline & & \multirow{2}{*}{$\begin{array}{l}\text { I2E 黄土高原亚区 } \\
\text { Loess Plateau subregion }\end{array}$} & $\begin{array}{l}\text { I2Eb 晋南-渭河-伏牛省 } \\
\text { Jinnan-Weihe-Funiu province }\end{array}$ & 10 & 11 \\
\hline & & & $\begin{array}{l}\text { I2Ec 甘南-六盘省 } \\
\text { Gannan-Liupan province }\end{array}$ & 4 & 5 \\
\hline & \multirow{3}{*}{$\begin{array}{l}\text { I3 蒙新区 } \\
\text { Neimenggu-Xinjiang region }\end{array}$} & \multirow{3}{*}{$\begin{array}{l}\text { I3G 西部荒漠亚区 } \\
\text { West Desert subregion }\end{array}$} & $\begin{array}{l}\text { I3Ga 河套-河西省 } \\
\text { Hetao-Hexi province }\end{array}$ & 5 & 7 \\
\hline & & & $\begin{array}{l}\text { I3Gb 阿拉善-北山省 } \\
\text { Alashan-Beishan province }\end{array}$ & 2 & 2 \\
\hline & & & $\begin{array}{l}\text { I3Gc 东疆戈壁省 } \\
\text { East Xinjiang gobi province }\end{array}$ & 4 & 4 \\
\hline
\end{tabular}


郜二虎, 何杰坤, 王志臣, 徐扬, 唐小平, 江海声. 全国陆生野生动物调查单元区划方案. 生物多样性, 2017, 25 (12): 1321-1330.

$\mathrm{http}: / /$ www.biodiversity-science.net/CN/10.17520/biods.2017135

\begin{tabular}{|c|c|c|c|c|c|}
\hline $\begin{array}{c}\text { 界 } \\
\text { Realm }\end{array}$ & $\begin{array}{c}\text { 区 } \\
\text { Region }\end{array}$ & $\begin{array}{c}\text { 亚区 } \\
\text { Subregion }\end{array}$ & $\begin{array}{c}\text { 地理省 } \\
\text { Geo-province }\end{array}$ & $\begin{array}{c}\text { 生态地理单元数量 } \\
\text { No. of } \\
\text { eco-geographical } \\
\text { units }\end{array}$ & $\begin{array}{c}\text { 调查单元数量 } \\
\text { No. of survey } \\
\text { units }\end{array}$ \\
\hline & & & $\begin{array}{l}\text { I3Gd 准噶尔盆地省 } \\
\text { Junggar basin province }\end{array}$ & 4 & 4 \\
\hline & & & $\begin{array}{l}\text { I3Ge 塔里木盆地省 } \\
\text { Tarim basin province }\end{array}$ & 3 & 3 \\
\hline & & \multirow[b]{2}{*}{$\begin{array}{l}\text { I3H 天山山地亚区 } \\
\text { Tianshan Mountain subregion }\end{array}$} & $\begin{array}{l}\text { I3Ha 天山山地省 } \\
\text { Tianshan mountain province }\end{array}$ & 3 & 3 \\
\hline & & & $\begin{array}{l}\mathrm{I} 3 \mathrm{Hb} \text { 阿尔泰山地省 } \\
\text { Altai mountain province }\end{array}$ & 1 & 1 \\
\hline & \multirow{3}{*}{$\begin{array}{l}\text { I4 青藏区 } \\
\text { Qinghai-Xizang region }\end{array}$} & \multirow{3}{*}{$\begin{array}{l}\text { I4I 美塘高原亚区 } \\
\text { Qiang-Tang Plateau subregion }\end{array}$} & $\begin{array}{l}\text { I4Ib 昆仑省 } \\
\text { Kunlun province }\end{array}$ & 4 & 6 \\
\hline & & & $\begin{array}{l}\text { I4Ic 高原湖盆山地省 } \\
\text { Plateau lake basin province }\end{array}$ & 4 & 4 \\
\hline & & & $\begin{array}{l}\text { I4Id 帕米尔高原省 } \\
\text { Pamir plateau province }\end{array}$ & 1 & 1 \\
\hline
\end{tabular}


郜二虎, 何杰坤, 王志臣, 徐扬, 唐小平, 江海声. 全国陆生野生动物调查单元区划方案. 生物多样性, 2017, 25 (12): 1321-1330.

$\mathrm{http}: / /$ www.biodiversity-science.net/CN/10.17520/biods.2017135

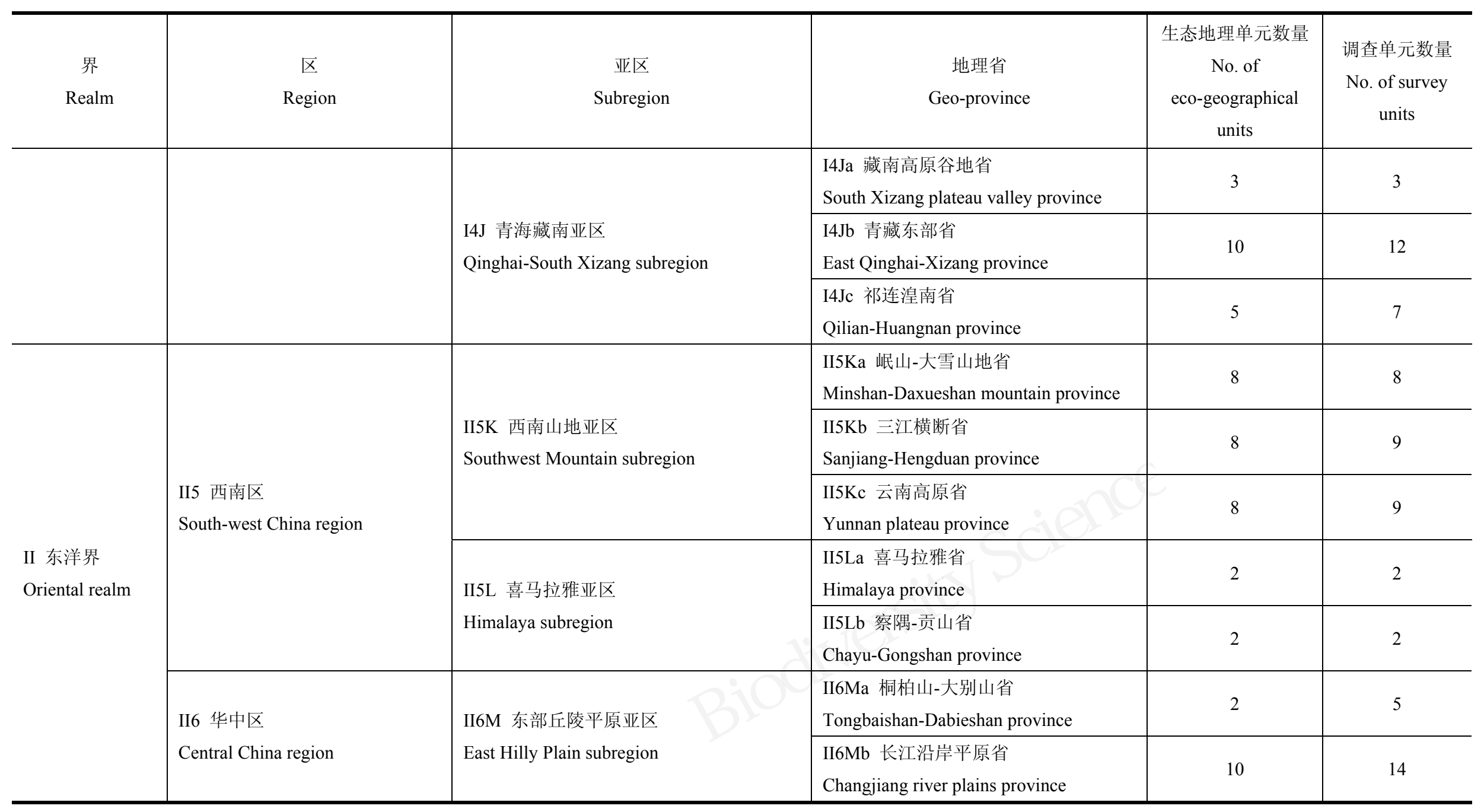


郜二虎, 何杰坤, 王志臣, 徐扬, 唐小平, 江海声. 全国陆生野生动物调查单元区划方案. 生物多样性, 2017, 25 (12): 1321-1330.

$\mathrm{http}: / /$ www.biodiversity-science.net/CN/10.17520/biods.2017135

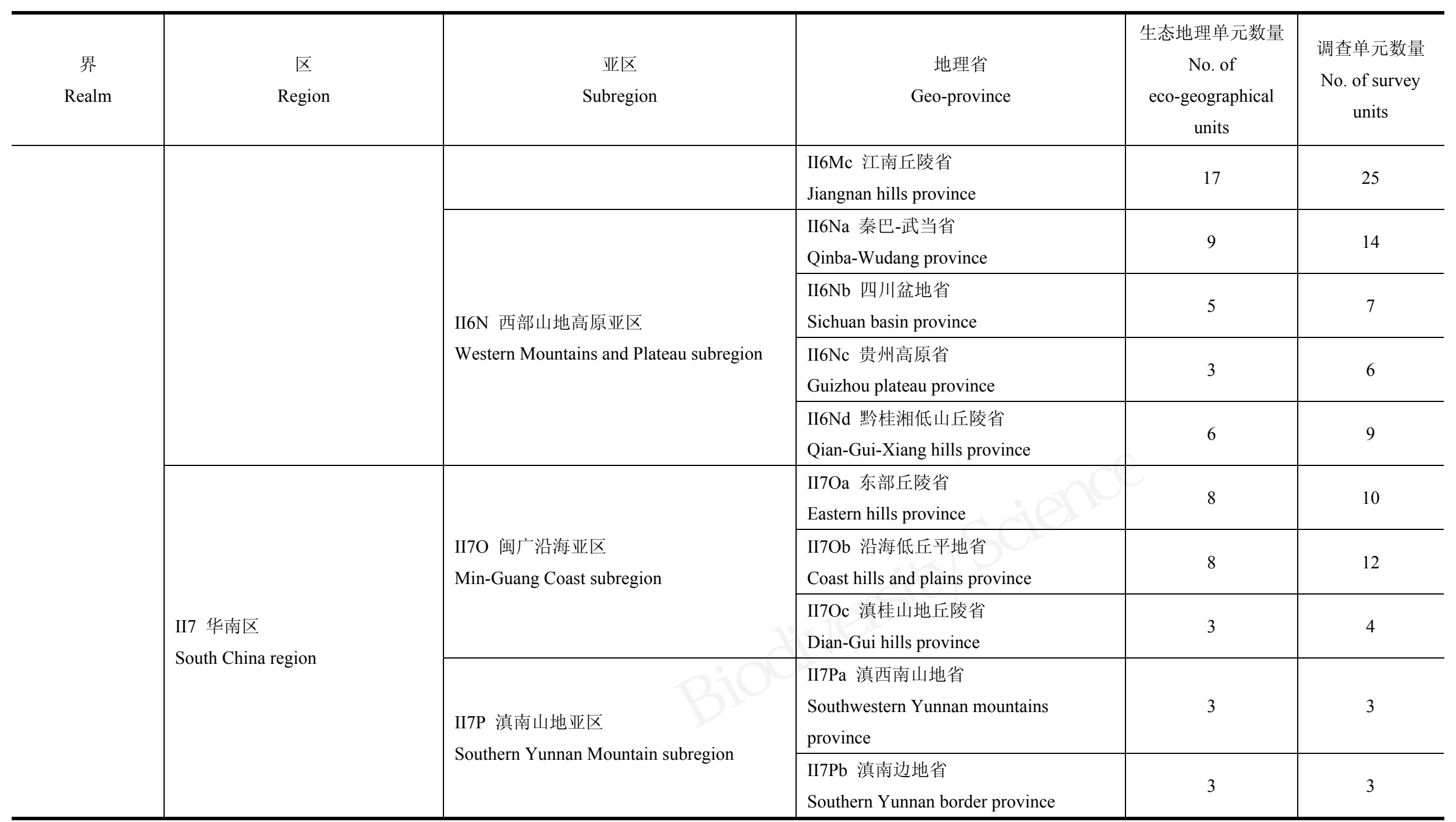


郜二虎, 何杰坤, 王志臣, 徐扬, 唐小平, 江海声. 全国陆生野生动物调查单元区划方案. 生物多样性, 2017, 25 (12): 1321-1330.

$\mathrm{http}: / /$ www.biodiversity-science.net/CN/10.17520/biods.2017135

\begin{tabular}{|c|c|c|c|c|c|}
\hline $\begin{array}{c}\text { 界 } \\
\text { Realm }\end{array}$ & $\begin{array}{c}\text { 区 } \\
\text { Region }\end{array}$ & $\begin{array}{c}\text { 亚区 } \\
\text { Subregion }\end{array}$ & $\begin{array}{c}\text { 地理省 } \\
\text { Geo-province }\end{array}$ & $\begin{array}{c}\text { 生态地理单元数量 } \\
\text { No. of } \\
\text { eco-geographical } \\
\text { units }\end{array}$ & $\begin{array}{c}\text { 调查单元数量 } \\
\text { No. of survey } \\
\text { units }\end{array}$ \\
\hline & & \multirow{2}{*}{$\begin{array}{l}\text { II7Q 海南亚区 } \\
\text { Hainan subregion }\end{array}$} & $\begin{array}{l}\text { II7Qa 中部山地省 } \\
\text { Central mountains province }\end{array}$ & 1 & 1 \\
\hline & & & $\begin{array}{l}\text { II7Qb 沿海低地省 } \\
\text { Coast lowland province }\end{array}$ & 2 & 2 \\
\hline & & $\begin{array}{l}\text { II7R 台湾亚区 } \\
\text { Taiwan subregion }\end{array}$ & $\begin{array}{l}\text { II7Rb 西部低地省 } \\
\text { Western lowland province }\end{array}$ & 4 & 4 \\
\hline & & $\begin{array}{l}\text { II7S 南海诸岛亚区 } \\
\text { South China Sea Islands subregion }\end{array}$ & $\begin{array}{l}\text { II7Sa 南海诸岛省 } \\
\text { South China Sea islands province }\end{array}$ & 1 & 2 \\
\hline
\end{tabular}

